Preprared in cooperation with the National Park Service, Voyageurs National Park, Minnesota

\title{
Impact of Wildfire on Levels of Mercury in Forested Watershed Systems_-Voyageurs National Park, Minnesota
}

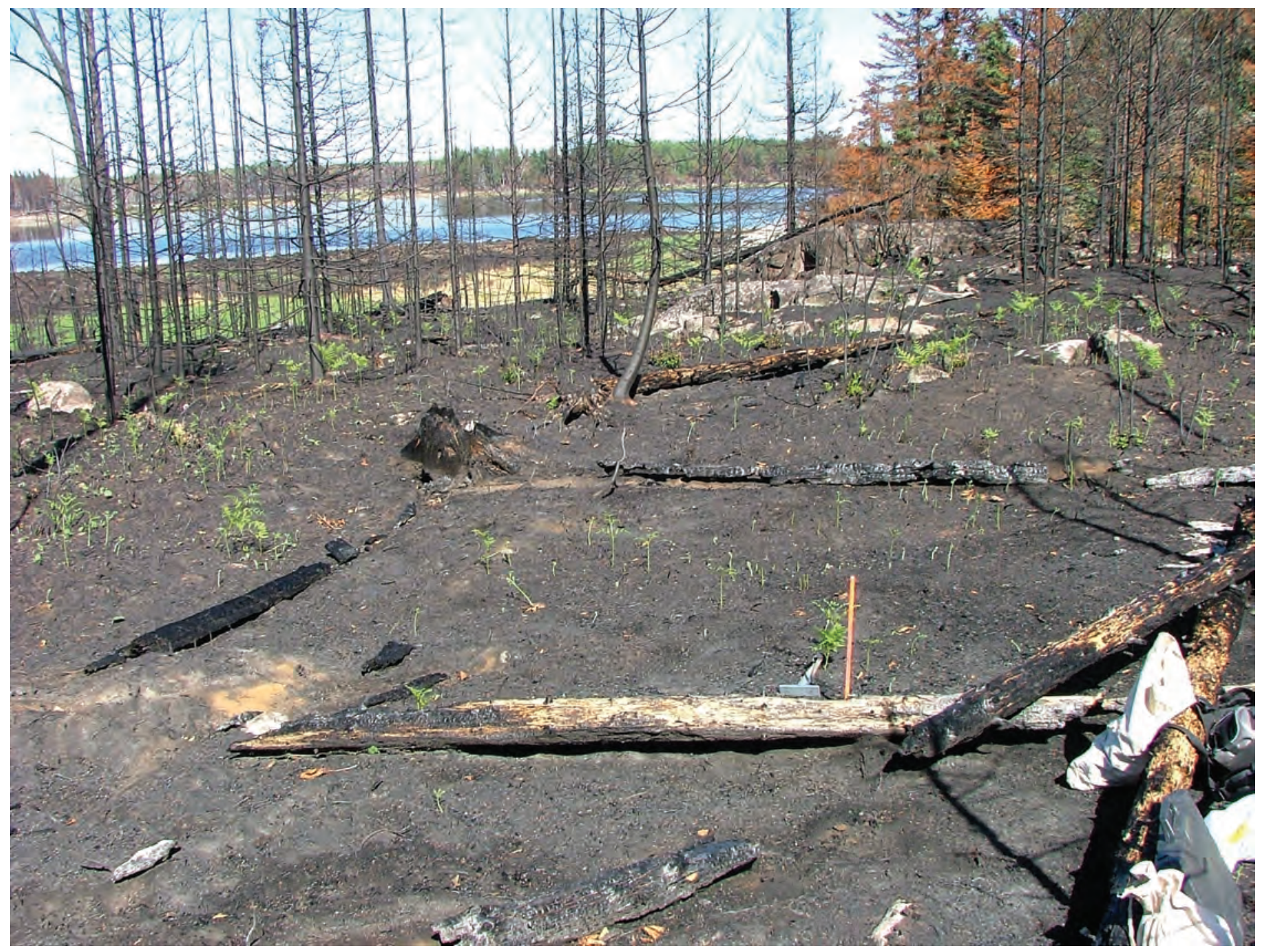

Scientific Investigations Report 2009-5151 


\section{Impact of Wildfire on Levels of Mercury in Forested Watershed Systems- Voyageurs National Park, Minnesota}

By Laurel G. Woodruff, Mark B. Sandheinrich, Mark E. Brigham, and William F. Cannon

Prepared in cooperation with the National Park Service, Voyageurs National Park, Minnesota

Scientific Investigations Report 2009-5151 


\title{
U.S. Department of the Interior \\ KEN SALAZAR, Secretary
}

\author{
U.S. Geological Survey \\ Suzette M. Kimball, Acting Director
}

U.S. Geological Survey, Reston, Virginia: 2009

For more information on the USGS - the Federal source for science about the Earth, its natural and living resources, natural hazards, and the environment, visit http://www.usgs.gov or call 1-888-ASK-USGS

For an overview of USGS information products, including maps, imagery, and publications, visit http://www.usgs.gov/pubprod

To order this and other USGS information products, visit http://store.usgs.gov

Any use of trade, product, or firm names is for descriptive purposes only and does not imply endorsement by the U.S. Government.

Although this report is in the public domain, permission must be secured from the individual copyright owners to reproduce any copyrighted materials contained within this report.

On the cover. Photograph of soil sample site along the shoreline of Shoepack Lake; sampling was done in August 2004, following a wildfire in Section 33, Voyageurs National Park, Minnesota.

Suggested citation:

Woodruff, L.G., Sandheinrich, M.B., Brigham, M.E., and Cannon, W.F., Impact of wildfire on levels of mercury in forested watershed systems — Voyageurs National Park, Minnesota: U.S. Geological Survey, Scientific Investigations Report 2009-5151, 19 p. plus appendices, available online only at http://pubs.usgs.gov/sir/2009/5151/. 


\section{Preface}

Mercury $(\mathrm{Hg})$ cycling in forest soils is strongly influenced by forest disturbance, especially forest fires. Soil, lake water, and age-1 yellow perch were collected from lakes and surrounding watersheds in Voyageurs National Park, MN, to study the effect of fire on mercury. Samples were collected within the footprint of the Section 33 wildfire, which burned over $4.047 \mathrm{~km}^{2}$ in the Shoepack Lake watershed in 2004, and from lakes and catchments in nearby unburned areas of the Park. Analyses of O- and A-horizon soils from the Shoepack Lake fire area show losses relative to adjacent unburned areas of (1) $\sim 0.22 \mathrm{mg} \mathrm{Hg} / \mathrm{m}^{2}$ in high-fire severity burned areas because of complete combustion of the $\mathrm{O}$ horizon, and (2) $\sim 0.6$ to $0.9 \mathrm{mg} \mathrm{Hg} / \mathrm{m}^{2}$ from the A horizon in these same areas. The soil survey was conducted 2 weeks after the fire and prior to any significant rainfall and indicates that these mercury losses were an immediate and direct consequence of combustion and volatilization of mercury from the soil. Soil samples from the same sites collected in 2005 and 2006 showed no significant changes in mercury concentrations in either O- or A-horizon soils. Water-column data were collected three times a year (spring, mid-summer, and late summer) from 2000 through 2006 from four Voyageurs lakes, including Shoepack Lake and three nonburn control lakes. Over this short time period, there were significant $(p<0.05$, where $p=$ probability) decreases in aqueous methylmercury concentrations in three of the four lakes, but among the lakes there were no discernible patterns in lake-water chemistry that could be attributed to burned watershed vs. unburned watershed factors. There was not a significant difference in concentrations of total mercury in yellow perch from Shoepack Lake in 2005 and 2006 (post-fire) relative to that measured during 2000 and 2001 (pre-fire). There also was no detectable difference in the growth of age-1 yellow perch in Shoepack Lake between 2005 and 2006. The lack of change in methylmercury concentrations in yellow perch from Shoepack Lake before and after the fire indicates a lack of effect of fire on the bioavailability of methylmercury in the lake. There also was no apparent increase in nutrients following the fire that would have affected perch growth rate. Taken together, data for soil, lake water, and age-1 yellow perch show that mobilization of a significant quantity of mercury from soils resulting from the Section 33 wildfire had no effect on mercury levels in either lake waters or age-1 yellow perch in adjacent Shoepack Lake. 


\section{Acknowledgments}

This study was conducted in cooperation with the National Park Service (NPS) in Voyageurs National Park, MN. Funding was provided by the National Park Service, the U.S. Geological Survey (USGS) Mineral Resources Program, the USGS Minnesota Water Science Center, and the University of Wisconsin-La Crosse Wisconsin (UW-LX). Cooperation and support from Jim Schaberl, Chris Holbeck, and Steve Windels (NPS) is greatly appreciated. Field support for soil sampling was provided by Connie Dicken and John Horton (USGS). Sean Bailey, Matthew Brantner, Leland Grimm (NPS), Peter Liska, Kevin Miller, and Jake Ogorek (UW-LX) assisted in the field during collection of age-1 yellow perch. Sean Bailey, Rebecca Curland, and Ryan Legler (UW-LX) assisted in the laboratory analysis of mercury in the yellow perch. Greg Melhus, Wally Larson, and Larry Kallemeyn (USGS), and Joan Elias (NPS) assisted with water sampling. 


\section{Contents}

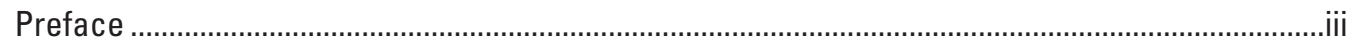

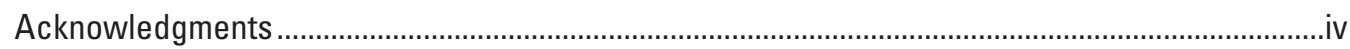

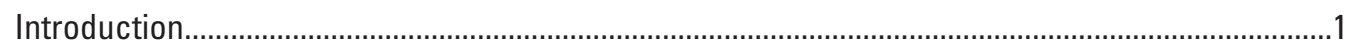

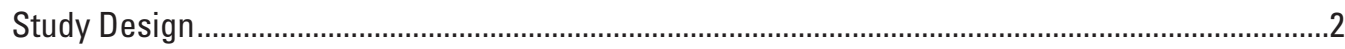

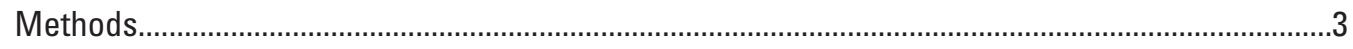

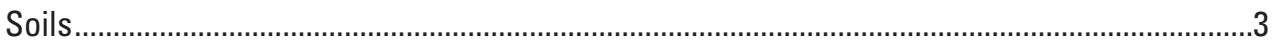

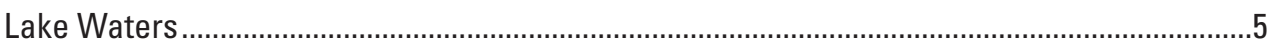

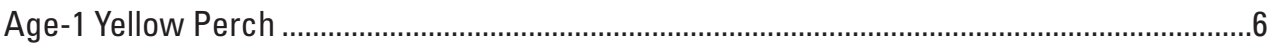

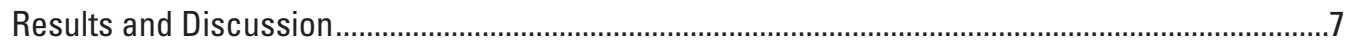

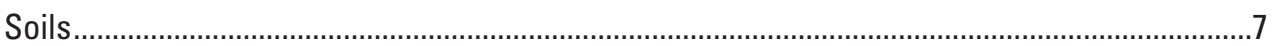

Preburn Soil Chemistry ................................................................................................

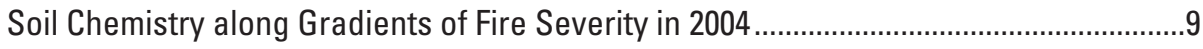

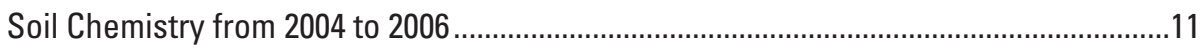

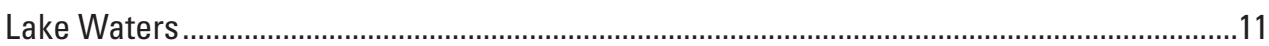

Shoepack Lake Waters_Comparison of Pre-Fire and Post-Fire Conditions..................12

Shoepack Lake Waters Compared to Control Lakes .......................................................12

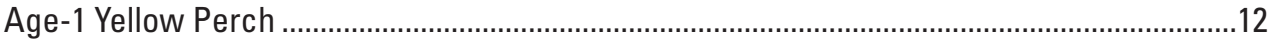

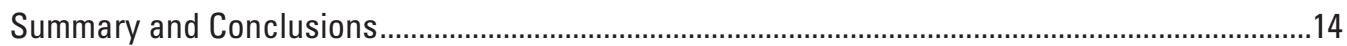

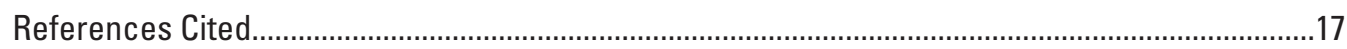

Appendixes 1, 2, 3, 4, 5, 6, 7, 8, and 9.......................................................................................

(Click on appendix number above to access appendix.)

\section{Figures}

1. Location map of Voyageurs National Park, Minnesota.....

2. Location map showing perimeter of Section 33 wildfire footprint (August 11, 2004) and 10 soil sample sites

3. Photographs of soil sample areas and collection sites characterized by fire severity

4. Soil pit from the Ryan Lake watershed in Voyageurs National Park showing different soil horizons.

5. Box plots of mercury and carbon concentrations in A- and B-horizon soils from Brown, Ryan, and Shoepack Lakes watersheds.

6. Box plots of concentrations and calculated areal burden for mercury in 0-, $A-$, and B-horizon soils from unburned sites within the Section 33 wildfire footprint in the Shoepack Lake watershed

7. Box plots of calculated areal burdens for carbon and mercury in 0- and A-horizon soils from sample sites within the Section 33 fire footprint in the Shoepack Lake watershed collected across gradients of fire severity.

8. Plot of mercury versus carbon for A-horizon soils across fire-severity gradients in Section 33 wildfire footprint in Shoepack Lake watershed.

9. Photographs showing vegetation regeneration from high-severity soil site 6-3 following the Section 33 wildfire 
10. Box plots of calculated loads for carbon and mercury in 0- and A-horizon soils from sample sites within the Section 33 wildfire footprint in the Shoepack Lake watershed.

11. Plot illustrating data and least square regession trendlines in methylmercury concentrations in unfiltered lake waters from the four study lakes 14

12. Box plots of total mercury concentrations in age-1 yellow perch

\section{Tables}

1. Timetable for collection of various sample media in Voyageurs National Park, Minn.

2. Summary results of quality-assurance analyses during determination of total mercury in samples of yellow perch sampled in 2003-2006 from Brown, Peary, Ryan, and Shoepack Lakes in Voyageurs National Park, Minn.

3. Analytical results for total mercury in reference materials from the U.S. National Institute of Standards and Technology and the National Research Council of Canada.

4. Mercury concentrations in A-horizon soils at 10 sites sampled in 2004 within Section 33 wildfire areas. Soils were collected across gradients of fire severity

5. Median areal burdens for mercury and carbon in 0-horizon and A-horizon soils collected in 2004 within Section 33 wildfire areas

6. Characteristics of age-1 yellow perch sampled from Brown, Peary, Ryan, and Shoepack Lakes in Voyageurs National Park in May 2003-2006.

7. Analysis of variance used to test for differences in mercury concentrations and total length of age-1 yellow perch for the three control lakes and Shoepack Lake before and after the Section 33 wildfire of 2004 


\section{Conversion Factors and Datums}

\begin{tabular}{|c|c|c|}
\hline Multiply & By & To obtain \\
\hline \multicolumn{3}{|c|}{ Length } \\
\hline centimeter $(\mathrm{cm})$ & 0.3937 & inch (in.) \\
\hline millimeter (mm) & 0.03937 & inch (in.) \\
\hline meter $(\mathrm{m})$ & 3.281 & foot (ft) \\
\hline meter $(\mathrm{m})$ & 1.094 & yard (yd) \\
\hline \multicolumn{3}{|c|}{ Area } \\
\hline square meter $\left(\mathrm{m}^{2}\right)$ & 0.0002471 & acre \\
\hline square kilometer $\left(\mathrm{km}^{2}\right)$ & 247.1 & acre \\
\hline square meter $\left(\mathrm{m}^{2}\right)$ & 10.76 & square foot $\left(\mathrm{ft}^{2}\right)$ \\
\hline \multicolumn{3}{|c|}{ Volume } \\
\hline liter $(\mathrm{L})$ & 33.82 & ounce, fluid (fl. oz) \\
\hline liter (L) & 2.113 & pint $(p t)$ \\
\hline liter $(\mathrm{L})$ & 1.057 & quart (qt) \\
\hline liter $(\mathrm{L})$ & 0.2642 & gallon (gal) \\
\hline cubic centimeter $\left(\mathrm{cm}^{3}\right)$ & 0.06102 & cubic inch $\left(\mathrm{in}^{3}\right)$ \\
\hline liter $(\mathrm{L})$ & 61.02 & cubic inch $\left(\mathrm{in}^{3}\right)$ \\
\hline \multicolumn{3}{|c|}{ Mass } \\
\hline $\operatorname{gram}(\mathrm{g})$ & 0.03527 & ounce, avoirdupois (oz) \\
\hline kilogram (kg) & 2.205 & pound avoirdupois (lb) \\
\hline nanogram (ng) & 0.0000000000353 & ounce, avoirdupois (oz) \\
\hline \multicolumn{3}{|c|}{ Density } \\
\hline kilogram per cubic meter $\left(\mathrm{kg} / \mathrm{m}^{3}\right)$ & 0.06242 & pound per cubic foot $\left(\mathrm{lb} / \mathrm{ft}^{3}\right)$ \\
\hline
\end{tabular}

Temperature in degrees Celsius $\left({ }^{\circ} \mathrm{C}\right)$ may be converted to degrees Fahrenheit $\left({ }^{\circ} \mathrm{F}\right)$ as follows:

${ }^{\circ} \mathrm{F}=\left(1.8 x^{\circ} \mathrm{C}\right)+32$

Temperature in degrees Fahrenheit $\left({ }^{\circ} \mathrm{F}\right)$ may be converted to degrees Celsius $\left({ }^{\circ} \mathrm{C}\right)$ as follows:

${ }^{\circ} \mathrm{C}=\left({ }^{\circ} \mathrm{F}-32\right) / 1.8$

Concentrations of chemical constituents in soils are given either in parts per million (ppm) or weight percent (wt. \%). Calculated areal burdens are given as milligrams per meter squared $(\mathrm{mg} / \mathrm{m} 2)$ or grams per meter squared $(\mathrm{g} / \mathrm{m} 2)$. Concentrations of chemical constituents in water are given in milligrams per liter (mg/L), micrograms per liter $(\mu \mathrm{g} / \mathrm{L})$, or nanograms per liter $(\mathrm{ng} / \mathrm{L})$. Concentrations of mercury in age-one yellow perch are given in nanograms per gram $(\mathrm{ng} / \mathrm{g})$. 



\title{
Impact of Wildfire on Levels of Mercury in Forested Watershed Systems-Voyageurs National Park, Minnesota
}

\author{
By Laurel G. Woodruff, ${ }^{1}$ Mark B. Sandheinrich, ${ }^{2}$ Mark E. Brigham, ${ }^{1}$ and William F. Cannon ${ }^{1}$
}

\section{Introduction}

Atmospheric deposition of mercury to remote lakes in mid-continental and eastern North America has increased approximately threefold since the mid-1800s (Swain and others, 1992; Fitzgerald and others, 1998; Engstrom and others,

${ }^{1}$ U.S. Geological Survey.

${ }^{2}$ University of Wisconsin-LaCrosse.
2007). As a result, concerns for human and wildlife health related to mercury contamination have become widespread. Despite an apparent recent decline in atmospheric deposition of mercury in many areas of the Upper Midwest (Engstrom and Swain, 1997; Engstrom and others, 2007), lakes in which fish contain levels of mercury deemed unacceptable for human consumption and possibly unacceptable for fish-consuming wildlife are being detected with increasing frequency. In northern Minnesota, Voyageurs National Park (VNP) (fig. 1) protects a series of southern boreal lakes and wetlands situated on bedrock of the Precambrian Canadian Shield. Mercury

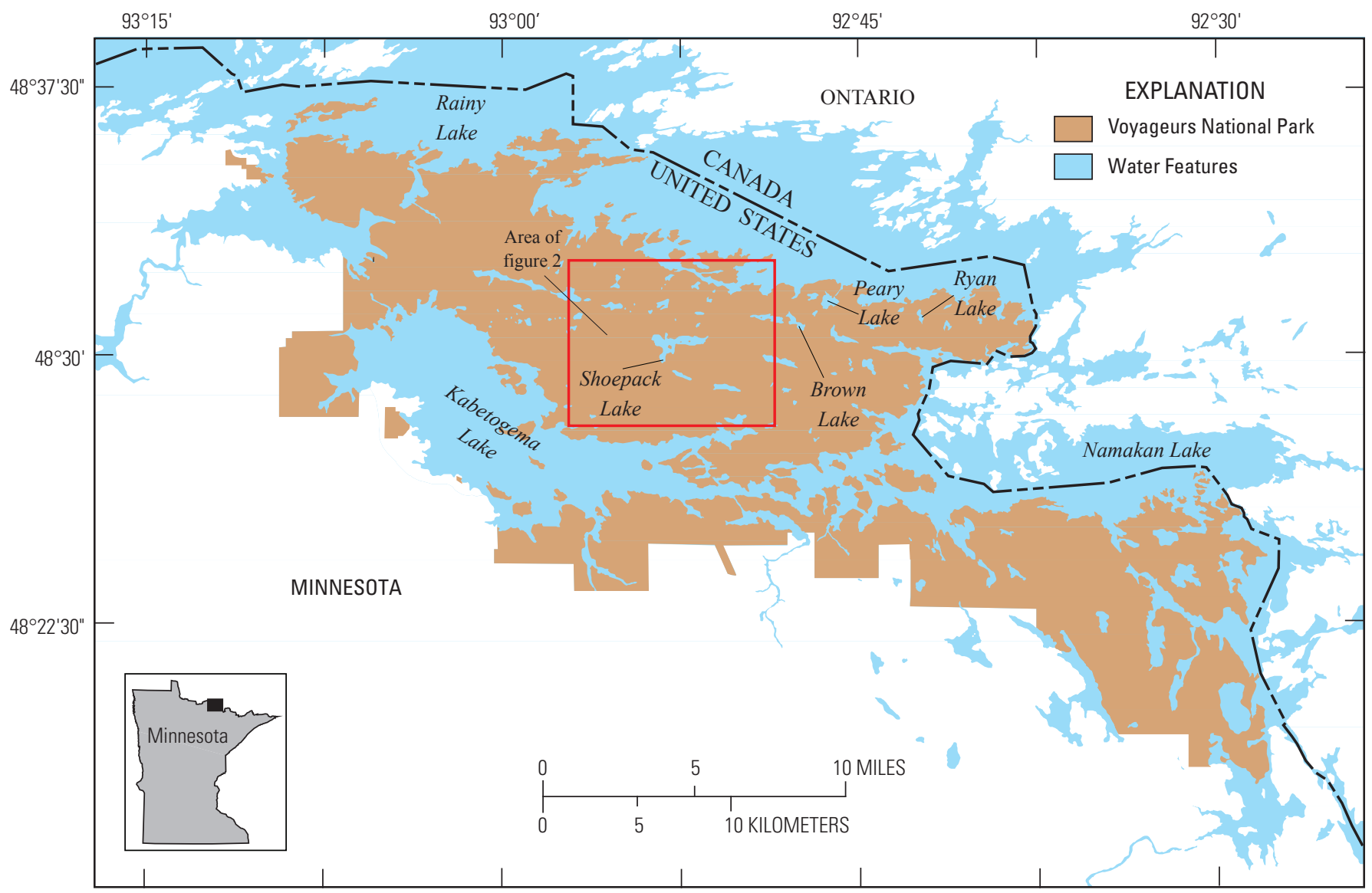

Figure 1. Location map of Voyageurs National Park, Minnesota. 
contamination has become a significant resource issue within VNP as high concentrations of mercury in loons, bald eagle eaglets, grebes, northern pike, and other species of wildlife and fish have been found. The two most mercury-contaminated lakes in Minnesota, measured as methylmercury in northern pike (Esox lucius), are in VNP.

Recent multidisciplinary U.S. Geological Survey (USGS) research demonstrated that the bulk of the mercury in lake waters, soils, and fish in VNP results from atmospheric deposition (Wiener and others, 2006). The study by Wiener and others (2006) showed that the spatial distribution of mercury in watershed soils, lake waters, and age-1 yellow perch (Perca flavescens) within the Park was highly variable. The majority of factors correlated for this earlier study suggested that mercury concentrations in lake waters and age- 1 yellow perch reflected the influence of ecosystem processes that affected within-lake microbial production and abundance of methylmercury (Wiener and others, 2006), while the distribution of mercury in watershed soils seemed to be partially dependent on forest disturbance, especially the historic forest fire pattern (Woodruff and Cannon, 2002).

Forest fire has an essential role in the forest ecosystems of VNP (Heinselman, 1996). Because resource and land managers need to integrate both natural wildfire and prescribed fire in management plans, the potential influence of fire on an element as sensitive to the environment as mercury becomes a critical part of their decisionmaking. A number of recent studies have shown that while fire does have a significant impact on mercury at the landscape level, the observed effects of fire on aquatic environments are highly variable and unpredictable (Caldwell and others, 2000; Garcia and Carrigan, 2000; Kelly and others, 2006; Nelson and others, 2007). Caldwell and others (2000) described an increase in methylmercury in reservoir sediments resulting from mobilization and transport of charred vegetative matter following a fire in New Mexico. Krabbenhoft and Fink (2000) attributed increases in total mercury concentrations in young-of-the-year fish in the Florida Everglades to release of mercury resulting from peat oxidation following fires. A fivefold increase in whole-body mercury accumulation by rainbow trout (Oncorhynchus mykiss) following a fire in Alberta, Canada, apparently resulted from increased nutrient concentrations that enhanced productivity and restructured the food web of a lake within the fire's burn footprint (Kelly and others, 2006).

For this study, we determined the short-term effects of forest fire on mercury concentrations in terrestrial and aquatic environments in VNP by comparing and contrasting mercury concentrations in forest soils, lake waters, and age-1 yellow perch for a burned watershed and an adjacent lake, with similar samples from watersheds and lakes with no fire activity (control watersheds and lakes). The concentration of total mercury in whole, 1-year-old yellow perch serves as a good biological indicator for monitoring trends in methylmercury concentrations in food webs of lakes in North America (Wiener and others, 2007). With a limited gape, age-1 yellow perch that hatched the previous year and resided in a lake for 1 year feed largely on zooplankton and small benthic invertebrates. Thus, age-1 yellow perch provide a baseline for methylmercury concentrations for individual lakes that can be compared across spatial areas.

The nine appendixes that accompany this report contain the complete datasets for soils, lake waters, and age-1 yellow perch collected for this study. This report uses data from these three media to provide a framework for evaluating short-term effects of fire on mercury in forested soils and possible effects of the mobilization of mercury from soils on lake water quality and aquatic health.

\section{Study Design}

From 2004 to 2006, the USGS and University of Wisconsin-LaCrosse (UW-LX), in cooperation with the National Park Service (NPS), collected soils, lake waters, and age-1 yellow perch from Shoepack Lake and its adjacent watershed (fig. 1), within an area that burned in a natural wildfire in August 2004. The objectives of this study were to determine the effects of the 2004 wildfire on mercury concentrations in burned watershed soils, in lake waters, and in age- 1 yellow perch in Shoepack Lake. These same three sets of sample media were also collected from lakes and watersheds far removed from the 2004 fire area (Peary Lake, Brown Lake, and Ryan Lake) (fig. 1) to act as unburned controls.

An initial study requested by the NPS was designed around a proposed 2004 prescribed fire scheduled for an area that encompassed much of the Ryan Lake watershed in VNP. The unburned control lakes and watersheds for the original study design were nearby Brown Lake and Peary Lake, located west of the proposed prescribed fire area. In 2003, lake waters and age-1 yellow perch were collected from these three lakes as preburn baseline samples. Soils were collected only from the Brown Lake and Ryan Lake watersheds.

An indefinite postponement of the Ryan Lake prescribed fire and the outbreak of a lightning-ignited wildfire in July 2004 in the interior of the Kabetogema Peninsula, a remote part of VNP, shifted the geographic focus of the study. By August 2004, when the wildfire (by then known as the Section 33 fire) was extinguished by rain, it had burned more than $4.047 \mathrm{~km}^{2}$, including nearly $80 \%$ of the shoreline of Shoepack Lake (fig. 2). The shift of this research from a study designed around prescribed fire to a study centered on a wildfire provided an opportunity to examine intensified fire effects. The timing of prescribed fire is largely controlled by soil-moisture levels and weather conditions, in part to ensure that fire severity, defined as the impact of the heat pulse to the basal surface, is generally low. In contrast, wildfires usually take off under uncontrolled conditions of abundant, dry fuel loads and windy, dry weather, with resulting high variability in fire severity influenced by local conditions.

Lake waters and age- 1 yellow perch had been collected in the 2004 field season from Ryan Lake, Brown Lake, and Peary 


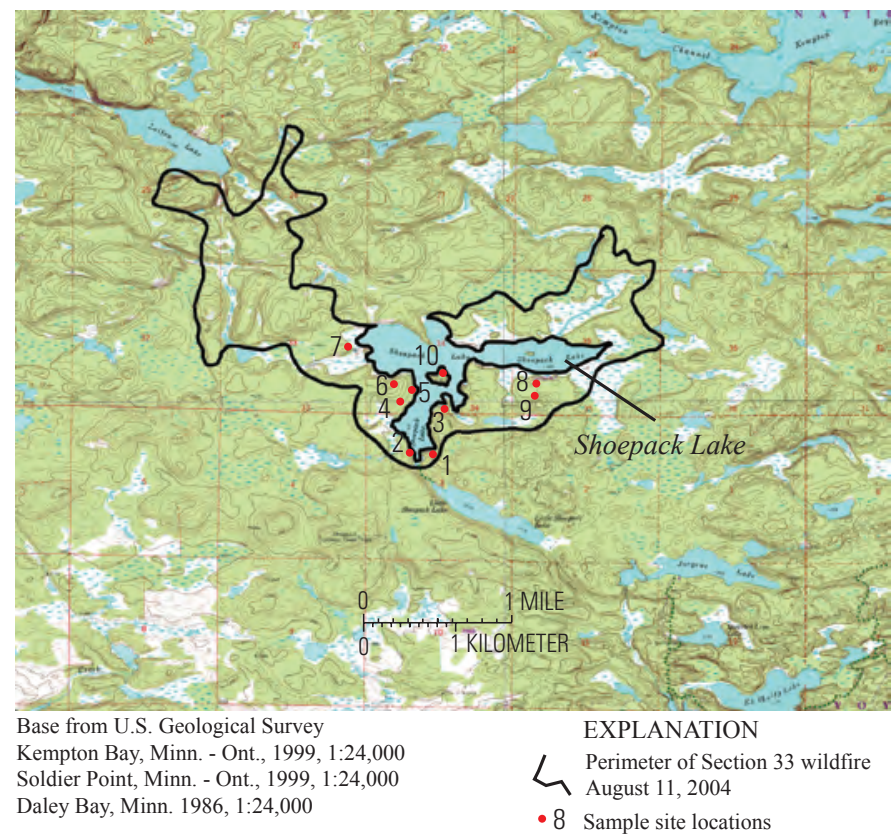

Figure 2. Location map showing perimeter of Section 33 wildfire footprint (August 11, 2004) and 10 soil sample sites. (See table 4.)

Lake. After the Section 33 fire, lake waters from Shoepack Lake were collected in August and September 2004. Soils were collected from the Shoepack Lake watershed in August 2004, 2 weeks after the Section 33 fire had burned out. In 2005 and 2006, lake waters and age-1 yellow perch were collected from Shoepack Lake, Ryan Lake, Brown Lake, and Peary Lake. Soils were collected from the Shoepack Lake watershed in 2005 and 2006 and from the Ryan Lake and Brown Lake watersheds in 2006. In all cases, soils were collected in close proximity, usually within 1 to 2 meters, to the original sample sites that had been marked with metal stakes in the first year of collecting. For clarity, the complete sampling schedule for all three media described above is summarized in table 1.

\section{Methods}

\section{Soils}

To obtain equivalents to preburn "control" sites within the Section 33 wildfire footprint, the irregular burn pattern of the fire was exploited. Within the footprint of the fire, soil samples were collected along gradients of fire severity, here defined as the effect of the fire to the forest floor. Ten sampling areas were selected near the shoreline of Shoepack Lake in burned areas (fig. 2). Within each area, three sites that had similar topography and vegetation but varying fire severity (unburned, low-moderate severity, and high severity) were selected. Criteria defining low-moderate and high-severity sites were based on field observations of the extent of combustion of forest-floor organic soils. Photographs of site 4 in figure 3 are examples of the visual and physical criteria used in the field for site-severity classification. Unburned sites were largely untouched by fire, although in some cases the forest canopy nearby was scorched (fig. $3 A$ ). The forest floor was unburned and covered with living vegetation (fig. $3 B$ ). Lowmoderate-severity sites had some tree mortality (fig. 3C) and incomplete combustion and charring of the organic soils at the forest floor with little or no exposure of mineral soil (fig. $3 D$ ). At high-severity sites, all trees in the area were killed, and downed, woody debris was heavily burned (fig. $3 E$ ). There was complete combustion of organic soil as well as widespread exposure of mineral soil (fig. $3 F$ ).

At each severity sample site, up to three soil samples were collected at all sites. These samples are (1) surficial organic soil (referred to here as the $\mathrm{O}$ horizon), not including living plants, fresh litter, or woody debris; (2) topmost mineral soil, typically intermixed with organic material (A horizon); and (3) the top of the illuviated soil (topmost B horizon). All samples were collected volumetrically. In addition, C-horizon soils were collected from six of the Section 33 wildfire sites.

Table 1. Timetable for collection of various sample media in Voyageurs National Park, Minn.

\begin{tabular}{|c|c|c|c|c|}
\hline $\begin{array}{l}\text { Media sampling } \\
\text { schedule }\end{array}$ & $\begin{array}{c}2003 \\
\text { Planned Ryan Lake } \\
\text { prescribed burn }\end{array}$ & $\begin{array}{c}2004 \\
\text { Section } 33 \\
\text { wildfire }\end{array}$ & $\begin{array}{c}2005 \\
\text { Section } 33 \\
\text { wildfire }\end{array}$ & $\begin{array}{c}2006 \\
\text { Section } 33 \\
\text { wildfire }\end{array}$ \\
\hline Watershed soils & $\begin{array}{l}\text { Ryan Lake } \\
\text { Brown Lake }\end{array}$ & Shoepack Lake & Shoepack Lake & $\begin{array}{l}\text { Shoepack Lake } \\
\text { Ryan Lake } \\
\text { Brown Lake }\end{array}$ \\
\hline Lake waters & $\begin{array}{l}\text { Ryan Lake } \\
\text { Brown Lake } \\
\text { Peary Lake }\end{array}$ & $\begin{array}{l}\text { Ryan Lake } \\
\text { Brown Lake } \\
\text { Peary Lake } \\
\text { Shoepack Lake }\end{array}$ & $\begin{array}{l}\text { Ryan Lake } \\
\text { Brown Lake } \\
\text { Peary Lake } \\
\text { Shoepack Lake }\end{array}$ & $\begin{array}{l}\text { Ryan Lake } \\
\text { Brown Lake } \\
\text { Peary Lake } \\
\text { Shoepack Lake }\end{array}$ \\
\hline Age-1 yellow perch & $\begin{array}{l}\text { Ryan Lake } \\
\text { Brown Lake } \\
\text { Peary Lake }\end{array}$ & $\begin{array}{l}\text { Ryan Lake } \\
\text { Brown Lake } \\
\text { Peary Lake }\end{array}$ & $\begin{array}{l}\text { Ryan Lake } \\
\text { Brown Lake } \\
\text { Peary Lake } \\
\text { Shoepack Lake }\end{array}$ & $\begin{array}{l}\text { Ryan Lake } \\
\text { Brown Lake } \\
\text { Peary Lake } \\
\text { Shoepack Lake }\end{array}$ \\
\hline
\end{tabular}




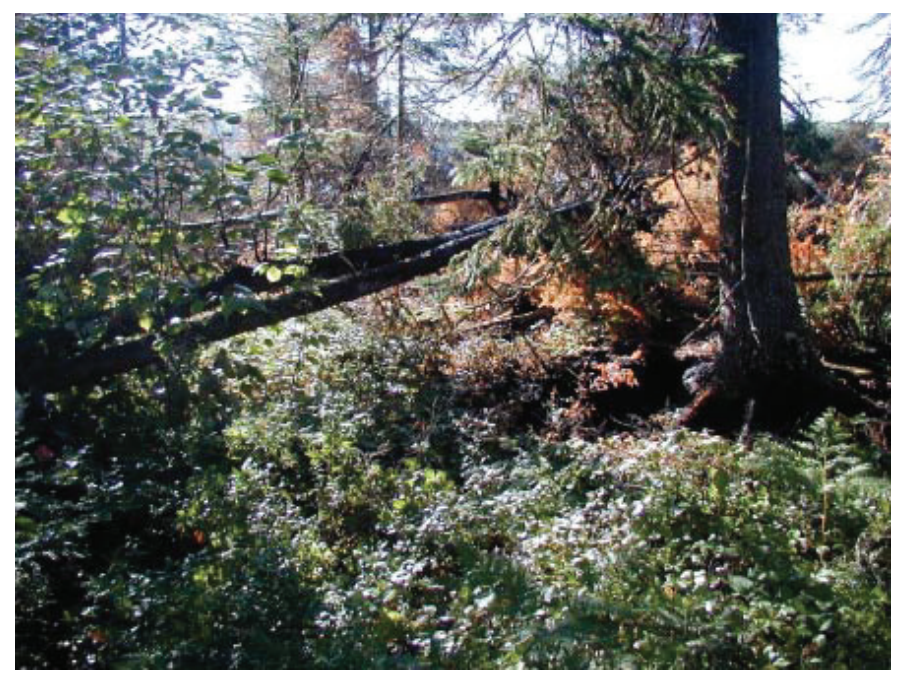

A

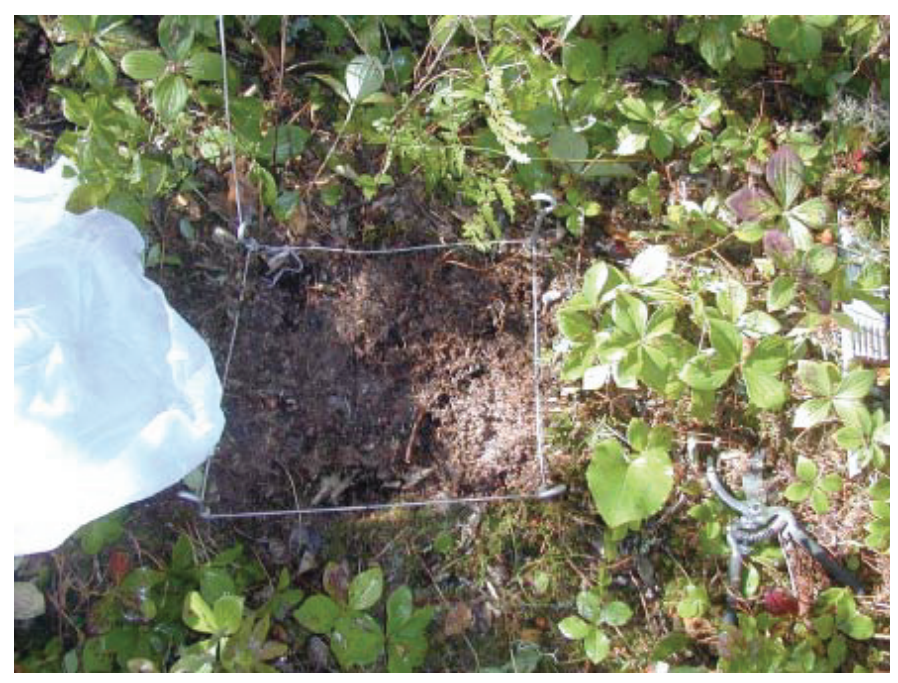

$B$

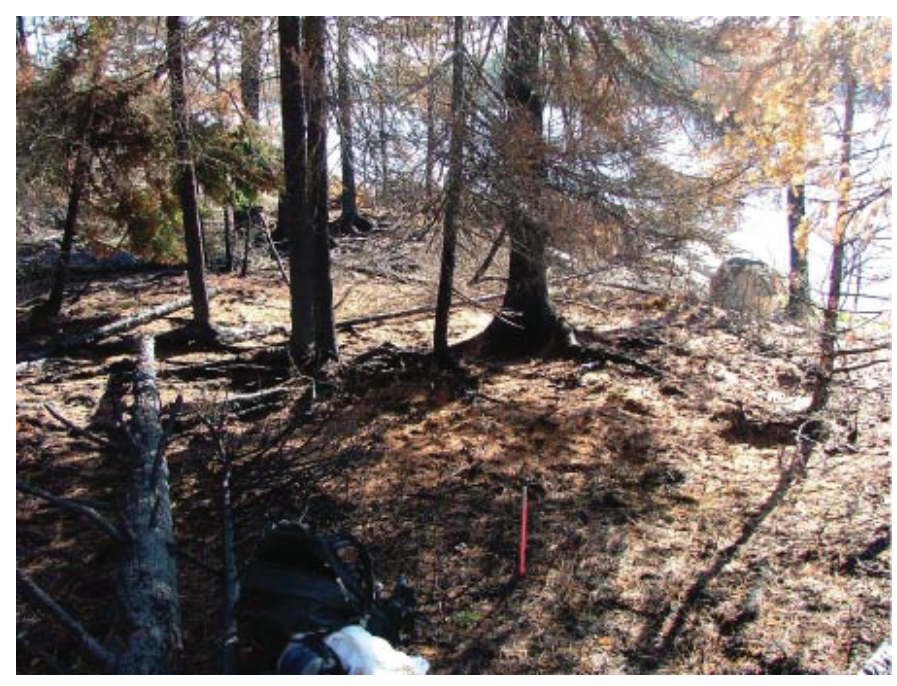

C

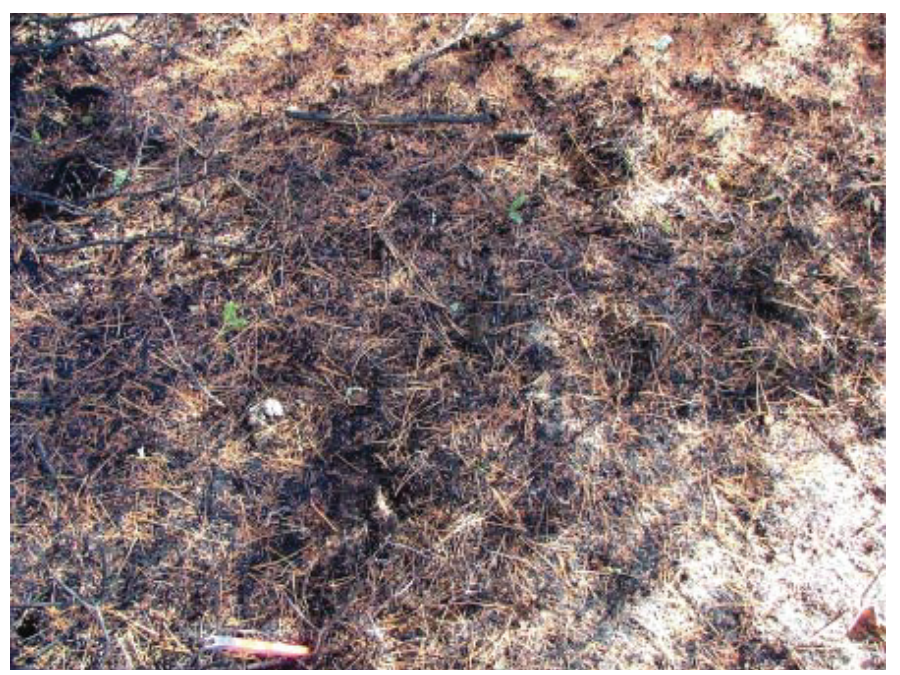

D

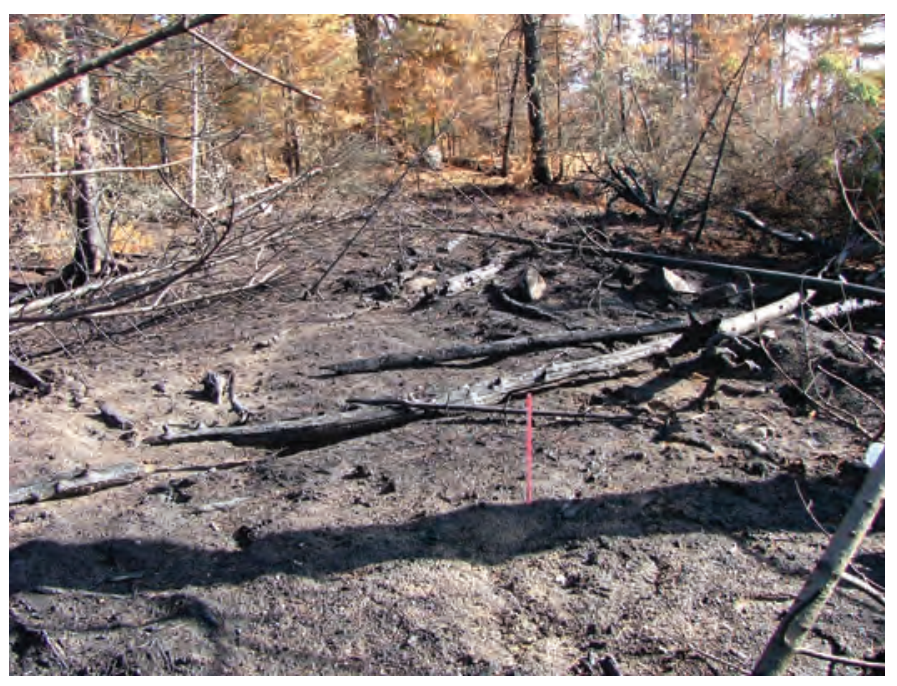

E

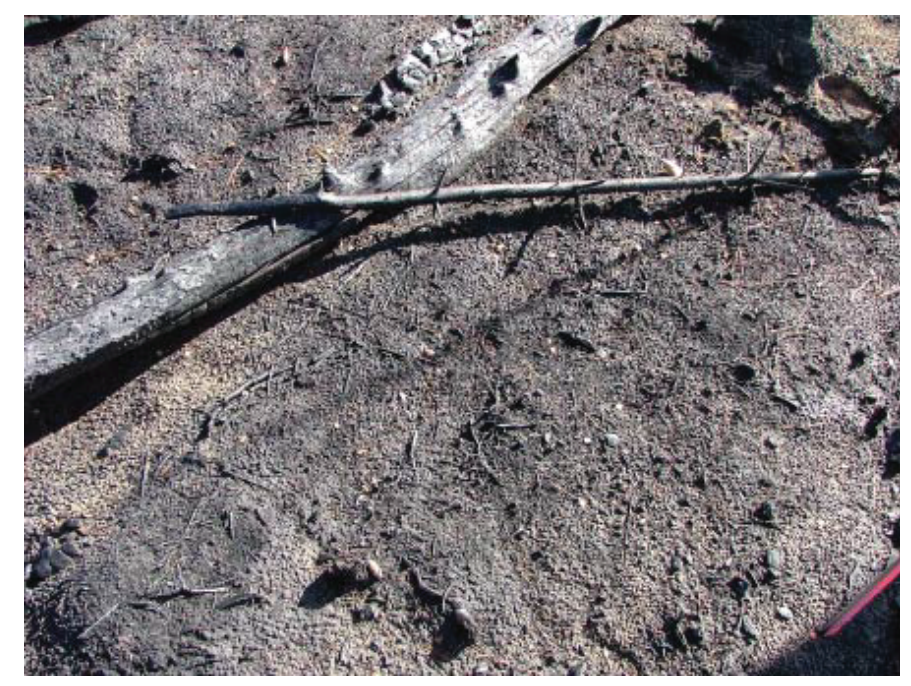

$F$

Figure 3. Photographs of soil sample areas and collection sites characterized by fire severity. $A$ and $B$, site $4-1$, unburned; $C$ and $D$, site 4-2, low-moderate severity with charring of forest floor but no exposure of mineral soil; $E$ and $F$, site 4-3, high severity with complete combustion of 0 horizon and exposure of mineral soil. 
Soil samples were stored in cloth bags (organic soils) or plastic bags (mineral soils) and air dried prior to analysis. Dried soils were sieved to $<2 \mathrm{~mm}$. Soil that passed through the sieve was further ground to $<150 \mu \mathrm{m}$ prior to analyses. O-horizon soils were split, with one portion ashed at $500^{\circ} \mathrm{C}$ for 13 hours. Analytical methods used, including the precision, accuracy, and measurement range for each element in each analytical method, are described in detail in Taggert (2002) and briefly described here. Forty-two major, minor, and trace elements were measured in mineral and ashed soils by a combined method utilizing inductively coupled plasma-atomic emission spectrometry and inductively coupled plasma-mass spectrometry. Selenium was measured on mineral and unashed organic soils using atomic absorption spectrophotometry. Total mercury was determined for mineral and unashed organic soils using a cold vapor technique. Total carbon in mineral soils was determined using an automated carbon analyzer. Percent carbon for the O horizon was calculated as $100-\%$ ash. Samples were submitted to the USGS contract laboratory with a full set of reference standards. Splits of the sieved samples were submitted as blind duplicates at a rate of 1 in 10. A relative percent difference between blind duplicates of less than $25 \%$ was deemed acceptable, as was always the case for mercury and carbon data for this study. Duplicate soil analyses are included in the complete tables in this report's appendixes.

To obtain the mass of the $\mathrm{O}$ horizon, a fixed area was laid out and all decomposed organic-rich material within the area was collected down to the top of the mineral soil. This material was weighed in the field and again after complete air drying to a constant weight. The mass of the $\mathrm{O}$ horizon was calculated as the weight of the dry soil-collected material per unit area. For the A horizon, a fixed area was excavated down to the base of the horizon and all soil was removed. The volume of the excavation was determined by lining the cavity with a plastic bag and filling it with pinto beans to the original ground level. The beans were then weighed, with three replications. The volume of the cavity was later calculated using a formula established in the laboratory that related the weight of the pinto beans to a fixed volume. For the B horizon, 10 short plugs were collected with a small hand metal sampler of known volume. All mineral soil samples were weighed in the field and reweighed following air drying to a constant weight. Mineral soils were hand sieved to $<2 \mathrm{~mm}$ with all soil passing through the sieve reweighed to obtain the total weight of the $<2-\mathrm{mm}$ fraction. The density of the $<2-\mathrm{mm}$ fraction ( $\mathrm{g}$ / $\mathrm{cm}^{3}$ ) was calculated from the weight of the sieved fraction and the calculated volume of the original cavity (A horizon) or metal sampler (B horizon). For A-horizon soils, this density was converted to areal burdens (mass per area; for example, $\mathrm{g} /$ $\mathrm{m}^{2}$ or $\mathrm{mg} / \mathrm{m}^{2}$ ) by dividing by the average depth of the sample cavity.

Soils were categorized by watershed, horizon, fire severity, and year collected. Relationships among geochemical data were analyzed by analysis of variance (ANOVA) methods with Tukey post hoc tests to determine significant varia- tions between means (ANOVA, SYSTAT 11 software, Systat Software, Inc., San Jose, Calif.).

\section{Lake Waters}

Lake-water samples were collected from Shoepack Lake and three control lakes (Ryan, Brown, and Peary), both prefire and post-fire (table 1). Pre-fire water-chemistry data were also available for these and other VNP lakes from an earlier study in the Park (Goldstein and others, 2003). For this study, Ryan, Brown, and Peary Lakes were routinely sampled during 2003,2004 , and 2005. Shoepack Lake was added to the yearly lake-water-sampling schedule in 2004 after the Section 33 fire in the Shoepack Lake catchment and the postponement of prescribed fire in the Ryan catchment. For all lakes, waters typically were sampled in May (a few weeks after ice-out), July, and again in late summer (mid-August to mid-September). In addition, the NPS collected two rounds of samples in 2006 as part of their routine monitoring of VNP lakes. During the NPS sampling, water samples for mercury analysis were collected by protocols used in this study and analyzed by the USGS.

Epilimnetic water from each lake was dip sampled just below the lake surface (approximately $5 \mathrm{~cm}$ ), near the approximate centroid of the lake surface. Sampling was conducted using trace-metal-clean protocols that have been demonstrated to not contaminate samples. Total mercury, methylmercury, elemental mercury, and total organic carbon were analyzed at the USGS Wisconsin Mercury Research Laboratory in Middleton, WI. Total mercury concentrations were determined using USEPA method 1631 (U.S. Environmental Protection Agency, 2002) as modified by Olson and DeWild (1999). Methylmercury concentrations were determined using methods described in DeWild and others (2002). Total mercury values were required to be accurate to within 10 percent of the "true" concentration when concentrations exceeded $0.4 \mathrm{ng} / \mathrm{L}$, 10 times the method detection limit (MDL), and methylmercury values were required to be accurate to within 10 percent of the "true" concentration when concentrations exceeded $0.2 \mathrm{ng} / \mathrm{L}$ (as mercury), approximately 10 times the MDL of $0.025 \mathrm{ng} / \mathrm{L}$. Total organic carbon concentrations were determined using a carbon analyzer using Standard Method 5301D (American Public Health Association and others, 1998). Major ions were analyzed at the USGS National Water Quality Laboratory in Lakewood, CO, using methods of Fishman and Friedman (1989). The laboratory maintains quality objectives through the use of standard reference water samples that constitute a minimum of 10 percent of the analyses and have published acceptance ranges that must be met.

For Shoepack Lake, pre-fire and post-fire conditions were compared using the t-test (PROC TTEST, SAS 9.13 software, SAS Institute, Cary, NC). Time trends for all lakes were tested by regression of log-transformed concentrations vs. time, in years. Because some concentration data were censored (reported as less than the method detection limit), maximum likelihood regression was used (PROC LIFEREG, SAS 9.13) 
to properly account for censoring. The log transformation removed moderate heteroscedasticity in the data.

\section{Age-1 Yellow Perch}

One-year-old yellow perch were sampled shortly after ice-out in May 2003, 2004, 2005, and 2006 from Brown Lake, Peary Lake, and Ryan Lake, and in May 2005 and 2006 from Shoepack Lake (table 1). Fish were collected with seines and a small electroshocker fished in littoral habitat. Each fish was measured [total length (TL) to $1 \mathrm{~mm}$ ], weighed (to $0.01 \mathrm{~g}$ ), and stored at $-30^{\circ} \mathrm{C}$ in a labeled plastic bag until lyophilized at $-85^{\circ} \mathrm{C}$ to a constant dry weight. The age of each fish was determined by examination of three or more scales taken from the area of insertion of the left pectoral fin (DeVries and Frie, 1996). Freeze-dried tissue was homogenized in a stainlesssteel blender. We digested 0.10 -g subsamples with a modification of USEPA method 1631 (U.S. Environmental Protection Agency, 2001). Subsamples were digested for 3 hours at $95^{\circ} \mathrm{C}$ in a solution of sulfuric acid and nitric acid, followed by digestion with bromine monochloride $(\mathrm{BrCl})$ at $40^{\circ} \mathrm{C}$ for 8 hours. Each digestate was analyzed by flow injection cold-vapor atomic fluorescence spectroscopy with a Leeman Labs Hydra AF Gold Plus Mercury Analyzer.

Method precision (relative standard deviation) from triplicate analyses of homogenized fish averaged $6.4 \%$ (table 2). Mean recovery of mercury from fish samples spiked before digestion was $94.5 \%$. Mean measured concentrations of total mercury in three standard reference materials were within or very near the certified ranges, which ranged from 57.4-64.6 to $724-872 \mathrm{ng} / \mathrm{g}$ dry weight (table 3 ). The accuracy of mercury determinations for each batch of fish samples was verified by the concomitant analyses of (1) certified reference materials from the U.S. National Institute of Standards and Technology (NIST) and the National Research Council of Canada (NRCC), (2) triplicate subsamples of homogenized fish, (3) spiked (before digestion) subsamples of homogenized fish, and (4) blanks and standards taken through the digestion procedures. Concentrations of total mercury in all fish samples exceeded our estimated method detection limit of $1.70 \mathrm{ng} / \mathrm{g}$ dry weight.

In order to determine if mercury concentrations and growth of yellow perch from Shoepack Lake had changed as results of the fire in 2004, we used analysis of variance with a "beyond Before-After-Control-Impact (BACI)" design (Underwood, 1992; 1994). This design required data on yellow perch from Shoepack Lake and control lakes (Brown, Peary, and Ryan) prior to 2005. Data on TL and mercury concentrations in age-1 yellow perch were obtained from all four lakes in 2000 and 2001 in conjunction with another project within VNP (Wiener and others, 2006). Therefore, we used data on yellow perch collected in 2000 and 2001 (before the fire) and in 2005 and 2006 (after the fire) for the analysis of variance.

Table 2. Summary results of quality-assurance analyses during determination of total mercury in samples of yellow perch sampled in 2003-2006 from Brown, Peary, Ryan, and Shoepack Lakes in Voyageurs National Park, Minn.

\begin{tabular}{|c|c|c|}
\hline Material analyzed & Performance measure & Our results \\
\hline Standard reference materials & Measured concentrations within the certified range & 51 of 66 \\
\hline Fish tissue spiked before digestion & $\begin{array}{l}\text { Percent recovery: } \\
\text { mean } \\
\text { range }\end{array}$ & $\begin{array}{l}94.5 \% \\
74.6 \%-117 \%\end{array}$ \\
\hline Triplicate subsamples of fish & $\begin{array}{l}\text { Method precision (coefficient of variation): } \\
\text { mean } \\
\text { range }\end{array}$ & $\begin{array}{l}6.1 \% \\
0.1 \%-17.7 \%\end{array}$ \\
\hline
\end{tabular}

Table 3. Analytical results for total mercury in reference materials from the U.S. National Institute of Standards and Technology and the National Research Council of Canada.

[Reference materials were analyzed with samples of yellow perch from 2003 through 2006 in Brown, Peary, Ryan, and Shoepack Lakes, Voyageurs National Park, Minn. Ng/g, nanograms per gram]

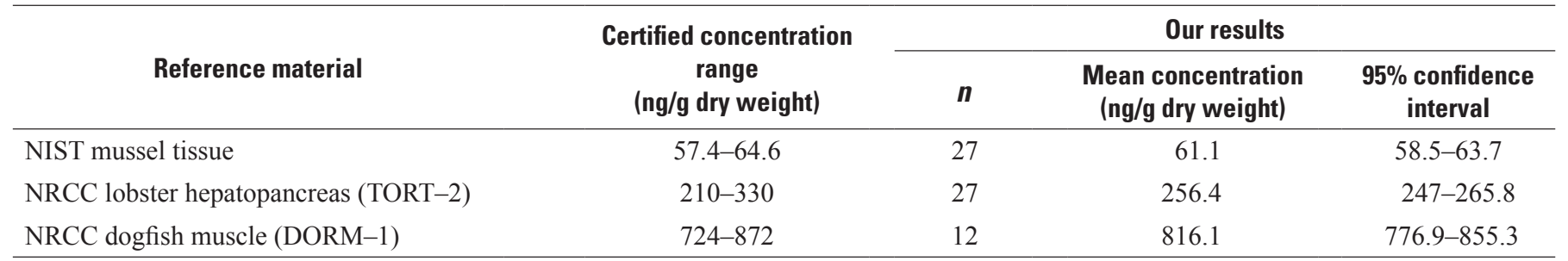




\section{Results and Discussion}

\section{Soils}

Complete datasets for all soils collected from the Shoepack Lake watershed within the Section 33 wildfire footprint and from the Ryan Lake and Brown Lake watersheds are reported in appendixes 1 through 7. Data are given in weight percent (wt. \%) or parts per million (ppm). All three study lakes are underlain by Precambrian bedrock consisting of fineto medium-grained biotite schist, often cut by pegmatite dikes (Day and others, 1990). Soils in the study areas are typically sandy loams developed on thin glacial deposits largely derived from material similar in composition to underlying metamorphic rocks (Erickson, 1981). A soil pit from the Ryan Lake watershed in VNP is shown in figure 4. A typical soil profile in VNP has an O horizon of decomposing organic material overlying an A horizon, a dark brown to black mineral soil with some organic matter accumulation. Some soils in VNP have very thin or no A horizon and an O horizon may sit directly on an E horizon, a pale gray mineral soil leached of many mineral and organic constituents. The B-horizon subsoil often is reddish because of subsurface accumulation of clays or iron, while the $\mathrm{C}$ horizon is little-weathered soil parent material.

The chemistry of B- and C-horizon soils collected from the three study watersheds plot along consistent trends expected for soils derived from similar parent mineralogy, principally plagioclase, quartz, and micas. There were no significant differences in soil parent chemistry noted among the three watersheds. C-horizon soils from the Shoepack Lake watershed have mercury concentrations that range from $<0.02$ ppm $\mathrm{Hg}$ (the lower method detection limit) to $0.03 \mathrm{ppm} \mathrm{Hg}$, notably lower than the average concentrations of mercury in unburned O-horizon (0.14 ppm $\mathrm{Hg}$ ) and A-horizon soils (0.15 ppm Hg). This supports the conclusion of Wiener and others (2006) that geologic sources of mercury in VNP soils are apparently insignificant.

\section{Preburn Soil Chemistry}

Mercury in soils is typically bound to organic carbon. Because soils from the Ryan Lake and Brown Lake watersheds had no statistically significant differences in soil

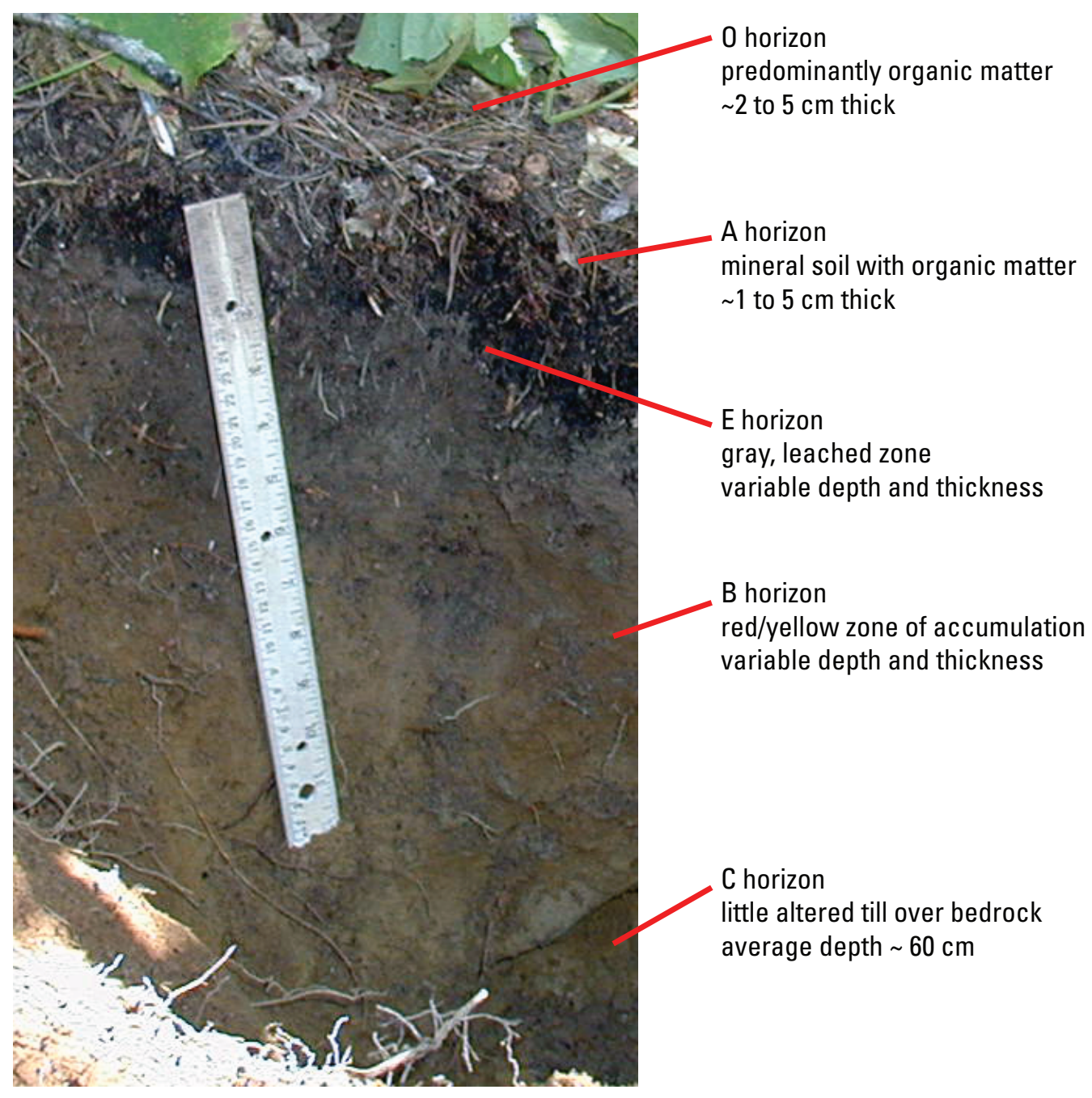

Figure 4. Soil pit from the Ryan Lake watershed in Voyageurs National Park showing different soil horizons. 
chemistry between samples collected in 2003 and samples collected in 2006, data from both years were combined for comparisons. The distributions of mercury and carbon in A and B horizons for soils from the two control watersheds and for unburned sites in the Section 33 fire area are shown in figures $5 A$ and $5 B$. A-horizon soils typically have much higher mercury concentrations than B-horizon soils. A- and B-horizon soils also have the good correlation of mercury with carbon observed for many forest soils (Grigal, 2002) (fig. 5C).

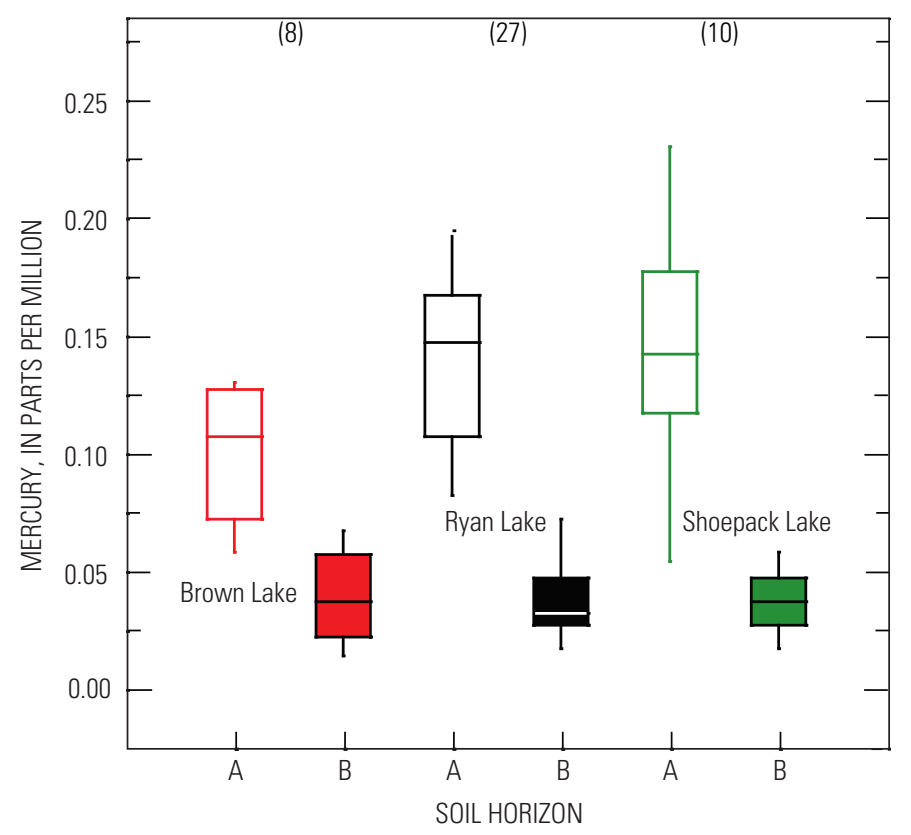

$A$

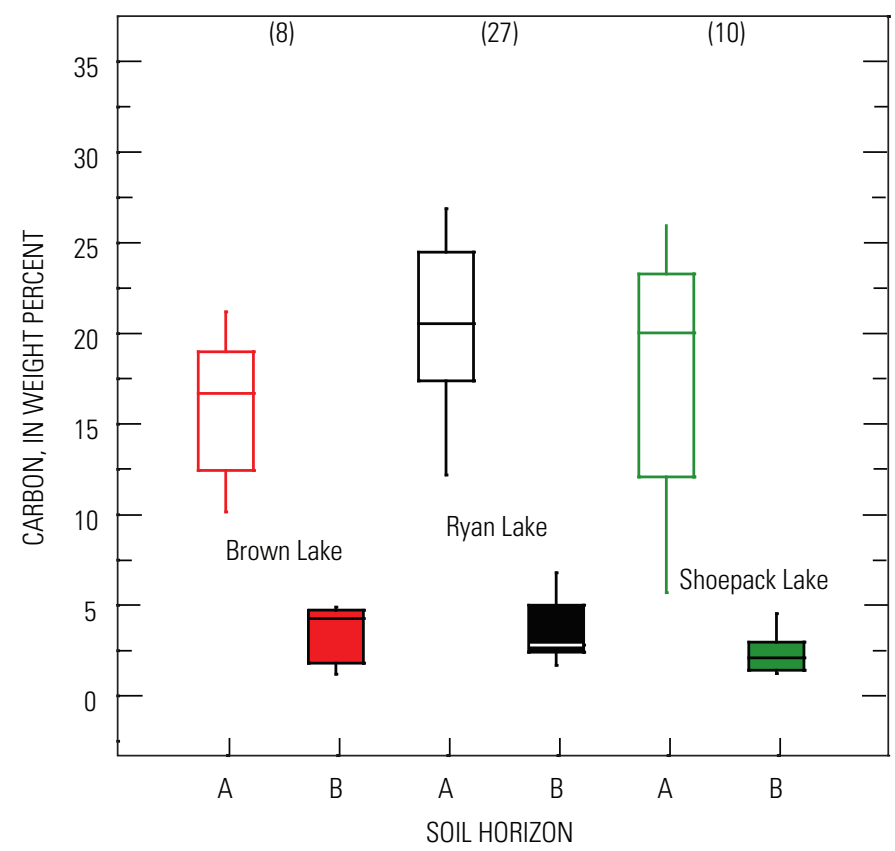

$B$
ANOVA tests showed no significant differences for concentrations of mercury, or any other elements, between soils from equivalent horizons in unburned Shoepack Lake watershed sites and control sites from the Ryan Lake and Brown Lake watersheds, suggesting that the unburned sites at Shoepack Lake can be used as "pre-fire" control sites for the fire-severity comparisons that follow.

Concentrations and the areal burdens of mercury in the three soil horizons collected from 10 unburned sites from

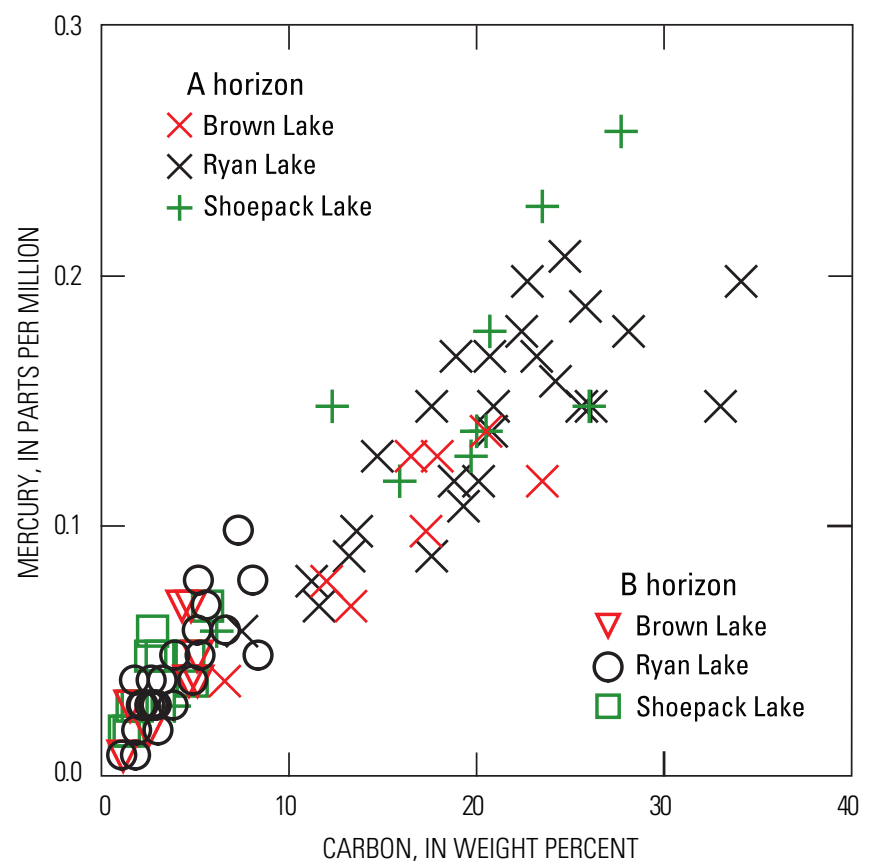

C

EXPLANATION

(n) Sample

population size

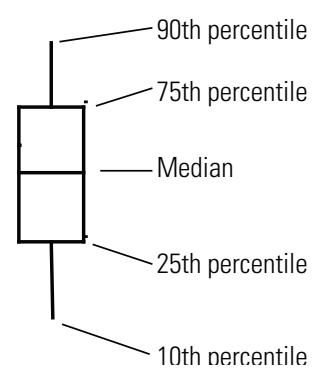

Figure 5. Box plots of $A$, mercury, and $B$, carbon concentrations in $A$ - and B-horizon soils from Brown Lake and Ryan Lake watersheds collected in 2003 and 2006 and from the unburned sites from the Shoepack Lake watershed collected in 2004. C, The relationship between mercury and carbon in both horizons. Number in parentheses is number of samples used for each plot. 
Shoepack Lake are shown as box plots in figure 6. Although organic O-horizon soils typically have high concentrations of mercury, predominantly mineral soils will contain the larger masses of mercury because of their higher densities. Therefore, while an $\mathrm{O}$ horizon is more combustible than underlying mineral soils and so represents a major source of mercury potentially mobilized by fire, A-horizon soils, if sufficiently heated, could be a more significant mercury source.

\section{Soil Chemistry along Gradients of Fire Severity in 2004}

Concentrations and calculated areal burdens for carbon and mercury in both O- and A-horizon soils collected from similar fire-severity gradients in 2004 from the Section 33 fire footprint are given in figure 7. By our severity classification, all O-horizon soils were totally combusted at high-severity sites and all contained mercury was released. The fire's impact on mercury contained in A-horizon soils is variable among the sampled areas (table 4). Two sites (sites 1 and 5) show little effect of fire on mercury in minerals soils, but data from the other eight sites indicate significant mercury mobilization from high-severity sites and possibly slight losses from low-moderate-severity sites. A plot of mercury vs. carbon in A-horizon soils shows that both elements were mobilized at high-severity sites (fig. 8). ANOVA testing of A-horizon soils across the fire-severity gradient indicates that concentrations and areal burdens of mercury and carbon are statistically significantly lower in high-severity sites compared to both lowmoderate-severity and unburned sites. However, the differences in mercury and carbon between low-moderate-severity sites and unburned sites are not statistically significant.

Table 4. Mercury concentrations in A-horizon soils at 10 sites sampled in 2004 within Section 33 wildfire areas. Soils were collected across gradients of fire severity.

[Descriptions of fire severity criteria are given in the text. ppm $\mathrm{Hg}$, parts per million mercury]

\begin{tabular}{lccc}
\hline \multirow{2}{*}{$\begin{array}{c}\text { Section 33 } \\
\text { sample area }\end{array}$} & $\begin{array}{c}\text { Fnburned } \\
\text { (ppm Hg) }\end{array}$ & $\begin{array}{c}\text { Low-moderate } \\
\text { (ppm Hg) }\end{array}$ & $\begin{array}{c}\text { High } \\
\text { (ppm Hg) }\end{array}$ \\
\cline { 2 - 4 } Site 1 & 0.03 & 0.05 & 0.03 \\
Site 2 & 0.03 & 0.16 & 0.23 \\
Site 3 & 0.04 & 0.01 & 0.15 \\
Site 4 & 0.03 & 0.19 & 0.26 \\
Site 5 & 0.04 & 0.04 & 0.06 \\
Site 6 & 0.01 & 0.09 & 0.18 \\
Site 7 & 0.01 & 0.19 & 0.14 \\
Site 8 & 0.08 & 0.04 & 0.12 \\
Site 9 & 0.02 & 0.11 & 0.14 \\
Site 10 & 0.01 & 0.11 & 0.15 \\
\hline
\end{tabular}

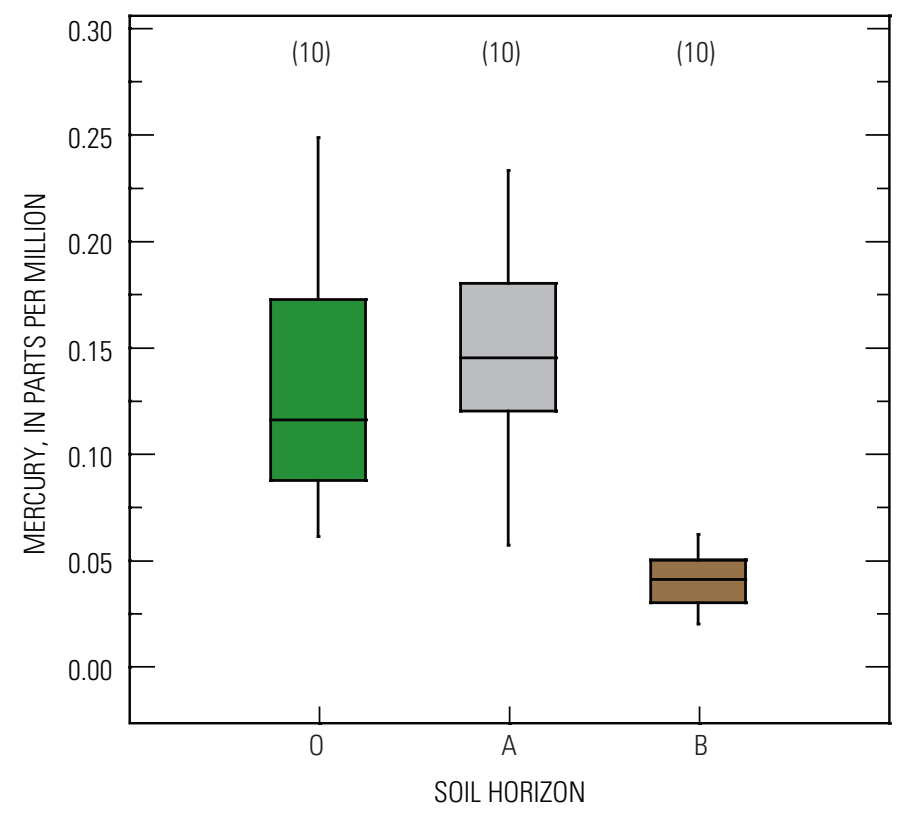

$A$

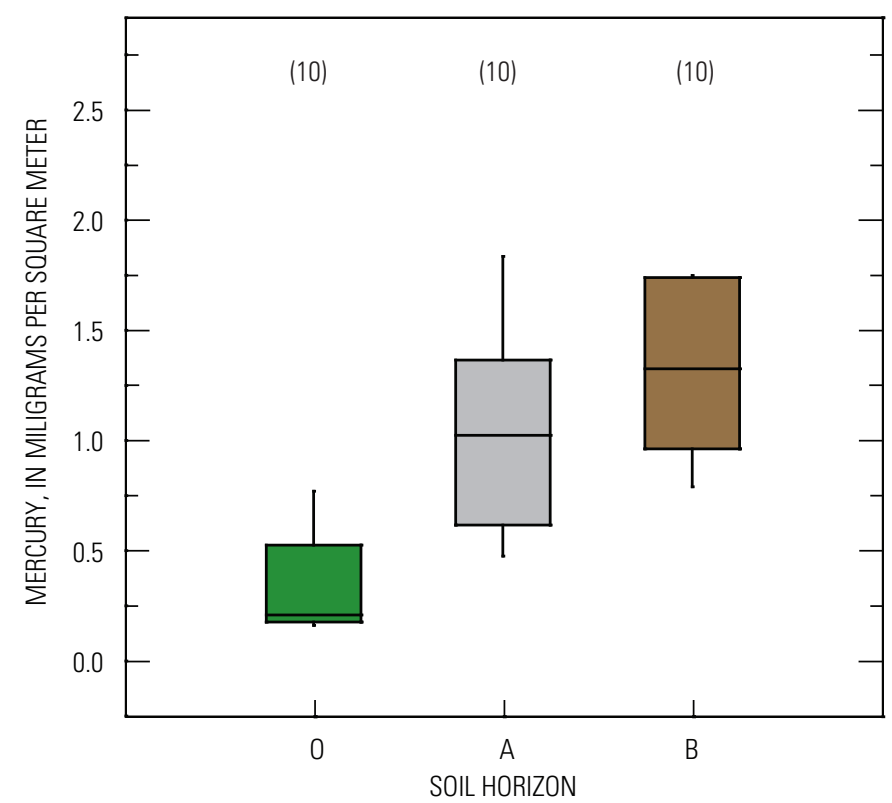

$B$

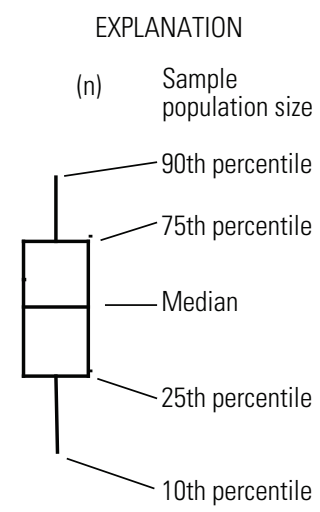

Figure 6. Box plots of $A$, concentrations, and $B$, calculated areal burden (mass per unit area) for mercury in $0-$, $\mathrm{A}-$, and $\mathrm{B}$-horizon soils from unburned sites within the Section 33 wildfire footprint in the Shoepack Lake watershed. Number in parentheses is number of samples used for each plot. 


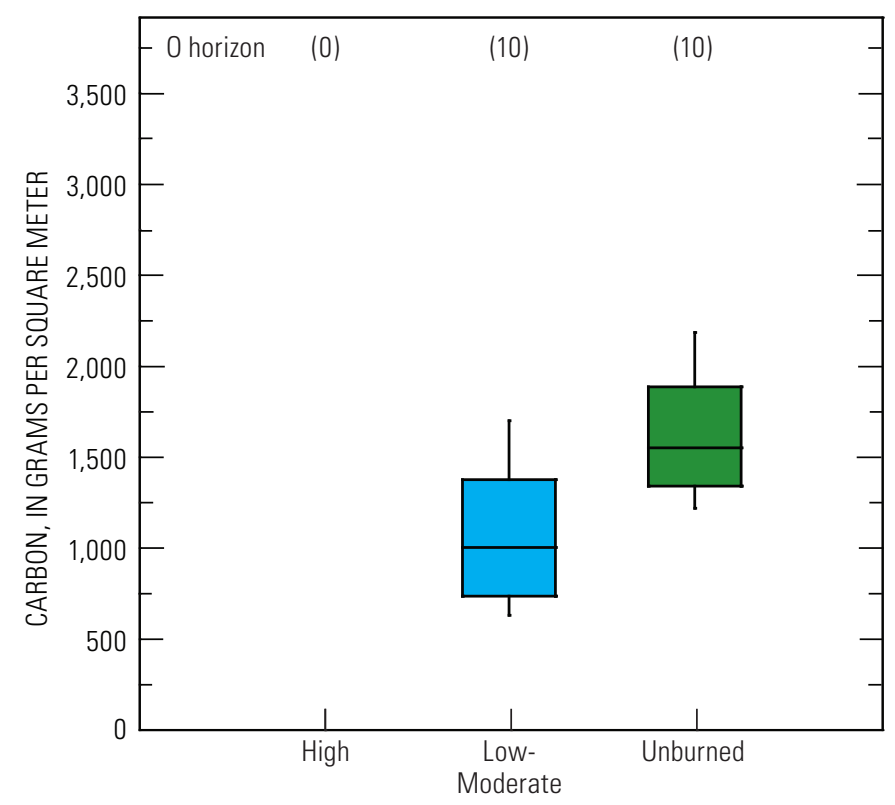

FIRE-SEVERITY CLASSIFICATION

$A$

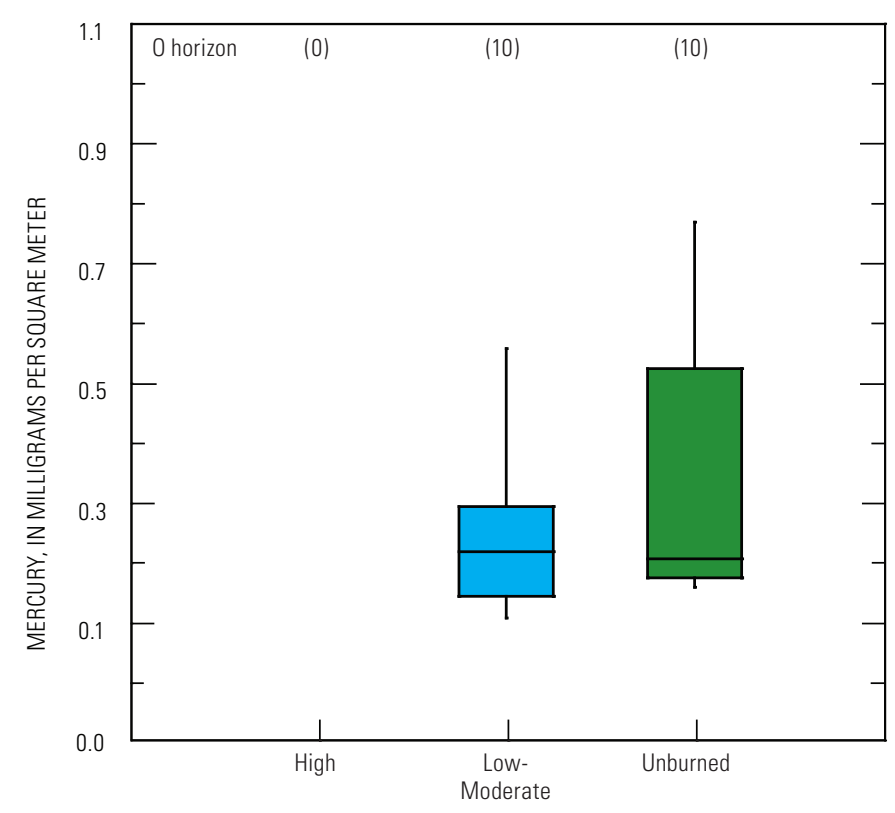

FIRE-SEVERITY CLASSIFICATION

$B$ EXPLANATION

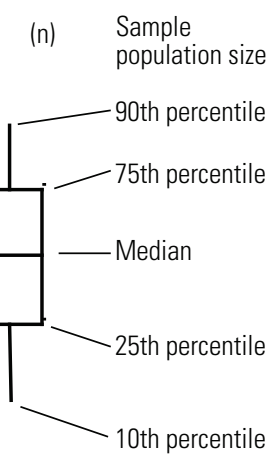

Figure 7. Box plots of calculated areal burdens for carbon and mercury in 0- and A-horizon soils from sample sites within the Section 33 wildfire footprint in the Shoepack Lake watershed collected across gradients of fire severity. Number in parentheses is number of samples used for each plot.

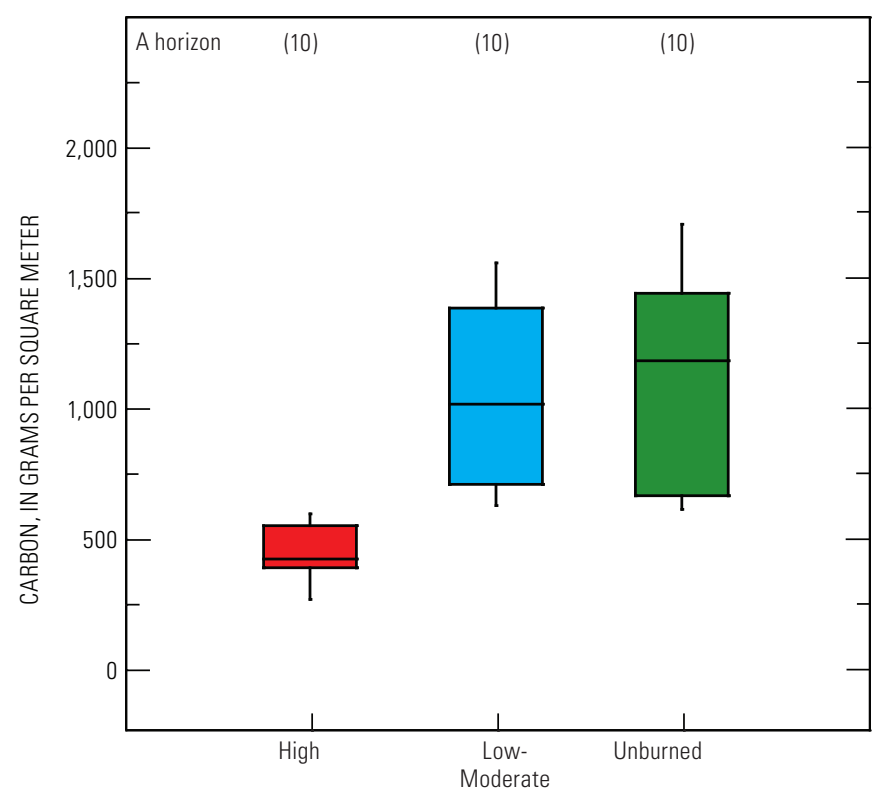

FIRE-SEVERITY CLASSIFICATION

C

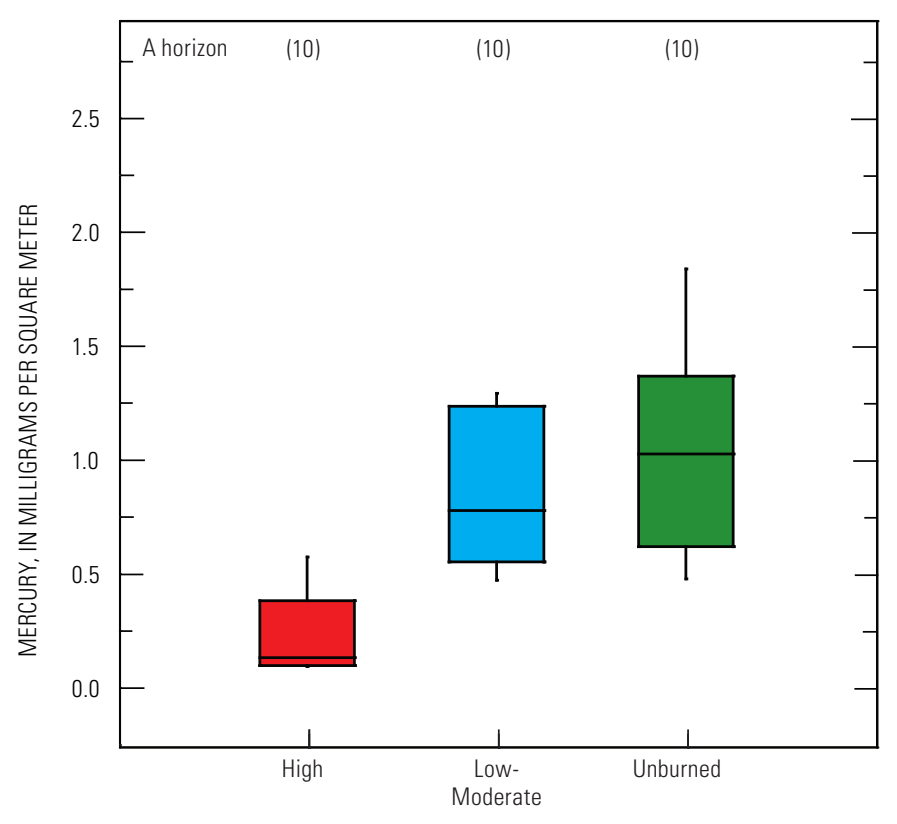

FIRE-SEVERITY CLASSIFICATION

$D$

The mobilization of mercury from mineral soils observed for the Section 33 wildfire high-severity sites is an important observation on the fate of mercury during wildfire. While mineral soils are the largest pool of mercury sequestered in soils (Grigal, 2002), few studies have quantified mercury emissions from mineral soils following fire (Engle and others, 2005; Biswas and others, 2007). Results from this study show that the Section 33 wildfire created conditions of sufficient intensity and duration to release some fraction of sequestered mercury and carbon in uppermost mineral soils. 


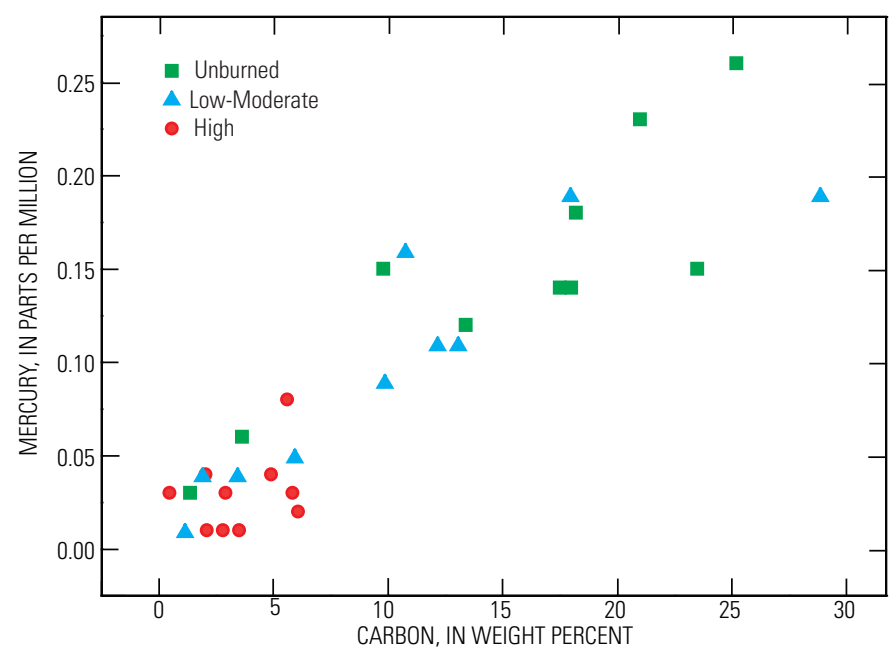

Figure 8. Plot of mercury versus carbon for A-horizon soils collected in 2004 across fire-severity gradients in Section 33 wildfire footprint in Shoepack Lake watershed.

Median values for areal burdens of mercury and carbon in O-horizon and A-horizon soils collected in 2004 are given in table 5. If the unburned sites from the Shoepack Lake watershed are representative of average mercury concentrations of the forest soils prior to the ignition of the Section 33 wildfire, these data suggest that at sites where all the organic forest floor material was combusted, exposing mineral soils (equivalent to high-severity sites), approximately $0.21 \mathrm{mg} \mathrm{Hg} / \mathrm{m}^{2}$ was potentially liberated from combusted organic material during the fire. For A-horizon soils, the difference between unburned and high-severity sites suggests potential mobilization of about $0.89 \mathrm{mg} \mathrm{Hg} / \mathrm{m}^{2}$. Based on these values, total mercury mobilization from soils within areas of high-severity fire in the Section 33 wildfire was about $1.1 \mathrm{mg} \mathrm{Hg} / \mathrm{m}^{2}$. This is similar to mercury emissions of 0.26 to $0.86 \mathrm{mg} \mathrm{Hg} / \mathrm{m}^{2}$ estimated by Turetsky and others (2006) for fire events in upland forests, but less than their estimates of 2 to $7 \mathrm{mg} \mathrm{Hg} / \mathrm{m}^{2}$ for fire events in boreal forest regions.

Fire severity was the primary control on mercury mobilization from the Section 33 wildfire, similar to findings from other studies (for example, Harden and others, 2004; Amirbahman and others, 2004; Kelly and others, 2006). Fire severity maps based on satellite imagery were constructed for the Section 33 fire by the USGS and the NPS. These maps show that approximately $4.047 \mathrm{~km}^{2}$ were burned in the fire; vegetation mapping suggests that the landscapes within the burn perimeter could be characterized as about half upland and half wetland areas. On the basis of satellite imagery, it is estimated that about $75 \%$ of the upland areas and none of the wetland areas could be classified as high severity, giving a total of about $1.52 \mathrm{~km}^{2}$ burned in high-severity settings (S. Weyenbery, NPS, personal communication, 2007). Using our calculation of mercury losses from high-severity burned soils of about $1.1 \mathrm{mg} \mathrm{Hg} / \mathrm{m}^{2}$, we estimate that a total of $\sim 1.6$ $\mathrm{km}$ of mercury were potentially mobilized from soils within the Section 33 wildfire footprint.
Table 5. Median areal burdens for mercury and carbon in 0 -horizon and A-horizon soils collected in 2004 within Section 33 wildfire areas.

[Soils were collected across gradients of fire severity. Descriptions of fireseverity criteria are given in text. $\mathrm{g} / \mathrm{m}^{2}$, grams per square meter; $\mathrm{mg} / \mathrm{m}^{2}$, micrograms per square meter]

\begin{tabular}{lcr}
\hline \multirow{2}{*}{$\begin{array}{c}\text { Areal burdens and fire } \\
\text { severity }\end{array}$} & \multicolumn{2}{c}{ Soil horizon } \\
\cline { 2 - 3 } & O horizon & A horizon \\
\hline Mercury $\left(\mathrm{mg} / \mathrm{m}^{2}\right):$ & 0.00 & 0.13 \\
High & 0.22 & 0.77 \\
Low-moderate & 0.21 & 1.02 \\
Unburned & & \\
Carbon $\left(\mathrm{g} / \mathrm{m}^{2}\right):$ & 0 & 420 \\
High & 1,131 & 1,013 \\
Low-moderate & 1,374 & 1,180 \\
Unburned & & \\
\hline
\end{tabular}

\section{Soil Chemistry from 2004 to 2006}

Soils were collected in successive years from areas as close as possible to the original sample sites, always within 1 to $2 \mathrm{~m}$ of the stake marking the 2004 site. Typical site regeneration at high-severity sites over the 2-year timeframe of the study is shown in figure 9. In May 2005, 9 months following the fire, severe sites often had a mat of liverwort growing on exposed mineral soil. By May 2006, liverwort had often disappeared, replaced by mosses and grasses. Variations with time for all three severity classifications for mercury and carbon are given in figure 10. ANOVA testing found no significant trends for mercury or carbon for soils from the same horizon collected in close proximity to one another with the same fire severity classification over the different years. It was noted in the field that there was a decrease in the quantity of O-horizon soils collected within a fixed area for sites classified as lowmoderate severity. In 2004, O-horizon samples were collected from all 10 sites. In 2005, O-horizon samples were collected from eight sites, but by 2006 only two of the sites still had sufficient quantities of organic material to collect (fig. 10). The cause of this depletion in $\mathrm{O}$ horizon is not known.

\section{Lake Waters}

This section reports on methylmercury in unfiltered water and related concentrations of total mercury in unfiltered water, and total organic carbon. Complete water-quality data are reported in appendix 8. Concentrations of mercury and methylmercury are given in units of nanograms per liter (ng/L), equivalent to parts per trillion.

Aqueous concentrations of methylmercury are a key indicator of exposure of aquatic food webs to methylmercury, and thus aqueous methylmercury is a key species of interest in studies of mercury in natural waters. Because water-column data were compiled from several sampling efforts extending 


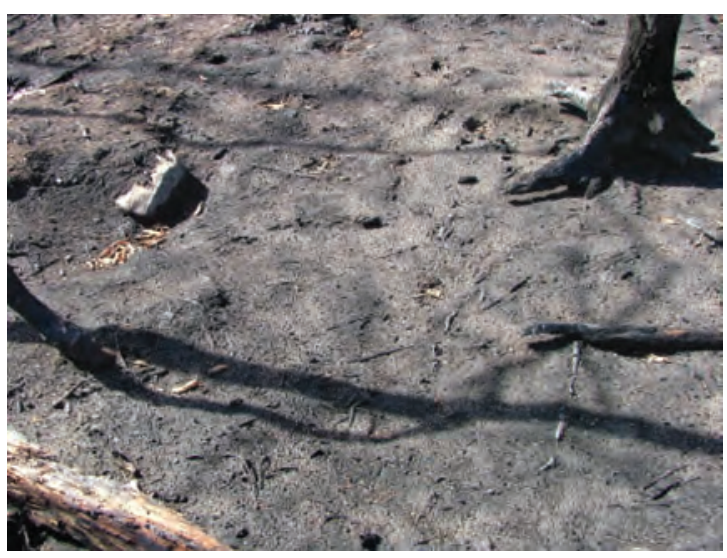

\section{Shoepack Lake Waters-Comparison of Pre-Fire and Post-Fire Conditions}

The distribution of methylmercury concentrations was somewhat lower in Shoepack Lake in the post-fire period (2004-2006) than in the pre-fire period (2000-2003). This difference was significant (two-tailed t-test; $p=0.03$ ). The mean difference was $0.0816 \mathrm{ng} / \mathrm{L}$, which is 28 percent of the mean pre-fire methylmercury concentration of $0.283 \mathrm{ng} / \mathrm{L}$. For reasons discussed earlier, readers are cautioned to not attach too much weight to statistical summaries of this small dataset. However, methylmercury appears to have significantly decreased in Shoepack lake waters rather than increased, relative to pre-fire conditions, and the lack of a sharp increase in aqueous methylmercury shortly following a significant

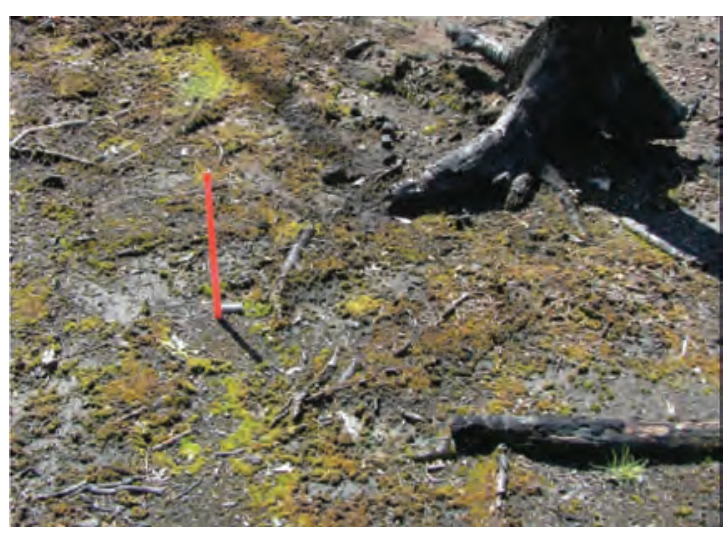
wildfire is an important finding for natural resource managers. Aqueous total mercury and total organic carbon concentrations in Shoepack Lake were not significantly different post-fire compared to pre-fire.

\section{Shoepack Lake Waters Compared to Control Lakes}

The suggestion of a step decrease in aqueous methylmercury concentrations in Shoepack Lake should be viewed in the context of the three control lakes-Ryan, Brown, and Peary Lakes. Three of the four sampled lakes, including Shoepack Lake, had downward trends in aqueous methylmercury concentrations over the period from 2000-2006 (fig. 11). When data for all four lakes were combined, the trend in aqueous methylmercury is still significant $(p=0.008)$. Given that two of the three control lakes (Ryan and Peary) had downward trends, similar to Shoepack Lake, the downward trend in Shoepack Lake cannot be attributed to the fire in that lake's catchment. It is more likely that lakes in the Park were responding to another factor or set of factors, such as decreased atmospheric mercury inputs or changes in regional precipitation patterns, which could affect both mercury methylation rates and inputs from connected wetlands to lakes.

\section{Age-1 Yellow Perch}

Figure 9. Photographs showing vegetation regeneration from high-severity soil site 6-3 following the Section 33 wildfire. $A$, August 2004, 2 weeks following the fire; $B$, May 2005, 9 months after the fire; $C$, May 2006, 17 months after the fire.

both before (2000-2002) and after (2006) the planned study, and also because of the unplanned Section 33 wildfire replacing the originally planned Ryan Lake prescribed fire, there are some year-to-year variations in sampling regime (table 3). Because of these variations and the relatively short duration of data collection, statistical analyses should be interpreted with due caution.

A total of 384 yellow perch from the four study lakes collected in 2003, 2004, 2005, and 2006 were analyzed (table 3). The sample size for individual lakes ranged from 11 to 47 fish (median, 30). Data are reported in appendix 9 as whole-body concentrations (wet weight) of total mercury in nanograms/gram (ng/g), or parts per billion. The annual mean $\mathrm{TL}$ and mass of age-1 yellow perch were generally consistent within each lake (table 6). Annually, the mean TL and mass of fish were generally least from Brown Lake (50-55 mm TL, $1.08-1.47 \mathrm{~g})$ and greatest from Shoepack Lake (60-67 mm TL, $1.68-2.40 \mathrm{~g})$.

Total mercury concentrations in the yellow perch varied markedly within and among lakes during the 4 years of the 

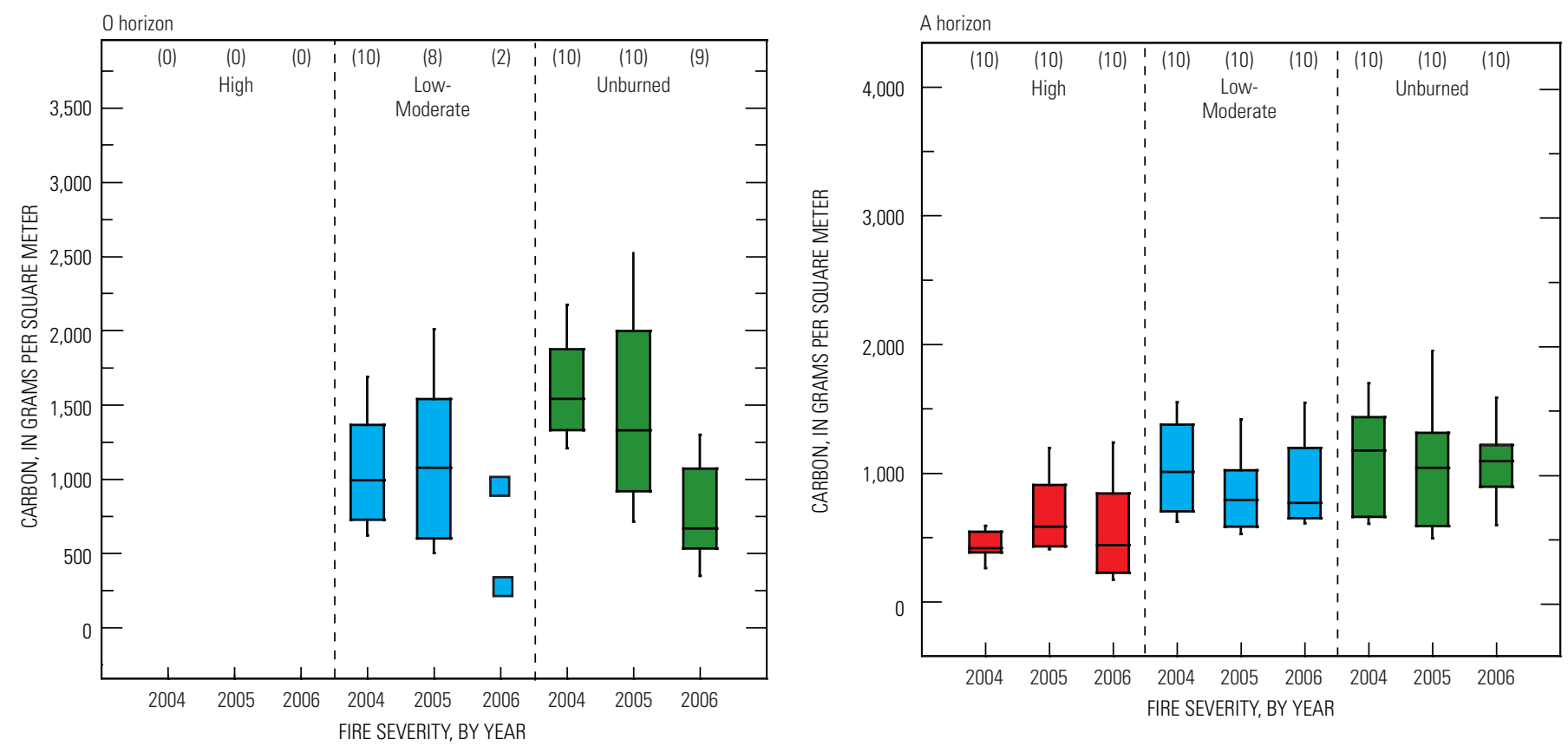

$A$

C
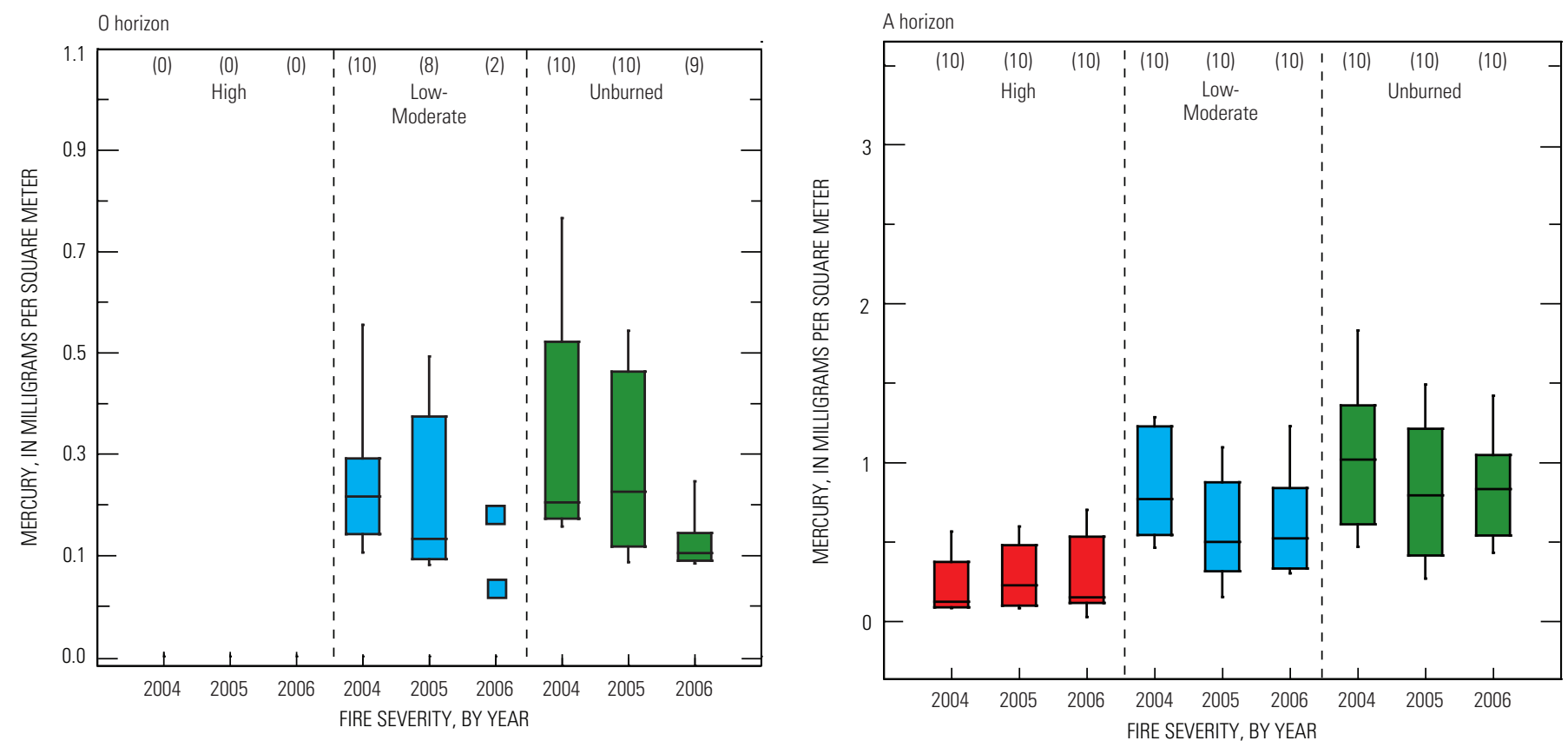

$B$

$D$

EXPLANATION

(n) Sample

population size

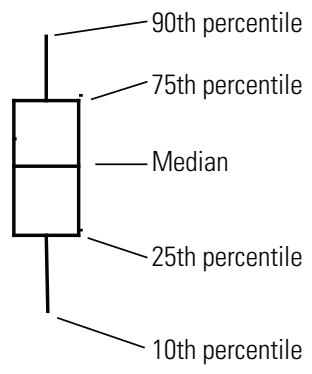

Figure 10. Box plots of calculated loads for carbon and mercury in $A$ and $B, 0$-horizon, and $C$ and $D$, A-horizon soils from sample sites within the Section 33 wildfire footprint in the Shoepack Lake watershed collected over the length of this study. The individual data points for 0-horizon low-moderate sites in 2006 are plotted as filled blue squares. Number in parentheses is number of samples used for each plot. 


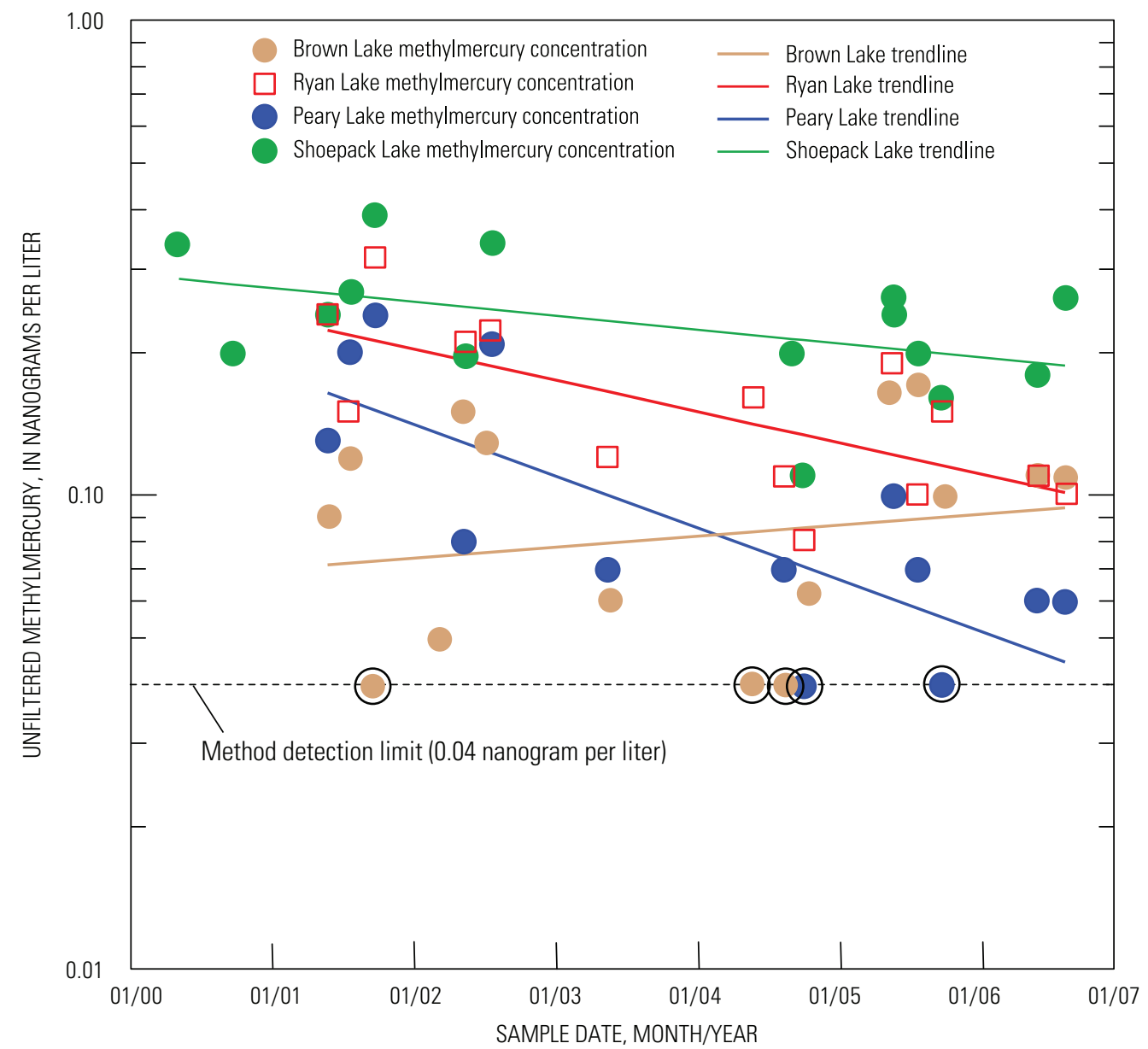

Figure 11. Plot illustrating data and least square regession trendlines in methylmercury concentrations in unfiltered lake waters from the four study lakes. Data points circled have values lower than the method detection limit of 0.04 nanograms per liter. study (table 6 and fig. 12). For example, mean mercury concentrations in yellow perch from Peary Lake varied more than fourfold from $2004(31 \mathrm{ng} / \mathrm{g})$ to $2006(137 \mathrm{ng} / \mathrm{g})$. Mean mercury concentrations were least in yellow perch collected from Peary Lake in $2004(31 \mathrm{ng} / \mathrm{g})$ and greatest in yellow perch collected from Ryan Lake in 2003 and 2004 (180 ng/g).

While there was a significant variation in mercury concentrations for age- 1 yellow perch among the control lakes after the fire (table $7, p<0.0001$ ), there was not a significant difference in concentrations of total mercury in yellow perch from Shoepack Lake in 2005 and 2006 (after fire) relative to that measured during 2000 and 2001 (before fire; table 7, $p>0.57$ ). The effect of fire on concentrations of mercury in aquatic biota is primarily dependent upon the characteristics of the watershed (Allen and others, 2005) and trophic structure of the aquatic ecosystem (Kelly and others, 2006). For example, Garcia and Carignan (2005) examined the relation between mercury concentrations in fish from 38 drainage lakes with clear-cut, burnt, or undisturbed watersheds. Highest mercury concentrations were found in fish from lakes in partially burnt watersheds. However, relative to control lakes in undisturbed catchments, there was no significant enhancement of mercury in fish from lakes within completely burnt watersheds. Similarly, no effects of forest fire on methylmercury concentrations in zooplankton and fish were observed in boreal lakes (Garcia and Carignan, 1999, 2000). Conversely, Kelly and others (2006) found that mercury bioaccumulation in fish increased after fire as a result of an increase in nutrient concentrations that increased productivity and restructured the food web.

Although methylmercury bioavailability may increase after fire, a concomitant increase in nutrient flux to a lake may result in an increased productivity that results in a reduction in methylmercury in fish via biodilution or growth dilution (Allen and others, 2005). There was not a detectable difference in the growth of age-1 yellow perch in Shoepack Lake between years (table 7, $p>0.31$ ); hence, the data suggest that the lack of change in methylmercury concentrations in yellow perch from Shoepack Lake was the result of a lack of effect of fire on the bioavailability of methylmercury in the lake rather than growth dilution.

\section{Summary and Conclusions}

Analyses of soils for this study confirm that in VNP, mercury is deposited across the landscape by atmospheric deposition. Thus, variations in the distribution of mercury in soils across the Park are the result of differences in mercury delivery to the forest floor and processes that can affect mercury cycling in soils and not of differences in soil parent 
Table 6. Characteristics of age-1 yellow perch sampled from Brown, Peary, Ryan, and Shoepack Lakes in Voyageurs National Park in May 2003-2006.

[Perch sampled in May for each of the years from 2000 through 2006; Shoepack Lake was not sampled in 2003 and 2004. g, grams; Hg, mercury; Max, maximum value; Min, minimum value; mm, millimeters; NA, not available; $n$, sample size (the number of fish); ng/g, nanograms per gram; SE, standard error of the mean]

\begin{tabular}{|c|c|c|c|c|c|c|c|c|c|c|c|c|c|}
\hline \multirow[b]{2}{*}{ Year } & \multirow[b]{2}{*}{ Statistic } & \multicolumn{3}{|c|}{ Brown Lake } & \multicolumn{3}{|c|}{ Peary Lake } & \multicolumn{3}{|c|}{ Ryan Lake } & \multicolumn{3}{|c|}{ Shoepack Lake } \\
\hline & & $\begin{array}{c}\text { Total } \\
\text { length } \\
(\mathrm{mm})\end{array}$ & $\begin{array}{c}\text { Wet } \\
\text { weight } \\
\text { (g) }\end{array}$ & $\begin{array}{c}\text { Total Hg } \\
\text { (ng/g wet } \\
\text { weight) }\end{array}$ & $\begin{array}{l}\text { Total } \\
\text { length } \\
\text { (mm) }\end{array}$ & $\begin{array}{c}\text { Wet } \\
\text { weight } \\
\text { (g) }\end{array}$ & $\begin{array}{l}\text { Total Hg } \\
\text { (ng/g wet } \\
\text { weight) }\end{array}$ & $\begin{array}{l}\text { Total } \\
\text { length } \\
(\mathrm{mm})\end{array}$ & $\begin{array}{c}\text { Wet } \\
\text { weight } \\
\text { (g) }\end{array}$ & $\begin{array}{c}\text { Total Hg } \\
\text { (ng/g wet } \\
\text { weight) }\end{array}$ & $\begin{array}{c}\text { Total } \\
\text { length } \\
(\mathrm{mm})\end{array}$ & $\begin{array}{c}\text { Wet } \\
\text { weight } \\
\text { (g) }\end{array}$ & $\begin{array}{c}\text { Total Hg } \\
\text { (ng/g wet } \\
\text { weight) }\end{array}$ \\
\hline \multirow[t]{3}{*}{2000} & Mean & 48 & 1.07 & 97 & 64 & 2.20 & 94 & 47 & 0.91 & 178 & 51 & 1.13 & 158 \\
\hline & SE & 0.42 & 0.02 & 2.0 & 3.1 & 0.32 & 2.8 & 0.37 & 0.02 & 3.7 & 0.92 & 0.07 & 6.2 \\
\hline & Min & 44 & 0.84 & 79 & 57 & 1.64 & 86 & 43 & 0.63 & 143 & 42 & 0.64 & 88 \\
\hline \multirow[t]{5}{*}{2001} & Mean & 51 & 1.11 & 59 & 47 & 0.88 & 67 & 62 & 2.20 & 177 & 53 & 1.30 & 119 \\
\hline & SE & 0.34 & 0.02 & 1.5 & 1.4 & 0.07 & 5.6 & 0.59 & 0.07 & 7.0 & 1.0 & 0.08 & 9.2 \\
\hline & Min & 48 & 0.96 & 46 & 40 & 0.56 & 50 & 58 & 1.73 & 135 & 50 & 1.15 & 85 \\
\hline & Max & 54 & 1.30 & 76 & 57 & 1.35 & 111 & 66 & 2.87 & 247 & 56 & 1.59 & 142 \\
\hline & $n$ & 20 & 20 & 20 & 10 & 10 & 10 & 19 & 19 & 19 & 5 & 5 & 5 \\
\hline 2003 & $n$ & 47 & 47 & 47 & 30 & 30 & 30 & 29 & 29 & 29 & & & \\
\hline \multirow[t]{5}{*}{2004} & Mean & 50 & 1.08 & 57 & 58 & 1.83 & 31 & 66 & 2.37 & 180 & NA & NA & NA \\
\hline & SE & 0.48 & 0.03 & 1.6 & 0.28 & 0.04 & 0.65 & 0.71 & 0.06 & 4.71 & & & \\
\hline & Min & 43 & 0.75 & 42 & 56 & 1.49 & 25 & 60 & 1.71 & 140 & & & \\
\hline & Max & 55 & 1.41 & 81 & 60 & 2.22 & 35 & 79 & 3.48 & 258 & & & \\
\hline & $n$ & 30 & 30 & 30 & 23 & 23 & 23 & 29 & 29 & 29 & & & \\
\hline \multirow[t]{4}{*}{2005} & Mean & 53 & 1.34 & 73 & 55 & 1.35 & 92 & 50 & 1.09 & 89 & 60 & 1.68 & 111 \\
\hline & SE & 0.67 & 0.05 & 1.8 & 0.61 & 0.05 & 14 & 1.4 & 0.09 & 14.3 & 0.49 & 0.05 & 6.68 \\
\hline & Min & 46 & 0.91 & 57 & 51 & 1.08 & 35 & 43 & 0.73 & 35 & 55 & 1.16 & 47 \\
\hline & Max & 30 & 2.04 & 105 & 58 & 1.65 & 167 & 64 & 2.06 & 174 & 64 & 2.09 & 159 \\
\hline
\end{tabular}

Table 7. Analysis of variance used to test for differences in mercury concentrations and total length of age-1 yellow perch for the three control lakes and Shoepack Lake before and after the Section 33 wildfire of 2004.

[The effect of the wildfire (Impact) was tested by first determining if the Time(After fire) $\times$ Control interactions were significant and then determining if the Time(After fire) $\times$ Impact interactions were significant. df, degrees of freedom; MS, mean square; F, distribution of two ratios; and P, probability value; error term, denominator for the calculation of the F statistic. See Underwood (1992) for detailed explanation]

\begin{tabular}{|c|c|c|c|c|c|c|c|}
\hline \multirow{2}{*}{ Source of variation } & \multirow{2}{*}{ df } & \multicolumn{3}{|c|}{ Differences in mercury concentrations } & \multicolumn{3}{|c|}{ Differences in total length } \\
\hline & & MS & $\mathbf{F}$ & $\mathbf{P}$ & MS & $\mathbf{F}$ & $\mathbf{P}$ \\
\hline Time $($ After fire $) \times$ Among locations & 3 & 5,789 & - & - & 141 & - & - \\
\hline Time $($ After fire $) \times$ Among controls & 2 & 6,815 & $9.906^{2}$ & $<0.0001$ & 77 & $6.36^{2}$ & 0.002 \\
\hline
\end{tabular}

${ }^{1}$ The denominator for the $\mathrm{F}$ ratio was the mean square of Time(After) $\times$ Among controls.

${ }^{2}$ The denominator for the $\mathrm{F}$ ratio was the mean square of the residual. 


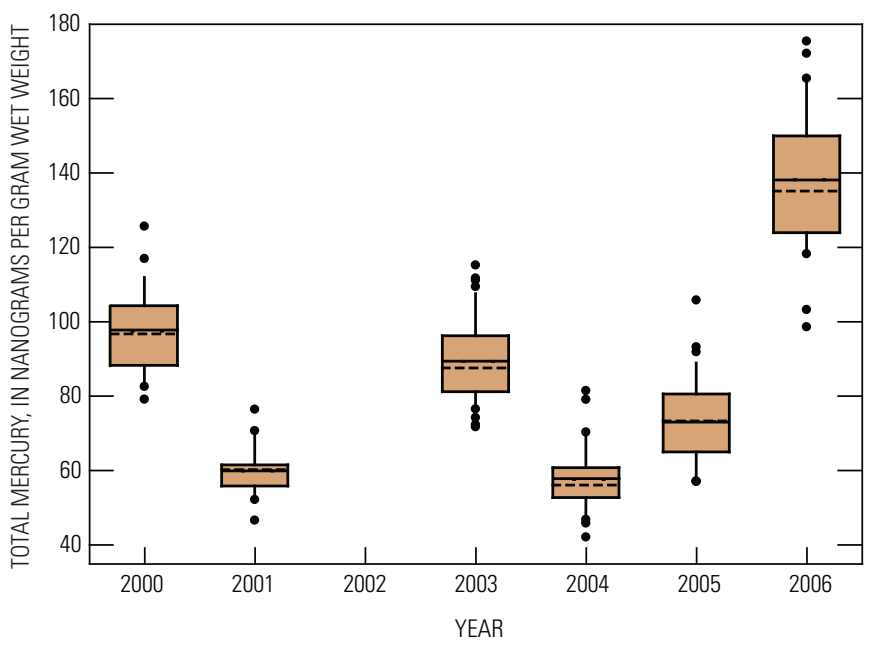

$A$

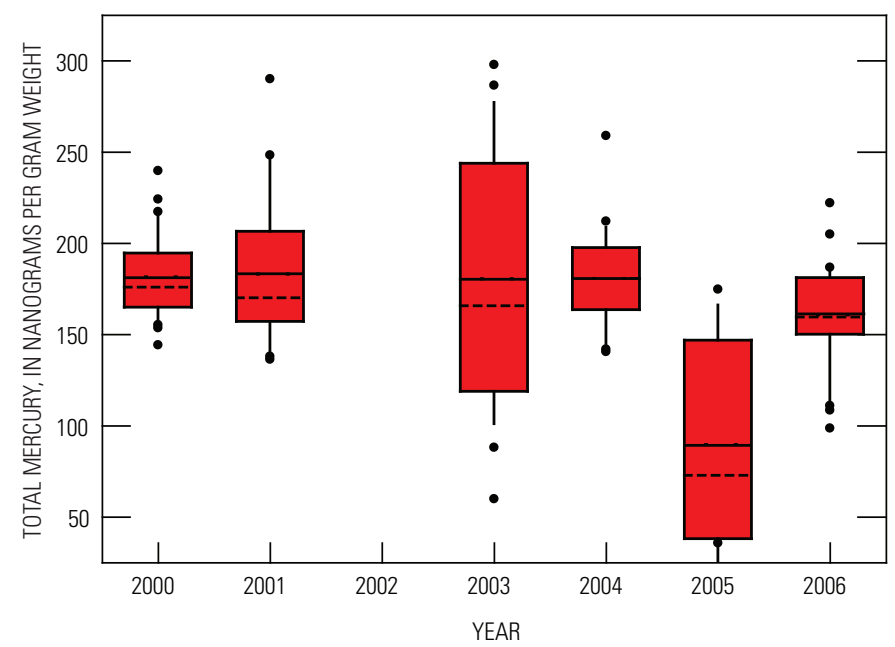

$B$

EXPLANATION

(n) Sample

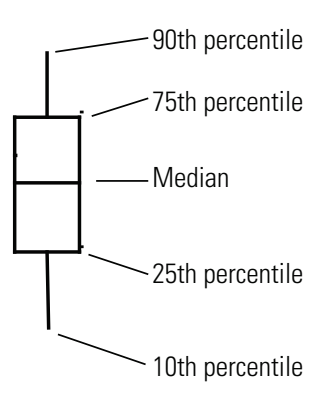

Figure 12. Box plots of total mercury concentrations in age-1 yellow perch from $A, B$, and $C$, the three control lakes-Brown, Peary, and Ryan-and $D$, fire-impacted Lake Shoepack. The mean is represented by the dotted line within the box, and individual outlying points are shown as solid circles.

chemistry. Comparison of soil chemistry between unburned sites selected within the Section 33 fire footprint and unburned control lakes confirms that site-to-site comparisons of soil chemistry across gradients of burn severity provide reliable data on the fate of mercury sequestered with organic carbon during fire. Mercury is mobilized from O-horizon soils by

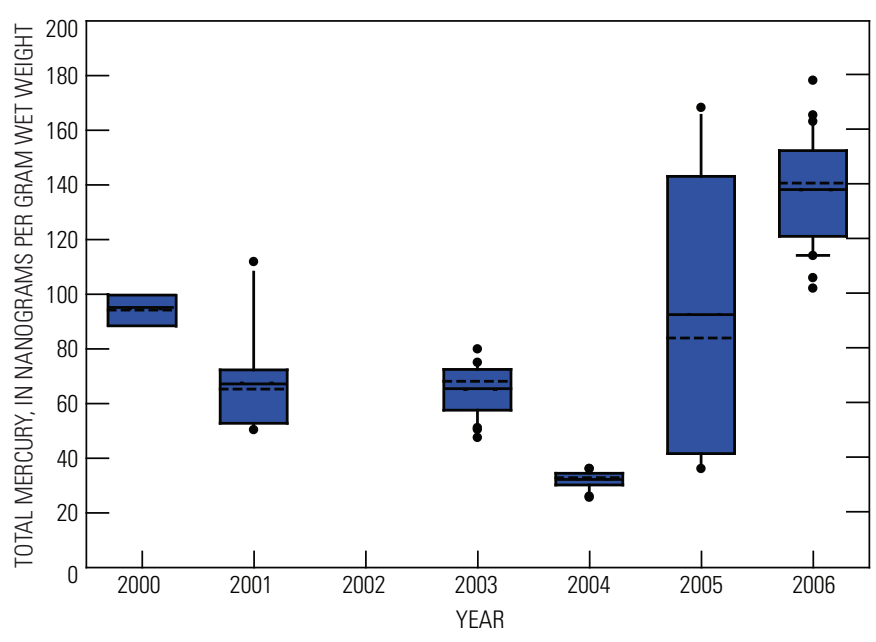

C

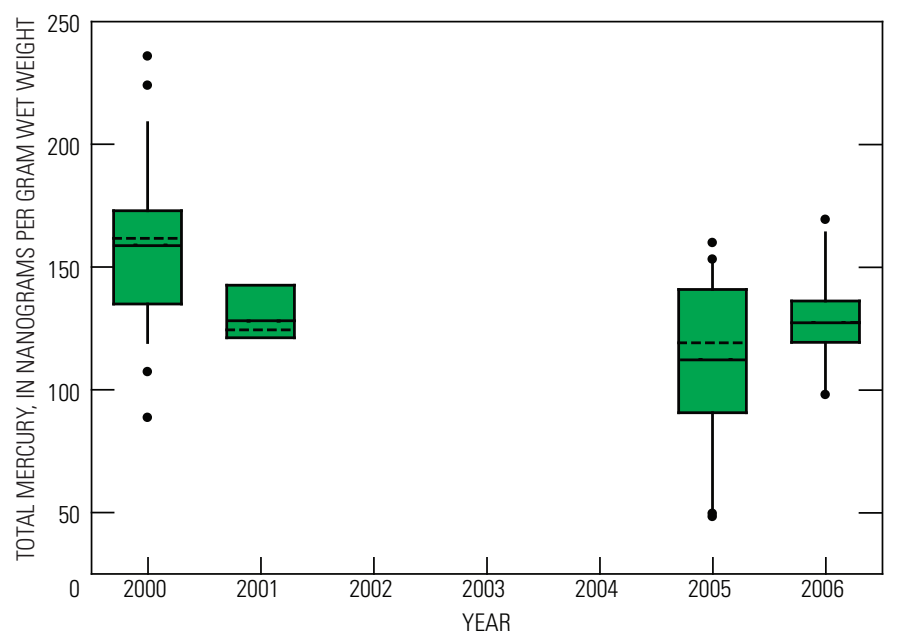

$D$

combustion of the forest floor, and also from A-horizon soils if the temperature and duration of fire results in high-fireseverity impacts to the forest floor. At high-severity sites in the Section 33 wildfire, $\sim 0.22 \mathrm{mg} \mathrm{Hg} / \mathrm{m}^{2}$ was mobilized by complete combustion of the $\mathrm{O}$ horizon, and $\sim 0.6$ to $0.9 \mathrm{mg} \mathrm{Hg} /$ $\mathrm{m}^{2}$ from the A horizon in these same areas. Mineral soils at low-moderate-severity sites show little significant changes in mercury values, although some mercury certainly is released by the partial combustion of organic soils.

Analyses of lake waters showed that despite the mobilization of significant quantities of mercury from watershed soils, aqueous total mercury and total organic carbon concentration in waters from Shoepack Lake were not significantly different from pre-fire concentrations. This lack of notable changes in lake-water chemistry following fire suggests that, in watersheds with relatively subdued topography, there may not be significant input of material from the burned landscape into lake waters. An overall downward trend in methylmercury concentrations in lake waters in three of the four lakes in 
VNP sampled in this study may be an indication of changes in regional mercury trends.

No changes in methylmercury for age-1 yellow perch, which provide a good baseline for a lake's aquatic food web, were found for perch in Shoepack Lake following the Section 33 wildfire. Statistical analyses indicate that this lack of change in methylmercury concentrations was the result of a lack of effect of fire on the bioavailability of methylmercury in the lake rather than on growth dilution.

In the timeframe of this study, we conclude that fire can mobilize large quantities of the mercury that have accumulated in forest soils over time. However, in forested watersheds with relatively subdued topography, similar to those in VNP, the bulk of this mercury apparently does not make its way into either a burned watershed's lake or aquatic food web.

\section{References Cited}

Allen, E.A., Prepas, E.E., Gabos, Stephan, Strachan, W.M.J., and Zhang, Weiping, 2005, Methyl mercury concentrations in macroinvertebrates and fish from burned and undisturbed lakes on the Boreal Plain: Canadian Journal of Fisheries and Aquatic Science, v. 62, p. 1963-1977.

American Public Health Association, American Water Works Association, and Water Environment Federation, 1998, Standard methods for the examination of water and wastewater (20th ed.): Baltimore, Md., United Book Press, Inc., variously paged.

Amirbahman, Aria, Ruck, P.L., Fernandez, I.J., Haines, T.A., and Kahl, J.S., 2004, The effect of fire on mercury cycling in the soils of forested watersheds: Acadia National Park, Maine, U.S.A.: Water, Air, \& Soil Pollution, v. 152, p. $315-331$.

Biswas, Abir, Blum, J.D., Klaue, Bjoern, and Keeler, G.J., 2007, Release of mercury from Rocky Mountain forest fires: Global Biogeochemical Cycles, v. 21, GB1002. (Also available at $h t t p: / / d x$.doi.org/10.1029/2006GB002696.)

Caldwell, C.A., Canavan, C.M., and Bloom, N.S., 2000, Potential effects of forest fire and storm flow on total mercury and methylmercury in sediments of an arid-lands reservoir: Science of the Total Environment, v. 260, p. 125-133.

Day, W.C., Southwick, D.L., Schulz, K.J., and Klein, T.L., 1990, Bedrock geologic map of the International Falls $1^{\circ}$ $\times 2^{\circ}$ quadrangle, Minnesota, United States and Ontario, Canada: U.S. Geological Survey Miscellaneous Investigations Map I-1965-B, scale 1:250,000.

DeVries, D.R., and Frie, R.V., 1996, Determination of age and growth, in Murphy, B.R., and Willis, D.W. (eds.), Fisheries Techniques ( $2 \mathrm{~d}$ ed.): Bethesda, Md., American Fisheries Society, p. 483-512.
DeWild, J.F., Olson, M.L., and Olund, S.D., 2002, Determination of methyl mercury by aqueous phase ethylation, followed by gas chromatographic separation with cold vapor atomic fluorescence detection: U.S. Geological Survey Open-File Report 01-445, 14 p. (Also available at http://pubs.usgs.gov/of/2001/ofr-01-445/.)

Engle, M.A., Gustin, M.S., Johnson, D.W., Murphy, J.F., Miller, W.W., Walker, R.F., Wright, Joan, and Markee, Melissa, 2005, Mercury distribution in two Sierran forest and one desert sagebrush steppe ecosystems and the effects of fire: Science of the Total Environment, v. 367, p. 222-233.

Engstrom, D.R., and Swain, E.B., 1997, Recent declines in atmospheric mercury deposition in the upper Midwest: Environmental Science and Technology, v. 31, p. 960-967.

Engstrom, D.R., Balogh, S.J., and Swain, E.B., 2007, History of mercury inputs to Minnesota lakes: Influence of watershed disturbance and localized atmospheric deposition: Limnology and Oceanography, v. 52, p. 2467-2483.

Erickson, R., 1981, Minnesota Soil Atlas, International FallsTwo Harbors sheets: University of Minnesota Agricultural Experiment Station Miscellaneous Report 177, 2 maps, scale 1:250,000.

Fishman, M.J., and Friedman, L.C., eds., 1989, Methods for the determination of inorganic substances in water and fluvial sediments: U.S. Geological Survey Techniques of Water-Resources Investigations, book 5, chap. A1, 545 p.

Fitzgerald, W.F., Engstrom, D.R., Mason, R.P., and Nater, E.A., 1998, The case for atmospheric mercury contamination in remote areas: Environmental Science and Technology, v. 32, p. 1-7.

Garcia, Edinese and Carignan, Richard, 1999, Impact of wildfire and clear-cutting in the boreal forest on methyl mercury in zooplankton: Canadian Journal of Fisheries and Aquatic Science, v. 56, p. 339-345.

Garcia, Edenise and Carignan, Richard, 2000, Mercury concentrations in northern pike (Esox lucius) from boreal lakes with logged, burned, or undisturbed catchments: Canadian Journal of Fisheries and Aquatic Science, v. 57 (supplement 2), p. 129-135.

Garcia, Edenise and Carignan, Richard, 2005, Mercury concentrations in fish from forest harvesting and fire-impacted Canadian boreal lakes compared using stable isotopes of nitrogen: Environmental Toxicology and Chemistry, v. 24, p. 685-693.

Goldstein, R.M., Brigham, M.E., Stuewe, Luke, and Menheer, M.A., 2003, Mercury data from small lakes in Voyageurs National Park, northern Minnesota, 2000-02: U.S. Geologi- 
cal Survey Open-File Report 03-480, 18 p. (Also available at http://pubs.usgs.gov/of/2003/ofro3-480/.)

Grigal, D.F., 2002, Inputs and outputs of mercury from terrestrial watersheds: a review: Environmental Review, v. 10, p. 1-39.

Harden, J.W., Neff, J.C., Sandberg, D.V., Turetsky, M.R., Ottmar, R.D., Gleixner, Gerd, Fries, T.L., and Maines, K.L., 2004, Chemistry of burning the forest floor during the FROSTFIRE experimental burn, interior Alaska, 1999: Global Biogeochemical Cycles, v. 18, GB3014. (Also available at http://dx.doi.org/10.1029/2003GB002194.)

Heinselman, M.L., 1996, The Boundary Waters Wilderness Ecosystem: University of Minnesota Press, Minneapolis, Minn., 334 p.

Kelly, E.N., Schindler, D.W., St. Louis, V.L., Donald, D.B., and Vladicka, K.E., 2006, Forest fire increases mercury accumulation by fishes via food web restructuring and increased mercury inputs: Proceedings of the National Academy of Science. (Also available at http://dx.doi.org/10.1073/pnas.0609798104.)

Krabbenhoft, D.P., and Fink, L.E., 2000, The effect of dry down and natural fires on mercury methylation in the Florida Everglades, in Nriagu, J., ed., 11th Annual International Conference on Heavy Metals in the Environment, Contribution \#1044: University of Michigan, School of Public Health, Ann Arbor, Mich.

Nater, E.A., and Grigal, D.F., 1992, Regional trends in mercury distributions across the Great Lakes states, north central USA: Nature, v. 358, p. 139-141. (Also available at http://dx.doi.org/10.1038/358139a0.)

Nelson, S.J., Johnson, K.B., Kahl, J.S., Haines, T.A., and Fernandez, I.J., 2007, Mass balances of mercury and nitrogen in burned and unburned forested watersheds at Acadia National Park, Maine, USA: Environmental Monitoring and Assessment, v. 126, p. 69-80.

Olson, M.L., and DeWild, J.F., 1999, Techniques for the collection and species-specific analysis of low levels of mercury in water, sediment, and biota, in Morganwalp, D.W., and Buxton, H.T., eds., U.S. Geological Survey Toxic Substances Hydrology Program-Proceedings of the technical meeting, Charleston, S.C., March 8-12, 1999, v. 2 of 3, Contamination of hydrologic systems and related ecosystems: U.S. Geological Survey Water-Resources Investigations Report 99-4018-B, p. 191-200, accessed August 5, 2008, at http://toxics.usgs.gov/pubs/wri99-4018/Volume2/.

Swain, E.B., Engstrom, D.R., Brigham, M.E., Henning, T.A., and Brezonik, P.L., 1992, Increasing rates of atmospheric mercury deposition in midcontinental North America: Science, v. 257, p. 784-787.

Taggert, J.E., Jr., ed., 2002, Analytical methods for chemical analysis of geologic and other material, U.S. Geological Survey: U.S. Geological Survey Open-File Report 02-0223, 20 p. (Also available at http://pubs.usgs.gov/of/2002/ ofr-02-0223/OFR-02-0223.pdf.)

Turetsky, M.R., Harden, J.W., Friedli, H.R., Flannigan, Mike, Payne, Nicholas, Crock, James, and Radke, Lawrence, 2006, Wildfires threaten mercury stocks in northern soils: Geophysical Research Letters, 33, L16403. (Also available at http://dx.doi.org/10.1029/2005GL025595.)

Underwood, A. J., 1992, Beyond BACI: The detection of environmental impacts on populations in the real, but variable, world: Journal of Experimental Marine Biology and Ecology, v. 161, p. 145-178.

Underwood, A. J., 1994, On beyond BACI: Sampling designs that might reliably detect environmental disturbances: Ecological Applications, v. 4, p. 3-15.

U.S. Environmental Protection Agency, 2001, Appendix to method 1631-Total mercury in tissue, sludge, sediment, and soil by acid digestion and $\mathrm{BrCl}$ oxidation: U.S. Environmental Protection Agency Report EPA 821-R-01-013.

U.S. Environmental Protection Agency, 2002, Method 1631, revision E-Mercury in water by oxidation, purge and trap, and cold vapor atomic fluorescence spectrometry: U.S. Environmental Protection Agency Report EPA-821-R-02-019, 38 p., accessed August 5, 2008, at http://www.epa.gov/waterscience/methods/1631e.pdf.

Wiener, J.G., Bodaly, R.A., Brown, S.S., Lucotte, Marc, Newman, M.C., Porcella, D.B., Reash, R.J., and Swain, E.B., 2007, Monitoring and evaluating trends in methylmercury accumulation in aquatic biota, in Harris, R. C., Krabbenhoft and others, eds., Ecosystem Responses to Mercury Contamination: Indicators of Change: Boca Raton, Fla., CRC Press/ Taylor and Francis, p. 87-122.

Wiener, J.G., Knights, B.C., Sandheinrich, M.B., Jeremiason, J.D., Brigham, M.E., Engstrom, D.R., Woodruff, L.G., Cannon, W.F., and Balogh, S.J., 2006, Mercury in soils, lakes, and fish in Voyageurs National Park (Minnesota): Importance of atmospheric deposition and ecosystem factors: Environmental Science and Technology, v. 40, p. $6261-6268$.

Woodruff, L.G., and Cannon, W.F., 2002, The effect of fire on mercury and carbon in forest soils: results from northern Michigan and Minnesota: Geological Society of America Program with Abstracts, v. 33, p. 186. 
Click back to Contents

Appendix 1-A Horizon Chemistry, Ryan and Brown Lakes, Voyageurs National Park, MN

\begin{tabular}{|c|c|c|c|c|c|c|c|c|c|c|c|c|c|c|c|c|c|c|c|c|c|c|c|c|c|c|c|c|}
\hline Field no. & $\begin{array}{c}\text { Year } \\
\text { collected }\end{array}$ & Latitude & Longitude & Watershed & $\begin{array}{c}\mathrm{Al} \\
\text { (wt. \%) }\end{array}$ & $\begin{array}{c}\mathrm{Ca} \\
\text { (wt. \%) }\end{array}$ & \begin{tabular}{|c}
$\mathrm{Fe}$ \\
(wt. \%)
\end{tabular} & $\begin{array}{c}\mathrm{K} \\
\text { (wt. \%) }\end{array}$ & $\begin{array}{c}\mathrm{Mg} \\
\text { (wt. \%) }\end{array}$ & \begin{tabular}{|c}
$\mathrm{Na}$ \\
(wt. \%)
\end{tabular} & $\begin{array}{c}\mathrm{S} \\
\text { (wt. \%) }\end{array}$ & $\begin{array}{c}\mathrm{Ti} \\
\text { (wt. \%) }\end{array}$ & \begin{tabular}{|c|}
$\mathrm{Ag}$ \\
$(\mathrm{ppm})$
\end{tabular} & $\begin{array}{c}\text { As } \\
(\mathrm{ppm})\end{array}$ & \begin{tabular}{|c|}
$\mathrm{Ba}$ \\
$(\mathrm{ppm})$ \\
\end{tabular} & $\begin{array}{c}\mathrm{Be} \\
(\mathrm{ppm})\end{array}$ & $\begin{array}{c}\mathrm{Bi} \\
(\mathrm{ppm})\end{array}$ & $\begin{array}{c}\mathrm{Cd} \\
(\mathrm{ppm})\end{array}$ & $\begin{array}{c}\mathrm{Ce} \\
(\mathrm{ppm})\end{array}$ & $\begin{array}{c}\begin{array}{c}\text { Co } \\
(\mathrm{ppm})\end{array} \\
\end{array}$ & $\begin{array}{c}\mathrm{Cr} \\
(\mathrm{ppm})\end{array}$ & $\begin{array}{c}\mathrm{Cs}^{*} \\
(\mathrm{ppm})\end{array}$ & $\begin{array}{c}\mathrm{Cu} \\
(\mathrm{ppm})\end{array}$ & $\begin{array}{c}\mathrm{Ga} \\
(\mathrm{ppm})\end{array}$ & \begin{tabular}{c|}
$\mathrm{Hg}$ \\
$(\mathrm{ppm})$
\end{tabular} & $\begin{array}{c}\text { In } \\
\text { (ppm) }\end{array}$ & $\begin{array}{c}\mathrm{La} \\
(\mathrm{ppm})\end{array}$ & $\begin{array}{c}\mathrm{Li} \\
\text { (ppm) }\end{array}$ \\
\hline $\begin{array}{l}\mathrm{V}-03-01 \mathrm{~A} \\
\end{array}$ & 2003 & 48.51360 & -92.70532 & Ryan Lake & 4.30 & 1.03 & \begin{tabular}{|l|}
1.85 \\
\end{tabular} & 1.00 & 0.42 & \begin{tabular}{|l|}
0.96 \\
\end{tabular} & 0.07 & 0.18 & \begin{tabular}{|l|}
$<1$ \\
\end{tabular} & 7 & \begin{tabular}{|l|}
578 \\
\end{tabular} & 1.0 & 0.35 & 0.5 & 40.0 & 12.2 & 32 & 3.08 & 14.7 & 10.4 & 0.15 & 0.05 & 20.2 & 18 \\
\hline V-03-02A & 2003 & 48.52164 & -92.70490 & Ryan Lake & 2.14 & 0.76 & 1.00 & 0.43 & 0.25 & 0.26 & 0.12 & 0.08 & $<1$ & 3 & 345 & 1.1 & 0.12 & 0.4 & 99.4 & 25.8 & 19 & 1.99 & 17.8 & 4.8 & 0.15 & 0.02 & 43.7 & 10 \\
\hline V-03-03A & 2003 & 48.52124 & -92.70567 & Ryan Lake & 3.58 & 0.81 & 0.99 & 0.96 & 0.26 & 1.00 & 0.07 & 0.13 & $<1$ & 3 & 368 & 0.8 & 0.14 & 0.1 & 41.4 & 8.8 & 22 & 1.97 & 15.2 & 7.9 & 0.12 & 0.03 & 24.2 & 8 \\
\hline V-03-04A & 2003 & 48.51987 & -92.70518 & Ryan Lake & 3.78 & 0.63 & 2.13 & 0.74 & 0.33 & 0.62 & 0.08 & 0.16 & $<1$ & 6 & 414 & 1.1 & 0.28 & 0.3 & 58.3 & 37.3 & 37 & 2.30 & 41.4 & 9.3 & 0.17 & 0.04 & 27.7 & 14 \\
\hline V-03-05A & 2003 & 48.51889 & -92.70403 & Ryan Lake & 3.24 & 1.31 & 1.17 & 0.92 & 0.29 & 0.80 & 0.08 & 0.14 & $<1$ & 5 & 524 & 0.7 & 0.30 & 0.6 & 19.1 & 7.5 & 22 & 1.86 & 10.4 & 7.4 & 0.18 & 0.04 & 10.3 & 9 \\
\hline V-03-05A dup & 2003 & 48.51889 & -92.70403 & Ryan Lake & 3.18 & 1.28 & 1.12 & 0.92 & 0.29 & 0.77 & 0.08 & 0.14 & $<1$ & 5 & 508 & 0.7 & 0.30 & 0.6 & 19.8 & 7.4 & 24 & 2.03 & 11.3 & 7.3 & 0.17 & 0.03 & 10.7 & 9 \\
\hline V-03-06A & 2003 & 48.51519 & -92.70383 & Ryan Lake & 3.43 & 1.07 & 1.75 & 0.70 & 0.35 & 0.57 & 0.09 & 0.16 & $<1$ & 7 & 554 & 0.7 & 0.31 & 0.9 & 22.2 & 9.3 & 37 & 3.50 & 16.4 & 8.3 & 0.21 & 0.03 & 11.7 & 16 \\
\hline V-03-07A & 2003 & 48.51681 & -92.70174 & Ryan Lake & 3.14 & 1.32 & 1.80 & 0.59 & 0.37 & 0.65 & 0.09 & 0.14 & $<1$ & 6 & 359 & 0.7 & 0.23 & 0.5 & 15.5 & 8.6 & 77 & 2.90 & 32.5 & 7.3 & 0.18 & 0.03 & 8.5 & 12 \\
\hline V-03-08A & 2003 & 48.51711 & -92.70057 & Ryan Lake & 2.06 & 0.58 & 0.83 & 0.47 & 0.18 & 0.48 & 0.09 & 0.08 & $<1$ & 4 & 334 & 0.4 & 0.19 & 0.2 & 11.8 & 4.8 & 17 & 0.86 & 8.6 & 4.3 & 0.20 & $<0.02$ & 6.9 & 5 \\
\hline V-03-09A & 2003 & 48.51831 & -92.70119 & Ryan Lake & 3.19 & 1.37 & 1.31 & 0.80 & 0.33 & 0.74 & 0.08 & 0.14 & $<1$ & 4 & 707 & 0.7 & 0.24 & 0.9 & 18.0 & 7.5 & 25 & 1.91 & 9.1 & 7.1 & 0.17 & 0.03 & 9.3 & 10 \\
\hline V-03-10A & 2003 & 48.51792 & -92.69938 & Ryan Lake & 2.77 & 0.87 & 0.79 & 0.74 & 0.24 & 0.74 & 0.08 & 0.10 & $<1$ & 4 & 427 & 0.6 & 0.20 & 0.3 & 15.1 & 9.2 & 16 & 1.09 & 9.4 & 5.8 & 0.19 & 0.02 & 8.4 & 6 \\
\hline V-03-11A & 2003 & 48.51568 & -92.70500 & Ryan Lake & 3.51 & 0.96 & 1.13 & 0.98 & 0.27 & 0.96 & 0.06 & 0.13 & $<1$ & 3 & 476 & 0.7 & 0.18 & 0.3 & 17.3 & 7.4 & 22 & 1.79 & 10.1 & 7.8 & 0.12 & 0.02 & 9.4 & 9 \\
\hline V-03-11A dup & 2003 & 48.51568 & -92.70500 & Ryan Lake & 3.59 & 0.99 & 1.17 & 1.04 & 0.29 & 0.97 & 0.06 & 0.14 & $<1$ & 4 & 499 & 0.8 & 0.20 & 0.3 & 18.1 & 8.2 & 25 & 1.59 & 12.1 & 8.2 & 0.12 & 0.03 & 9.8 & 10 \\
\hline V-03-12A & 2003 & 48.51580 & -92.70547 & Ryan Lake & 5.40 & 1.00 & 1.70 & 1.30 & 0.43 & 1.58 & 0.03 & 0.20 & $<1$ & 3 & 408 & 1.0 & 0.22 & $<0.1$ & 17.4 & 6.6 & 43 & 1.43 & 12.0 & 11.5 & 0.06 & 0.03 & 9.6 & 17 \\
\hline V-03-13A & 2003 & 48.51373 & -92.70505 & Ryan Lake & 3.68 & 0.94 & 1.65 & 0.85 & 0.39 & 0.92 & 0.07 & 0.17 & $<1$ & 6 & 377 & 0.8 & 0.26 & 0.2 & 17.6 & 7.0 & 35 & 2.63 & 10.0 & 8.9 & 0.15 & 0.03 & 9.1 & 14 \\
\hline V-03-14A & 2003 & 48.51351 & -92.70242 & Ryan Lake & 3.65 & 1.19 & 1.13 & 1.05 & 0.32 & 1.03 & 0.07 & 0.14 & $<1$ & 3 & 536 & 0.8 & 0.24 & 0.5 & 21.9 & 15.6 & 24 & 1.51 & 12.9 & 7.9 & 0.11 & 0.03 & 13.1 & 8 \\
\hline V-03-15A & 2003 & 48.51373 & -92.78778 & Brown Lake & 4.88 & 1.34 & 1.18 & 1.51 & 0.33 & 1.64 & 0.05 & 0.13 & $<1$ & 3 & 544 & 0.9 & 0.23 & 0.4 & 17.6 & 7.3 & 25 & 2.18 & 13.0 & 9.8 & 0.08 & 0.03 & 9.0 & 11 \\
\hline V-03-16A & 2003 & 48.51392 & -92.78711 & Brown Lake & 3.77 & 1.08 & 1.62 & 1.06 & 0.39 & 0.81 & 0.08 & 0.17 & $<1$ & 6 & 589 & 0.8 & 0.35 & 0.5 & 23.6 & 14.5 & 32 & 2.52 & 15.2 & 9.6 & 0.14 & 0.04 & 13.2 & 16 \\
\hline V-03-17A & 2003 & 48.51284 & -92.78577 & Brown Lake & 4.45 & 0.76 & 2.04 & 1.00 & 0.43 & 0.76 & 0.09 & 0.23 & $<1$ & 4 & 572 & 1.4 & 0.32 & 0.4 & 71.5 & 54.7 & 43 & 3.61 & 29.7 & 11.0 & 0.13 & 0.04 & 29.3 & 23 \\
\hline V-03-18A & 2003 & 48.51218 & -92.78512 & Brown Lake & 3.44 & 1.02 & 1.70 & 0.59 & 0.42 & 0.76 & 0.10 & 0.17 & $<1$ & 4 & 487 & 0.9 & 0.31 & 0.9 & 18.6 & 12.3 & 46 & 3.67 & 25.5 & 8.5 & 0.12 & 0.03 & 10.2 & 16 \\
\hline V-06-01A & 2006 & 48.51472 & -92.70396 & Ryan Lake & 4.23 & 0.79 & 1.78 & 0.72 & 0.31 & 0.84 & 0.05 & 0.19 & $<1$ & 7 & 411 & 1.2 & 0.24 & 0.5 & 21.9 & 7.6 & 37 & $<5$ & 16.4 & 10.2 & 0.17 & 0.03 & 12.1 & 14 \\
\hline V-06-02A & 2006 & 48.51580 & -92.70547 & Ryan Lake & 3.18 & 0.78 & 1.01 & 0.76 & 0.25 & 0.77 & 0.06 & 0.12 & $<1$ & 5 & 322 & 0.8 & 0.19 & 0.3 & 17.2 & 4.6 & 24 & $<5$ & 12.5 & 7.6 & 0.20 & 0.02 & 9.9 & 7 \\
\hline V-06-03A & 2006 & 48.51574 & -92.70515 & Ryan Lake & 5.10 & 0.97 & 2.02 & 1.01 & 0.43 & 1.26 & 0.04 & 0.24 & $<1$ & 5 & 444 & 1.1 & 0.25 & 0.4 & 26.1 & 13.2 & 48 & $<5$ & 21.7 & 13.6 & 0.08 & 0.03 & 14.5 & 16 \\
\hline V-06-04A & 2006 & 48.51947 & -92.70412 & Ryan Lake & 3.31 & 1.35 & 1.06 & 0.97 & 0.33 & 0.81 & 0.09 & 0.12 & $<1$ & 6 & 396 & 0.8 & 0.16 & 0.6 & 14.4 & 5.6 & 25 & $<5$ & 8.6 & 7.6 & 0.14 & 0.03 & 7.8 & 8 \\
\hline V-06-05A & 2006 & 48.52139 & -92.70499 & Ryan Lake & 4.86 & 0.77 & 2.13 & 1.13 & 0.47 & 0.80 & 0.07 & 0.19 & $<1$ & 7 & 524 & 1.6 & 0.18 & 0.4 & 80.2 & 57.7 & 46 & $<5$ & 25.5 & 11.7 & 0.10 & 0.04 & 26.2 & 22 \\
\hline V-06-06A & 2006 & 48.52126 & -92.70521 & Ryan Lake & 4.47 & 0.96 & 0.79 & 1.33 & 0.23 & 1.40 & 0.04 & 0.12 & $<1$ & 3 & 429 & 0.9 & 0.07 & 0.2 & 20.6 & 4.6 & 21 & $<5$ & 13.4 & 9.8 & 0.07 & 0.02 & 11.7 & 5 \\
\hline V-06-07A & 2006 & 48.52016 & -92.70523 & Ryan Lake & 4.82 & 1.00 & 1.54 & 1.27 & 0.37 & 1.20 & 0.05 & 0.16 & $<1$ & 6 & 465 & 0.9 & 0.12 & 0.2 & 17.1 & 13.5 & 34 & $<5$ & 12.9 & 11.3 & 0.13 & 0.02 & 9.8 & 11 \\
\hline V-06-08A & 2006 & 48.51816 & -92.70107 & Ryan Lake & 4.88 & 1.32 & 1.71 & 1.28 & 0.41 & 1.3 & 0.05 & 0.20 & $<1$ & 4 & 604 & 0.9 & 0.14 & 0.5 & 17.6 & 7.7 & 43 & $<5$ & 9.3 & 11.8 & 0.09 & 0.03 & 9.8 & 12 \\
\hline V-06-09A & 2006 & 48.51760 & -92.70024 & Ryan Lake & 2.71 & 0.76 & 0.77 & 0.68 & 0.23 & 0.73 & 0.07 & 0.10 & $<1$ & 3 & 362 & 0.7 & 0.09 & 0.4 & 12.8 & 5.4 & 22 & $<5$ & 9.3 & 6.2 & 0.15 & 0.02 & 7.7 & 4 \\
\hline V-06-10A & 2006 & 48.51627 & -92.70162 & Ryan Lake & 3.36 & 1.57 & 1.41 & 0.66 & 0.41 & 0.75 & 0.10 & 0.15 & $<1$ & 5 & 432 & 0.7 & 0.20 & 0.7 & 15.3 & 10.7 & 41 & $<5$ & 10.2 & 8.1 & 0.16 & 0.03 & 8.7 & 11 \\
\hline V-06-11A & 2006 & 48.51359 & -92.70248 & Ryan Lake & 3.55 & 1.18 & 0.92 & 1.11 & 0.30 & 0.99 & 0.07 & 0.12 & $<1$ & 2 & 454 & 0.8 & 0.08 & 0.5 & 14.3 & 10.4 & 28 & $<5$ & 9.5 & 8.0 & 0.09 & 0.02 & 8.6 & 6 \\
\hline V-06-12A & 2006 & 48.71781 & -92.69928 & Ryan Lake & 2.72 & 0.78 & 0.71 & 0.75 & 0.22 & 0.69 & 0.09 & 0.10 & $<1$ & 4 & 382 & 0.6 & 0.08 & 0.3 & 13.4 & 6.4 & 22 & $<5$ & 10.8 & 6.4 & 0.15 & 0.02 & 7.6 & 6 \\
\hline V-06-13A & 2006 & 48.51214 & -92.78509 & Brown Lake & 4.94 & 1.32 & 2.38 & 0.75 & 0.61 & 1.25 & 0.06 & 0.24 & $<1$ & 5 & 483 & 1.1 & 0.19 & 1.0 & 17.2 & 14.6 & 72 & $<5$ & 22.2 & 12.7 & 0.07 & 0.03 & 9.8 & 22 \\
\hline V-06-14A & 2006 & 48.51289 & -92.78571 & Brown Lake & 4.56 & 0.71 & 1.84 & 0.92 & 0.41 & 0.63 & 0.10 & 0.18 & $<1$ & 5 & 474 & 1.9 & 0.22 & 0.4 & 114.0 & 87.6 & 45 & $<5$ & 42.9 & 9.3 & 0.13 & 0.04 & 68.5 & 20 \\
\hline V-06-15A & 2006 & 48.51385 & -92.78703 & Brown Lake & 5.44 & 1.17 & 1.20 & 1.71 & 0.38 & 1.87 & 0.03 & 0.14 & $<1$ & 3 & 458 & 1.1 & 0.10 & 0.2 & 16.1 & 7.3 & 32 & $<5$ & 4.3 & 12.1 & 0.04 & 0.02 & 8.7 & 12 \\
\hline V-06-15A dup & 2006 & 48.51383 & -92.78757 & Brown Lake & 5.49 & 1.20 & 1.15 & 1.72 & 0.37 & 1.88 & 0.03 & 0.14 & $<1$ & 3 & 459 & 1.3 & 0.09 & 0.2 & 17.9 & 8.5 & 36 & $<5$ & 5.1 & 11.7 & 0.04 & 0.02 & 9.7 & 12 \\
\hline V-06-16A & 2006 & 48.51383 & -92.78757 & Brown Lake & 4.10 & 1.39 & 1.47 & 1.20 & 0.42 & 0.96 & 0.06 & 0.16 & $<1$ & 4 & 572 & 1.1 & 0.24 & 0.8 & 22.0 & 11.8 & 35 & $<5$ & 15.7 & 9.4 & 0.10 & 0.03 & 11.9 & 14 \\
\hline
\end{tabular}


Appendix 1-A Horizon Chemistry, Ryan and Brown Lakes, Voyageurs National Park, MN

\begin{tabular}{|c|c|c|c|c|c|c|c|c|c|c|c|c|c|c|c|c|c|c|c|c|c|c|c|c|c|}
\hline Field no. & $\begin{array}{c}\text { Year } \\
\text { collected }\end{array}$ & Latitude & Longitude & Watershed & $\begin{array}{c}\mathrm{Mn} \\
(\mathrm{ppm})\end{array}$ & $\begin{array}{c}\text { Mo } \\
(\mathrm{ppm})\end{array}$ & $\begin{array}{c}\mathrm{Nb} \\
(\mathrm{ppm})\end{array}$ & $\begin{array}{c}\mathrm{Ni} \\
\text { (ppm) }\end{array}$ & $\begin{array}{c}\mathbf{P} \\
(\mathrm{ppm})\end{array}$ & $\begin{array}{c}\mathrm{Pb} \\
(\mathrm{ppm})\end{array}$ & $\begin{array}{c}\mathrm{Rb} \\
(\mathrm{ppm})\end{array}$ & $\begin{array}{c}\mathrm{Sb} \\
(\mathrm{ppm})\end{array}$ & $\begin{array}{c}\mathrm{Sc} \\
(\mathrm{ppm})\end{array}$ & $\begin{array}{c}\mathrm{Sn} \\
(\mathrm{ppm})\end{array}$ & $\begin{array}{c}\mathrm{Sr} \\
(\mathrm{ppm})\end{array}$ & $\begin{array}{c}\mathrm{Te} \\
(\mathrm{ppm})\end{array}$ & $\begin{array}{c}\text { Th } \\
(\mathrm{ppm})\end{array}$ & $\begin{array}{c}\mathrm{Tl} \\
(\mathrm{ppm})\end{array}$ & $\underset{(p p m)}{U}$ & $\begin{array}{c}V \\
(\mathrm{ppm})\end{array}$ & $\begin{array}{c}W \\
(\mathrm{ppm})\end{array}$ & $\begin{array}{c}\mathrm{Y} \\
(\mathrm{ppm})\end{array}$ & $\begin{array}{c}\mathrm{Zn} \\
(\mathrm{ppm})\end{array}$ & $\begin{array}{c}\mathrm{Se} \\
(\mathrm{ppm})\end{array}$ & $\begin{array}{l}\text { Total C } \\
\text { (wt. \%) }\end{array}$ \\
\hline V-03-01A & 2003 & 48.51360 & -92.70532 & Ryan Lake & 2,900 & 0.93 & 5.7 & 26.6 & 900 & 66.3 & 72.8 & 0.65 & \begin{tabular}{|l|}
6.1 \\
\end{tabular} & 1.6 & 168 & $<0.1$ & \begin{tabular}{|l|}
4.1 \\
\end{tabular} & 0.4 & 1.2 & 48 & \begin{tabular}{|l|l}
0.8 \\
\end{tabular} & 9.5 & 121 & 0.6 & 17.70 \\
\hline V-03-02A & 2003 & 48.52164 & 2.70490 & jan Lake & 2,400 & 0.60 & 2.6 & 26.8 & 1,140 & 21.8 & 38.6 & 0.42 & 4.8 & 0.5 & 93 & $<0.1$ & 4.5 & 0.2 & 1.6 & 26 & 0.4 & 13.7 & 53 & 0.6 & 33.10 \\
\hline V-03-03A & 2003 & 48.52124 & 2.70567 & an Lake & 333 & 0.54 & 3.9 & 12.8 & 620 & 28.6 & 59.3 & 0.49 & 4.7 & 1.1 & 159 & $<0.1$ & 3.0 & 0.2 & 1.6 & 31 & 0.4 & 8.7 & 32 & 0.4 & 20.20 \\
\hline V-03-04A & 2003 & 48.51987 & 92.70518 & Ryan Lake & 1,950 & 1.17 & 5.1 & 22.1 & 1,040 & 41.9 & 64.4 & 0.50 & 5.5 & 1.5 & 116 & $<0.1$ & 6.6 & 0.3 & 2.3 & 53 & 0.5 & 8.1 & 74 & 0.9 & 20.80 \\
\hline V-03-05A & 2003 & 48.51889 & -92.70403 & Ryan Lake & 2,620 & 0.65 & 4.3 & 12.6 & 750 & 61.3 & 65.7 & 0.65 & 4.2 & 1.4 & 151 & $<0.1$ & 3.2 & 0.3 & 0.9 & 34 & 0.5 & 5.1 & 109 & 0.5 & 22.50 \\
\hline V-03-05A dup & 2003 & 48.51889 & -92.70403 & Ryan Lake & 2,560 & 0.74 & 4.2 & 13.4 & 740 & 59.3 & 64.0 & 0.67 & 4.3 & 1.5 & 148 & $<0.1$ & 3.3 & 0.3 & 0.9 & 33 & 0.4 & 5.2 & 111 & 0.6 & 23.00 \\
\hline V-03-06A & 2003 & 48.51519 & 92.70383 & an Lake & 690 & 0.99 & 4.6 & 22.8 & 790 & 58.1 & 60.5 & 0.65 & 4.8 & 1.4 & 125 & $<0.1$ & 3.7 & 0.3 & 1.0 & 48 & 0.7 & 5.3 & 176 & 0.9 & 24.80 \\
\hline V-03-07A & 2003 & 48.51681 & 92.70174 & yan Lake & 1,970 & 15.30 & 4.2 & 30.1 & 630 & 49.2 & 48.9 & 0.72 & 3.9 & 2.2 & 131 & $<0.1$ & 3.0 & 0.3 & 0.8 & 41 & 1.3 & 3.9 & 159 & 0.8 & 28.20 \\
\hline V-03-08A & 2003 & 48.51711 & -92.70057 & Ryan Lake & 184 & 0.55 & 2.3 & 12.7 & 560 & 30.3 & 24.3 & 0.44 & 2.7 & 0.9 & 99 & $<0.1$ & 2.1 & 0.2 & 0.6 & 23 & 0.3 & 3.0 & 33 & 0.7 & 34.20 \\
\hline V-03-09A & 2003 & 48.51831 & -92.70119 & Ryan Lake & 2,740 & 0.53 & 4.0 & 14.7 & 720 & 46.0 & 54.3 & 0.48 & 4.2 & 1.1 & 166 & $<0.1$ & 3.1 & 0.3 & 0.8 & 37 & 0.4 & 4.6 & 220 & 0.7 & 23.30 \\
\hline V-03-10A & 2003 & 48.51792 & 92.69938 & yan Lake & 1,130 & 0.55 & 2.9 & 8.8 & 650 & 42.0 & 39.9 & 0.54 & 3.4 & 0.9 & 135 & $<0.1$ & 2.7 & 0.2 & 0.7 & 26 & 0.4 & 3.8 & 57 & 0.5 & 5.90 \\
\hline V-03-11A & 2003 & 48.51568 & -92.70500 & yan Lake & 1,490 & 0.51 & 3.8 & 13.3 & 580 & 38.0 & 56.0 & 0.42 & 4.3 & 0.9 & 152 & $<0.1$ & 3.0 & 0.2 & 0.8 & 35 & 0.3 & 4.4 & 61 & 0.4 & 8.90 \\
\hline V-03-11A dup & 2003 & 48.51568 & -92.70500 & yan Lake & 1,610 & 0.59 & 4.1 & 15.6 & 640 & 42.0 & 60.6 & 0.46 & 4.6 & 1.2 & 155 & $<0.1$ & 3.2 & 0.3 & 0.8 & 37 & 0.4 & 4.7 & 66 & 0.4 & 19.90 \\
\hline V-03- & 2003 & 48.51580 & -92.70547 & yan Lake & 232 & 0.62 & 5.2 & 21.3 & 200 & 35.7 & 58.5 & 0.35 & 5.7 & 1.1 & 211 & $<0.1$ & 3.1 & 0.3 & 1.0 & 54 & 0.4 & 5.6 & 46 & 0.3 & .58 \\
\hline V-03-13A & 2003 & 48.51373 & -92.70505 & yan Lake & 821 & 0.69 & 4.8 & 19.1 & 600 & 44.6 & 57.4 & 0.50 & 5.1 & 1.5 & 146 & $<0.1$ & 3.1 & 0.3 & 0.9 & 49 & 0.5 & 4.9 & 76 & 0.5 & 21.00 \\
\hline V-03-14A & 2003 & 48.51351 & -92.70242 & an Lake & 2,270 & 0.53 & 4.2 & 13.0 & 580 & 47.4 & 66.9 & 0.47 & 4.6 & 1.1 & 188 & $<0.1$ & 3.1 & 0.4 & 0.9 & 34 & 0.4 & 5.3 & 70 & 0.4 & 19.40 \\
\hline V-03-15A & 2003 & 48.51373 & 2.78778 & own Lake & 2,260 & 0.47 & 3.9 & 14.8 & 580 & 39.2 & 93.9 & 0.63 & 4.2 & 1.0 & 231 & $<0.1$ & 2.6 & 0.2 & 0.8 & 32 & 0.3 & 5.1 & 40 & 0.3 & 2.10 \\
\hline V-03 & 2003 & 48.51392 & -92.78711 & ke & 3,150 & 1.05 & 5.1 & 23.3 & 960 & 57.2 & 69.1 & 0.66 & 5.0 & 1.7 & 148 & $<0.1$ & 4.0 & 0.2 & 1.2 & 46 & 0.6 & 6.0 & 90 & 0.7 & 20.60 \\
\hline V-03-17A & 2003 & 48.51284 & -92.78577 & Brown Lake & 3,120 & 1.49 & 7.4 & 37.4 & 1,630 & 48.1 & 69.0 & 0.65 & 7.0 & 1.7 & 135 & $<0.1$ & 5.6 & 0.5 & 2.9 & 58 & 0.7 & 10.0 & 105 & 1.0 & 16.60 \\
\hline V-03-18A & 2003 & 48.51218 & -92.78512 & own Lake & 1,290 & 0.80 & 4.6 & 31.4 & 1,320 & 43.8 & 44.6 & 0.55 & 4.9 & 1.4 & 152 & $<0.1$ & 3.5 & 0.2 & 1.0 & 46 & 0.6 & 5.1 & 207 & 0.5 & 23.60 \\
\hline V-06-01A & 2006 & 51472 & 2.70396 & an Lake & 916 & 1.57 & 4.6 & 18.9 & 700 & 44.8 & 42.5 & 0.56 & 5.1 & 0.9 & 145 & $<0.1$ & 4.0 & 0.3 & 1.2 & 54 & 6 & 5.8 & 65 & 0.6 & 9.00 \\
\hline & & & 47 & & 177 & 0.63 & 3.0 & 12.8 & 430 & 44.7 & 36.1 & 0.53 & 3.9 & 0.5 & 130 & $<0.1$ & 2.7 & 0.2 & 0.9 & 33 & 3 & 4.6 & 28 & 6 & 2.80 \\
\hline V-06-03A & 2006 & 48.51574 & 92.70515 & yan Lake & 978 & 0.98 & 5.5 & 18.2 & 520 & 47.9 & 63.8 & 0.46 & 6.0 & 1.2 & 190 & $<0.1$ & 4.6 & 0.4 & 1.5 & 63 & 0.7 & 6.4 & 75 & 0.5 & 11.30 \\
\hline V-06-04A & 2006 & 48.51947 & -92.70412 & an Lake & 2,320 & 0.59 & 3.4 & 10.7 & 730 & 47.1 & 59.9 & 0.46 & 4.0 & 1.2 & 147 & $<0.1$ & 2.6 & 0.4 & 0.8 & 32 & 0.4 & 4.7 & 94 & 0.6 & 20.90 \\
\hline V-06-05A & 2006 & 52139 & -92.70499 & an Lake & 6,570 & 1.54 & 5.2 & 25.8 & 890 & 33.5 & 69.4 & 0.46 & 8.1 & 1.2 & 141 & $<0.1$ & 8.6 & 0.6 & 2.3 & 63 & 0.5 & 9.9 & 62 & 1.0 & 3.70 \\
\hline & 20 & & & & 319 & 0.4 & 3. & 9.0 & 410 & 25.0 & 58.8 & 32 & 4.3 & 0.9 & 195 & & 2.9 & 0.4 & 1.1 & & 14.5 & 6.7 & 27 & 3 & .70 \\
\hline & & & & & 1,220 & 0.69 & 3.9 & 15.4 & 650 & 29.9 & 74.5 & 0.42 & 5.5 & 1.1 & 174 & $<0.1$ & 3.5 & 0.4 & 1.1 & 45 & 0.6 & 5.1 & 56 & 0.5 & 14.80 \\
\hline V-06-08A & 2006 & 51816 & -92.70107 & an Lake & 1,070 & 1.18 & 4. & 14.8 & 620 & 35.5 & 62.4 & 0.34 & 5.6 & 1.2 & 215 & $<0.1$ & 3.5 & 0.4 & 1.0 & 50 & 0.5 & 6.1 & 102 & 0.3 & 13.30 \\
\hline V-06-09A & 2006 & 48.51760 & -92.70024 & an Lake & 206 & 0.49 & 2.5 & 8.5 & 570 & 31.1 & 32.3 & 0.30 & 3.4 & 0.8 & 126 & $<0.1$ & 2.2 & 0.3 & 0.7 & 26 & 0.4 & 3.9 & 31 & 0.6 & 25.70 \\
\hline & 2006 & 1627 & -92.70162 & & 2,310 & 0.74 & 3.4 & 16.2 & 740 & 49.4 & 60.0 & 0.55 & 3.9 & 1.3 & 151 & $<0.1$ & 2.7 & 0.4 & 0. & $3 s$ & 0.5 & 4.4 & 107 & 0.8 & 4.30 \\
\hline & & & & & & 0. & & 8.9 & 670 & & 64.8 & & 4. & & & & & & & & & 5.6 & 62 & 0.4 & 17.70 \\
\hline & 2006 & & -92.69928 & & 366 & 0.6 & 2. & 7.6 & 660 & 32.3 & 36.9 & 0.41 & 3. & 0. & 121 & $<0.1$ & 2.4 & 0.2 & 0. & 26 & 0.3 & 4.0 & 31 & 0.6 & 26.20 \\
\hline V-06-13A & 2006 & 48.51214 & -92.78509 & Brown Lake & 1,070 & 0.85 & 5.2 & 30.3 & 1,300 & 32.6 & 49.3 & 0.32 & 6.8 & 1.3 & 206 & $<0.1$ & 4.0 & 0.3 & 1.4 & 63 & 0.6 & 6.1 & 192 & 0.4 & 13.40 \\
\hline V-06-14A & 2006 & 1289 & -92.78571 & Brown Lake & 2,950 & 1.82 & 4.9 & 39.6 & 2,030 & 35.2 & 56.8 & 0.51 & 6.4 & 1.3 & 115 & $<0.1$ & 5.9 & 0.8 & 4.6 & 50 & 0.6 & 17.8 & 79 & 1.4 & 18.00 \\
\hline & & & -92.78703 & & 1,100 & 0.39 & 3. & 10.9 & 430 & 28.2 & 83.2 & 0.18 & 4.7 & 0. & 238 & $<0.1$ & 3. & 0.4 & 1. & & 0.3 & 5.4 & 29 & 0.2 & .67 \\
\hline $\mathrm{V}$ & 2006 & & -92.78757 & & 1,060 & 0.51 & 4.8 & 13.6 & 430 & 27.7 & 84.2 & 0.2 & 4.6 & 0.8 & 241 & $<0.1$ & 3.7 & 0.4 & 0.9 & 33 & 0.3 & 5.5 & 29 & 0.2 & 6.91 \\
\hline V-06-16A & 2006 & 48.51383 & -92.78757 & Brown Lake & 2,500 & 0.86 & 4.3 & 22.2 & 820 & 43.8 & 70.8 & 0.35 & 4.7 & 1.3 & 171 & $<0.1$ & 4.1 & 0.4 & 1.2 & 40 & 0.4 & 6.6 & 88 & 0.6 & 17.40 \\
\hline
\end{tabular}


Click back to Contents

Appendix 2-B Horizon Chemistry, Ryan and Brown Lakes, Voyageurs National Park, MN

\begin{tabular}{|c|c|c|c|c|c|c|c|c|c|c|c|c|c|c|c|c|c|c|c|c|c|c|c|c|c|c|c|c|}
\hline Field no. & $\begin{array}{c}\text { Year } \\
\text { collected }\end{array}$ & Latitude & Longitude & Watershed & $\begin{array}{c}\text { Al } \\
\text { (wt. \%) }\end{array}$ & $\begin{array}{c}\mathrm{Ca} \\
\text { (wt. \%) }\end{array}$ & $\begin{array}{c}\mathrm{Fe} \\
\text { (wt. \%) }\end{array}$ & $\begin{array}{c}\mathrm{K} \\
\text { (wt. \%) }\end{array}$ & \begin{tabular}{|c|}
$\mathrm{Mg}$ \\
(wt. \%)
\end{tabular} & $\begin{array}{c}\mathrm{Na} \\
\text { (wt. \%) }\end{array}$ & $\begin{array}{c}\mathrm{S} \\
\text { (wt. \%) }\end{array}$ & \begin{tabular}{|c|}
$\mathrm{Ti}$ \\
(wt. \%)
\end{tabular} & \begin{tabular}{|c|}
$\begin{array}{c}\mathrm{Ag} \\
(\mathrm{ppm})\end{array}$ \\
\end{tabular} & \begin{tabular}{|c} 
As \\
$(\mathrm{ppm})$
\end{tabular} & \begin{tabular}{|c|}
$\mathrm{Ba}$ \\
$(\mathrm{ppm})$
\end{tabular} & \begin{tabular}{|c|}
$\mathrm{Be}$ \\
$(\mathrm{ppm})$
\end{tabular} & \begin{tabular}{|c|}
$\mathrm{Bi}$ \\
$(\mathrm{ppm})$
\end{tabular} & \begin{tabular}{|c|}
$\mathrm{Cd}$ \\
$(\mathrm{ppm})$ \\
\end{tabular} & $\begin{array}{c}\mathrm{Ce} \\
(\mathrm{ppm})\end{array}$ & $\begin{array}{c}\text { Co } \\
\text { (ppm) }\end{array}$ & \begin{tabular}{|c|}
$\mathrm{Cr}$ \\
$(\mathrm{ppm})$
\end{tabular} & \begin{tabular}{|c|}
$\mathrm{CS}^{*}$ \\
$(\mathrm{ppm})$ \\
\end{tabular} & \begin{tabular}{|c|}
$\mathrm{Cu}$ \\
$(\mathrm{ppm})$
\end{tabular} & \begin{tabular}{|c|}
$G a$ \\
$(p p m)$
\end{tabular} & \begin{tabular}{|c|}
$\mathrm{Hg}$ \\
$(\mathrm{ppm})$
\end{tabular} & \begin{tabular}{c|} 
In \\
$(\mathrm{ppm})$
\end{tabular} & $\begin{array}{c}\mathrm{La} \\
(\mathrm{ppm})\end{array}$ & $\begin{array}{c}\mathrm{Li} \\
(\mathrm{ppm})\end{array}$ \\
\hline V-03-01B & 2003 & 48.51360 & -92.70532 & Ryan Lake & 5.75 & 1.05 & 2.32 & 1.31 & \begin{tabular}{|l|l|}
0.52 \\
\end{tabular} & 1.43 & 0.03 & 0.24 & $<1$ & 4 & 558 & $\begin{array}{ll}1.1 \\
\end{array}$ & \begin{tabular}{|l|}
0.22 \\
\end{tabular} & \begin{tabular}{|l|}
0.2 \\
\end{tabular} & 32.5 & 12.5 & 45 & 2.54 & 15.5 & 13.0 & \begin{tabular}{|l|}
0.07 \\
\end{tabular} & 0.03 & 16.4 & 25 \\
\hline V-03-02B & 2003 & 48.52164 & -92.70490 & Ryan Lake & 5.49 & 0.65 & 2.82 & 1.27 & 0.52 & 0.90 & 0.05 & 0.24 & $<1$ & 5 & 552 & 1.4 & 0.23 & 0.2 & 72.0 & 43.7 & 56 & 4.30 & 28.0 & 12.1 & 0.08 & 0.04 & 30.4 & 32 \\
\hline V-03-03B & 2003 & 48.52124 & 2.70567 & Ryan Lake & 5.54 & 1.10 & 1.03 & 1.66 & 0.31 & 1.89 & 0.02 & 0.17 & $<1$ & 1 & 462 & 1.0 & 0.14 & $<0.1$ & 27.8 & 4.3 & 27 & 1.76 & 10.6 & 11.5 & 0.03 & 0.02 & 16.0 & 8 \\
\hline V-03-04B & 2003 & 48.51987 & -92.70518 & Ryan Lake & 6.19 & 1.02 & 2.19 & 1.53 & 0.48 & 1.61 & 0.02 & 0.22 & $<1$ & 3 & 508 & 1.4 & 0.21 & $<0.1$ & 32.4 & 12.0 & 52 & 2.89 & 27.7 & 14.3 & 0.05 & 0.03 & 16.7 & 20 \\
\hline V-03-05B & 2003 & 48.51889 & -92.70403 & Ryan Lake & 5.53 & 1.15 & 1.55 & 1.63 & 0.40 & 1.70 & 0.02 & 0.24 & $<1$ & 3 & 544 & 1.0 & 0.17 & 0.1 & 21.9 & 6.1 & 37 & 1.72 & 14.4 & 13.1 & 0.03 & 0.02 & 11.3 & 14 \\
\hline V-03-06B & 2003 & 48.51519 & -92.70383 & Ryan Lake & 6.12 & 0.74 & 3.34 & 1.01 & 0.53 & 1.09 & 0.04 & 0.30 & $<1$ & 7 & 556 & 1.3 & 0.31 & 0.2 & 33.6 & 12.1 & 80 & 4.20 & 26.6 & 15.6 & 0.10 & 0.04 & 17.9 & 35 \\
\hline V-03-07B & 2003 & 48.51681 & -92.70174 & Ryan Lake & 6.56 & 1.06 & 3.07 & 0.93 & 0.63 & 1.50 & 0.02 & 0.30 & $<1$ & 5 & 418 & 1.2 & 0.24 & 0.1 & 21.1 & 10.3 & 87 & 3.48 & 20.5 & 15.1 & 0.06 & 0.04 & 11.4 & 26 \\
\hline V-03-08B & 2003 & 48.51711 & -92.70057 & Ryan Lake & 5.62 & 1.14 & 2.33 & 1.29 & 0.46 & 1.77 & 0.02 & 0.22 & $<1$ & 3 & 433 & 1.0 & 0.16 & $<0.1$ & 13.7 & 6.5 & 55 & 1.04 & 19.5 & 13.0 & 0.05 & 0.02 & 7.6 & 11 \\
\hline V-03-09B & 2003 & 48.51831 & -92.70119 & Ryan Lake & 6.23 & 1.16 & 2.70 & 1.31 & 0.58 & 1.51 & 0.02 & 0.29 & $<1$ & 4 & 573 & 1.2 & 0.20 & 0.2 & 23.9 & 9.6 & 57 & 4.65 & 12.0 & 14.4 & 0.05 & 0.03 & 12.7 & 24 \\
\hline V-03-09B dup & 2003 & 48.51831 & -92.70119 & Ryan Lake & 6.17 & 1.15 & 2.62 & 1.35 & 0.60 & 1.47 & 0.02 & 0.28 & $<1$ & 4 & 573 & 1.2 & 0.21 & 0.2 & 20.4 & 9.4 & 56 & 2.54 & 12.5 & 14.8 & 0.05 & 0.04 & 11.1 & 24 \\
\hline V-03-10B & 2003 & 48.51792 & 2.69938 & Ryan Lake & 6.03 & 1.26 & 1.06 & 1.72 & 0.34 & 2.18 & 0.01 & 0.18 & $<1$ & 2 & 536 & 1.1 & 0.10 & $<0.1$ & 15.3 & 4.1 & 31 & 0.89 & 6.0 & 13.1 & 0.03 & 0.02 & 8.1 & 9 \\
\hline V-03-11B & 2003 & 48.51568 & -92.70500 & Ryan Lake & 5.61 & 1.13 & 1.67 & 1.64 & 0.38 & 1.77 & 0.02 & 0.21 & 2 & 4 & 515 & 1.0 & 0.16 & $<0.1$ & 17.4 & 6.5 & 35 & 2.33 & 10.2 & 12.1 & 0.04 & 0.03 & 9.2 & 14 \\
\hline V-03-12B & 2003 & 48.51580 & -92.70547 & Ryan Lake & 6.44 & 1.14 & 2.19 & 1.60 & 0.53 & 1.92 & 0.01 & 0.23 & $<1$ & 2 & 455 & 1.2 & 0.17 & $<0.1$ & 18.6 & 8.3 & 53 & 2.84 & 13.8 & 14.8 & 0.04 & 0.03 & 10.2 & 26 \\
\hline V-03-13B & 2003 & 48.51373 & -92.70505 & Ryan Lake & 6.06 & 1.19 & 2.67 & 1.30 & 0.58 & 1.60 & 0.03 & 0.27 & $<1$ & 5 & 474 & 1.0 & 0.23 & 0.2 & 21.0 & 9.4 & 53 & 2.99 & 13.2 & 13.6 & 0.06 & 0.03 & 10.3 & 26 \\
\hline V-03-14B & 2003 & 48.51351 & -92.70242 & Ryan Lake & 5.85 & 1.28 & 1.67 & 1.81 & 0.48 & 1.96 & 0.01 & 0.21 & $<1$ & 2 & 506 & 1.1 & 0.11 & $<0.1$ & 16.0 & 9.5 & 40 & 2.19 & 6.1 & 13.1 & 0.02 & 0.02 & 8.4 & 15 \\
\hline V-03-14B dup & 2003 & 48.51351 & -92.70242 & Ryan Lake & 5.95 & 1.32 & 1.65 & 1.85 & 0.49 & 1.99 & 0.01 & 0.21 & $<1$ & 2 & 541 & 1.1 & 0.14 & 0.1 & 15.4 & 8.9 & 39 & 1.77 & 6.9 & 12.9 & 0.02 & 0.03 & 8.8 & 15 \\
\hline V-03-15B & 2003 & 48.51373 & -92.78778 & Brown Lake & 6.15 & 1.14 & 1.37 & 1.91 & 0.41 & 2.20 & 0.01 & 0.16 & $<1$ & 2 & 518 & 1.3 & 0.16 & $<0.1$ & 17.6 & 7.4 & 36 & 2.19 & 5.4 & 13.0 & 0.03 & $<0.02$ & 9.1 & 15 \\
\hline V-03-16B & 2003 & 48.51392 & -92.78711 & Brown Lake & 5.91 & 0.79 & 2.59 & 1.64 & 0.54 & 1.38 & 0.02 & 0.27 & $<1$ & 4 & 572 & 1.3 & 0.28 & 0.1 & 35.2 & 16.3 & 52 & 3.17 & 19.7 & 15.7 & 0.05 & 0.03 & 18.5 & 30 \\
\hline V-03-17B & 2003 & 48.51284 & -92.78577 & Brown Lake & 6.46 & 0.76 & 3.00 & 1.29 & 0.58 & 1.17 & 0.04 & 0.31 & $<1$ & 4 & 607 & 1.8 & 0.34 & 0.2 & 65.9 & 65.7 & 69 & 4.84 & 40.3 & 15.2 & 0.07 & 0.04 & 28.2 & 37 \\
\hline V-03-18B & 2003 & 48.51218 & -92.78512 & Brown Lake & 6.56 & 1.01 & 3.38 & 0.84 & 0.69 & 1.61 & 0.03 & 0.33 & $<1$ & 4 & 477 & 1.4 & 0.26 & 0.2 & 21.7 & 14.6 & 97 & 3.75 & 26.2 & 16.6 & 0.04 & 0.04 & 11.6 & 36 \\
\hline V-06-01B & 2006 & 48.51472 & -92.70396 & Ryan Lake & 6.74 & 0.89 & 3.82 & 0.97 & 0.55 & 1.23 & 0.03 & 0.30 & $<1$ & 6 & 424 & 1.5 & 0.23 & 0.2 & 24.9 & 13.0 & 81 & $<5$ & 29.6 & 18.0 & 0.08 & 0.04 & 13.6 & 38 \\
\hline V-06-02B & 2006 & 48.51580 & -92.70547 & Ryan Lake & 6.48 & 1.13 & 2.32 & 1.60 & 0.54 & 1.89 & 0.01 & 0.22 & $<1$ & 6 & 425 & 1.3 & 0.09 & $<0.1$ & 18.3 & 8.8 & 62 & $<5$ & 13.8 & 15.9 & 0.03 & 0.03 & 10.1 & 25 \\
\hline V-06-03B & 2006 & 48.51574 & -92.70515 & Ryan Lake & 6.67 & 1.05 & 3.23 & 1.13 & 0.63 & 1.75 & 0.02 & 0.29 & $<1$ & 4 & 410 & 1.5 & 0.22 & 0.1 & 28.0 & 12.3 & 74 & $<5$ & 19.6 & 17.2 & 0.04 & 0.03 & 14.9 & 30 \\
\hline V-06-04B & 2006 & 48.51947 & -92.70412 & Ryan Lake & 5.89 & 1.12 & 2.19 & 1.67 & 0.52 & 1.61 & 0.01 & 0.23 & $<1$ & 4 & 483 & 1.4 & 0.08 & $<0.1$ & 18.4 & 8.4 & 50 & $<5$ & 9.1 & 14.1 & 0.03 & 0.03 & 10.1 & 21 \\
\hline V-06-05B & 2006 & 48.52139 & -92.70499 & Ryan Lake & 6.75 & 0.88 & 3.08 & 1.95 & 0.66 & 1.39 & 0.02 & 0.26 & $<1$ & 7 & 641 & 1.4 & 0.15 & 0.1 & 56.1 & 47.8 & 75 & 6 & 18.5 & 14.3 & 0.03 & 0.04 & 20.1 & 40 \\
\hline V-06-06B & 2006 & 48.52126 & -92.70521 & Ryan Lake & 6.07 & 1.20 & 0.87 & 2.17 & 0.26 & 2.33 & $<0.01$ & 0.14 & $<1$ & 1 & 485 & 1.4 & 0.02 & $<0.1$ & 17.7 & 2.6 & 26 & $<5$ & 3.8 & 12.4 & 0.01 & $<0.02$ & 8.8 & 5 \\
\hline V-06-07B & 2006 & 48.52016 & -92.70523 & Ryan Lake & 8.06 & 1.28 & 3.25 & 2.08 & 0.63 & 2.03 & 0.02 & 0.26 & $<1$ & 5 & 576 & 1.9 & 0.14 & 0.2 & 32.6 & 27.8 & 69 & 5 & 19.4 & 19.0 & 0.04 & 0.04 & 16.0 & 28 \\
\hline V-06-08B & 2006 & 48.51816 & -92.70107 & Ryan Lake & 7.07 & 1.33 & 2.85 & 1.68 & 0.63 & 1.89 & 0.02 & 0.27 & $<1$ & 3 & 507 & 1.3 & 0.10 & 0.1 & 21.3 & 9.0 & 57 & $<5$ & 9.3 & 15.7 & 0.03 & 0.03 & 10.1 & 24 \\
\hline V-06-09B & 2006 & 48.51760 & -92.70024 & Ryan Lake & 7.12 & 1.73 & 2.25 & 1.78 & 0.58 & 2.35 & 0.01 & 0.28 & $<1$ & 19 & 481 & 1.2 & 0.10 & 0.1 & 17.8 & 7.1 & 51 & $<5$ & 10.1 & 16.4 & 0.03 & 0.04 & 8.9 & 9 \\
\hline V-06-10B & 2006 & 48.51627 & -92.70162 & Ryan Lake & 7.61 & 1.34 & 3.47 & 1.04 & 0.78 & 1.94 & 0.01 & 0.34 & $<1$ & 5 & 429 & 1.8 & 0.18 & 0.1 & 24.1 & 13.5 & 90 & $<5$ & 15.2 & 18.1 & 0.03 & 0.04 & 12.2 & 28 \\
\hline V-06-11B & 2006 & 48.51359 & -92.70248 & Ryan Lake & 6.93 & 1.70 & 1.97 & 2.18 & 0.59 & 2.18 & 0.01 & 0.23 & $<1$ & 3 & 540 & 1.3 & 0.05 & $<0.1$ & 20.4 & 7.6 & 45 & $<5$ & 6.4 & 14.9 & 0.01 & 0.02 & 10.4 & 12 \\
\hline V-06-12B & 2006 & 48.71781 & -92.69928 & Ryan Lake & 7.57 & 1.47 & 1.90 & 2.12 & 0.54 & 2.34 & 0.02 & 0.26 & $<1$ & 4 & 580 & 1.3 & 0.05 & 0.1 & 28.2 & 8.5 & 45 & 5 & 9.9 & 18.3 & 0.02 & 0.03 & 14.0 & 17 \\
\hline V-06-13B & 2006 & 48.51214 & -92.78509 & Brown Lake & 8.24 & 1.32 & 4.38 & 1.05 & 1.08 & 2.03 & 0.02 & 0.38 & $<1$ & 5 & 477 & 1.5 & 0.19 & 0.2 & 29.1 & 19.0 & 129 & 6 & 26.0 & 20.6 & 0.02 & 0.04 & 14.4 & 47 \\
\hline V-06-14B & 2006 & 48.51289 & -92.78571 & Brown Lake & 7.90 & 0.97 & 3.62 & 1.54 & 0.78 & 1.37 & 0.04 & 0.32 & $<1$ & 5 & 584 & 1.9 & 0.23 & 0.2 & 79.1 & 69.0 & 85 & 7 & 53.6 & 16.9 & 0.07 & 0.06 & 34.8 & 43 \\
\hline V-06-15B & 2006 & 48.51385 & -92.78703 & Brown Lake & 6.48 & 1.12 & 2.49 & 1.84 & 0.64 & 1.74 & 0.02 & 0.23 & $<1$ & 5 & 537 & 1.4 & 0.32 & 0.2 & 27.8 & 11.2 & 64 & $<5$ & 13.3 & 14.4 & 0.04 & 0.03 & 14.0 & 27 \\
\hline V-06-15B dup & 2006 & 48.51385 & -92.78703 & Brown Lake & 6.66 & 1.14 & 2.55 & 1.91 & 0.66 & 1.80 & 0.02 & 0.23 & $<1$ & 5 & 556 & 1.8 & 0.19 & 0.2 & 27.9 & 12.3 & 54 & $<5$ & 12.8 & 16.5 & 0.04 & 0.04 & 14.0 & 28 \\
\hline V-06-16B & 2006 & 48.51383 & -92.78757 & Brown Lake & 7.99 & 1.47 & 1.95 & 2.47 & 0.60 & 2.68 & 0.01 & 0.20 & $<1$ & 3 & 533 & 2.1 & 0.20 & $<0.1$ & 23.7 & 8.8 & 46 & 5 & 5.4 & 17.5 & 0.01 & 0.03 & 12.1 & 20 \\
\hline
\end{tabular}

${ }^{*}$ Because of analytical laboratory issues, Cs detection limits in 2003 were $0.05 \mathrm{ppm}$ and 5 ppm in 2006. 
Appendix 2-B Horizon Chemistry, Ryan and Brown Lakes, Voyageurs National Park, MN

\begin{tabular}{|c|c|c|c|c|c|c|c|c|c|c|c|c|c|c|c|c|c|c|c|c|c|c|c|c|c|}
\hline Field no. & $\begin{array}{c}\text { Year } \\
\text { collected }\end{array}$ & atitude & ongitude & Watershed & $\begin{array}{c}\mathrm{Mn} \\
(\mathrm{ppm})\end{array}$ & $\begin{array}{c}\text { Mo } \\
(\mathrm{ppm})\end{array}$ & $\begin{array}{c}\mathrm{Nb} \\
(\mathrm{ppm})\end{array}$ & $\begin{array}{c}\mathrm{Ni} \\
(\mathrm{ppm})\end{array}$ & \begin{tabular}{|c|}
$P$ \\
$(\mathrm{ppm})$
\end{tabular} & $\begin{array}{c}\mathrm{Pb} \\
(\mathrm{ppm})\end{array}$ & $\begin{array}{c}\mathrm{Rb} \\
(\mathrm{ppm})\end{array}$ & \begin{tabular}{|c|}
$\mathrm{Sb}$ \\
$(\mathrm{ppm})$
\end{tabular} & \begin{tabular}{|c|}
$\mathrm{Sc}$ \\
$(\mathrm{ppm})$
\end{tabular} & $\begin{array}{c}\text { Sn } \\
(\mathrm{ppm})\end{array}$ & $\begin{array}{c}\mathrm{Sr} \\
(\mathrm{ppm})\end{array}$ & \begin{tabular}{|c|}
$\mathrm{Te}$ \\
$(\mathrm{ppm})$
\end{tabular} & \begin{tabular}{|c|} 
Th \\
$(\mathrm{ppm})$
\end{tabular} & $\begin{array}{c}\mathrm{Tl} \\
(\mathrm{ppm})\end{array}$ & $\begin{array}{c}\mathbf{U} \\
(\mathrm{ppm})\end{array}$ & $\begin{array}{c}V \\
(\mathrm{ppm})\end{array}$ & $\begin{array}{c}W \\
(\mathrm{ppm})\end{array}$ & $\begin{array}{c}\mathrm{Y} \\
(\mathrm{ppm})\end{array}$ & $\begin{array}{c}\mathrm{Zn} \\
(\mathrm{ppm})\end{array}$ & $\begin{array}{c}\mathrm{Se} \\
(\mathrm{ppm})\end{array}$ & $\begin{array}{l}\text { Total C } \\
\text { (wt. \%) }\end{array}$ \\
\hline$-03-01 B$ & 2003 & 48.51360 & .70532 & Ryan Lake & 1,700 & 0.71 & 6.4 & 29.3 & 800 & 28.0 & 72.2 & \begin{tabular}{|l|}
0.36 \\
\end{tabular} & \begin{tabular}{|l|}
6.8 \\
\end{tabular} & 1.2 & 204 & \begin{tabular}{|c|c|}
$<0.1$ \\
\end{tabular} & 5.0 & 0.4 & \begin{tabular}{|l|}
1.3 \\
\end{tabular} & $\begin{array}{r}62 \\
\end{array}$ & 0.6 & 7.4 & 113 & $\begin{array}{ll}0.4 \\
\end{array}$ & 5.73 \\
\hline & & & & & & & 6.8 & .7 & & & & 40 & 8.0 & .4 & 49 & 0.1 & 11.8 & 0.6 & 2.3 & 82 & 0.6 & 9.3 & 60 & 0.7 & 8.22 \\
\hline & & & & & & & 6.1 & 10.6 & 190 & 3.8 & 88.4 & & 5.4 & 0.8 & 44 & $<0.1$ & 5.0 & 0.5 & 2.1 & 34 & 0.4 & 8.3 & 25 & $<0.2$ & 3.97 \\
\hline & & 51987 & & an Lake & 36 & & 6.3 & 22.6 & 420 & 0.5 & 90.7 & 25 & 7.0 & 1.2 & 223 & $<0.1$ & 6.8 & 0.5 & 1.8 & 63 & 0.6 & 6.8 & 45 & 0.3 & 8.50 \\
\hline & & & & ke & 439 & & 6.4 & 13.8 & 280 & & 83.5 & 26 & 6.1 & 1.1 & 40 & $<0.1$ & 3.9 & 0.4 & 1.0 & 53 & 0.5 & 6.0 & 47 & $<0.2$ & 3.01 \\
\hline & & & & & 876 & & 8.1 & 37.8 & 650 & .8 & 65.4 & 52 & 7.5 & 1.6 & 174 & $<0.1$ & 6.0 & 0.4 & 1.7 & 94 & 1.0 & 6.6 & 135 & 0.6 & 7.45 \\
\hline & & & & & 45 & & 7.4 & 4 & 420 & & 0 & & 7.0 & 1.4 & 31 & $<0.1$ & 5.0 & 0.3 & 1.3 & 85 & 1.3 & 6.5 & 77 & .4 & .25 \\
\hline & & & & & & & 5.8 & & & & & & & & & & & 0.3 & 1.0 & & & & & .4 & .41 \\
\hline & & & & & 72 & & 7.5 & 8 & 530 & & .1 & & & 3 & 242 & 0.1 & 5.2 & 0.3 & 1.2 & 80 & 0.6 & 6.9 & 110 & 0.3 & 1.06 \\
\hline & & & & & & & 7.4 & & & & 3 & & & 1.4 & 36 & $<0.1$ & 5.2 & 0.4 & 1.1 & 78 & 0.7 & 5.9 & 108 & 0.3 & 4.05 \\
\hline & & & & & & & 5.2 & 14.0 & 150 & & & & & & 31 & $<0.1$ & 7.7 & 0.4 & 0.9 & 41 & 0.4 & 5.0 & 27 & $<0.2$ & 2.92 \\
\hline & & & & & 45 & & 5.4 & 2 & 290 & & 3 & & & 0.9 & 35 & $<0.1$ & 3.8 & 0.4 & 1.0 & 51 & 0.4 & 5.8 & 40 & 0.2 & 5.02 \\
\hline & & & & & & & & & & & & & & & & & & & & & & & & & .91 \\
\hline & & & & & & & 6 & & & & & & & & 36 & $<$ & 4. & 0.4 & & 75 & .5 & 6.9 & & 0.3 & .76 \\
\hline & & & & & & & & & & & & & & & & 0.1 & 3. & 0.4 & 1. & & .3 & 5.8 & 39 & 0.2 & 2.03 \\
\hline & & & & & & & 5 & & & & & & & & & .1 & & & & & & & 39 & 0.2 & 2.04 \\
\hline & & & & & & & 4.9 & & 290 & & 104.0 & & & & 73 & 0.1 & 6.3 & 0.4 & 0. & & 0.4 & 5.5 & 51 & $<0.2$ & .62 \\
\hline & & & & & & & 7.6 & .8 & & & & & & & & $<0.1$ & & 0.5 & 1. & & 0.7 & 6.8 & 28 & 0.5 & 5.20 \\
\hline & & & & & 2,560 & & 8.9 & 54 & & & 9 & & & & & $<$ & 7.7 & 0.8 & 3. & 84 & 0.9 & 11.2 & & 0 & 4.87 \\
\hline & & & & & & & 8.1 & .7 & 80 & & & & & & & $<0.1$ & 5.0 & 0.3 & 1.5 & 90 & 0.8 & 6.9 & 204 & 0.4 & 5.22 \\
\hline & & & & & 520 & & 7.5 & 38.1 & \begin{tabular}{|l|} 
\\
550
\end{tabular} & & 3.5 & & & 1.6 & 79 & $<0.1$ & 5.5 & 0.4 & 1.6 & 103 & 0.8 & 6.1 & 86 & 0.7 & 5.32 \\
\hline & & & & & 252 & & 4.9 & 29.2 & 150 & & 7 & & & & 234 & $<0.1$ & 8.4 & 0.4 & 1.2 & 70 & 0.3 & 5.8 & 39 & $<0.2$ & 2.37 \\
\hline & & & & yar & & 1.5 & 6.8 & 28. & 470 & 30 & 65 & & & 1.4 & 236 & $<0.1$ & 5.3 & 0.4 & 1.5 & 93 & 0.6 & 6.3 & 91 & 0.3 & 2.80 \\
\hline & 200 & & & & 468 & & 5.6 & 16.7 & 54 & & 79 & & & 1.1 & 17 & $<0.1$ & 3.9 & 0.4 & 1.1 & 65 & 0.4 & 5.6 & 63 & 0.3 & 2.73 \\
\hline & 2006 & 52139 & & yan Lake & 3,470 & 1.84 & 6.9 & 29.6 & 440 & & 99.3 & & & 1.4 & 00 & $<0.1$ & 12.5 & 0.7 & 2.3 & 98 & 0.6 & 7.8 & 64 & 0.6 & 2.28 \\
\hline & & & & & 229 & 0. & 3.3 & 5.7 & 110 & & 88.3 & & & 0.6 & 266 & $<0.1$ & 4.5 & 0.5 & 1.1 & 28 & 0.2 & 5.8 & 19 & $<0.2$ & 1.23 \\
\hline & & & & & & & 6.8 & & 50 & & 107.0 & & & & & $<0.1$ & & & & & & & & & 3.39 \\
\hline & & & & & & & 6. & & & & & & & & 62 & $<0.1$ & 5.0 & 0.4 & 1.3 & 79 & 0.5 & 6.6 & 78 & $<0.2$ & 2.9 \\
\hline & 2006 & 1760 & & yan Lake & 357 & 1.18 & 5.6 & 17.2 & 180 & & 75.6 & 0. & & 1.2 & 317 & $<0.1$ & 5.0 & 0.5 & 1.4 & 75 & 0.4 & 8.1 & 39 & $<0.2$ & 3.07 \\
\hline & & & & & $4 \mathrm{~s}$ & & 6.9 & 35.9 & 430 & 34.2 & 57.4 & & & 1. & 283 & $<0.1$ & 5.9 & 0.3 & 1.6 & 95 & 0.7 & 6.6 & 91 & 0.2 & 2.97 \\
\hline & & & & & & & 4.9 & & 200 & 23.4 & & & & & & $<0.1$ & 4.6 & 0.5 & & J & 0.3 & + & נכ & $<0.2$ & 1.97 \\
\hline & & & & & & & & & & & & & & & & $<0.1$ & & & 1.6 & & & 8.7 & 36 & $<0.2$ & 3.1 \\
\hline V & 2006 & 1214 & .78509 & own Lake & 551 & 1. & 8.2 & 45.7 & $\mid 1,070$ & .8 & 59.6 & 0. & 10.6 & 1.6 & 283 & $<0.1$ & 7.9 & 0.4 & 2.2 & 113 & 0.8 & 8.8 & 212 & 0.3 & 2.61 \\
\hline & 2006 & & .78571 & Brown Lake & 1,710 & 2.4 & 7.7 & 78.8 & $\mid 1,870$ & & 89.3 & 0.3 & 10.4 & 1. & 191 & $<0.1$ & 10.7 & 0.7 & 4.8 & 94 & 0.7 & 15.3 & 146 & 1.0 & 4.39 \\
\hline & & & & & 763 & 0.9 & 6.2 & 30.5 & 970 & & & & & & & $<0.1$ & 5.4 & 0.0 & 1.6 & 33 & & 7.8 & 113 & 0.3 & 4.7 \\
\hline & & & & & 793 & 0.80 & 7.0 & 26.6 & 1,030 & 22.6 & & & 1. & 1. & 235 & $<0.1$ & 0. & 0.5 & 1. & 00 & 6.5 & 9.0 & 109 & 0.3 & 3.0 \\
\hline V-06-16B & 2006 & .51383 & -92.78757 & Brown Lake & 511 & 0.47 & 16.9 & 17.0 & 360 & 22.0 & 120.0 & 0.17 & 7.1 & 1.1 & 322 & $<0.1$ & 7.5 & 0.6 & 5.0 & 48 & 0.6 & 19.6 & 47 & $<0.2$ & 1.2 \\
\hline
\end{tabular}


Click back to Contents

Appendix 3-O Horizon Chemistry, Ryan and Brown Lakes, Voyageurs National Park, MN

\begin{tabular}{|c|c|c|c|c|c|c|c|c|c|c|c|c|c|c|c|c|c|c|c|c|c|c|c|c|c|c|c|c|}
\hline Field no. & $\begin{array}{c}\text { Year } \\
\text { collected }\end{array}$ & Latitude & Longitude & Watershed & $\begin{array}{l}\text { Ash } \\
(\%)\end{array}$ & $\begin{array}{c}\text { Al } \\
\text { (wt. \%) }\end{array}$ & \begin{tabular}{|c|}
$\mathrm{Ca}$ \\
(wt. \%)
\end{tabular} & $\begin{array}{c}\mathrm{Fe} \\
\text { (wt. \%) }\end{array}$ & $\begin{array}{c}\mathrm{K} \\
\text { (wt. \%) }\end{array}$ & \begin{tabular}{|c|} 
Mg \\
(wt. \%)
\end{tabular} & \begin{tabular}{|c|}
$\mathrm{Na}$ \\
(wt. \%)
\end{tabular} & $\begin{array}{c}\mathrm{S} \\
\text { (wt. \%) }\end{array}$ & \begin{tabular}{|c|}
$\mathrm{Ti}$ \\
(wt. \%)
\end{tabular} & \begin{tabular}{|c|}
$\mathrm{Ag}$ \\
$(\mathrm{ppm})$
\end{tabular} & \begin{tabular}{|c|} 
As \\
$(\mathrm{ppm})$
\end{tabular} & \begin{tabular}{|c|}
$\mathrm{Ba}$ \\
$(\mathrm{ppm})$ \\
\end{tabular} & \begin{tabular}{|c|} 
Be \\
$(\mathrm{ppm})$
\end{tabular} & \begin{tabular}{|c|}
$\mathrm{Bi}$ \\
$(\mathrm{ppm})$
\end{tabular} & \begin{tabular}{|c|}
$\mathrm{Cd}$ \\
$(\mathrm{ppm})$ \\
\end{tabular} & \begin{tabular}{|c|}
$\mathrm{Ce}$ \\
$(\mathrm{ppm})$
\end{tabular} & \begin{tabular}{|c|} 
Co \\
$(\mathrm{ppm})$
\end{tabular} & $\begin{array}{c}\mathrm{Cr} \\
(\mathrm{ppm})\end{array}$ & \begin{tabular}{|c|}
$\mathrm{Cs}^{*}$ \\
$(\mathrm{ppm})$ \\
\end{tabular} & $\begin{array}{c}\mathrm{Cu} \\
(\mathrm{ppm})\end{array}$ & \begin{tabular}{|c|}
$\mathrm{Ga}$ \\
$(\mathrm{ppm})$ \\
\end{tabular} & \begin{tabular}{|c|}
$\begin{array}{c}\mathrm{Hg} \\
(\mathrm{ppm})\end{array}$ \\
\end{tabular} & \begin{tabular}{|c|} 
In \\
$(\mathrm{ppm})$
\end{tabular} & $\begin{array}{c}\text { La } \\
(\mathrm{ppm})\end{array}$ \\
\hline V-03-01O & 2003 & 48.51360 & -92.70532 & Ryan Lake & 25.15 & 5.69 & \begin{tabular}{|r|}
4.50 \\
\end{tabular} & 2.88 & 1.61 & 0.87 & \begin{tabular}{|l|}
1.16 \\
\end{tabular} & 0.28 & \begin{tabular}{|l|}
0.26 \\
\end{tabular} & \begin{tabular}{|r|}
$<1$ \\
$<1$
\end{tabular} & \begin{tabular}{|r|} 
\\
\end{tabular} & \begin{tabular}{|c|}
1,460 \\
\end{tabular} & \begin{tabular}{|r|}
2.0 \\
\end{tabular} & \begin{tabular}{|r|}
0.69 \\
0.69
\end{tabular} & \begin{tabular}{|r|}
3.2 \\
\end{tabular} & \begin{tabular}{|l|}
109.0 \\
\end{tabular} & \begin{tabular}{|l|}
25.5 \\
\end{tabular} & 43 & \begin{tabular}{|r|}
6.66 \\
\end{tabular} & \begin{tabular}{|r|}
41.3 \\
\end{tabular} & \begin{tabular}{|c|}
16.9 \\
\end{tabular} & \begin{tabular}{|r|}
0.15 \\
0.15
\end{tabular} & \begin{tabular}{|r|}
0.1 \\
\end{tabular} & 47.8 \\
\hline V-03-01O dup & 2003 & 48.51360 & -92.70532 & Ryan Lake & 20.60 & 3.56 & 11.10 & 1.60 & 1.47 & 1.46 & 0.40 & 0.78 & 0.12 & $<1$ & 4 & 2,380 & 5.4 & 0.30 & 5.9 & \begin{tabular}{|l|}
408.0 \\
\end{tabular} & 58.6 & 23 & 5.77 & 114.0 & 8.7 & 0.08 & 0.1 & 191.0 \\
\hline V-03-02O & 2003 & 48.52164 & -92.70490 & Ryan Lake & 11.05 & 4.60 & 5.33 & 1.46 & 1.74 & 0.74 & 1.25 & 0.28 & 0.17 & $<1$ & 4 & $\mid 1,210$ & 1.7 & 0.30 & 1.7 & $\mid 109.0$ & 30.8 & 33 & 3.92 & 43.0 & 11.1 & 0.09 & 0.0 & 59.8 \\
\hline V-03-03O & 2003 & 48.52124 & -92.70567 & Ryan Lake & 17.72 & 5.21 & 6.58 & 3.51 & 1.80 & 0.99 & 0.69 & 0.63 & 0.21 & $<1$ & 10 & $\mid 1,370$ & 2.5 & 0.60 & 3.4 & 208.0 & 132.0 & 57 & 6.95 & 77.3 & 13.4 & 0.12 & 0.1 & 93.7 \\
\hline V-03-04O & 2003 & 48.51987 & -92.70518 & Ryan Lake & 10.00 & 3.85 & 12.20 & 2.21 & 2.16 & 1.37 & 0.62 & 0.73 & 0.17 & $<1$ & 7 & $\mid 1,170$ & 1.0 & 0.62 & 4.8 & \begin{tabular}{|l|} 
\\
\end{tabular} & 13.4 & 32 & 5.66 & 61.1 & 9.4 & 0.12 & 0.1 & 15.4 \\
\hline V-03-05O & 2003 & 48.51889 & -92.70403 & Ryan Lake & 9.50 & 5.34 & 7.39 & 3.14 & 1.75 & 0.99 & 0.87 & 0.51 & 0.26 & $<1$ & 10 & $|1,660|$ & 1.5 & 0.69 & 7.2 & 42.2 & 20.7 & 53 & 9.68 & 54.4 & 15.4 & 0.16 & 0.1 & 22.2 \\
\hline V-03-06O & 2003 & 48.51324 & -92.70163 & Ryan Lake & 16.30 & 5.56 & 6.84 & 3.00 & 1.60 & 1.10 & 1.18 & 0.37 & 0.27 & $<1$ & 7 & 839 & 1.2 & 0.46 & 2.8 & 30.8 & 18.4 & 66 & 6.61 & 39.4 & 15.1 & 0.14 & 0.1 & 16.7 \\
\hline V-03-07O & 2003 & 48.51681 & -92.70174 & Ryan Lake & 18.70 & 2.61 & 13.90 & 1.52 & 1.92 & 2.22 & 0.55 & 1.08 & 0.11 & $<1$ & 3 & $\mid 1,640$ & 1.0 & 0.31 & 7.4 & 27.6 & 49.4 & 34 & 9.09 & $\mid 102.0$ & 6.6 & 0.09 & 0.0 & 15.1 \\
\hline V-03-08O & 2003 & 48.51711 & -92.70057 & Ryan Lake & 6.30 & 4.48 & 9.98 & 2.38 & 1.87 & 1.10 & 0.98 & 0.56 & 0.20 & $<1$ & 6 & 2,620 & 1.1 & 0.56 & 7.3 & 30.7 & 17.6 & 32 & 9.03 & 53.5 & 12.3 & 0.15 & 0.1 & 16.0 \\
\hline V-03-09O & 2003 & 48.51831 & -92.70119 & Ryan Lake & 14.80 & 3.20 & 11.10 & 1.39 & 1.80 & 1.77 & 0.77 & 0.76 & 0.14 & $<1$ & 5 & $\mid 1,720$ & 1.0 & 0.41 & 5.8 & 28.0 & 41.2 & 22 & 5.05 & 80.7 & 8.9 & 0.10 & 0.0 & 15.5 \\
\hline V-03-10O & 2003 & 48.51792 & -92.69938 & Ryan Lake & 11.60 & 4.75 & 6.77 & 1.75 & 2.14 & 1.05 & 1.39 & 0.35 & 0.18 & $<1$ & 4 & $\mid 1,730$ & 1.1 & 0.31 & 5.1 & 24.4 & 18.5 & 53 & 3.75 & 44.4 & 12.3 & 0.10 & 0.0 & 12.8 \\
\hline V-03-11O & 2003 & 48.51568 & -92.70500 & Ryan Lake & 17.75 & 5.48 & 4.80 & 2.88 & 1.70 & 0.86 & 1.22 & 0.65 & 0.23 & $<1$ & 8 & $\mid 1,200$ & 1.6 & 0.61 & 2.2 & 59.4 & 23.0 & 39 & 8.18 & 49.4 & 14.5 & 0.22 & 0.1 & 33.5 \\
\hline V-03-12O & 2003 & 48.51580 & -92.70547 & Ryan Lake & 13.65 & 5.66 & 3.54 & 2.97 & 1.81 & 0.78 & 1.31 & 0.31 & 0.26 & $<1$ & 8 & 813 & 1.2 & 0.59 & 1.9 & 32.9 & 14.5 & 39 & 7.70 & 30.9 & 15.8 & 0.19 & 0.1 & 16.6 \\
\hline V-03-13O & 2003 & 48.51373 & -92.70205 & Ryan Lake & 19.70 & 4.12 & 8.09 & 1.60 & 1.74 & 1.07 & 1.14 & 0.40 & 0.18 & $<1$ & 5 & $\mid 1,580$ & 1.2 & 0.42 & 4.5 & 39.6 & 35.9 & 23 & 4.92 & 49.6 & 11.2 & 0.09 & 0.1 & 22.5 \\
\hline V-03-14O & 2003 & 48.51351 & -92.70242 & Ryan Lake & 16.55 & 4.69 & 6.98 & 1.76 & 2.12 & 0.98 & 1.29 & 0.41 & 0.15 & $<1$ & 4 & $\mid 1,400$ & 1.1 & 0.42 & 2.8 & 32.7 & 12.3 & 26 & 4.32 & 35.7 & 11.6 & 0.13 & 0.1 & 18.3 \\
\hline V-03-15O & 2003 & 48.51373 & -92.78778 & Brown Lake & 21.10 & 4.92 & 7.76 & 2.67 & 2.01 & 1.19 & 0.98 & 0.53 & 0.21 & $<1$ & 6 & $\mid 1,220$ & 1.2 & 0.51 & 3.1 & 34.4 & 15.5 & 49 & 5.25 & 48.1 & 13.7 & 0.12 & 0.1 & 17.6 \\
\hline V-03-16O & 2003 & 48.51392 & -92.78711 & Brown Lake & 16.05 & 4.16 & 10.70 & 2.10 & 1.84 & 1.60 & 0.65 & 0.74 & 0.18 & $<1$ & 3 & 2,840 & 1.6 & 0.41 & 4.8 & $\mid 117.0$ & 43.0 & 34 & 5.06 & 95.6 & 10.8 & 0.09 & 0.0 & 54.6 \\
\hline V-03-160 dup & 2003 & 48.51392 & -92.78711 & Brown Lake & 17.95 & 2.34 & 15.60 & 1.49 & 1.58 & 2.03 & 0.44 & 0.88 & 0.11 & 1 & 4 & 2,620 & 0.8 & 0.27 & 9.4 & \begin{tabular}{|l|}
23.5 \\
\end{tabular} & 18.2 & 25 & 5.99 & 120.0 & 6.5 & 0.11 & 0.0 & 13.2 \\
\hline V-03-170 & 2003 & 48.51284 & -92.78577 & Brown Lake & 13.60 & 5.34 & 7.17 & 2.64 & 2.03 & 1.12 & 1.14 & 0.47 & 0.23 & $<1$ & 5 & $\mid 1,140$ & 1.3 & 0.42 & 2.4 & 32.6 & 16.1 & 36 & 5.36 & 43.6 & 15.1 & 0.11 & 0.1 & 16.2 \\
\hline V-03-18O & 2003 & 48.51218 & -92.78512 & Brown Lake & 11.40 & 5.71 & 5.05 & 2.81 & 1.68 & 0.88 & 1.20 & 0.34 & 0.25 & $<1$ & 8 & $\mid 1,440$ & 1.7 & 0.56 & 3.3 & 93.9 & 21.9 & 38 & 5.33 & 42.1 & 15.4 & 0.16 & 0.1 & 41.9 \\
\hline V-06-1 O & 2006 & 48.51472 & -92.70396 & Ryan Lake & 19.80 & 5.57 & 4.54 & 2.78 & 1.64 & 0.81 & 1.17 & 0.54 & 0.28 & $<1$ & 8 & $\mid 1,080$ & 1.3 & 0.68 & 2.7 & 44.8 & 18.0 & 48 & 12.00 & 44.1 & 18.1 & 0.17 & 0.1 & 22.8 \\
\hline V-06-2 O & 2006 & 48.51580 & -92.70547 & Ryan Lake & 12.50 & 5.87 & 6.32 & 2.62 & 1.74 & 1.01 & 1.18 & 0.89 & 0.19 & $<1$ & 7 & $\mid 1,230$ & 1.4 & 0.49 & 2.6 & 58.7 & 21.7 & 35 & 6.00 & 65.2 & 12.3 & 0.19 & 0.0 & 29.7 \\
\hline V-06-3 O & 2006 & 48.51574 & -92.70515 & Ryan Lake & 18.00 & 5.51 & 5.70 & 2.31 & 1.40 & 0.88 & 1.34 & 0.40 & 0.24 & $<1$ & 5 & $\mid 1,290$ & 1.1 & 0.46 & 2.2 & 33.3 & 18.3 & 40 & $<5$ & 44.2 & 15.3 & 0.10 & 0.0 & 17.1 \\
\hline V-06-4 O & 2006 & 48.51947 & -92.70412 & Ryan Lake & 11.70 & 4.62 & 10.80 & 2.11 & 2.00 & 1.49 & 0.88 & 0.94 & 0.17 & $<1$ & 3 & 889 & 0.9 & 0.37 & 5.1 & 25.7 & 13.0 & 31 & $<5$ & 64.4 & 10.9 & 0.10 & 0.0 & 13.1 \\
\hline V-06-5 O & 2006 & 48.52139 & -92.70499 & Ryan Lake & 9.50 & 2.43 & 11.70 & 1.13 & 1.40 & 1.59 & 0.37 & 1.03 & 0.08 & $<1$ & 2 & $\mid 1,740$ & 3.5 & 0.25 & 5.4 & $\mid 195.0$ & 53.6 & 16 & $<5$ & 86.5 & 6.7 & 0.07 & 0.0 & 92.0 \\
\hline V-06-6 O & 2006 & 48.52126 & -92.70521 & Ryan Lake & 24.30 & 4.64 & 4.12 & 1.11 & 1.78 & 0.60 & 1.72 & 0.27 & 0.15 & 2 & 2 & 856 & 1.1 & 0.25 & 1.4 & \begin{tabular}{|l|}
19.8 \\
\end{tabular} & 7.4 & 28 & $<5$ & 26.7 & 12.3 & 0.09 & 0.0 & 10.9 \\
\hline V-06-7 O & 2006 & 48.52016 & -92.70523 & Ryan Lake & 27.30 & 6.22 & 3.08 & 2.14 & 1.75 & 0.62 & 1.52 & 0.28 & 0.20 & $<1$ & 6 & 609 & 1.1 & 0.34 & 1.6 & 25.0 & 19.9 & 36 & $<5$ & 26.8 & 15.8 & 0.10 & 0.0 & 13.4 \\
\hline V-06-8 O & 2006 & 48.51816 & -92.70107 & Ryan Lake & 27.30 & 4.97 & 3.89 & 2.29 & 1.49 & 0.69 & 1.51 & 0.31 & 0.22 & $<1$ & 5 & $\mid 1,150$ & 1.1 & 0.41 & 2.3 & 26.7 & 13.4 & 32 & $<5$ & 26.8 & 14.2 & 0.14 & 0.0 & 13.7 \\
\hline V-06-9 O & 2006 & 48.51760 & -92.70024 & Ryan Lake & 6.30 & 1.72 & 13.50 & 1.11 & 1.81 & 2.28 & 0.39 & 1.49 & 0.07 & $<1$ & 3 & $\mid 1,300$ & 0.9 & 0.22 & 6.9 & 21.3 & 58.1 & 21 & 6 & $\mid 114.0$ & 5.2 & 0.08 & $<0.02$ & 12.6 \\
\hline V-06-10 O & 2006 & 48.51627 & -92.70162 & Ryan Lake & 13.50 & 5.51 & 9.05 & 2.62 & 1.62 & 1.59 & 1.12 & 0.64 & 0.23 & $<1$ & 6 & 748 & 1.1 & 0.34 & 4.6 & 24.5 & 19.1 & 57 & $<5$ & \begin{tabular}{|l|} 
\\
56.8
\end{tabular} & 14.1 & 0.09 & 0.0 & 13.1 \\
\hline V-06-11 O & 2006 & 48.51359 & -92.70248 & Ryan Lake & 13.30 & 3.71 & 11.40 & 1.32 & 1.54 & 1.23 & 0.71 & 0.75 & 0.13 & $<1$ & 3 & $\mid 1,350$ & 0.8 & 0.30 & 7.0 & 20.9 & 31.8 & 24 & $<5$ & 67.1 & 8.6 & 0.08 & 0.0 & 12.3 \\
\hline V-06-12 O & 2006 & 48.51781 & -92.69928 & Ryan Lake & 12.30 & 3.59 & 10.50 & 1.21 & 1.77 & 1.70 & 0.83 & 0.89 & 0.14 & $<1$ & 2 & $\mid 1,310$ & 1.0 & 0.31 & 5.3 & 22.8 & 36.5 & 24 & $<5$ & 74.5 & 9.4 & 0.08 & 0.0 & 12.7 \\
\hline V-06-13 O & 2006 & 48.51214 & -92.78509 & Brown Lake & 21.50 & 6.43 & 6.32 & 2.94 & 1.29 & 1.22 & 1.59 & 0.33 & 0.27 & $<1$ & 4 & $\mid 1,240$ & 1.2 & 0.46 & 4.5 & 28.9 & 27.1 & 65 & $<5$ & 47.5 & 15.8 & 0.10 & 0.0 & 15.3 \\
\hline V-06-14 O & 2006 & 48.51289 & -92.78571 & Brown Lake & 13.00 & 5.29 & 9.64 & 2.26 & 1.74 & 1.56 & 0.79 & 0.85 & 0.20 & $<1$ & 4 & 2,570 & 1.8 & 0.29 & 3.4 & $\mid 119.0$ & 31.1 & 42 & $<5$ & 95.3 & 11.6 & 0.08 & 0.0 & 66.5 \\
\hline V-06-15 O & 2006 & 48.51385 & -92.78703 & Brown Lake & 13.00 & 5.08 & 10.70 & 2.42 & 1.79 & 1.42 & 0.69 & 1.02 & 0.17 & $<1$ & 6 & $\mid 1,390$ & 1.0 & 0.47 & 4.1 & \begin{tabular}{|l|} 
\\
28.5 \\
\end{tabular} & 13.6 & 37 & $<5$ & 60.2 & 12.0 & 0.10 & 0.0 & 14.5 \\
\hline V-06-15 O dup & 2006 & 48.51383 & -92.78757 & Brown Lake & 12.30 & 4.10 & 9.88 & 2.19 & 1.88 & 1.38 & 0.82 & 0.95 & 0.17 & $<1$ & 5 & $\mid 1,310$ & 1.1 & 0.45 & 3.7 & 29.1 & 12.9 & 36 & $<5$ & 56.5 & 11.9 & 0.11 & 0.0 & 14.7 \\
\hline V-06-16 O & 2006 & 48.51383 & -92.78757 & Brown Lake & 10.30 & 3.85 & 12.10 & 1.83 & 1.97 & 1.67 & 0.68 & 1.08 & 0.13 & $<1$ & 5 & 1,150 & 0.8 & 0.39 & 4.8 & 27.9 & 15.2 & 26 & $<5$ & 57.5 & 9.1 & 0.12 & 0.0 & 14.7 \\
\hline
\end{tabular}


Appendix 3-O Horizon Chemistry, Ryan and Brown Lakes, Voyageurs National Park, MN

\begin{tabular}{|c|c|c|c|c|c|c|c|c|c|c|c|c|c|c|c|c|c|c|c|c|c|c|c|c|c|c|}
\hline Field no. & $\begin{array}{c}\text { Year } \\
\text { collected }\end{array}$ & atitude & Longitude & Watershed & $\begin{array}{l}\text { Ash } \\
(\%)\end{array}$ & $\begin{array}{c}\mathrm{Li} \\
(\mathrm{ppm})\end{array}$ & $\begin{array}{c}\mathrm{Mn} \\
(\mathrm{ppm}) \\
\end{array}$ & $\begin{array}{c}\text { Mo } \\
\text { (ppm) }\end{array}$ & $\begin{array}{c}\mathrm{Nb} \\
(\mathrm{ppm})\end{array}$ & $\begin{array}{c}\mathrm{Ni} \\
(\mathrm{ppm})\end{array}$ & $\begin{array}{c}\mathrm{P} \\
(\mathrm{ppm})\end{array}$ & $\begin{array}{c}\mathrm{Pb} \\
(\mathrm{ppm})\end{array}$ & $\begin{array}{c}\mathrm{Rb} \\
(\mathrm{ppm})\end{array}$ & $\begin{array}{c}\text { Sb } \\
(\mathrm{ppm})\end{array}$ & $\begin{array}{c}\mathrm{Sc} \\
(\mathrm{ppm})\end{array}$ & \begin{tabular}{|c|}
$\mathrm{Sn}$ \\
$(\mathrm{ppm})$
\end{tabular} & $\begin{array}{c}\mathrm{Sr} \\
(\mathrm{ppm})\end{array}$ & \begin{tabular}{|c|}
$\mathrm{Te}$ \\
$(\mathrm{ppm})$
\end{tabular} & $\begin{array}{c}\text { Th } \\
\text { (ppm) }\end{array}$ & $\begin{array}{c}\mathrm{Tl} \\
(\mathrm{ppm})\end{array}$ & $\begin{array}{c}U \\
(\mathrm{ppm})\end{array}$ & $\begin{array}{c}\text { V } \\
(\mathrm{ppm})\end{array}$ & $\begin{array}{c}\text { W } \\
(\mathrm{ppm})\end{array}$ & $\begin{array}{c}\mathrm{Y} \\
(\mathrm{ppm})\end{array}$ & \begin{tabular}{|c|}
$\mathrm{Zn}$ \\
$(\mathrm{ppm})$ \\
\end{tabular} & $\begin{array}{c}\mathrm{Se} \\
(\mathrm{ppm})\end{array}$ \\
\hline$-03-010$ & 2003 & 48.51360 & 2.70532 & Ryan Lake & 25.15 & 26 & 7,270 & 2.29 & 8.9 & 62.8 & 4,620 & 107.0 & 122.0 & 1.4 & 9.0 & 3.4 & 348 & 0.1 & 7.1 & 0.8 & 2.2 & 75 & 1.1 & 23.4 & 436 & 0.4 \\
\hline 3-010 dup & 03 & 51360 & 2.70532 & Ryan Lake & 20.60 & 16 & 9,670 & 1.89 & 4.1 & 140.0 & 10,235 & 46.9 & 116.0 & 1.33 & 8.5 & 1.6 & 859 & $<0.1$ & 6.5 & 0.6 & 3.6 & 43 & 0.6 & 69.3 & 673 & 0.3 \\
\hline 3-02O & 3 & 52164 & .70490 & Ryan Lake & 05 & 12 & 4,450 & 1.44 & 4.5 & 32.0 & 4,840 & 67.0 & 98.1 & 0.98 & 6.2 & 2.7 & 445 & $<0.1$ & 4.1 & 0.7 & 2.7 & 47 & 0.6 & 22.7 & 210 & 0.2 \\
\hline & & 52124 & .70567 & jan Lake & & 20 & 16,141 & & 1 & 81.3 & 8,700 & 109.0 & 179.0 & 1.66 & 9.4 & 3.1 & 433 & 0.1 & 10.0 & 1.4 & 4.8 & 73 & 1.0 & 32.0 & 420 & 0.4 \\
\hline $3-040$ & 03 & 51987 & 2.70518 & Ryan Lake & 00 & 17 & 13,913 & 2.32 & 7.7 & 38.7 & 9,320 & 134.0 & 136.0 & 1.41 & 5.2 & 3.0 & 409 & 0.1 & 5.3 & 1.2 & 5.0 & 53 & 1.0 & 10.4 & 916 & 0.3 \\
\hline V-03-05O & 2003 & 51889 & -92.70403 & Ryan Lake & 9.50 & 29 & 15,144 & 2.74 & 8.0 & 57.1 & 6,130 & 128.0 & 162.0 & 1.52 & 7.8 & 3.2 & 371 & 0.1 & 6.9 & 1.4 & 2.0 & 87 & 1.1 & 11.2 & 929 & 0.4 \\
\hline V-03-06O & & 51324 & -92.70163 & Ryan Lake & 16.30 & 25 & 6,280 & 2.24 & 7.7 & 52.3 & 4,720 & 73.9 & 132.0 & 1.05 & 6.8 & 2.7 & 331 & $<0.1$ & 5.6 & 0.8 & 1.5 & 80 & 0.9 & 8.0 & 443 & 0.5 \\
\hline 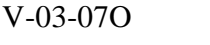 & & 1 & & jan Lake & & 10 & 13,308 & 1.78 & 3.3 & 39.2 & 12,022 & 53.9 & 106.0 & t & 4.0 & 1.9 & 666 & $<0.1$ & 2.7 & 1.0 & 0.9 & 39 & 0.6 & 7.3 & 968 & 0.2 \\
\hline & & 1711 & 0057 & Ryan Lake & & 18 & 11,235 & 32 & 6.3 & 37.7 & 6,640 & 115.0 & 176.0 & 1.34 & 6.3 & 2.8 & 597 & 0.1 & 5.0 & 0.8 & 1.5 & 64 & 0.8 & 9.6 & $\mid 1,350$ & 0.5 \\
\hline 090 & 03 & 51831 & 2.70119 & Ryan Lake & .80 & 12 & 9,090 & 2.07 & 4.3 & 28.4 & 8,190 & 82.0 & 115.0 & 1.16 & 5.1 & 2.3 & 650 & $<0.1$ & 3.6 & 0.6 & 1.1 & 46 & 0.7 & 7.9 & \begin{tabular}{|l|} 
\\
984
\end{tabular} & 0.2 \\
\hline V-03-10O & & 51792 & -92.69938 & Ryan Lake & 60 & 14 & 10,569 & 1.63 & 5.5 & 37.4 & 4,600 & 58.6 & 128.0 & 0.89 & 5.3 & 2.0 & 458 & $<0.1$ & 5.1 & 0.6 & 1.2 & 52 & 0.6 & 7.0 & 799 & 0.3 \\
\hline V-03- & & 1568 & 00 & Ryan & & 21 & 2,430 & 2.25 & 6.9 & 54.6 & 5,200 & 118.0 & 94.8 & 1.58 & 7.7 & 3.2 & 370 & $<0.1$ & 6.1 & 0.8 & 1.9 & 77 & 0.9 & 15.4 & 282 & 0.6 \\
\hline V-03-12O & 03 & 51580 & 2.70547 & Ryan Lake & 65 & 22 & 4,250 & 1.96 & 7.7 & 40.3 & 3,710 & 108.0 & 140.0 & 1. & 7.9 & 3.4 & 255 & $<0.1$ & 5.6 & 0.9 & 1.7 & 81 & 0.9 & 10.7 & 305 & 0.5 \\
\hline 03-130 & & 51373 & 2.70205 & Ryan Lake & 70 & 13 & 7,770 & 1.54 & 5.6 & 27.9 & 4,900 & 77.1 & 138.0 & 1.06 & 6.2 & 2.4 & 617 & $<0.1$ & 4.1 & 0.6 & 1.4 & 50 & 0.6 & 11.3 & 579 & 0.3 \\
\hline & & 1351 & 0242 & Ryan Lake & .55 & 16 & 7,450 & 1.74 & 5.0 & 31.4 & 4,760 & 84.8 & 147.0 & 0.93 & 5.3 & 2.1 & 437 & $<0.1$ & 4.2 & 0.7 & 1.2 & 45 & 0.6 & 8.3 & 266 & 0.4 \\
\hline & & 1373 & 8 & Brow & & 25 & 9,650 & 2.56 & 6.5 & 43.3 & 7,610 & 75.9 & 108.0 & 1.03 & 6.6 & 2.6 & 357 & $<0.1$ & 5.2 & 0.8 & 1.6 & 68 & 0.9 & 8.9 & 561 & 0.4 \\
\hline & & 1392 & 11 & Brow & 05 & 24 & 9,670 & 2.94 & 5.4 & 111.0 & 11,067 & 67.2 & 87.3 & 1.26 & 6.9 & 2.2 & 722 & $<0.1$ & 6.0 & 0.7 & 3.1 & 61 & 0.8 & 20.3 & 897 & 0.4 \\
\hline 03-160 dup & & 1392 & 11 & Brow & 17.95 & 12 & 10,392 & 2.58 & 3.6 & 44.7 & 20,240 & 48.5 & 82.4 & 0.83 & 3.6 & 1.6 & 777 & $<0.1$ & 3.5 & 0.4 & 1.0 & 39 & 0.5 & 6.3 & $|1,620|$ & 0.2 \\
\hline & & & & Brow & 0 & 27 & 8,040 & 1.95 & 7.2 & 38.5 & 6,690 & 52.9 & 108.0 & 0.77 & 7.1 & 2.2 & 346 & $<0.1$ & 6.2 & 0.5 & 1.7 & 70 & 0.9 & 8.7 & 517 & 0.3 \\
\hline $03-1$ & & 1218 & -92.7 & Brown & 0 & 25 & 6,830 & 1.94 & 8.0 & 61.4 & 4,830 & 102.0 & 128.0 & 1.28 & 8.2 & 2.8 & 358 & $<0.1$ & 6.7 & 0.9 & 1.9 & 73 & 1.0 & 20.1 & 454 & 0.4 \\
\hline 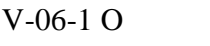 & 20 & 1472 & -92.70396 & Ryan Lake & .80 & 25 & 9,150 & 3.47 & 10.1 & 36.7 & 5,040 & 90.1 & 134.0 & 1.58 & 7.2 & 55.8 & 276 & 0.1 & 6.7 & 1.0 & 2.1 & 86 & 1.6 & 10.6 & 302 & 0.4 \\
\hline 6-2 O & & 1580 & -92.70547 & Ryan Lake & 50 & 20 & 4,640 & 2.00 & 6.3 & 51.2 & 6,880 & 82.8 & 92.2 & 1.43 & 7.7 & 34.3 & 377 & $<0.1$ & 37.6 & 0.8 & 2.0 & 64 & 0.9 & 15.4 & 284 & 0.3 \\
\hline & & & & & & 19 & 11,301 & 55 & 7 & 32.3 & 3,830 & 66.7 & 88.1 & 0.76 & 6.3 & 12.2 & 400 & $<0.1$ & 4.8 & 0.6 & 1.5 & 64 & 0.9 & 8.3 & 215 & $<0.2$ \\
\hline .06-4 & & & -92.7 & Ryan & & 17 & 12,813 & 2.20 & 5.6 & 33.8 & 9,880 & 58.7 & 114.0 & 1.26 & & 16.8 & 355 & $<0.1$ & 3.5 & 1.0 & 1.1 & & & 7.5 & 692 & 0.2 \\
\hline $6-50$ & & 2139 & 2.70499 & Ryan Lake & 9.5 & 10 & 8,970 & 1.63 & 3.8 & 98.7 & 10,962 & 34.6 & 89.0 & 0.97 & 5.3 & 6.8 & 810 & $<0.1$ & 3.7 & 0.4 & 1.8 & 26 & 0.5 & 40.5 & 620 & $<0.2$ \\
\hline V-06-6 O & & 2126 & -92.70521 & Ryan Lake & 24.30 & 8 & 5,010 & 1.08 & 4.2 & 14.4 & 3,060 & 29.5 & 82.2 & 0.46 & 5.5 & 1.5 & 323 & $<0.1$ & 2.7 & 0.6 & 1.0 & 37 & 0.5 & 6.5 & 167 & $<0.2$ \\
\hline & & & & & & 16 & 4,96 & 1.50 & 6.6 & 29.0 & 3,440 & 43.0 & 114.0 & 0.68 & 6.5 & 2.3 & 221 & $<0.1$ & 4.2 & 0.8 & 1.3 & 60 & 0.7 & 7.0 & 175 & 0.3 \\
\hline & & & & & & 17 & & & & 26.8 & & & 87.9 & & & & & $<0.1$ & & & 1.2 & & & & & 0.3 \\
\hline & & & & Ryan Lake & & 7 & 12,968 & 1.77 & 2.4 & 35.2 & 13,409 & 27.4 & 94.7 & 0.8 & 0.3 & 1.8 & 603 & $<0.1$ & 1.8 & 0.8 & 0.6 & 22 & 0.5 & 6.3 & 894 & $<0.2$ \\
\hline V-06-10 O & & 1627 & -92.70162 & Ryan Lake & 13.50 & 21 & 6,850 & 2.22 & 6.7 & 44.1 & 8,240 & 42.7 & 103.0 & 0.79 & 6.1 & 2.2 & 362 & $<0.1$ & 3.8 & 0.9 & 1.3 & 66 & 0.9 & 7.0 & 504 & 0.3 \\
\hline & & & & & & 9 & 8,920 & 1.73 & 4.8 & 20.8 & 9,080 & 43.6 & 90.2 & 0.79 & 4.5 & 2.5 & 720 & $<0.1$ & 2.8 & 0.4 & 0.9 & 32 & 0.5 & 6.3 & 689 & $<0.2$ \\
\hline & & & & & & 10 & & & & 21. & & & 98.4 & & 7.0 & 1.9 & 291 & $<0.1$ & 3.0 & 0.01 & 1.0 & & & 7.01 & & $<0.2$ \\
\hline & & & & & & 29 & & & 7 & & & & 79.0 & & & & 4 & .1 & 4.9 & & 1.0 & & 0 & 8.7 & & 0.2 \\
\hline $14 \mathrm{O}$ & & 1289 & -92.78571 & Brown Lake & 13.00 & 26 & 8,010 & 2.86 & 6.1 & 112.0 & 11,285 & 33.9 & 76.3 & 0.85 & 7.5 & 2.2 & 688 & $<0.1$ & 4.8 & 0.6 & 2.9 & 58 & 0.7 & 24.3 & 690 & 0.2 \\
\hline & & & -92.7870 & & 13. & 19 & 15,367 & 3.12 & 6.3 & 50.0 & 11,566 & 69.4 & 99.4 & 1.27 & 6.3 & 2.8 & 396 & $<0.1$ & 4.4 & 0.8 & 1.4 & 53 & 1.0 & 9.5 & 757 & 0.3 \\
\hline & & & & & & 20 & & 2.62 & 3.9 & 45.6 & 10,644 & 66.6 & 95.9 & 2.74 & & 2.9 & 383 & $<0.1$ & 4.6 & 0.8 & 1.4 & 54 & 0.9 & 9.1 & 714 & 0.3 \\
\hline $\mathrm{V}-06-16 \mathrm{O}$ & 2006 & 48.51383 & -92.78757 & Brown Lake & 0.30 & 14 & 12,248 & 2.39 & 4.7 & 35.9 & 10,736 & 68.2 & 104.0 & 1.08 & 4.9 & 2.4 & 540 & $<0.1$ & 3.4 & 0.7 & 1.2 & 43] & 0.7 & 8.1 & 392 & 0.2 \\
\hline
\end{tabular}


Click back to Contents

Appendix 4-A Horizon Chemistry, Shoepack Lake, Voyageurs National Park, MN

\begin{tabular}{|c|c|c|c|c|c|c|c|c|c|c|c|c|c|c|c|c|c|c|c|c|c|c|c|c|c|c|c|c|}
\hline Field no. & \begin{tabular}{|c|} 
Year \\
collected
\end{tabular} & Latitude & Longitude & Fire severity & $\begin{array}{c}\text { Al } \\
\text { (wt. \%) }\end{array}$ & $\begin{array}{c}\mathrm{Ca} \\
\text { (wt. \%) }\end{array}$ & $\begin{array}{c}\mathrm{Fe} \\
\text { (wt. \%) }\end{array}$ & $\begin{array}{c}\mathrm{K} \\
\text { (wt. \%) }\end{array}$ & $\begin{array}{c}\mathrm{Mg} \\
\text { (wt. \%) }\end{array}$ & $\begin{array}{c}\mathrm{Na} \\
\text { (wt. \%) }\end{array}$ & $\begin{array}{c}\text { S } \\
\text { (wt. \%) }\end{array}$ & $\begin{array}{c}\mathrm{Ti} \\
\text { (wt. \%) }\end{array}$ & $\begin{array}{c}\mathrm{Ag} \\
(\mathrm{ppm})\end{array}$ & $\begin{array}{c}\text { As } \\
\text { (ppm) }\end{array}$ & $\begin{array}{c}\mathrm{Ba} \\
(\mathrm{ppm})\end{array}$ & $\begin{array}{c}\mathrm{Be} \\
(\mathrm{ppm})\end{array}$ & $\begin{array}{c}\mathrm{Bi} \\
(\mathrm{ppm})\end{array}$ & $\begin{array}{c}\mathrm{Cd} \\
(\mathrm{ppm})\end{array}$ & \begin{tabular}{|c|}
$\mathrm{Ce}$ \\
$(\mathrm{ppm})$
\end{tabular} & $\begin{array}{c}\text { Co } \\
(\mathrm{ppm})\end{array}$ & $\begin{array}{c}\mathrm{Cr} \\
(\mathrm{ppm})\end{array}$ & $\begin{array}{c}\mathbf{C s}^{*} \\
(\mathrm{ppm})\end{array}$ & \begin{tabular}{|c|}
$\mathrm{Cu}$ \\
$(\mathrm{ppm})$ \\
\end{tabular} & $\begin{array}{c}\mathrm{Ga} \\
(\mathrm{ppm})\end{array}$ & \begin{tabular}{|c|}
$\mathrm{Hg}$ \\
$(\mathrm{ppm})$
\end{tabular} & \begin{tabular}{|c|} 
In \\
$(\mathrm{ppm})$
\end{tabular} & \begin{tabular}{|c|}
$\mathrm{La}$ \\
$(\mathrm{ppm})$
\end{tabular} & $\begin{array}{c}\mathrm{Li} \\
\text { (ppm) }\end{array}$ \\
\hline SP-04-1-1A & 2004 & 48.49490 & -92.88660 & unburned & \begin{tabular}{|l|}
5.73 \\
\end{tabular} & 1.40 & 1.56 & 1.53 & 0.45 & 2.03 & 0.02 & 0.15 & $<1$ & 2 & 447 & 1.3 & \begin{tabular}{l|l}
0.16 \\
\end{tabular} & \begin{tabular}{l|}
0.1 \\
\end{tabular} & \begin{tabular}{|l|}
18.2 \\
\end{tabular} & 5.0 & 33 & 1.72 & 8.0 & 13.40 & \begin{tabular}{|l|}
0.03 \\
\end{tabular} & $<0.02$ & 9.4 & 14 \\
\hline SP-04-1-2A & & 49495 & 2.88618 & low/medium & 4.94 & 1.29 & 25 & 32 & 39 & 1.69 & 0.04 & 12 & $<1$ & 2 & 426 & 1.1 & 0.15 & 0.2 & 14.8 & 4.7 & 19 & 2.04 & 5.1 & 11.50 & 0.05 & 0.02 & 7.7 & 11 \\
\hline SP-04-1-3A & & 49508 & 2.88588 & high & 5.19 & & & & & & 04 & & $<1$ & 3 & 486 & 1.1 & 0.19 & 0.3 & 15.9 & 5.9 & 35 & 2.84 & 8.3 & 12.60 & 03 & .02 & 8.5 & 14 \\
\hline SP-04-2-2A & & 49617 & - & low & 23 & & 24 & 20 & & 31 & 0.05 & 14 & $<1$ & 3 & 539 & 1.0 & 0.19 & 0.5 & 18.1 & 4.1 & 24 & 2.86 & 5.1 & 10.20 & .16 & .02 & 9.7 & \\
\hline SP-04-2-2 & 04 & 49617 & - & low $/ \mathrm{m}$ & 44 & 33 & 29 & 27 & 35 & 42 & 0.06 & 0.14 & $<1$ & 3 & 564 & 1.0 & 0.21 & 0.5 & 18.7 & 4.3 & 22 & 2.81 & 6.7 & 11.10 & 0.15 & 0.03 & 10.6 & \\
\hline SP-04-2-3A & & & 2.8 & high & 38 & & & & & & 0.05 & & $<1$ & 7 & 783 & 1.5 & 0.35 & 0.6 & 23.3 & 5.8 & 34 & 3.27 & 10.8 & 13.30 & 0.03 & 0.04 & 12.2 & 12 \\
\hline SP-04-3-1A & 04 & 19990 & 2.8 & high & 5.43 & & 34 & 45 & 39 & 1.99 & 0.02 & 0.13 & $<1$ & 2 & 434 & 1.3 & 0.14 & 0.2 & 14.9 & 5.0 & 31 & 1.55 & 5.0 & 13.10 & 0.04 & 0.02 & 8.1 & 11 \\
\hline SP-04-3-2A & & 49950 & -6 & lon & & & & & & 49 & 0.02 & 0.24 & $<1$ & 5 & 513 & 1.3 & 0.39 & 0.2 & 27.6 & 6.6 & 44 & 1.35 & 18.8 & 16.10 & $<0.02$ & 0.04 & 14.8 & 14 \\
\hline SP-04-3- & 04 & 49950 & -92. & unbt & 79 & & & & & 74 & 0.12 & 10 & $<1$ & 4 & 348 & 0.6 & 0.20 & 0.2 & 15.3 & 4.4 & 21 & 1.17 & 9.8 & 7.18 & 0.15 & 0.02 & 8.6 & \\
\hline SP-04-4- & & 9866 & -5 & & & & & & & & 0.11 & & $<1$ & 5 & 272 & 0.7 & 0.24 & 0.3 & 16.5 & 4.3 & 24 & 0.98 & 8.6 & 7.54 & 0.26 & .03 & 9.0 & \\
\hline & & & & & 48 & & & & & & 0.09 & & $<1$ & 3 & 185 & 0.7 & 0.20 & 0.4 & 14.2 & 2.2 & 23 & 3.10 & 6.1 & 6.19 & 0.19 & 0.02 & 7.7 & \\
\hline SP-04-4. & & & & high & & & & & & & 0.05 & 0.15 & $<1$ & 6 & 527 & 1.2 & 0.22 & 0.4 & 19.8 & 4.7 & 23 & 1.79 & 8.4 & 14.00 & 0.03 & 0.03 & 10.3 & 11 \\
\hline SP-04 & & & -6 & & & & & & & & 0.03 & & $<1$ & 2 & 426 & 1.1 & 0.09 & 0.1 & 12.2 & 3.2 & 17 & 1.52 & 3.0 & 11.30 & 0.06 & $<0.02$ & 6.4 & 8 \\
\hline SP-04-5 & & 0060 & -92. & & 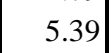 & & & & & & 0.02 & & $<1$ & 2 & 415 & 1.2 & 0.12 & 0.1 & 12.5 & 3.2 & 25 & 0.92 & 5.6 & 12.20 & 0.04 & $<0.02$ & 6.6 & \\
\hline SP-04 & & & $\mid-92$. & high & & & & & & & 0.07 & & $<1$ & 6 & 863 & 1.0 & 0.20 & 0.7 & 17.1 & 4.5 & 28 & 1.81 & 13.1 & 10.90 & 0.04 & \begin{tabular}{|l|l}
0.03 \\
\end{tabular} & 8.9 & 9 \\
\hline P-OS & & 42 & $\mid-5$ & & & & & & & & 0.09 & & $<1$ & 5 & 557 & 1.0 & 0.35 & 0.6 & 19.5 & 6.3 & 29 & 4.12 & 10.3 & 9.78 & 0.18 & 0.03 & 10.5 & 12 \\
\hline & & & & & & & & & & & 0. & & $<1$ & 4 & 547 & 1.0 & 0.26 & 0.2 & 19.0 & 2.8 & 23 & 2.19 & 7.9 & 11.40 & 0.09 & 0.02 & 10.6 & 8 \\
\hline & & 0180 & $\mid-92$. & his & & & & & & & 0.0 & & $<1$ & 5 & 660 & 1. & 0.2 & 0.5 & 20.4 & 3.4 & 29 & 2.98 & 13.4 & 13.00 & $<0.02$ & 0.03 & 11.2 & \\
\hline SP- 0 & & & & & & & & & & & 0 & & $<1$ & 3 & 294 & 0.8 & 0.16 & 0.2 & 15.7 & 2.6 & 20 & 1.52 & 6.1 & 9.12 & 0.14 & $<0.02$ & & 5 \\
\hline & & & & hig & & & & & & & 0 & & $<1$ & 4 & 544 & 1.5 & 0.22 & 0.3 & 17.9 & 4.4 & 22 & 2.50 & 6.0 & 12.40 & $<0.02$ & 0 & 9.6 & 10 \\
\hline & & & $\mid-92.8$ & & & & & & & & & & $<1$ & 3 & 386 & 0. & 0.2 & 0.2 & 14. & 6.5 & 35 & 2.44 & 6.5 & 9.58 & 0.12 & & 7.9 & 10 \\
\hline & & & & & & & & & & & & & $<1$ & 4 & 525 & 1.2 & 0.23 & 0.2 & 28.7 & 6.7 & 37 & 2.88 & 14.8 & 15.10 & 0.04 & .03 & 15.4 & 14 \\
\hline & & & & high & & & & & & & 0.04 & & $<1$ & 4 & 361 & 1.2 & 0.23 & 0.2 & 20.1 & 5.6 & 35 & 2.49 & 12.6 & 13.20 & 0.08 & 0.03 & 11.0 & 15 \\
\hline & & & & & & & & & & & & & $<1$ & 2 & 605 & $0 . \varepsilon$ & 0.1 & 5 & 18.6 & 5.9 & 11 & 1.35 & 5.1 & 25 & 14 & 02 & 11.1 & \\
\hline SP-04 & & 50080 & & & & & & & & & & & $<1$ & 2 & 588 & 0.7 & 0.1 & 0.5 & 18.0 & 5.8 & 1 . & 1.70 & 4.4 & 7.10 & 0.13 & .02 & 10.5 & \\
\hline & & & & & & & & & & & & & $<1$ & 3 & 586 & 1.0 & 0.24 & 0.4 & 25.0 & 12.4 & 27 & 2.30 & 7.1 & 10.20 & 0.11 & .03 & 13.7 & \\
\hline & & & & high & & & & & & & 0.05 & & $<1$ & 5 & 688 & 1.3 & 0.33 & 0.6 & 21.6 & 7.1 & 37 & 2.90 & 11.7 & 13.10 & 0.02 & .03 & 12.0 & 12 \\
\hline & & & & & & & & & & & & & $<1$ & 5 & 768 & & 0. & & 21. & 6.7 & 35 & 2.72 & 9.4 & & & & & 13 \\
\hline & & & -6 & & & & & & & & & & $<1$ & 4 & 503 & 0.9 & 0.2 & & 19. & 4.3 & 27 & 2.07 & 6.6 & 9.70 & 0.11 & & 10.5 & \\
\hline & & 50180 & 2.88450 & high & & & & & & & 0.02 & & $<1$ & 4 & 627 & 1.1 & 0.23 & 0.7 & 22.4 & 3.5 & 23 & 1.75 & 9.0 & 12.40 & $<0.02$ & 0.04 & 11.9 & \\
\hline & & & & high & & & & & & & 0.03 & & $<1$ & 4 & 650 & 1.3 & 0.25 & 0.7 & 21.3 & 3.5 & 24 & 2.04 & 8.6 & 12.60 & $<0.02$ & 0.03 & 11.4 & \\
\hline & & & & & & & & & & & & & $<1$ & 2 & 461 & 1.0 & 0.1 & 0.2 & 13.8 & 2.6 & 20 & 1.29 & 5.4 & 8.91 & 0.09 & & 7.6 & \\
\hline & & 8490 & & & & & & & & & & & $<1$ & 1 & 425 & & & & 12.8 & 2.7 & & 0.87 & 4.2 & & 0.07 & $<0.02$ & 7.1 & \\
\hline & & 9495 & -92.88618 & low/medium & & & & & & & 0.07 & & $<1$ & 2 & 466 & 1.5 & 0.14 & 0.4 & 15.0 & 3.4 & 18 & 1.84 & 6.3 & 7.66 & 0.09 & $<0.02$ & 8.4 & 6 \\
\hline & & 19508 & \begin{tabular}{|l|l} 
& -92.88588 \\
\end{tabular} & high & & & & & & & 0.05 & & $<1$ & 2 & 575 & 1.1 & 0.17 & 0.4 & 16.3 & 5.0 & 28 & 2.29 & 6.9 & 10.60 & 0.07 & 0.02 & 8.5 & 10 \\
\hline & & & & & & & & & & & & & $<1$ & & 682 & 0.9 & 0.2 & 0.6 & 25.5 & 15.8 & 30 & 2.51 & 6.1 & & & & 13.5 & \\
\hline & & & \begin{tabular}{|l|l} 
& -92.88976
\end{tabular} & & & & & & & & & & $<1$ & 3 & & & & & 17.6 & 4.1 & 26 & 2.92 & 6.9 & 9.74 & 0.15 & & 9.5 & \\
\hline$-05-2-3 A$ & & 49626 & \begin{tabular}{|l|l} 
& -92.88962
\end{tabular} & high & 5 & & & & & & 0.02 & 0.14 & $<1$ & 4 & 622 & 1.4 & 0.21 & 0.4 & 17.2 & 4.3 & 36 & 1.78 & 7.0 & 13.00 & $<0.02$ & 0.03 & 9.2 & 10 \\
\hline SP-05 & & .49990 & -92.88320 & high & 3.49 & 1.2 & 0.98 & & & & 0.06 & 0.08 & $<1$ & 2 & 347 & 0.8 & 0.11 & 0.3 & 13.2 & 3.3 & 23 & 1.34 & 4.5 & 7.76 & 0.09 & $<0.02$ & 7.4 & \\
\hline & & & & low $/ \mathrm{n}$ & .06 & .94 & 1.62 & & & & 0.04 & & $<1$ & 4 & 464 & 1.2 & 0.24 & 0.4 & 22.5 & 6.1 & 31 & 1.92 & 13.8 & 10.70 & 0.06 & 0.02 & 12.5 & 12 \\
\hline
\end{tabular}




\begin{tabular}{|c|c|c|c|c|c|c|c|c|c|c|c|c|c|c|c|c|c|c|c|c|c|c|c|c|c|c|c|c|}
\hline Field no. & $\begin{array}{c}\text { Year } \\
\text { collected }\end{array}$ & titude & Longitude & Fire severity & \begin{tabular}{|c|} 
Al \\
(wt. \%)
\end{tabular} & $\begin{array}{c}\mathrm{Ca} \\
\text { (wt. \%) }\end{array}$ & $\begin{array}{c}\mathrm{Fe} \\
\text { (wt. \%) }\end{array}$ & $\begin{array}{c}\mathrm{K} \\
\text { (wt. \%) }\end{array}$ & \begin{tabular}{|c|}
$\mathrm{Mg}$ \\
(wt. \%)
\end{tabular} & $\begin{array}{c}\mathrm{Na} \\
\text { (wt. \%) }\end{array}$ & $\begin{array}{c}\mathrm{S} \\
\text { (wt. \%) }\end{array}$ & \begin{tabular}{|c|}
$\mathrm{Ti}$ \\
(wt. \%)
\end{tabular} & $\begin{array}{c}\mathrm{Ag} \\
(\mathrm{ppm})\end{array}$ & \begin{tabular}{|c|} 
As \\
(ppm)
\end{tabular} & \begin{tabular}{|c|}
$\mathrm{Ba}$ \\
$(\mathrm{ppm})$ \\
\end{tabular} & \begin{tabular}{|c|}
$\mathrm{Be}$ \\
$(\mathrm{ppm})$
\end{tabular} & $\begin{array}{c}\mathrm{Bi} \\
(\mathrm{ppm})\end{array}$ & $\begin{array}{c}\mathrm{Cd} \\
(\mathrm{ppm})\end{array}$ & \begin{tabular}{|c|}
$\mathrm{Ce}$ \\
$(\mathrm{ppm})$
\end{tabular} & $\begin{array}{c}\text { Co } \\
(\mathrm{ppm})\end{array}$ & \begin{tabular}{|c|}
$\mathrm{Cr}$ \\
$(\mathrm{ppm})$
\end{tabular} & \begin{tabular}{|c|}
$\begin{array}{c}\mathrm{CS}^{*} \\
(\mathrm{ppm})\end{array}$ \\
\end{tabular} & \begin{tabular}{|c|}
$\mathrm{Cu}$ \\
$(\mathrm{ppm})$
\end{tabular} & \begin{tabular}{|c|} 
Ga \\
$(\mathrm{ppm})$
\end{tabular} & \begin{tabular}{|c|}
$\mathrm{Hg}$ \\
$(\mathrm{ppm})$
\end{tabular} & \begin{tabular}{|c|} 
In \\
$(\mathrm{ppm})$
\end{tabular} & \begin{tabular}{|c|}
$\begin{array}{c}\text { La } \\
(\mathrm{ppm})\end{array}$ \\
\end{tabular} & $\begin{array}{c}\mathrm{Li} \\
(\mathrm{pppm})\end{array}$ \\
\hline SP-05-4-1A & 005 & 49866 & 2.89069 & unburned & 1.16 & 0.44 & 0.56 & 0.30 & \begin{tabular}{|l|}
0.13 \\
\end{tabular} & 0.18 & 0.13 & \begin{tabular}{|l|}
0.05 \\
\end{tabular} & $<1$ & 3 & 164 & \begin{tabular}{|l|}
0.4 \\
\end{tabular} & \begin{tabular}{|l|}
0.14 \\
\end{tabular} & 0.5 & 9.5 & 3.4 & 11 & \begin{tabular}{|l|}
0.30 \\
\end{tabular} & \begin{tabular}{|l|}
5.4 \\
\end{tabular} & \begin{tabular}{|l|}
2.80 \\
\end{tabular} & \begin{tabular}{|l|}
0.26 \\
\end{tabular} & $<0.02$ & \begin{tabular}{|l|}
5.6 \\
\end{tabular} & \\
\hline SP-05-4-1A d & & 9866 & 89069 & & 1.38 & 0.46 & 61 & 36 & .15 & 0.23 & 0.15 & 0.05 & $<1$ & 4 & 188 & 0.6 & 0.16 & 0.5 & 11.3 & 3.8 & 12 & .49 & 6.9 & 3.43 & 0.26 & $<0.02$ & 6.3 & \\
\hline SP-05-4-2A & 05 & 49850 & 2.89060 & low/medium & 2.22 & 0.41 & 0.84 & 0.49 & 17 & 0.45 & 0.06 & 0.08 & $<1$ & 4 & 201 & 0.7 & 0.26 & 0.3 & 13.7 & 1.5 & 17 & 0.40 & 6.1 & 6.45 & 0.18 & $<0.02$ & 7.4 & \\
\hline SP-05-4-3A & & 30 & 2.03 & high & 4.90 & 89 & 48 & & 36 & 67 & 03 & 13 & $<1$ & 6 & 573 & 1.3 & 0.23 & 0.7 & 19.5 & 5.0 & 29 & 1.75 & 9.9 & $\mid 11.70$ & $<0.02$ & 0.03 & 9.9 & \\
\hline ?-05-5-1A & & & 88 & unburı & 3.96 & & & & & & & & $<1$ & 2 & 513 & 1.0 & 0.29 & .4 & 13.4 & 4.2 & 22 & & 4.4 & \begin{tabular}{|l|} 
\\
\end{tabular} & 0.11 & $<0.02$ & 6.8 & \\
\hline P-05-5-2A & & & 289 & low $/ \mathrm{m}$ & 4.22 & 41 & 02 & & & 56 & 04 & 09 & $<1$ & 2 & 477 & 1.0 & 0.12 & 0.2 & 11.8 & 3.2 & 22 & 1.58 & 3.4 & 9.64 & 0.08 & $<0.02$ & 6.3 & \\
\hline SP-05-5-3A & & 50060 & -5 & high & 4.00 & & & & & 36 & .05 & 0.10 & $<1$ & 4 & 666 & 1.1 & 0.21 & 0.6 & 16.6 & 3.4 & 24 & 1.73 & 6.8 & 9.48 & 0.11 & 0.03 & 8.9 & 6 \\
\hline$-05-6$ & & 12 & 0 & unbs & 86 & & & & & & 07 & & $<1$ & 4 & 512 & 1.8 & 0.35 & 0.4 & 20.1 & 5.8 & 33 & 3.49 & 10.4 & $\mid 10.80$ & 0.17 & 0.03 & 10.4 & 13 \\
\hline SP-05-6- & & 0170 & & & 48 & & & & & & & & $<1$ & 4 & 598 & 0.5 & 0.14 & 0.4 & 15.9 & 3.4 & 18 & & 9.3 & 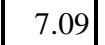 & 0.06 & t & & 6 \\
\hline SP-05-6-3A & & 50180 & 2.8 & high & 4.25 & & 00 & 25 & 24 & .51 & 02 & 0.12 & $<1$ & 3 & 511 & 1.0 & 0.17 & 0.3 & 15.3 & 2.6 & 22 & 0.69 & 7.4 & $\mid 11.00$ & $<0.02$ & 0.02 & 8.2 & 6 \\
\hline$-05-7$ & & & & unb & 1.21 & & & & & 24 & 14 & 0.04 & $<1$ & 4 & 229 & 0.4 & 0.11 & 0.2 & 9.1 & 1.6 & 13 & .84 & 5.9 & 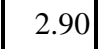 & 0.38 & $<0.02$ & 5.0 & 3 \\
\hline & & & & & 49 & & & & & & & & $<1$ & 5 & 355 & 1.2 & 0.28 & 0.4 & 23.0 & 9.3 & 22 & 2.35 & 12.5 & 5.54 & 0.32 & 0.03 & 13.5 & 8 \\
\hline SP-05-7-3A & & 50480 & -92.89560 & high & 4.90 & & & & & & & & $<1$ & 3 & 556 & 1.2 & 0.24 & 0.3 & 20.1 & 5.0 & 31 & & 8.6 & 11.60 & 0.02 & .03 & & 10 \\
\hline SP-05-8-1A & & 50166 & -92.87214 & unburn & .04 & & & & & 84 & 88 & 09 & $<1$ & 3 & 409 & 0.7 & 0.20 & 0.4 & 13.9 & 5.8 & 22 & 1.44 & 5.7 & 7.42 & 0.15 & .02 & & 8 \\
\hline SP-05-8-2 & & 50170 & & low/ & 4.01 & & & & & 0.61 & 0.05 & 0.15 & $<1$ & 3 & 471 & 1.4 & 0.26 & 0.3 & 33.3 & 5.8 & 33 & 1.61 & 14.9 & 11.10 & 0.09 & 0.03 & 19.0 & 11 \\
\hline SP-0 & & & & hig & & & & & & & & & $<1$ & 4 & 320 & 1.1 & 0.22 & .5 & 15.5 & 5.0 & 33 & & 10.6 & 10.20 & 0.10 & .0 & 9.5 & 12 \\
\hline SP-05-9-1A & & 50080 & $-\underline{s}$ & & & & & & & & & & $<1$ & 2 & 696 & 0.9 & 0.18 & 7 & 14.3 & 7.2 & 25 & 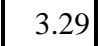 & 5.9 & 8.59 & 0.12 &. .6 & 8.1 . & 7 \\
\hline SP-0 & & 0097 & -5 & & 56 & & & & & & & & $<1$ & 2 & 592 & 0.8 & 0.18 & 0.5 & 16.1 & 12.5 & 22 & 32 & 9.5 & 6.83 & 0.14 & .0 & 9.1 & 6 \\
\hline & & 0130 & & high & & & & & & & 05 & & $<1$ & 4 & 940 & 1.0 & 0.32 & 1.2 & 21.5 & 9.8 & 32 & .04 & 15.4 & 10.70 & 0.05 & 0.04 & 11.7 & 10 \\
\hline SP-05-1 & & 0180 & & high & 41 & & & & & & & & $<1$ & 3 & 557 & 1.2 & 0.26 & 0.5 & 16.7 & 2.7 & 21 & 0.68 & 7.1 & $\mid 10.70$ & 0.02 & .02 & 9.1 & 5 \\
\hline & & & & hig & 60 & & & & & & & & $<1$ & 3 & 585 & 1.1 & 0.21 & 0.5 & 18.9 & 3.4 & 26 & 1.42 & 8.8 & $\mid 11.40$ & $<0.02$ & 0.03 & 10.2 & 6 \\
\hline & & & 2. & & & & & & & & & & $<1$ & 4 & 492 & 0.9 & 0.1 & 3 & $12 . c$ & 4.7 & 32 & $<5$ & 11.0 & $\mid 11.10$ & 0.03 & 0.0 & 7.2 & 10 \\
\hline SP-06 1-2A & & 9495 & & & & & & & & & & & $<1$ & 2 & 445 & 0.8 & 0.12 & 0.4 & 13. & 4.5 & 3 & $<5$ & 10.8 & \begin{tabular}{|l|} 
\\
\end{tabular} & 0.04 & $<0.02$ & 7.2 & 10 \\
\hline SP-06 1-3A & & & & hig & & & & & & & & & $<1$ & 3 & 461 & 1.0 & 0.07 & & 14.9 & 3.1 & 25 & $<5$ & 6.9 & 11.10 & 0.03 & $<0.02$ & 8.6 & 6 \\
\hline & & & & & & & & & & & & & $<1$ & 6 & 577 & 0.8 & 0.16 & & 20.0 & 11.4 & 32 & $<5$ & 12.5 & \begin{tabular}{|l|} 
\\
\end{tabular} & 0.20 & 0.03 & 11.2 & 8 \\
\hline & & & & & & & & & & & & & $<1$ & 5 & 80 & & 0.2 & & 20. & & & $<5$ & 11.1 & 10.80 & 0.10 & & 11 & 12 \\
\hline $062-$ & & 9626 & & his & & & & & & & & & $<1$ & 7 & 547 & 1.2 & 0.16 & & 17.5 & 5.5 & 44 & $<5$ & 10.1 & $\mid 14.20$ & $<0.02$ & 0.0 & 9.9 & 13 \\
\hline & & & & hig & & & & & & & & & $<1$ & 4 & 73 & 1.1 & 0.08 & & 15.4 & 5. & 35 & $<5$ & 9.1 & 11.00 & 0.04 & $<0.02$ & 8.3 & 10 \\
\hline & & & & & & & & & & & & & $<1$ & 4 & 441 & 1.0 & 0.1 & & 20. & 5. & 34 & $<5$ & 14.9 & 11.50 & 0.05 & 0.02 & 11.8 & 10 \\
\hline & & & & & & & & & & & & & $<1$ & 4 & & & 0.1 & & & & & $<5$ & 12.5 & 10.80 & & & & \\
\hline SI & & & & & & & & & & & & & $<1$ & 4 & & 0.8 & 0.26 & & 17.9 & 6.2 & 37 & $<5$ & 15.5 & 10.80 & 0.07 & & 9.7 & 10 \\
\hline -06 4-1A & & 9866 & -92.89 & & 4.62 & & & & & & & & $<1$ & 4 & 438 & 0.9 & 0.13 & 0.4 & 22.0 & 18.2 & 35 & $<5$ & 11.6 & 11.10 & 0.11 & 0.03 & 12.4 & 10 \\
\hline & & & & & & & & & & & & & $<1$ & 6 & $23 s$ & 1.2 & 0.26 & & 17.4 & 2.7 & 19 & $<5$ & 11.1 & \begin{tabular}{|l|} 
\\
\end{tabular} & 0.13 & 0.03 & 9.9 & \\
\hline & & & & hig & & & & & & & & & $<1$ & 5 & & & 0.0 & & & & & & 11.2 & $\mid 13.40$ & $<0.02$ & & & \\
\hline & & & & & & & & & & & & & $<1$ & 4 & & 0.9 & 0.24 & & & & & $<5$ & 6.9 & & 0.13 & & & 11 \\
\hline$-065-2 A$ & & 0060 & -92.88860 & low/medium & 5.66 & & & & & 97 & 02 & 0.13 & $<1$ & 3 & 430 & 1.1 & $<0.04$ & 0.1 & 11.4 & 3.8 & 30 & $<5$ & 5.9 & $\mid 12.50$ & 0.03 & $<0.02$ & 6.1 & \\
\hline & & & & high & & & & & & & & & $<1$ & & & 1. & 0.15 & & 13. & 4.4 & 25 & $<5$ & 8.1 & 8.09 & 0.19 & & 7.3 & \\
\hline & & & & & & & & & & & & & $<1$ & & & 1.2 & 0.12 & & 13. & 4.6 & 29 & $<5$ & 8.9 & 10.10 & 0.09 & $<0.02$ & 7.7 & \\
\hline & & & & & & & & & & & & & $<1$ & & & & 0.28 & & 19.9 & & & $<5$ & 16.5 & 11.10 & 0.01 & & 11.0 & 0 \\
\hline SP-06 6-3A & & 50180 & -92.89100 & high & 5.00 & 1.41 & 1. & & & 1.54 & 0.02 & 0.17 & $<1$ & 5 & 542 & 1.1 & 0.13 & 0.4 & 16.5 & 3.1 & 27 & $<5$ & 12.9 & 11.90 & $<0.02$ & 0.03 & 9.2 & \\
\hline
\end{tabular}


Appendix 4-A Horizon Chemistry, Shoepack Lake, Voyageurs National Park, MN

\begin{tabular}{|c|c|c|c|c|c|c|c|c|c|c|c|c|c|c|c|c|c|c|c|c|c|c|c|c|c|c|c|c|}
\hline Field no. & $\begin{array}{c}\text { Year } \\
\text { collected }\end{array}$ & Latitude & Longitude & Fire severity & $\begin{array}{c}\mathrm{Al} \\
\text { (wt. \%) }\end{array}$ & $\begin{array}{c}\mathrm{Ca} \\
\text { (wt. \%) }\end{array}$ & $\begin{array}{c}\mathrm{Fe} \\
\text { (wt. \%) }\end{array}$ & $\begin{array}{c}\mathrm{K} \\
\text { (wt. \%) }\end{array}$ & \begin{tabular}{c|}
$\mathrm{Mg}$ \\
(wt. \%)
\end{tabular} & $\begin{array}{c}\mathrm{Na} \\
\text { (wt. \%) }\end{array}$ & \begin{tabular}{|c|}
$\mathrm{S}$ \\
(wt. \%)
\end{tabular} & \begin{tabular}{c|}
$\mathrm{Ti}$ \\
(wt. \%)
\end{tabular} & $\begin{array}{c}\mathrm{Ag} \\
(\mathrm{ppm})\end{array}$ & $\begin{array}{c}\text { As } \\
\text { (ppm) }\end{array}$ & $\begin{array}{c}\mathrm{Ba} \\
\text { (ppm) }\end{array}$ & $\begin{array}{c}\mathrm{Be} \\
(\mathrm{ppm})\end{array}$ & $\begin{array}{c}\mathrm{Bi} \\
(\mathrm{ppm})\end{array}$ & $\begin{array}{c}\mathrm{Cd} \\
(\mathrm{ppm})\end{array}$ & $\begin{array}{c}\mathrm{Ce} \\
(\mathrm{ppm})\end{array}$ & $\begin{array}{c}\text { Co } \\
(\mathrm{ppm})\end{array}$ & $\begin{array}{c}\mathrm{Cr} \\
(\mathrm{ppm})\end{array}$ & $\begin{array}{c}\mathrm{Cs}^{\star} \\
(\mathrm{ppm})\end{array}$ & $\begin{array}{c}\mathrm{Cu} \\
\text { (ppm) }\end{array}$ & \begin{tabular}{c|}
$\mathrm{Ga}$ \\
$(\mathrm{ppm})$
\end{tabular} & $\begin{array}{c}\mathrm{Hg} \\
(\mathrm{ppm})\end{array}$ & $\begin{array}{c}\text { In } \\
(\mathrm{ppm})\end{array}$ & $\begin{array}{c}\mathrm{La} \\
(\mathrm{ppm}) \\
\end{array}$ & $\begin{array}{c}\mathrm{Li} \\
(\mathrm{ppm})\end{array}$ \\
\hline $67-1 \mathrm{~A}$ & 2006 & 48.50540 & -92.89810 & unburned & 2.50 & 0.40 & 1.01 & \begin{tabular}{|l|}
0.57 \\
\end{tabular} & $\begin{array}{l}0.18 \\
\end{array}$ & 0.44 & $\begin{array}{l}0.12 \\
\end{array}$ & $\begin{array}{l}0.09 \\
\end{array}$ & $<1$ & 6 & 234 & 0.7 & 0.08 & 0.3 & $\begin{array}{ll}17.9 \\
\end{array}$ & 3.5 & 22 & $<5$ & 9.5 & \begin{tabular}{l|l}
5.99 \\
\end{tabular} & 0.24 & 0.02 & 10.8 & \\
\hline $67-2 \mathrm{~A}$ & 20 & & & & & & 1.47 & 0.69 & 26 & 61 & 0.09 & 0.12 & $<1$ & 9 & 359 & 1.4 & 0.21 & 0.4 & 23.5 & 11.8 & 29 & $<5$ & 12.3 & 7.67 & 0.24 & 0.03 & 13.7 & \\
\hline $67-3 A$ & & & & high & & & & 1. & & & 0.03 & 0.14 & $<1$ & 6 & 594 & 1.3 & 0.20 & 0.6 & 20.0 & 6.3 & 30 & $<5$ & 18.6 & 11.80 & 0.02 & .03 & 10.7 & \\
\hline $68-1 \mathrm{~A}$ & & & & & & & 4 & & & & & 0.17 & $<1$ & & 499 & 1.1 & 0.19 & 0.4 & 18. & 8.5 & 30 & $<5$ & 11.4 & 12.30 & .04 & & 9.6 & \\
\hline $68-2 A$ & 2006 & & & & 4.38 & & 1.74 & 1.00 & & 70 & 0.07 & 0.19 & $<1$ & 6 & 472 & 1.2 & 0.23 & 0.6 & 31.6 & 9.4 & 38 & $<5$ & 22.8 & 11.10 & 0.09 & 04 & 18.6 & \\
\hline $68-$ & & & & & 4.18 & 60 & 1.66 & 0.97 & 0. & 0.68 & 0.07 & 0.18 & $<1$ & 7 & 457 & 1.5 & 0.23 & 0.6 & 32.8 & 9.2 & 36 & $<5$ & 22.2 & 10.30 & 0.10 & 0.03 & 18.5 & \\
\hline $668-3$ & & & & high & 5.4 & $19 \mathrm{l}$ & 1.47 & 1.25 & & 1.45 & 0.03 & 0.23 & $<1$ & 6 & 465 & 1.2 & 0.21 & 0.3 & 21.8 & 3.7 & 36 & $<5$ & 33.4 & 14.50 & 0.04 & .03 & 11.9 & \\
\hline 6 9-1A & 2006 & & & & & 28 & 0.81 & 0.97 & & 0.93 & 0.09 & 0.10 & $<1$ & 4 & 654 & 0.9 & 0.12 & 0.7 & 16.2 & 7.7 & 22 & $<5$ & 8.8 & 7.70 & 0.13 & 0.02 & 9.6 & \\
\hline 6 9-2A & 2006 & & & low/medium & 3.43 & 1.16 & 1.04 & 0.88 & 0.32 & 0.81 & 0.10 & 0.12 & $<1$ & 6 & 623 & 0.9 & 0.16 & 0.6 & 18.4 & 18.4 & 27 & $<5$ & 14.1 & 7.99 & 0.15 & 0.03 & 10.2 & \\
\hline $69-3$ & & & & high & 4.81 & 1.20 & 1.34 & 1.15 & & 1.33 & 0.03 & 0.17 & $<1$ & 5 & 586 & 1.3 & 0.26 & 0.8 & 18.6 & 6.6 & 33 & $<5$ & 13.0 & 11.80 & 0.05 & 0.03 & 10.2 & \\
\hline 010 & & & & & & & & & & & & & $<1$ & 0 & 641 & 0.9 & 0.19 & & 20 & . & & $<5$ & 11.8 & 11.60 & .13 & & 10.7 & \\
\hline & & & & low/me & 5.21 & 43 & 1.50 & 1.29 & & 1.37 & 0.04 & 0.16 & $<1$ & & 610 & 1.2 & 0.22 & 0.9 & 20.6 & 7.7 & 35 & $<5$ & 10.8 & 11.10 & 0.11 & 0.03 & 11.3 & \\
\hline 6 10-3A & 2006 & 48.50180 & -92.88450 & high & 4.90 & 1.22 & 1.02 & 1.36 & 0.23 & 1.48 & 0.02 & 0.15 & $<1$ & & 555 & 1.2 & 0.15 & 0.8 & 17.0 & 3.3 & 24 & $<5$ & 10.1 & 10.20 & 0.03 & 0.03 & 9.4 & \\
\hline
\end{tabular}

*Because of analytical laboratory issues, Cs detection limits in 2004 and 2005 were 0.05 ppm and 5 ppm in 2006. 


\begin{tabular}{|c|c|c|c|c|c|c|c|c|c|c|c|c|c|c|c|c|c|c|c|c|c|c|c|c|c|}
\hline Field no. & $\begin{array}{c}\text { Year } \\
\text { collected }\end{array}$ & atitude & ngitude & Fire severity & \begin{tabular}{|c|}
$\mathrm{Mn}$ \\
$(\mathrm{ppm})$
\end{tabular} & $\begin{array}{c}\text { Mo } \\
(\mathrm{ppm})\end{array}$ & \begin{tabular}{|c|}
$\begin{array}{c}\mathrm{Nb} \\
(\mathrm{ppm})\end{array}$ \\
\end{tabular} & $\begin{array}{c}\mathrm{Ni} \\
(\mathrm{ppm})\end{array}$ & $\begin{array}{c}P \\
(\mathrm{ppm})\end{array}$ & \begin{tabular}{|c|}
$\mathrm{Pb}$ \\
(ppm)
\end{tabular} & \begin{tabular}{|c|}
$\begin{array}{c}\mathrm{Rb} \\
(\mathrm{ppm})\end{array}$ \\
\end{tabular} & $\begin{array}{c}\mathrm{Sb} \\
(\mathrm{ppm})\end{array}$ & $\begin{array}{c}\mathrm{Sc} \\
(\mathrm{ppm})\end{array}$ & \begin{tabular}{|c|}
$\mathrm{Sn}$ \\
$(\mathrm{ppm})$
\end{tabular} & \begin{tabular}{|c|}
$\mathrm{Sr}$ \\
$(\mathrm{ppm})$
\end{tabular} & $\begin{array}{c}\mathrm{Te} \\
(\mathrm{ppm})\end{array}$ & $\begin{array}{c}\text { Th } \\
(\mathrm{ppm})\end{array}$ & $\begin{array}{c}\mathrm{TI} \\
(\mathrm{ppm})\end{array}$ & \begin{tabular}{|c|}
$U$ \\
$(\mathrm{ppm})$ \\
\end{tabular} & $\begin{array}{c}V \\
(\mathrm{ppm})\end{array}$ & $\begin{array}{c}\mathrm{W} \\
(\mathrm{ppm})\end{array}$ & $\begin{array}{c}\mathrm{Y} \\
(\mathrm{ppm})\end{array}$ & \begin{tabular}{|c|}
$\mathrm{Zn}$ \\
$(\mathrm{ppm})$ \\
\end{tabular} & \begin{tabular}{|c|} 
Se \\
$(\mathrm{ppm})$
\end{tabular} & $\begin{array}{l}\text { Total C } \\
\text { (wt. \%) }\end{array}$ \\
\hline -04-1-1A & 2004 & 48.49490 & 2.88660 & unburned & 406 & 0.28 & \begin{tabular}{|l|}
4.8 \\
\end{tabular} & 13.8 & 460 & 21.6 & \begin{tabular}{|l|}
77.3 \\
\end{tabular} & 0.20 & 5.1 & 1.5 & 264 & $<0.1$ & 4.4 & 0.3 & \begin{tabular}{|l|}
1.1 \\
\end{tabular} & 42 & 0.4 & 5.8 & 34 & \begin{tabular}{|l|}
0.2 \\
\end{tabular} & 3.98 \\
\hline$-04-1-2 \mathrm{~A}$ & & 49495 & .88618 & low/m & 578 & 0.24 & 3.6 & 0.9 & 570 & 25.8 & 9.1 & 24 & 4.6 & 1.4 & 230 & $<0.1$ & 2.6 & 0.2 & 0.9 & 31 & 0.3 & 4.6 & 37 & 0.3 & 8.48 \\
\hline $04-1-3 \mathrm{~A}$ & 04 & 9508 & & high & 727 & 0.58 & 4.5 & 15.5 & 790 & 3.1 & 77.5 & .36 & 5.0 & 1.8 & 246 & $<0.1$ & 3.0 & 0.3 & 1.0 & 49 & 0.4 & 5.2 & 58 & 0.3 & 8.44 \\
\hline $04-2-1 \mathrm{~A}$ & & 49599 & & unburn & 2960 & 0.59 & 3.3 & 8.6 & 900 & 52.2 & 0.0 & 61 & 4.0 & 1.5 & 171 & $<0.1$ & 3.0 & 0.4 & 0.9 & 31 & 0.4 & 5.5 & 52 & 0.5 & 23.60 \\
\hline P-04-2-2A & 04 & .49617 & 776 & low $/ \mathrm{m}$ & 1350 & 0.44 & 4.1 & 8.9 & 630 & 38.2 & 70.2 & 0.45 & 4.7 & 2.8 & 213 & $<0.1$ & 3.3 & 0.4 & 1.0 & 34 & 0.7 & 6.2 & 61 & 0.4 & 13.30 \\
\hline $0+2$ & & & & & 370 & 0.47 & 4.2 & 10.2 & 630 & 9.5 & 6.2 & 49 & 4.8 & 1.2 & 222 & $<0.1$ & 3.2 & 0.5 & 1.0 & 37 & 0.4 & 5.6 & 63 & 0.4 & 12.10 \\
\hline$-04-2-3 A$ & 2 & 9626 & 2.88962 & high & 764 & 0.53 & 4.7 & 16.7 & 1,200 & 60.0 & 81.3 & 1.01 & 5.8 & 3.4 & 282 & $<0.1$ & 3.9 & 0.6 & 1.3 & 47 & 0.7 & 7.4 & 64 & 0.8 & 5.52 \\
\hline $04-3-1 \mathrm{~A}$ & & 19990 & 8320 & high & 388 & 0.25 & 3.7 & 12.0 & 330 & 24.4 & 73.1 & 0.21 & 5.0 & 1.4 & 243 & $<0.1$ & 3.1 & 0.4 & 0.9 & 37 & 0.5 & 5.1 & 27 & $<0.2$ & 4.65 \\
\hline $04-3-2$ & & 49950 & 380 & low $/ 1$ & 254 & 1.00 & 7.4 & 16.8 & 320 & 38.9 & 58.6 & 0.46 & 6.2 & 3.0 & 202 & $<0.1$ & 5.0 & 0.4 & 6.3 & 75 & 0.7 & 6.7 & 50 & 0.4 & 3.69 \\
\hline $3-$ & & & & unb & 411 & 69 & 3.3 & 12.3 & 620 & 0.1 & 35.6 & & 3.3 & 2.3 & 128 & $<0.1$ & 2.6 & 0.2 & 4.0 & 31 & 0.4 & 4.0 & 37 & 0.6 & 26.10 \\
\hline A & & & & & 177 & 0.88 & 3.9 & 10.8 & 840 & 40.0 & 29.5 & & 3.3 & 2.1 & 95 & $<0.1$ & 2.8 & 0.2 & 1.1 & 37 & 0.5 & 3.9 & 41 & 0.5 & 27.80 \\
\hline $1-4-2$ & & 9850 & & & 132 & 0.43 & 2.4 & 6.1 & 830 & 33.5 & 29.3 & & 3.3 & 1.6 & 126 & $<0.1$ & 2.9 & 0.2 & 3.5 & 23 & 0.4 & 4.0 & 35 & 0.5 & 31.40 \\
\hline & & & & high & 465 & 0.58 & 4.3 & 14.1 & 740 & 38.3 & 77.5 & 0 & 5.3 & 3.0 & 250 & $<0.1$ & 3.9 & 0.4 & 1.2 & 48 & 0.4 & 5.6 & 69 & 0.5 & 3.08 \\
\hline & & & & & 434 & 0. & 3.4 & 7.8 & 330 & 19.5 & 2.5 & & 4.3 & 1.5 & 237 & $<0.1$ & 2.3 & 0.3 & 0.8 & 29 & 0.3 & 4.5 & 31 & $<0.2$ & 6.24 \\
\hline & & & & & 286 & 0.22 & 3.3 & 8.5 & 250 & 20.2 & .2 & & 4.6 & 1.2 & 251 & $<0.1$ & 3.5 & 0.3 & 0.9 & 34 & 0.3 & 4.9 & 23 & $<0.2$ & 4.45 \\
\hline $4-5-3$ & & 0060 & & high & 1050 & 0.55 & 3.7 & 13.6 & 1,200 & 35.5 & 5.3 & & 4.5 & 2.1 & 304 & $<0.1$ & 2.9 & 0.4 & 1.0 & 41 & 0.5 & 6.5 & 103 & 0.6 & 7.51 \\
\hline & & & & unbl & 3720 & 0.60 & 4.8 & 12.1 & 1,260 & 48.7 & 5.6 & & 4.2 & 3.7 & 119 & $<0.1$ & 3.6 & 0.4 & 1.4 & 43 & 0.7 & 5.1 & 121 & 0.5 & 20.80 \\
\hline & & & & low $/ \mathrm{me}$ & 1170 & 0. & 5.2 & 7.9 & 870 & 36.8 & 5.2 & & 4.4 & 2.2 & 174 & $<0.1$ & 3.4 & 0.4 & 1.2 & 43 & 0.6 & 5.3 & & 0.4 & 12.40 \\
\hline & & & & hig & 924 & & 4.8 & 12.0 & 1,190 & 0.1 & & & 4.9 & 2.4 & 257 & $<0.1$ & 3.5 & 0.5 & 1.2 & 45 & & 5.6 & 109 & & 4.70 \\
\hline 7 & & & & unt & 103 & 60 & 4.0 & 7.1 & 560 & 21.4 & 1.2 & & 3.9 & 2.3 & 123 & $<0.1$ & 2.6 & 0.3 & 12.8 & 33 & 0.4 & 4.3 & 19 & 0.6 & 20.60 \\
\hline & & & & low $/ \mathrm{r}$ & 245 & 0. & 4.6 & 15.5 & 860 & 35.4 & 1.6 & & 4.6 & 2.7 & 106 & $<0.1$ & 5.3 & 0.3 & 5.1 & 46 & 1.8 & 5.7 & 53 & 0.6 & 20.50 \\
\hline & & & & high & 643 & 0.33 & 3.8 & 13.3 & 750 & 1.1 & & & 4.8 & 3.5 & 247 & $<0.1$ & 3.1 & 0. & 1.0 & 36 & 0.4 & 5.8 & 40 & 0.4 & 6.11 \\
\hline & & & & & 1150 & & 3.5 & 1.5 & 710 & & & & 5.0 & 2.0 & 195 & $<0.1$ & 3.0 & 0. & 1.0 & & 0.4 & 5. & 64 & 0.4 & 16.00 \\
\hline & & & & & 248 & & 7.4 & 17.9 & 660 & 3.9 & & & 6.6 & 2.1 & 162 & $<0.1$ & 4.6 & 0.4 & 5.1 & 70 & 0.7 & 6.2 & 47 & & 5.98 \\
\hline & & & & hig & & & 5 & 17.2 & 40 & & & & 5.6 & 2.3 & 190 & $<0.1$ & 4.6 & 0 & 2.1 & 60 & 0.5 & & 41 & & 8.21 \\
\hline & & & & unb & 570 & 0.58 & 3.3 & 6.1 & 780 & 46.7 & & & 3.4 & 2.3 & 181 & $<0.1$ & 2.7 & 0. & 0.8 & 24 & 0.4 & 4. & 76 & 0.5 & 20.10 \\
\hline & & & & & & & 3.0 & 6.4 & 70 & 3.4 & & & 3.2 & 2.6 & 180 & $<0.1$ & 2.4 & 0. & 0.8 & 23 & 0.4 & 4. & 75 & & 19.80 \\
\hline & & & & & 80 & 66 & 5.1 & 12.5 & 930 & 3.0 & & & 5.2 & 2.6 & 189 & $<0.1$ & 3.7 & 0.4 & 1.2 & 41 & 0.7 & 6.0 & 48 & 0.5 & 14.70 \\
\hline & & & & high & & & 5 & 16.4 & 1,580 & & & & 5.4 & 2.8 & 254 & $<0$. & 5. & 0.4 & 1.3 & 53 & 0.7 & 5.9 & 139 & 0.5 & 8.68 \\
\hline & & & & & & & & 16.8 & 1,030 & & & & 5.1 & & 182 & $<0$. & 3. & 0.4 & 1.1 & 46 & 0.7 & 6.0 & 131 & 6 & 12.40 \\
\hline & & & & low/medium & 1120 & 0.60 & 3.7 & 11.9 & 480 & 51.5 & & & 4.6 & 1.8 & 186 & $<0.1$ & 3.1 & 0.4 & 1.0 & 36 & 0.4 & 5.9 & 46 & & 15.60 \\
\hline & & & & high & 549 & 0.36 & 4.8 & 9.5 & 480 & 54.6 & 7.3 & & 4.8 & 1.4 & 228 & $<0.1$ & 3.5 & 0.5 & 1.2 & 37 & 0.4 & 6.6 & 68 & 0.5 & 5.40 \\
\hline & & & & high & 588 & & 4.8 & 10.7 & 520 & & & & 4.8 & & 233 & $<0.1$ & 3.5 & & 1.2 & & 0.5 & & & 0.6 & 6.14 \\
\hline & & & & & 412 & & & 7.7 & 560 & & & & 3.5 & 1.4 & 205 & $<0.1$ & 2.3 & & 0.8 & 26 & & & & & 16.30 \\
\hline SI & & & & & 342 & 0.28 & 2.8 & 9.0 & 470 & 26.6 & 0.9 & & 3.2 & 1.2 & 208 & $<0.1$ & 2.2 & 0.2 & 0.8 & 23 & 0.7 & 4.8 & 31 & 0.3 & 13.20 \\
\hline & & & & low/medium & 993 & 0.43 & 2.9 & 8.1 & 710 & 33.5 & 6.5 & & 3.2 & 1.9 & 188 & $<0.1$ & 2.6 & 0.3 & 0.8 & 23 & 0.3 & 4.0 & 48 & 0.4 & 17.30 \\
\hline & & & & high & & 0.48 & 4.0 & 12.7 & 740 & 3 & & & 4.2 & 0.9 & 218 & $<0.1$ & 3.1 & & 0.9 & 37 & 0.4 & 4. & 66 & .3 & 12.20 \\
\hline & & & & & & & & & 860 & & & & & & 173 & $<0.1$ & J.0 & & & 38 & & & & & 17.80 \\
\hline S & & 49617 & 2.88976 & low/medium & 1600 & 0.65 & 4.2 & 10.2 & 820 & 60.8 & 7.0 & & 4.3 & 2.1 & 198 & $<0.1$ & 3.4 & 0.3 & 1.0 & 36 & 1.0 & 5.3 & 79 & 0.4 & 17.00 \\
\hline & & 49626 & -92.88962 & high & 553 & 0.47 & 4.0 & 17.8 & 760 & 35.5 & 74.3 & 0.48 & 4.8 & 2.4 & 260 & $<0.1$ & 3.6 & 0.3 & 1.1 & 41 & 0.4 & 5.7 & 46 & 0.4 & 3.40 \\
\hline & & & & high & 619 & 0.28 & 2.6 & 9.8 & 620 & 19. & & & 3.4 & & 169 & $<0.1$ & 2.3 & & 0.8 & 25 & 0.3 & 3.9 & 37 & 0.4 & 17.00 \\
\hline & & & & & 468 & 0.70 & & 17.4 & 660 & & & & 4.4 & & 156 & $<0.1$ & 3.1 & & 7.0 & 45 & 0.5 & 5.8 & 62 & 0.7 & 16.30 \\
\hline SP-05-3-3A & 2005 & 48.49950| & -92.88430 & unburned & 378 & 0.51 & 4.0 & 14.6 & 440 & 27.3 & 55.0 & 0.28 & 4.1 & 2.6 & 173 & $<0.1$ & 3.1 & 0.2 & 3.7 & 35 & 0.4 & 4.8 & 34 & 0.4 & 16.60 \\
\hline
\end{tabular}




\begin{tabular}{|c|c|c|c|c|c|c|c|c|c|c|c|c|c|c|c|c|c|c|c|c|c|c|c|c|c|}
\hline Field no. & $\begin{array}{c}\text { Year } \\
\text { collected }\end{array}$ & atitude & ongitude & Fire severity & \begin{tabular}{|c|}
$\mathrm{Mn}$ \\
$(\mathrm{ppm})$
\end{tabular} & \begin{tabular}{|c|} 
Mo \\
(ppm)
\end{tabular} & \begin{tabular}{|c|}
$\begin{array}{c}\mathrm{Nb} \\
(\mathrm{ppm})\end{array}$ \\
\end{tabular} & $\begin{array}{c}\mathrm{Ni} \\
(\mathrm{ppm})\end{array}$ & \begin{tabular}{|c|}
$P$ \\
$(p p m)$
\end{tabular} & \begin{tabular}{|c|}
$\mathrm{Pb}$ \\
(ppm)
\end{tabular} & \begin{tabular}{|c|}
$\begin{array}{c}\mathrm{Rb} \\
(\mathrm{ppm})\end{array}$ \\
\end{tabular} & $\begin{array}{c}\mathrm{Sb} \\
(\mathrm{ppm})\end{array}$ & $\begin{array}{c}\mathrm{Sc} \\
(\mathrm{ppm})\end{array}$ & \begin{tabular}{|c|}
$\mathrm{Sn}$ \\
$(\mathrm{ppm})$
\end{tabular} & \begin{tabular}{|c}
$\mathrm{Sr}$ \\
(ppm)
\end{tabular} & $\begin{array}{c}\mathrm{Te} \\
(\mathrm{ppm})\end{array}$ & $\begin{array}{c}\text { Th } \\
(\mathrm{ppm})\end{array}$ & $\begin{array}{c}\mathrm{TI} \\
(\mathrm{ppm})\end{array}$ & \begin{tabular}{|c|}
$U$ \\
$(\mathrm{ppm})$ \\
\end{tabular} & $\begin{array}{c}V \\
(\mathrm{ppm})\end{array}$ & $\begin{array}{c}\mathrm{W} \\
(\mathrm{ppm})\end{array}$ & $\begin{array}{c}\mathrm{Y} \\
(\mathrm{ppm})\end{array}$ & \begin{tabular}{|c|}
$\mathrm{Zn}$ \\
$(\mathrm{ppm})$ \\
\end{tabular} & $\begin{array}{c}\text { Se } \\
(\mathrm{ppm})\end{array}$ & $\begin{array}{l}\text { Total C } \\
\text { (wt. \%) }\end{array}$ \\
\hline -05-4-1A & 2005 & 48.49866 & 2.89069 & unburned & 97 & \begin{tabular}{|l|}
0.49 \\
\end{tabular} & \begin{tabular}{|l|}
1.7 \\
\end{tabular} & 7.1 & \begin{tabular}{|l|}
830 \\
\end{tabular} & \begin{tabular}{|l|}
32.4 \\
\end{tabular} & \begin{tabular}{|l|}
16.8 \\
\end{tabular} & 0.48 & 1.7 & 1.6 & 57 & $<0.1$ & 1.6 & $<0.1$ & \begin{tabular}{|l|}
0.6 \\
\end{tabular} & 15 & 0.3 & 2.3 & 30 & 0.7 & 39.60 \\
\hline$-05-4-1 \mathrm{~A} d$ & & 49866 & 2.89069 & & 96 & 0.59 & 1.9 & 7.8 & 910 & 35.2 & 19.6 & 74 & 2.0 & 3.4 & 65 & $<0.1$ & 1.8 & 0.1 & 0.8 & 18 & 0.4 & 2.9 & 35 & 0.9 & 38.00 \\
\hline$-05-4-2 \mathrm{~A}$ & 05 & 9850 & 2.89060 & low/medium & 116 & 0.65 & 3.2 & 5.7 & 640 & 29.1 & 22.8 & 43 & 3.0 & 1.6 & 71 & $<0.1$ & 2.3 & 0.1 & 2.1 & 26 & 0.4 & 3.3 & 29 & 0.6 & 23.70 \\
\hline $05-4-3 \mathrm{~A}$ & & 19830 & & high & 607 & 0.50 & 4.1 & 16.4 & 850 & 189.0 & 74.1 & 96 & 4.9 & 2.6 & 247 & $<0.1$ & 3.7 & 0.3 & 1.1 & 41 & 0.4 & 5.8 & 84 & 0.6 & 6.69 \\
\hline $5-5-1 A$ & & 50055 & 88857 & unburned & 1140 & 0.27 & 2.7 & 11.6 & 750 & 29.9 & 58.1 & 0.26 & 3.7 & 2.0 & 193 & $<0.1$ & 2.7 & 0.2 & 0.9 & 28 & 0.3 & 4.2 & 64 & 0.4 & 15.60 \\
\hline & & & & low $/ \mathrm{m}$ & 667 & 0.27 & 2.8 & 9.5 & 520 & 4.0 & 69.1 & & 3.8 & 3.3 & 223 & $<0.1$ & 2.4 & 0.2 & 0.8 & 26 & 0.3 & 4.5 & 48 & 0.3 & 10.90 \\
\hline $5-3 \mathrm{~A}$ & & 50060 & 2.88840 & high & 672 & 0.51 & 3.5 & 11.2 & 650 & 41.5 & 64.2 & 0.56 & 4.1 & 1.6 & 231 & $<0.1$ & 2.9 & 0.3 & 0.9 & 31 & 0.4 & 5.2 & 70 & 0.4 & 13.00 \\
\hline $5-6-1 A$ & & 50142 & & unb & 2570 & 0.67 & 5.0 & 14.0 & 1,110 & 42.5 & 58.0 & 0.61 & 4.8 & 4.0 & 135 & $<0.1$ & 3.9 & 0.2 & 1.6 & 43 & 0.6 & 5.5 & 91 & 0.6 & 16.70 \\
\hline & & 70 & 10 & low $/ \mathrm{m}$ & 3050 & 0.53 & 3.2 & 10.4 & 1,390 & 40.2 & 38.6 & 0.54 & 3.1 & 1.9 & 135 & $<0.1$ & 2.7 & 0.2 & 1.0 & 28 & 0.5 & 3.9 & 108 & 0.7 & 24.40 \\
\hline & & & & high & 524 & 38 & 3.7 & 9.7 & 660 & 1.3 & 61.9 & & 4.0 & 4.4 & 211 & $<0.1$ & 3.0 & 0.3 & 1.0 & 31 & 0.4 & 5.0 & 68 & 0.4 & 4.07 \\
\hline & & & & & 63 & 0.37 & 1.5 & 6.4 & 810 & 24.1 & 21.4 & 19 & 1.7 & 1.9 & 71 & $<0.1$ & 1.5 & 0.1 & 7.1 & 15 & 0.3 & 2.3 & 35 & 0.5 & 38.20 \\
\hline & & & & & 596 & 0.75 & 3.1 & 13.5 & 1,140 & 51.4 & 7.2 & & 3.3 & 2.6 & 76 & $<0.1$ & 3.1 & 0.2 & 7.1 & 29 & 0.5 & 5.2 & 83 & 0.8 & 30.70 \\
\hline & & & & high & 844 & 0.37 & 4.1 & 14.7 & 2,060 & 0.5 & 82.7 & 57 & 4.7 & 2.3 & 239 & $<0.1$ & 3.6 & 0.4 & 1.1 & 39 & 0.5 & 6.1 & 69 & 0.5 & 8.41 \\
\hline & & & & & 1100 & 0.62 & 3.0 & 8.7 & 970 & 3.3 & 49.7 & & 3.2 & 1.8 & 142 & $<0.1$ & 2.5 & 0.3 & 0.9 & 31 & 0.4 & 3.8 & 69 & 0.5 & 22.90 \\
\hline & & & & & 160 & 0.67 & 5.4 & 15.9 & 1,150 & 37.3 & 9.5 & & 5.6 & 3.3 & 108 & $<0.1$ & 4.1 & 0.3 & 9.7 & 47 & 0.6 & 6.6 & 48 & 7 & 17.20 \\
\hline & & & & high & 264 & 0.90 & 4.8 & 16.2 & 860 & 32.0 & 50.4 & & 4.4 & 1.5 & 160 & $<0.1$ & 3.1 & 0.3 & 1.6 & 47 & 0.6 & 4.4 & 62 & 0.8 & 17.00 \\
\hline & & & & unb & 2380 & 0.48 & 3.0 & 10.9 & 900 & 37.0 & 67.5 & & 3.3 & 2.1 & 214 & $<0.1$ & 2.1 & 0.3 & 0.8 & 27 & 0.3 & 3.8 & 133 & 0.3 & 18.10 \\
\hline & & & & low $/ \mathrm{me}$ & 130 & 0. & 3.4 & 11.3 & $\mid 1,020$ & .4 & 58.0 & & 3.5 & 1. & 172 & $<0.1$ & 2.7 & 0.3 & 0.8 & 29 & 0.4 & 4.8 & 109| & & 25.80 \\
\hline & & & & hig & & 64 & 4.7 & .1 & 1,770 & 5.6 & 3.3 & & 4.4 & $4 .($ & 231 & $<0.1$ & 3.7 & 0 & 1.2 & 42 & & 5.2 & d & & 15.70 \\
\hline & & 10 & & unb & 3930 & 06 & 5.1 & 23.3 & 1,130 & 42.7 & 7.0 & & 5.5 & 4.5 & 216 & $<0.1$ & 3.6 & 0.5 & 1.3 & 56 & 0.8 & 6.1 & 142 & & 10.70 \\
\hline & & & & low $/ \mathrm{m}$ & 13 & 0. & 3.7 & 9.8 & 500 & 7.4 & .5 & & 4.3 & 2.0 & 173 & $<0.1$ & 3.6 & 0.3 & 1.0 & 33 & 0.4 & 5.1 & 48 & 0.6 & 16.10 \\
\hline & & & & high & 3 & 0.31 & 3. & 8.4 & 410 & 38.4 & 62.9 & & 4.0 & 4 & 202 & $<0.1$ & 2. & 0.4 & 1.1 & 31 & 0.4 & & 48 & & 9.78 \\
\hline & & & & high & 420 & & 4.0 & 11.7 & 460 & 0.9 & 3 & & 4.5 & & 212 & $<0.1$ & 2.9 & 0.3 & 1.1 & 32 & 0.4 & & 51 & & 9.88 \\
\hline & & & & unburned & 533 & & 3.3 & 5.2 & 520 & 7.5 & 7.2 & & 4.2 & 1.0 & 254 & $<0.1$ & 3.4 & 0.4 & 0.9 & 33 & 0.3 & 5. & 45 & 0. & 5.94 \\
\hline & & & & & & & 2 & 16.9 & 90 & & & & 4.0 & & 226 & $<0.1$ & 3.5 & 0.3 & 0.9 & 30 & 0.3 & 4.7 & 48 & 0.2 & 9.86 \\
\hline & & & & hig & 560 & 0.38 & 3.5 & 12.8 & 320 & .11 & 7.1 & & 3.6 & & 235 & $<0.1$ & 2.8 & 0.4 & 1.0 & 30 & 0.4 & 4.8 & 29 & $<0.2$ & 4.73 \\
\hline & & & & & & & 3.9 & 14.8 & 1,080 & & .4 & & 4.5 & 1.6 & 161 & $<0.1$ & 3.3 & 0.4 & 1.0 & 31 & 0.5 & 5. & 63 & 0.6 & 21.50 \\
\hline & & & & & 2890 & 70 & 4.1 & 14.8 & 900 & .7 & .0 & & 5.1 & 1.5 & 233 & $<0.1$ & 3.8 & 0.6 & 1.1 & 39 & 0.5 & 6.7 & 104 & 0.4 & 12.10 \\
\hline & & & & high & & & 4 & .4 & 30 & & & & 5.7 & & 274 & $<0$ & 3.8 & 0.4 & & 51 & 0.4 & & 31 & 0.4 & 1.81 \\
\hline & & & & hig & & & & & 590 & & & & 4.6 & & 235 & $<0.1$ & 4.4 & 0.4 & 1.0 & & 0.3 & & 41 & & 7.80 \\
\hline & & & & low/medium & 387 & 0.77 & 4.7 & 17.2 & 460 & 35.0 & 52.7 & & 4.7 & 1.3 & 174 & $<0.1$ & 3.3 & 0.3 & 5.4 & 45 & 0.5 & 5.8 & 51 & 0.5 & 14.30 \\
\hline & & & & & 708 & 0.77 & 4.1 & 14.2 & 480 & 32.1 & 4.0 & & 4.3 & 1.1 & 195 & $<0.1$ & 2.8 & 0.4 & 3.7 & 40 & 0.4 & 5.2 & 48 & 0.4 & 12.70 \\
\hline & & & & & & & & 15.0 & 420 & & & & 3.9 & & 179 & $<0.1$ & 3.3 & & 3.7 & & & & & 0.4 & \\
\hline & & & & & 2290 & & & 11.9 & 770 & & & & & & & $<0.1$ & & & & 45 & & & & & 14.60 \\
\hline & & & & low/medium & 260 & 0.91 & 4.3 & 7.5 & $\mid 1,060$ & 34.2 & 36.8 & & 4.5 & 1.8 & 107 & $<0.1$ & 3.4 & 0.3 & 6.7 & 27 & 0.6 & 5.4 & 42 & 0.6 & 22.30 \\
\hline & & & & high & 494 & 0.44 & 3.3 & 17.2 & 630 & 22.7 & 78.6 & & 5.3 & 1.0 & 244 & $<0.1$ & 5.0 & 0.4 & 1.0 & 38 & 0.3 & 5.2 & 53 & 0.5 & 4.82 \\
\hline & & & & & 1680 & & & 15.8 & 720 & & 72.4 & & 3.6 & & 193 & $<0.1$ & 3.3 & 0.4 & 0.9 & 29 & 0.4 & & 86 & 0.4 & 15.20 \\
\hline & & & & & 389 & & & & 220 & & & & & & 254 & $<0.1$ & 3.3 & & & 30 & 0.2 & & 23 & $<0.2$ & 3.30 \\
\hline & & 50060 & 2.88840 & high & 546 & 0.65 & 2.9 & 11.5 & 660 & 41.3 & 53.1 & & 4.1 & 1.3 & 182 & $<0.1$ & 2.4 & 0.4 & 0.8 & 31 & 0.3 & 4.7 & 50 & 0.6 & 22.30 \\
\hline 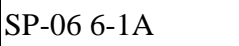 & & 50142 & -92.88990 & unburned & 1110 & 0.50 & 3.1 & 11.1 & 530 & 30.3 & 58.8 & & 4.6 & 1.1 & 202 & $<0.1$ & 2.7 & 0.4 & 0.9 & 30 & 2.1 & 5.1 & 46 & 0.3 & 11.40 \\
\hline & & & & low/medium & 2800 & 0.88 & 4.5 & 15.0 & $|1,200|$ & 50.5 & 70.8 & & 5.4 & & 187 & $<0.1$ & 3.8 & 0.5 & 1.3 & 43 & 0.6 & 6.4 & 120 & 0.7 & 14.20 \\
\hline & & & & high & & & & 8.7 & 750 & & 65.1 & & 4.9 & & 230 & $<0.1$ & 3.2 & & 1.1 & 34 & 0.4 & & 65 & 0.4 & 5.77 \\
\hline SP-06 6-3A dup & 2006 & 48.50180 & -92.89100 & high & 805 & 0.50 & 3.7 & 9.6 & 820 & 35.1 & 63.3 & 0.48 & 4.3 & 1.3 & 230 & $<0.1$ & 3.2 & 0.4 & 1.1 & 34 & 0.4 & 5.6 & 75 & 0.4 & 5.85 \\
\hline
\end{tabular}


Appendix 4-A Horizon Chemistry, Shoepack Lake, Voyageurs National Park, MN

\begin{tabular}{|c|c|c|c|c|c|c|c|c|c|c|c|c|c|c|c|c|c|c|c|c|c|c|c|c|c|}
\hline Field no. & $\begin{array}{c}\text { Year } \\
\text { collected }\end{array}$ & Latitude & Longitude & Fire severity & $\begin{array}{c}\mathrm{Mn} \\
(\mathrm{ppm})\end{array}$ & $\begin{array}{c}\text { Mo } \\
(\mathrm{ppm})\end{array}$ & $\begin{array}{c}\mathrm{Nb} \\
\text { (ppm) }\end{array}$ & $\begin{array}{c}\mathrm{Ni} \\
(\mathrm{ppm})\end{array}$ & $\begin{array}{c}\mathbf{P} \\
(\mathrm{ppm})\end{array}$ & $\begin{array}{c}\mathrm{Pb} \\
(\mathrm{ppm})\end{array}$ & $\begin{array}{c}\mathrm{Rb} \\
(\mathrm{ppm})\end{array}$ & $\begin{array}{c}\mathrm{Sb} \\
(\mathrm{ppm})\end{array}$ & $\begin{array}{c}\mathrm{Sc} \\
(\mathrm{ppm})\end{array}$ & $\begin{array}{c}\mathrm{Sn} \\
(\mathrm{ppm})\end{array}$ & $\begin{array}{c}\mathrm{Sr} \\
(\mathrm{ppm})\end{array}$ & $\begin{array}{c}\mathrm{Te} \\
(\mathrm{ppm})\end{array}$ & $\begin{array}{c}\text { Th } \\
\text { (ppm) }\end{array}$ & $\left.\begin{array}{c}\mathrm{Tl} \\
(\mathrm{ppm})\end{array}\right)$ & $\begin{array}{c}U \\
(\mathrm{ppm})\end{array}$ & $\begin{array}{c}\mathrm{V} \\
(\mathrm{ppm})\end{array}$ & $\begin{array}{c}\text { W } \\
\text { (ppm) }\end{array}$ & $\begin{array}{c}\mathrm{Y} \\
(\mathrm{ppm})\end{array}$ & $\begin{array}{c}\mathrm{Zn} \\
(\mathrm{ppm}) \\
\end{array}$ & \begin{tabular}{|c|}
$\mathrm{Se}$ \\
$(\mathrm{ppm})$ \\
\end{tabular} & $\begin{array}{l}\text { Total C } \\
\text { (wt. \%) }\end{array}$ \\
\hline SP-06 7-1A & 2006 & 48.50540 & -92.89810 & unburned & 99 & 0.80 & 2.6 & 9.4 & 1,170 & 22.4 & 32.3 & 0.41 & 3.8 & 0.9 & $\begin{array}{ll}77 \\
\end{array}$ & $<0.1$ & 2.3 & 0.2 & 24.9 & 26 & 0.3 & 4.7 & 26 & \begin{tabular}{|l|}
0.5 \\
\end{tabular} & 28.60 \\
\hline SP-06 7-2A & 2006 & 48.50501 & -92.89789 & low/medi & 527 & 0.90 & 3.3 & 16.8 & 1,100 & 33.0 & 45.2 & 0.75 & 5.0 & 1.3 & 108 & $<0.1$ & 3.4 & 0.4 & 6.1 & 36 & 0.5 & 6.2 & 61 & 0.8 & 24.20 \\
\hline SP-06 7-3A & $200 €$ & 48.50480 & -92.89560 & high & 1350 & 0.48 & 3.7 & 17.3 & 1,560 & 51.3 & 80.3 & 0.56 & 5.6 & 1.5 & 240 & $<0.1$ & 3.4 & 0.5 & 1.0 & 40 & 0.4 & 6.8 & 79 & 0.6 & 8.05 \\
\hline SP-06 8-1A & 2006 & 50166 & $\mid-92.8$ & unburned & 2770 & 0.65 & 3.9 & 13.3 & 470 & 27.7 & 61.1 & 0.36 & 5.0 & 1.1 & 226 & $<0.1$ & 3.6 & 0.4 & 1.0 & 37 & 0.4 & 5.8 & 51 & 0.3 & 5.52 \\
\hline SP-06 8-2A & 2006 & 50170 & 7230 & low/r & 227 & 1.05 & 5.0 & 21.1 & 1,420 & 39.8 & 53.5 & 0.51 & 6.8 & 1.5 & 125 & $<0.1$ & 4.1 & 0.4 & 10.5 & 53 & 0.5 & 7.2 & 49 & 0.6 & 16.90 \\
\hline P-06 8-2 & 2006 & 50170 & -92. & low $/ \mathrm{m}$ & 219 & 1.03 & 5.0 & 19.5 & 1,420 & 38.4 & 53.1 & 0.53 & 6.1 & 1.5 & 120 & $<0.1$ & 4.2 & 0.4 & 10.8 & 51 & 0.5 & 7.1 & 49 & 0.7 & 17.00 \\
\hline SP-06 8-3A & 2006 & 48.50180 & \begin{tabular}{|l|l} 
& -92.87250
\end{tabular} & high & 531 & 0.73 & 5.4 & 13.4 & 810 & 28.4 & 64.2 & 0.43 & 5.6 & 1.8 & 214 & $<0.1$ & 3.9 & 0.4 & 1.8 & 47 & 0.5 & 6.6 & 53 & 0.3 & 5.85 \\
\hline SP-06 9-1A & 2006 & 48.50080 & -92.87210 & unburned & 1980 & 0.69 & 2.8 & 9.5 & 880 & 43.8 & 56.0 & 0.46 & 3.9 & 1.3 & 195 & $<0.1$ & 2.7 & 0.3 & 0.8 & 26 & 0.3 & 4.5 & 99 & 0.4 & 18.10 \\
\hline SP-06 9-2A & 2006 & 48.50097 & \begin{tabular}{|l|l} 
& -92.87247
\end{tabular} & low/medium & 3230 & 0.75 & 3.2 & 14.6 & 960 & 40.9 & 54.7 & 0.58 & 4.5 & 1.2 & 172 & $<0.1$ & 2.9 & 0.4 & 0.9 & 33 & 0.4 & 5.2 & 83 & 0.7 & 20.60 \\
\hline P-06 9-3A & 2006 & 48.50130 & \begin{tabular}{|l|l} 
& -92.87270
\end{tabular} & high & 1660 & 0.63 & 4.6 & 14.0 & 760 & 44.9 & 63.5 & 0.44 & 5.3 & 1.5 & 209 & $<0.1$ & 3.5 & 0.4 & 1.1 & 41 & 0.4 & 6.4 & 113 & 0.4 & 10.10 \\
\hline P-06 10-1A & 200 & & & unburned & 3860 & 0.86 & 4.3 & 16.6 & 1,110 & 42.0 & 64.6 & 0.50 & 5.9 & 1.2 & 203 & $<0.1$ & 4.1 & 0.4 & 1.1 & 46 & 0.5 & 6.7 & 150 & 0.5 & 11.80 \\
\hline SP-06 10- & 2006 & 0192 & \begin{tabular}{|l|l} 
& -92.88459
\end{tabular} & low/medium & 2460 & 0.96 & 4.2 & 15.1 & 590 & 44.4 & 73.7 & 0.52 & 5.5 & 1.3 & 206 & $<0.1$ & 3.8 & 0.5 & 1.2 & 43 & 0.5 & 6.5 & 01 & 0.5 & 11.40 \\
\hline P-06 10-3A & 2006 & 48.50180 & \begin{tabular}{|l}
-92.88450 \\
\end{tabular} & high & 639 & 0.47 & 3.6 & 9.7 & 380 & 42.8 & 61.8 & 0.42 & 4.4 & 1.3 & 207 & $<0.1$ & 3.1 & 0.5 & 1.1 & 31 & 0.5 & 5.9 & 58 & 0.4 & 9.33 \\
\hline
\end{tabular}


Click back to Contents

Appendix 5-B Horizon Chemistry, Shoepack Lake, Voyageurs National Park, MN

\begin{tabular}{|c|c|c|c|c|c|c|c|c|c|c|c|c|c|c|c|c|c|c|c|c|c|c|c|c|c|c|c|c|}
\hline Field no. & $\begin{array}{c}\text { Year } \\
\text { collected }\end{array}$ & Latitude & Longitude & Fire severity & $\begin{array}{c}\mathrm{Al} \\
\text { (wt. \%) }\end{array}$ & \begin{tabular}{c|}
$\mathrm{Ca}$ \\
(wt. \%)
\end{tabular} & \begin{tabular}{|c|}
$\mathrm{Fe}$ \\
(wt. \%)
\end{tabular} & \begin{tabular}{|c|}
$\mathrm{K}$ \\
(wt. \%)
\end{tabular} & $\begin{array}{c}M g \\
\text { (wt. \%) }\end{array}$ & $\begin{array}{c}\mathrm{Na} \\
\text { (wt. \%) }\end{array}$ & \begin{tabular}{|c|}
$\mathbf{S}$ \\
(wt. \%)
\end{tabular} & \begin{tabular}{c|}
$\mathrm{Ti}$ \\
(wt. \%)
\end{tabular} & $\begin{array}{c}\mathrm{Ag} \\
(\mathrm{ppm})\end{array}$ & \begin{tabular}{|c|} 
As \\
$(\mathrm{ppm})$
\end{tabular} & $\begin{array}{c}\mathrm{Ba} \\
(\mathrm{ppm})\end{array}$ & \begin{tabular}{c|}
$\mathrm{Be}$ \\
$(\mathrm{ppm})$
\end{tabular} & \begin{tabular}{c|}
$\mathrm{Bi}$ \\
$(\mathrm{ppm})$
\end{tabular} & \begin{tabular}{c|}
$\mathrm{Cd}$ \\
$(\mathrm{ppm})$
\end{tabular} & \begin{tabular}{c|}
$\mathrm{Ce}$ \\
$(\mathrm{ppm})$
\end{tabular} & \begin{tabular}{|c|} 
Co \\
$(\mathrm{ppm})$
\end{tabular} & \begin{tabular}{c|}
$\mathrm{Cr}$ \\
$(\mathrm{ppm})$
\end{tabular} & $\begin{array}{c}\mathbf{C s}^{*} \\
(\mathrm{ppm})\end{array}$ & $\begin{array}{c}\mathrm{Cu} \\
(\mathrm{ppm})\end{array}$ & \begin{tabular}{c|}
$\mathrm{Ga}$ \\
$(\mathrm{ppm})$
\end{tabular} & \begin{tabular}{|c|}
$\mathrm{Hg}$ \\
$(\mathrm{ppm})$
\end{tabular} & \begin{tabular}{|c|} 
In \\
$(\mathrm{ppm})$
\end{tabular} & \begin{tabular}{c|}
$\mathrm{La}$ \\
$(\mathrm{ppm})$
\end{tabular} & $\begin{array}{c}\mathrm{Li} \\
(\mathrm{ppm})\end{array}$ \\
\hline SP-04-1-1B & 2004 & 48.49490 & -92.88660 & unburned & 6.13 & 1.20 & 1.84 & 1.54 & 0.47 & 2.10 & 0.01 & 0.19 & $<1$ & 2 & 425 & 1.4 & 0.15 & $<0.1$ & 15.6 & 8.7 & 47 & 2.24 & 6.9 & 13.4 & \begin{tabular}{|l|}
0.02 \\
\end{tabular} & 0.02 & 8.3 & 23 \\
\hline SP-04-1-2B & 2004 & 48.49495 & -92.88618 & low/medium & 6.33 & 1.24 & 1.69 & 1.57 & 0.42 & 2.17 & 0.01 & 0.17 & $<1$ & 2 & 421 & 1.3 & 0.17 & $<0.1$ & 16.1 & 6.1 & 42 & 2.30 & 7.7 & 13.1 & 0.02 & $<0.02$ & 8.4 & 20 \\
\hline SP-04-1-3B & 2004 & 49508 & -92.8858 & high & 6.27 & 1.23 & 1.85 & 1.49 & 0.42 & 2.01 & 0.02 & 0.21 & $<1$ & 4 & 421 & 1.4 & 0.26 & 0.1 & 17.2 & 6.4 & 47 & 3.21 & 34.8 & 14.1 & 0.03 & 0.03 & 9.2 & 19 \\
\hline SP-04-2-1B & 2004 & 49599 & -92.88988 & unburned & 6.22 & 1.31 & 68 & 1.80 & 0.56 & 2.02 & 0.02 & 0.22 & $<1$ & 3 & 525 & 1.2 & 0.13 & $<0.1$ & 20.3 & 7.8 & 48 & 2.14 & 8.9 & 13.4 & 0.05 & 0.03 & 10.6 & 13 \\
\hline SP-04-2-2B & 2004 & 48.49617 & -92.88976 & low/medium & 6.00 & 1.27 & 1.64 & 1.62 & 0.41 & 2.01 & 0.02 & 0.20 & $<1$ & 2 & 450 & 1.1 & 0.14 & $<0.1$ & 17.5 & 5.0 & 38 & 1.58 & 6.2 & 13.5 & 0.03 & 0.02 & 9.5 & 16 \\
\hline SP-04-2-3B & 2004 & 48.49626 & -92.88962 & high & 6.17 & 1.22 & 1.47 & 1.69 & 0.35 & 2.22 & 0.01 & 0.18 & $<1$ & 2 & 415 & 1.3 & 0.41 & $<0.1$ & 15.8 & 4.2 & 33 & 1.61 & 5.7 & 13.7 & $<0.02$ & $<0.02$ & 8.4 & 14 \\
\hline SP-04-3-1B & 2004 & 49990 & -92.88320 & high & 6.30 & 1.27 & 1.50 & 1.65 & 0.40 & 2.26 & 0.01 & 0.16 & $<1$ & 2 & 417 & 1.3 & 0.19 & $<0.1$ & 15.4 & 5.0 & 35 & 1.52 & 5.8 & 12.3 & 0.02 & $<0.02$ & 8.2 & 16 \\
\hline SP-04-3-2B & 2004 & 49950 & -92.88 & low/medium & 6.15 & 99 & 2.26 & 1.41 & 42 & 1.60 & 1 & 0.27 & $<1$ & 4 & 434 & 1.0 & 0.26 & $<0.1$ & 26.3 & 6.4 & 49 & 1.44 & 17.3 & 15.0 & 0.03 & 0.03 & 14.4 & 18 \\
\hline SP-04-4-1B & 2004 & 48.49866 & -92.89 & unburned & 5.69 & 0.83 & 1.96 & 1.49 & 0.45 & 1.36 & 0.02 & 0.25 & $<1$ & 3 & 458 & 1.0 & 0.21 & 0.1 & 22.1 & 6.8 & 46 & 1.04 & 13.6 & 14.5 & 0.05 & 0.03 & 12.7 & 18 \\
\hline SP-04-4-1B du & 2004 & 48.49866 & -92.890 & unburn & 5.61 & 0.85 & 1.89 & 1.43 & 0.45 & 1.35 & 0.03 & 0.24 & $<1$ & 3 & 452 & 1.0 & 0.23 & 0.1 & 22.4 & 6.3 & 44 & 2.39 & 15.5 & 14.2 & 0.04 & 0.03 & 12.5 & 17 \\
\hline SP-04-4-3B & 04 & 49830 & -92.89090 & high & 6.19 & 18 & 1.65 & .71[ & 41 & 2.02 & 0 & 0.18 & $<1$ & 3 & 423 & 1.1 & 0.14 & $<0.1$ & 15.7 & 5.4 & 37 & 2.79 & 7.8 & 13.2 & 0.03 & 0.02 & 8.3 & 16 \\
\hline SP-04-5-1B & 2004 & 48.50055 & -92.88857 & unburned & 6.17 & 1.28 & 1.42 & 1.83 & 0.38 & 2.24 & 0.01 & 0.16 & $<1$ & 2 & 474 & 1.2 & 0.11 & $<0.1$ & 13.6 & 4.4 & 29 & 3.24 & 5.4 & 12.8 & 0.03 & $<0.02$ & 7.0 & 14 \\
\hline SP-04-5-2B & 2004 & 48.50060 & $-92.8 \varepsilon$ & low/medium & 6.10 & 1.28 & 1.32 & 1.73 & 0.32 & 2.30 & 0.01 & 0.15 & $<1$ & 1 & 405 & 1.4 & 0.09 & $<0.1$ & 14.1 & 4.0 & 26 & 2.05 & 5.3 & 12.0 & $<0.02$ & $<0.02$ & 7.4 & 11 \\
\hline SP-04-5-3B & 2004 & 48.50060 & -92.88 & high & 6.04 & 1.31 & 1.33 & 1.58 & 0.35 & 2.22 & 0.02 & 0.17 & $<1$ & 2 & 400 & 1.3 & 0.09 & $<0.1$ & 13.7 & 4.2 & 31 & 3.74 & 4.6 & 12.5 & $<0.02$ & $<0.02$ & 7.3 & 12 \\
\hline SP-04-6-1B & 2004 & 48.50142 & -92.88990 & unburne & 6.18 & 0.97 & 2.62 & 1.26 & 0.46 & 1.4 & 0.03 & 0.24 & $<1$ & 4 & 424 & 1.7 & 0.47 & $<0.1$ & 21.8 & 6.8 & 54 & 5.67 & 24.2 & 14.6 & 0.07 & 0.03 & 11.8 & 28 \\
\hline SP-04-6-2B & 04 & 50170 & -92.8 & low $/ \mathrm{m}$ & 5.96 & & & 46 & 37 & 58 & & 23 & $<1$ & 4 & 420 & 1.1 & 0.35 & $<0.1$ & 17.7 & 5.5 & 42 & 2.44 & 10.5 & 16.2 & 0.05 & 0.03 & 9.8 & 23 \\
\hline SP-04-6-3B & 2004 & 48.50180 & & high & 5.61 & & 1. & 1.51 & 24 & 98 & & 18 & $<1$ & 2 & 418 & 1.2 & 0.13 & $<0.1$ & 12.7 & 2.6 & 26 & 1.34 & 5.9 & 12.6 & 0.02 & $<0.02$ & 6.8 & 8 \\
\hline SP-04-6-3B d & 2004 & 48.50180 & -92.89 & high & 5.51 & 1.11 & 1.09 & 1.48 & 0.25 & 1.96 & 0. & 0.18 & $<1$ & 2 & 400 & 1.1 & 0.15 & $<0.1$ & 15.2 & 2.8 & 26 & 1.72 & 6.0 & 12.5 & 0.02 & $<0.02$ & 8.1 & 8 \\
\hline SP-04-7-3B & 2004 & 48.50480 & 2.89560 & high & 6.15 & 1.27 & 1.23 & 1.72 & 31 & 2.38 & & & $<1$ & 2 & 407 & 1.4 & 0.15 & 0.1 & 12.4 & 4.1 & 28 & 1.60 & 5.0 & 12.1 & 0.02 & $<0.02$ & 6.4 & 11 \\
\hline SP-04-8-1B & 2004 & 48.50166 & -92.87 & unburn & 5.93 & 1.16 & 1.83 & 1.33 & & & & 0.20 & $<1$ & 3 & 476 & 1.3 & 0.26 & 0.2 & 16.9 & 7.2 & 46 & 1.85 & 11.3 & 13.1 & 0.05 & 0.03 & 8.7 & 18 \\
\hline SP-04-8-2B & 2004 & 48.50170 & -92.87230 & low/medium & 6.60 & 0.91 & 2.44 & 1.68 & 0.63 & & & 0.29 & $<1$ & 4 & 551 & 1.2 & 0.22 & $<0.1$ & 28.8 & 9.0 & 62 & 3.07 & 17.0 & 16.4 & 0.03 & 0.04 & 15.0 & 24 \\
\hline SP-04-8-2B dup & 2004 & 48.50170 & -92.87230 & low/medium & 6.74 & 0.94 & 2.45 & 1.73 & 63 & 1.50 & 0.02 & 0.29 & $<1$ & 4 & 565 & 1.0 & 0.22 & $<0.1$ & 27.6 & 9.5 & 69 & 3.68 & 18.1 & 16.1 & 0.03 & 0.03 & 14.3 & 25 \\
\hline SP-04-8-3B & 2004 & 48.50180 & -92.87250 & high & 6.69 & 1.06 & 2.41 & 35 & 51 & 1.78 & 0.03 & & $<1$ & 4 & 396 & 1.5 & 0.23 & $<0.1$ & 17.5 & 8.5 & 66 & 2.90 & 15.4 & 14.9 & 0.07 & 0.03 & 9.8 & 27 \\
\hline SP-04-9-1 & 2004 & 30 & -92.8 & unburn & 5.79 & & & & & & & & $<1$ & 2 & 472 & 1.1 & 0.13 & $<0.1$ & 16.7 & 4.1 & 28 & 1.59 & 3.9 & 11.8 & .02 & 0.02 & 9.7 & 11 \\
\hline SP-04-9-2B & 2004 & 48.50097 & -92.87247 & low/medium & 6.09 & 1.12 & 1.6 & 1.83 & 49 & 1. & & 0.26 & $<1$ & 3 & 550 & 1.2 & 0.17 & $<0.1$ & 25.1 & 7.4 & 47 & 2.00 & 6.8 & 13.8 & 0.03 & 0.03 & 13.9 & 16 \\
\hline SP-04-9-3B & 2004 & 48.50130 & -92.87270 & high & 6.87 & 1.21 & 2.57 & 1.28 & 0.63 & 1.95 & 0.02 & 0.22 & $<1$ & 4 & 369 & 1.4 & 0.28 & $<0.1$ & 17.3 & 8.1 & 63 & 2.76 & 13.1 & 14.7 & 0.04 & 0.03 & 9.8 & 31 \\
\hline SP-04-10-1B & 2004 & 48.50210 & -92.88490 & unburned & 6.42 & 1.30 & 2.37 & 1.41 & 51 & 1.85 & 02 & 0.24 & $<1$ & 3 & 686 & 1.3 & 0.21 & 0.3 & 21.3 & 9.7 & 53 & 3.71 & 12.6 & 15.1 & 0.06 & 0.03 & 11.0 & 24 \\
\hline & & & & low $/ \mathrm{m}$ & 6.50 & & & & & & & & $<1$ & 2 & 458 & 1.2 & 0.17 & $<0.1$ & 15.8 & 6.0 & 43 & 2.52 & 6.6 & 14.0 & 03 & 0.02 & 8.4 & 20 \\
\hline SP-04-10-3B & 2004 & 48.50180 & -92.88450 & high & 5.70 & & & & & & & & 1 & 2 & 448 & 1.2 & 0.11 & $<0.1$ & 15.8 & 3.9 & 31 & 1.72 & 8.2 & 12.5 & 0.02 & $<0.02$ & 8.5 & 11 \\
\hline SP-05-1-1B & 2005 & 48.49490 & -92.88660 & unburned & 5.06 & 1.05 & 0.71 & 1.51 & 0.21 & 2.03 & $<0.01$ & 0.11 & $<1$ & 1 & 417 & 1.4 & 0.06 & 0.2 & 14.7 & 2.0 & 10 & 0.81 & 1.6 & 11.5 & $<0.02$ & $<0.02$ & 8.0 & 5 \\
\hline SP-05-1-2B & 2005 & 48.49495 & -92.88618 & low/medium & 5.36 & 1.12 & 1.14 & 1.55 & 0.32 & 2.03 & 0.01 & 0.12 & $<1$ & 2 & 417 & 1.5 & 0.13 & $<0.1$ & 14.4 & 3.3 & 20 & 1.82 & 3.0 & 13.5 & $<0.02$ & $<0.02$ & 7.6 & 10 \\
\hline & 20 & & & high & & 1.09 & & & & & & & $<1$ & & 451 & 1.3 & 0.14 & $<0.1$ & 18.1 & 4.6 & 30 & 1.99 & 4.5 & 14.5 & 0.03 & 0.02 & 10.2 & 12 \\
\hline $05-2-1 B$ & 2005 & 49599 & -92.88988 & & & & & & & & & & $<1$ & 2 & & 1.3 & 0.12 & $<0.1$ & 23.0 & 7.6 & 34 & 1.26 & 4.9 & 14.8 & 03 & 0.03 & & 13 \\
\hline SP-05-2-2B & 2005 & 48.49617 & -92.88976 & low/medium & 5.37 & 1.20 & 1.05 & 1.53 & 31 & 2.07 & 0.01 & 0.14 & $<1$ & 1 & 432 & 1.3 & 0.10 & $<0.1$ & 25.3 & 2.9 & 16 & 0.78 & 3.8 & 13.3 & 0.03 & $<0.02$ & 13.0 & 7 \\
\hline SP-05-2-3B & 2005 & 48.49626 & -92.88962 & high & 5.80 & 1.18 & 1.73 & 1.54 & 0.41 & 2.11 & 0.01 & 0.15 & $<1$ & 2 & 396 & 1.3 & 0.15 & $<0.1$ & 16.0 & 4.6 & 28 & 1.30 & 3.6 & 14.8 & $<0.02$ & $<0.02$ & 8.6 & 16 \\
\hline$-05-2-3 B$ dup & 2005 & 48.49626 & -92.88962 & high & 6.37 & 1.30 & 1.7 & 1.6 & 0.43 & 2.2 & 0.01 & 0.16 & $<1$ & 4 & 409 & 1.4 & 0.17 & $<0.1$ & 15.0 & 4.2 & 24 & 2.95 & 3.5 & 15.8 & $<0.02$ & $<0.02$ & 8.1 & 18 \\
\hline$-05-3-1 \mathrm{~B}$ & 2005 & 48.49990 & -92.88320 & high & 5.53 & & 1.22 & & & & & & $<1$ & & 412 & 1.4 & 0.12 & $<0.1$ & 15.4 & 4.3 & 25 & 0.72 & 3.0 & 12.6 & 0.02 & $<0.02$ & 7.8 & 10 \\
\hline SP-05-3-2B & 2005 & 48.49950 & -92.88380 & low/medium & 5.55 & 0.88 & 2.23 & 1.16 & 0.39 & 1.48 & 0.02 & 0.20 & $<1$ & 3 & 399 & 1.3 & 0.15 & 0.1 & 21.3 & 4.6 & 28 & 1.93 & 11.5 & 14.6 & 0.05 & 0.02 & 11.7 & 18 \\
\hline
\end{tabular}


Appendix 5-B Horizon Chemistry, Shoepack Lake, Voyageurs National Park, MN

\begin{tabular}{|c|c|c|c|c|c|c|c|c|c|c|c|c|c|c|c|c|c|c|c|c|c|c|c|c|c|c|c|c|}
\hline Field no. & $\begin{array}{c}\text { Year } \\
\text { collected }\end{array}$ & Latitude & Longitude & Fire severity & $\begin{array}{c}\text { Al } \\
\text { (wt. \%) }\end{array}$ & $\begin{array}{c}\mathrm{Ca} \\
\text { (wt. \%) }\end{array}$ & $\begin{array}{c}\mathrm{Fe} \\
\text { (wt. \%) }\end{array}$ & $\begin{array}{c}\mathrm{K} \\
\text { (wt. \%) }\end{array}$ & $\begin{array}{c}\mathrm{Mg} \\
\text { (wt. \%) }\end{array}$ & $\begin{array}{c}\mathrm{Na} \\
\text { (wt. \%) }\end{array}$ & $\begin{array}{c}\mathrm{S} \\
\text { (wt. \%) }\end{array}$ & \begin{tabular}{|c}
$\mathrm{Ti}$ \\
(wt. \%)
\end{tabular} & $\begin{array}{c}\mathrm{Ag} \\
(\mathrm{ppm})\end{array}$ & $\begin{array}{c}\text { As } \\
\text { (ppm) }\end{array}$ & $\begin{array}{c}\mathrm{Ba} \\
(\mathrm{ppm})\end{array}$ & $\begin{array}{c}\mathrm{Be} \\
(\mathrm{ppm})\end{array}$ & $\begin{array}{c}\mathrm{Bi} \\
(\mathrm{ppm})\end{array}$ & $\begin{array}{c}\mathrm{Cd} \\
(\mathrm{ppm})\end{array}$ & $\begin{array}{c}\mathrm{Ce} \\
(\mathrm{ppm})\end{array}$ & $\begin{array}{c}\text { Co } \\
(\mathrm{ppm})\end{array}$ & $\begin{array}{c}\mathrm{Cr} \\
(\mathrm{ppm})\end{array}$ & $\begin{array}{c}\mathrm{Cs}^{*} \\
(\mathrm{ppm})\end{array}$ & $\begin{array}{c}\mathrm{Cu} \\
(\mathrm{ppm})\end{array}$ & $\begin{array}{c}\mathrm{Ga} \\
(\mathrm{ppm})\end{array}$ & $\begin{array}{c}\mathrm{Hg} \\
(\mathrm{ppm})\end{array}$ & \begin{tabular}{|c|} 
In \\
(ppm)
\end{tabular} & $\begin{array}{c}\mathrm{La} \\
(\mathrm{ppm})\end{array}$ & $\begin{array}{c}\mathrm{Li} \\
(\mathrm{ppm})\end{array}$ \\
\hline $\begin{array}{l}\text {-05-4-1B dup } \\
\end{array}$ & 2005 & 48.49866 & -92.89069 & unburned & 5.33 & $\begin{array}{l}0.77 \\
\end{array}$ & 1.86 & 1.27 & 0.37 & 1.26 & 0.02 & 0.21 & $\begin{array}{l}<1 \\
\end{array}$ & 3 & 433 & 1.2 & 0.22 & 0.1 & 24.7 & 5.0 & 22 & \begin{tabular}{|l|}
1.67 \\
\end{tabular} & 15.7 & 15.3 & $\begin{array}{l}0.04 \\
\end{array}$ & \begin{tabular}{|l|}
0.03 \\
\end{tabular} & \begin{tabular}{|l|}
13.5 \\
\end{tabular} & 15 \\
\hline P-05-4-2B & 55 & 49850 & 2.89060 & low/medium & 5.52 & 0.76 & 58 & 23 & 44 & 1.23 & 0.02 & 21 & $<1$ & 4 & 378 & 1.4 & 0.66 & 0.1 & 23.6 & 3.8 & 17 & 3.05 & 14.5 & 18.1 & 0.05 & 0.03 & 12.8 & \\
\hline$-05-4-3 B$ & 2005 & 49830 & 2.890 & high & 47 & 10 & & & & & 0.01 & & $<1$ & 2 & 438 & 1.3 & 0.12 & $<0.1$ & 14.7 & 4.4 & 19 & 1.13 & 4.6 & 13.5 & $<0.02$ & $<0.02$ & 790 & 11 \\
\hline P-05-5-1B & 2005 & 50055 & & unbun & .71 & 0.75 & & 89 & 23 & 1.22 & $<0.01$ & & $<1$ & 2 & 284 & 0.7 & 0.10 & 0.1 & 9.2 & 2.6 & 6 & 0.32 & 3.0 & 8.4 & 0.03 & $<0.02$ & 4.9 & \\
\hline SP-05-5-2B & 2005 & 50060 & 60 & lon & 54 & 1.16 & & & & 16 & .01 & 11 & $<1$ & 1 & 406 & 1.3 & 0.12 & $<0.1$ & 12.1 & 4.4 & 17 & 0.78 & 3.3 & 12.4 & $<0.02$ & $<0.02$ & 5.3 & \\
\hline P-05-5-3 & 2005 & 48.500 & 40 & high & 5.26 & 1.26 & & & 29 & 2.12 & $<0.01$ & 11 & $<1$ & $<1$ & 411 & 1.3 & 0.08 & $<0.1$ & 12.7 & 2.6 & 8 & 0.27 & 1.8 & 12.2 & $<0.02$ & $<0.02$ & 6.6 & \\
\hline-05 & & 42 & 0 & unbs & & & & & & & 0.02 & & $<1$ & 3 & 435 & 1.5 & 0.35 & 0.1 & 20.8 & 5.6 & 29 & 3.34 & 12.7 & 15.3 & 0.07 & \begin{tabular}{|l|} 
\\
\end{tabular} & 11.0 & \\
\hline P-05-6-2B & 2005 & & & low $/ \mathrm{m}$ & 92 & .93 & & & 32 & 36 & 0.02 & & $<1$ & 3 & 423 & 1.1 & 0.32 & 0.1 & 20.6 & 3.3 & 15 & 1.14 & 7.8 & 14.5 & 0.05 & 0.03 & 11.0 & 12 \\
\hline P-05- & 2005 & 50180 & - & high & 54 & 1.10 & & & & 02 & $<0.01$ & 15 & $<1$ & 2 & 402 & 1.2 & 0.13 & $<0.1$ & 14.0 & 3.4 & 7 & 1.50 & 3.3 & 14.1 & $<0.02$ & $<0.02$ & 8.1 & \\
\hline P-05 & 2005 & 48.50 & 0 & unburr & 53 & 0.98 & & & & 87 & 0.01 & 17 & $<1$ & 2 & 409 & 1.1 & 0.19 & $<0.1$ & 16.0 & 4.5 & 8 & 2.09 & 4.2 & 14.8 & 0.03 & 0.02 & 9.0 & \\
\hline-05 & 2005 & 01 & 9 & low $/ \mathrm{m}$ & & & & & & & 0.04 & & $<1$ & 5 & 435 & 2.5 & 0.38 & 0.1 & 28.1 & 25.9 & 53 & 5.89 & 34.1 & 15.2 & 0.09 & 0.04 & 15.2 & \\
\hline-05 & 2005 & & & high & & 29 & & & & 42 & $<0.01$ & & $<1$ & 2 & 407 & 1.5 & 0.13 & $<0.1$ & 14.2 & 7.0 & 30 & 1.19 & 5.2 & 13.4 & $<0.02$ & $<0.02$ & & 1 \\
\hline $\mathrm{P}-05$ & 05 & & & & & & & & & 36 & 0.02 & 18 & $<1$ & 3 & 431 & 1.4 & 0.21 & 0.1 & 15.8 & 6.0 & 36 & 1.11 & 5.6 & 14.3 & 0.04 & 0.03 & 8.5 & \\
\hline P-05 & 2005 & & -92 & low/ & 54 & 77 & & & & & 0.02 & 0 & $<1$ & 4 & 550 & 1.3 & 0.27 & $<0.1$ & 27.6 & 8.4 & 57 & 2.88 & 20.9 & 18.8 & 0.03 & 0.04 & 15.4 & \\
\hline P-05 & 20 & & 50 & hig & & & & & & & 0 & & $<1$ & 4 & 386 & 1.5 & 0.23 & 0.1 & $1 /$. & 6.1 & 50 & 2.11 & 11.8 & 15.8 & 0.07 & 0.03 & 9.7 & \\
\hline $\mathrm{P}-05$ & 2 & & & & & & & & & & $<0.01$ & & $<1$ & 2 & 440 & 1.3 & 0.15 & $<0.1$ & & 4.5 & 13 & 1.90 & 2.7 & 13.6 & $<0.02$ & $<0.02$ & 7.2 & \\
\hline SP-05 & & & & & & & & & & & 01 & & $<1$ & 2 & 431 & 1.3 & 0.12 & $<0.1$ & & 4.1 & 14 & & 3.2 & 12.9 & $<0.02$ & $<0.02$ & 7.9 & \\
\hline-05 & & & & low & & & & & 53 & & 0.01 & & $<1$ & 2 & 565 & 1.2 & 0.18 & $<0.1$ & 20.3 & 5.5 & 28 & 2.00 & 5.0 & 15.1 & 0.02 & 0.03 & 10.9 & \\
\hline P-05 & 20 & & & hig & & & & & & 38 & $<0.01$ & & $<1$ & 2 & 454 & 1.3 & 0.2 & 0. & 6 & 3.3 & 21 & 1.96 & 4.4 & 14.1 & 0.02 & 0.02 & 9.0 & \\
\hline P-05 & & & & & & & & & & & & & $<1$ & 3 & 565 & 1.4 & 0.2 & 0.2 & & 8.1 & 43 & 3.39 & 8.4 & 16.9 & 0.04 & 0.0 & 10.9 & \\
\hline -05 & & & & & & & & & & & & & $<1$ & 2 & 452 & 1.4 & 0.14 & $<0.1$ & & 4.7 & 25 & 2.47 & 4.0 & 4.1 & 0.02 & 0.0 & 8.2 & \\
\hline-05 & & & & high & & & & & & & & & $<1$ & $<1$ & 451 & 1.3 & 0.11 & $<0.1$ & & 1.9 & 9 & 2.07 & 2.0 & 13.0 & $<0.02$ & $<0.0$ & 7.7 & \\
\hline P-06 & 20 & 48 & -92.8 & unbu & & & & & & & $<0.01$ & & $<1$ & 1 & 413 & 1.3 & 0.02 & $<0$. & 17 & 4.6 & 21 & $<5$ & 11.5 & 13.1 & $<0.02$ & $<0.02$ & 9.0 & \\
\hline P-06 & & & & & & & & & & & & & $<1$ & 3 & 390 & 1.4 & 0.0 & $<0.1$ & & 6.8 & 32 & $<5$ & 7.6 & 13.3 & $<0.02$ & $<0.02$ & 8.3 & \\
\hline-06 & & & & high & & & & & & & & & $<1$ & 3 & 388 & 1.3 & 0.11 & $<0.1$ & & 7.5 & 46 & $<5$ & 7.6 & 4.5 & 0.02 & 0.02 & 11.3 & \\
\hline-06 & & & & & & & & & & & & & $<1$ & 2 & 537 & 1.4 & $0 .($ & $<0$. & 25.2 & 12.2 & 38 & $<5$ & 14.3 & 13.7 & & 0.0 & 13.3 & \\
\hline-06 & & & & low/ & & & & & & & & & $<1$ & 2 & 452 & 1.5 & 0.0 & $<0$. & & 6.7 & 30 & $<5$ & & 14.4 & $<0.02$ & 0.0 & & \\
\hline P-06 & & & & high & & & & & & & & & $<1$ & 4 & 388 & 1.5 & $0.0 €$ & $<0.1$ & & 9.7 & 39 & $<5$ & 5.9 & 14.0 & 0.02 & 0.0 & 8.2 & \\
\hline P-06 & & & & high & & & & & & & $<0.01$ & & $<1$ & 2 & 401 & 1.4 & 0.02 & $<0.1$ & & 8.1 & 27 & $<5$ & 5.1 & 13.4 & $<0.02$ & $<0.02$ & 9.0 & \\
\hline-06 & & & & & & & & & & & & & $<1$ & 4 & 382 & 1.1 & & 0 & & 7.1 & 33 & $<5$ & 12.4 & 15.4 & 0.0 & 0.02 & 12.2 & \\
\hline-06 & & & & & & & & & & & & & $<1$ & 3 & & & & $<0$. & & & & $<5$ & & 15.7 & $<0.02$ & 0.0 & & \\
\hline P-06 & & & & & & & & & & & & & $<1$ & 3 & 459 & 1.3 & & $<0.1$ & & 25.4 & 36 & $<5$ & 12.2 & 14.0 & 0.03 & 0.02 & 12.9 & \\
\hline P-06 4 & & & & & & & & & & & & & $<1$ & 4 & 448 & 1.4 & 0.07 & $<0.1$ & 20.0 & 23.0 & 50 & $<5$ & 7.8 & 14.0 & 0.03 & 0.02 & 10.9 & \\
\hline -06 & & & & & & & & & & & & & $<1$ & 4 & 274 & 1.7 & 0.3 & 0.2 & 22 & 3.4 & 35 & 6 & 12.0 & 15.3 & 0.05 & 0.03 & 13.5 & \\
\hline-06 & & & & hig & & & & & & & & & $<1$ & & & 1.4 & & $<0.1$ & & & & $<5$ & & 3.1 & $<0.02$ & $<0.02$ & & \\
\hline-06 & & & & & & & & & & & & & $<1$ & 2 & & & & & & & & $<5$ & & 11.9 & & $<0.02$ & & \\
\hline -06 & & 50060 & -92.88860 & low/medium & & & & & & 21 & $<0.01$ & & $<1$ & $<1$ & 407 & 1.2 & 0.02 & $<0.1$ & 11.0 & 3.9 & 29 & $<5$ & 3.1 & 12.2 & $<0.02$ & $<0.02$ & 6.0 & \\
\hline-06 & & & & |high & & & & & & & $<0.01$ & & $<1$ & $<1$ & 391 & 1.2 & 0.0 & $<0.1$ & & 5.9 & 38 & $<5$ & 2.7 & 13.0 & $<0.02$ & $<0.02$ & 7.2 & \\
\hline-06 & & & & & & & & & & & & & $<1$ & 2 & & 1.4 & & $<0.1$ & & & 41 & $<5$ & & 4.1 & .02 & 0.02 & 1.01 & 15 \\
\hline 00 & & & & & & & & & & & & & $<1$ & & & 1.6 & & $<0.1$ & & 10.7 & 40 & $<5$ & & 5.0 & & 0.04 & 10.2 & \\
\hline-06 6-2B dt & 20 & 50170 & -92.89010 & low/medium & 5.86 & 1.02 & 2.00 & & & 1.73 & 0.02 & & $<1$ & 4 & 387 & 1.1 & 0.20 & $<0.1$ & 16.2 & 5.9 & 43 & $<5$ & 8.6 & 13.8 & 0.04 & 0.02 & 8.8 & \\
\hline & & & & high & 80 & 1.15 & 1.53 & $1.0 \%$ & & 2.09 & .02 & & $<1$ & 3 & 391 & 0.9 & 0.21 & $<0.1$ & 16. & 4.6 & 38 & $<5$ & 6.6 & 14.6 & 0.02 & 0.02 & 8.7 & \\
\hline P-06 7-1B & 2006 & 48.50540 & -92.89810 & unburned & 6.23 & 0.95 & 1.99 & 1.77 & 0.49 & 1.68 & 0.02 & 0.23 & $<1$ & & 434 & 1.1 & 0.15 & $<0.1$ & 18.5 & 9.2 & 35 & $<5$ & 7.9 & 15.3 & 0.03 & 0.03 & 10.2 & \\
\hline
\end{tabular}


Appendix 5-B Horizon Chemistry, Shoepack Lake, Voyageurs National Park, MN

33

\begin{tabular}{|c|c|c|c|c|c|c|c|c|c|c|c|c|c|c|c|c|c|c|c|c|c|c|c|c|c|c|c|c|}
\hline Field no. & $\begin{array}{c}\text { Year } \\
\text { collected }\end{array}$ & Latitude & Longitude & Fire severity & $\begin{array}{c}\mathrm{Al} \\
\text { (wt. \%) }\end{array}$ & $\begin{array}{c}\mathrm{Ca} \\
\text { (wt. \%) }\end{array}$ & \begin{tabular}{c|}
$\mathrm{Fe}$ \\
(wt. \%)
\end{tabular} & $\begin{array}{c}\mathrm{K} \\
\text { (wt. \%) }\end{array}$ & $\begin{array}{c}\mathrm{Mg} \\
\text { (wt. \%) }\end{array}$ & $\begin{array}{c}\mathrm{Na} \\
\text { (wt. \%) }\end{array}$ & $\begin{array}{c}\mathbf{S} \\
\text { (wt. \%) }\end{array}$ & $\begin{array}{c}\mathrm{Ti} \\
\text { (wt. \%) }\end{array}$ & $\begin{array}{c}\mathrm{Ag} \\
(\mathrm{ppm})\end{array}$ & $\begin{array}{c}\text { As } \\
\text { (ppm) }\end{array}$ & \begin{tabular}{c|}
$\mathrm{Ba}$ \\
$(\mathrm{ppm})$
\end{tabular} & $\begin{array}{c}\mathrm{Be} \\
(\mathrm{ppm})\end{array}$ & \begin{tabular}{|c|}
$\mathrm{Bi}$ \\
$(\mathrm{ppm})$
\end{tabular} & $\begin{array}{c}\mathrm{Cd} \\
(\mathrm{ppm})\end{array}$ & $\begin{array}{c}\mathrm{Ce} \\
(\mathrm{ppm})\end{array}$ & $\begin{array}{c}\text { Co } \\
(\mathrm{ppm})\end{array}$ & $\begin{array}{c}\mathrm{Cr} \\
(\mathrm{ppm})\end{array}$ & $\begin{array}{c}\mathrm{Cs}^{*} \\
(\mathrm{ppm})\end{array}$ & $\begin{array}{c}\mathrm{Cu} \\
(\mathrm{ppm})\end{array}$ & $\begin{array}{c}\mathrm{Ga} \\
(\mathrm{ppm})\end{array}$ & $\begin{array}{c}\mathrm{Hg} \\
(\mathrm{ppm})\end{array}$ & \begin{tabular}{|c|} 
In \\
$(\mathrm{ppm})$
\end{tabular} & $\begin{array}{c}\text { La } \\
\text { (ppm) }\end{array}$ & $\begin{array}{c}\mathrm{Li} \\
\text { (ppm) }\end{array}$ \\
\hline$-067-2 B$ & 2006 & 48.50501 & -92.89789 & low/medium & 6.70 & 0.90 & \begin{tabular}{|l|}
3.27 \\
\end{tabular} & 1.28 & 0.56 & 1.52 & 0.02 & 0.22 & $<1$ & 4 & 380 & 1.9 & \begin{tabular}{|l|}
0.25 \\
\end{tabular} & $<0.1$ & \begin{tabular}{|l|}
22.3 \\
\end{tabular} & \begin{tabular}{r|}
19.3 \\
\end{tabular} & 61 & 6 & 18.6 & 15.0 & \begin{tabular}{l|}
0.05 \\
\end{tabular} & $\begin{array}{l}0.04 \\
\end{array}$ & 12.3 & 45 \\
\hline P-06 7-3B & 2006 & 48.50480 & -92.89560 & high & 6.30 & 1.31 & 1.22 & 1.85 & 0.34 & 2.35 & $<0.01$ & 0.14 & $<1$ & 2 & 407 & 1.6 & 0.05 & $<0.1$ & 13.6 & 7.1 & 32 & $<5$ & 5.3 & 12.7 & $<0.02$ & $<0.02$ & 7.4 & 13 \\
\hline P-06 8-1B & 2006 & 48.50166 & -92.87214 & unburned & 6.37 & 1.11 & 1.94 & 1.60 & 0.44 & 1.93 & 0.01 & 0.21 & $<1$ & 3 & 436 & 1.3 & 0.12 & 0.2 & 17.5 & 7.1 & 47 & $<5$ & 5.7 & 13.8 & 0.03 & 0.03 & 9.7 & 20 \\
\hline P-06 8-2B & 2006 & 48.50170 & -92.87230 & low/medium & 6.02 & 0.95 & 2.24 & 1.77 & 0.60 & 1.43 & 0.01 & 0.28 & $<1$ & 3 & 522 & 1.1 & 0.12 & $<0.1$ & 27.2 & 9.4 & 58 & $<5$ & 10.8 & 13.7 & $<0.02$ & 0.03 & 15.1 & 23 \\
\hline P-06 8-3B & 2006 & 48.50180 & -92.87250 & high & 6.24 & 0.96 & 2.30 & 1.50 & 0.47 & 1.60 & 0.02 & 0.22 & $<1$ & 4 & 415 & 1.3 & 0.25 & $<0.1$ & 25.5 & 5.1 & 46 & $<5$ & 10.7 & 17.0 & 0.03 & 0.03 & 13.7 & 21 \\
\hline P-06 8-3B dup & 2006 & 48.50180 & -92.87250 & high & 6.16 & 0.94 & 2.37 & 1.37 & 0.48 & 1.54 & 0.02 & 0.25 & $<1$ & 3 & 410 & 1.2 & 0.22 & 0.1 & 24.6 & 5.0 & 46 & $<5$ & 8.1 & 15.7 & 0.03 & 0.03 & 13.6 & 22 \\
\hline P-06 9-1B & 2006 & 48.50080 & -92.87210 & unburned & 5.65 & 1.13 & 1.01 & 1.80 & 0.31 & 1.99 & $<0.01$ & 0.17 & $<1$ & 2 & 469 & 1.3 & 0.02 & $<0.1$ & 18.7 & 3.6 & 29 & $<5$ & 2.5 & 12.5 & $<0.02$ & $<0.02$ & 10.2 & 9 \\
\hline P-06 9-2B & 2006 & 48.50097 & -92.87247 & low/medium & 6.48 & 1.20 & 1.80 & 1.93 & 0.55 & 1.95 & 0.01 & 0.21 & $<1$ & 3 & 523 & 1.5 & 0.07 & $<0.1$ & 20.0 & 7.6 & 54 & $<5$ & 9.8 & 14.8 & $<0.02$ & 0.03 & 11.1 & 15 \\
\hline P-06 9-3B & 2006 & 48.50130 & -92.87270 & high & 6.02 & 1.05 & 1.84 & 1.50 & 0.41 & 1.76 & 0.01 & 0.21 & $<1$ & 3 & 400 & 1.6 & 0.21 & $<0.1$ & 19.6 & 4.7 & 38 & $<5$ & 5.6 & 15.6 & 0.02 & 0.02 & 10.7 & 19 \\
\hline P-06 10-1B & 2006 & 48.50210 & -92.88490 & unburned & 6.46 & 1.21 & 2.57 & 1.58 & 0.62 & 1.79 & 0.01 & 0.23 & $<1$ & 3 & 488 & 1.4 & 0.12 & 0.1 & 17.5 & 8.6 & 58 & $<5$ & 8.4 & 15.2 & 0.03 & 0.03 & 9.9 & 27 \\
\hline P-06 10-2B & 2006 & 48.50192 & -92.88459 & low/medium & 6.28 & 1.23 & 1.75 & 1.74 & 0.48 & 2.01 & 0.01 & 0.19 & $<1$ & 5 & 487 & 1.1 & 0.08 & 0.1 & 18.2 & 8.2 & 44 & $<5$ & 4.8 & 13.6 & 0.04 & 0.03 & 9.6 & 20 \\
\hline P-06 10-3B & 2006 & 48.50180 & -92.88450 & high & 5.77 & 1.22 & 1.27 & 1.78 & 0.37 & 2.16 & $<0.01$ & 0.18 & $<1$ & 3 & 439 & 1.4 & 0.02 & $<0.1$ & 14.9 & 4.0 & 36 & $<5$ & 2.7 & 13.7 & $<0.02$ & $<0.02$ & 8.2 & 12 \\
\hline
\end{tabular}

*Because of analytical laboratory issues, Cs detection limits in 2004 and 2005 were $0.05 \mathrm{ppm}$ and 5 ppm in 2006. 
Appendix 5-B Horizon Chemistry, Shoepack Lake, Voyageurs National Park, MN

\begin{tabular}{|c|c|c|c|c|c|c|c|c|c|c|c|c|c|c|c|c|c|c|c|c|c|c|c|c|c|}
\hline Field no. & $\begin{array}{c}\text { Year } \\
\text { collected }\end{array}$ & Latitude & Longitude & Fire severity & \begin{tabular}{|c|}
$\mathrm{Mn}$ \\
$(\mathrm{ppm})$ \\
\end{tabular} & \begin{tabular}{|c|} 
Mo \\
(ppm)
\end{tabular} & \begin{tabular}{|c|}
$\begin{array}{c}\mathrm{Nb} \\
(\mathrm{ppm})\end{array}$ \\
\end{tabular} & \begin{tabular}{|c|}
$\mathrm{Ni}$ \\
$(\mathrm{ppm})$
\end{tabular} & \begin{tabular}{|c} 
P \\
(ppm)
\end{tabular} & \begin{tabular}{|c|}
$\begin{array}{c}\mathrm{Pb} \\
(\mathrm{ppm})\end{array}$ \\
\end{tabular} & \begin{tabular}{|c|}
$\mathrm{Rb}$ \\
$(\mathrm{ppm})$
\end{tabular} & $\begin{array}{c}\mathrm{Sb} \\
(\mathrm{ppm})\end{array}$ & $\begin{array}{c}\mathrm{Sc} \\
(\mathrm{ppm})\end{array}$ & $\begin{array}{c}\mathrm{Sn} \\
(\mathrm{ppm})\end{array}$ & \begin{tabular}{|c}
$\mathrm{Sr}$ \\
(ppm)
\end{tabular} & \begin{tabular}{|c|}
$\begin{array}{c}\mathrm{Te} \\
(\mathrm{ppm})\end{array}$ \\
\end{tabular} & \begin{tabular}{|c|} 
Th \\
$(\mathrm{ppm})$
\end{tabular} & \begin{tabular}{|c|}
$\mathrm{TI}$ \\
$(\mathrm{ppm})$
\end{tabular} & \begin{tabular}{|c|}
$U$ \\
$(\mathrm{ppm})$ \\
\end{tabular} & \begin{tabular}{|c|}
$\mathrm{V}$ \\
$(\mathrm{ppm})$
\end{tabular} & \begin{tabular}{|c|} 
W \\
$(\mathrm{ppm})$
\end{tabular} & \begin{tabular}{|c|}
$Y$ \\
$(\mathrm{ppm})$
\end{tabular} & \begin{tabular}{|c|}
$\mathrm{Zn}$ \\
(ppm)
\end{tabular} & \begin{tabular}{|c|}
$\begin{array}{c}\text { Se } \\
(\mathrm{ppm})\end{array}$ \\
\end{tabular} & $\begin{array}{l}\text { Total C } \\
\text { (wt. \%) }\end{array}$ \\
\hline SP-04-1-1B & 2004 & 48.49490 & -92.88660 & unburned & 312 & $\begin{array}{l}0.55 \\
\end{array}$ & \begin{tabular}{|l|}
5.5 \\
\end{tabular} & 23.1 & 410 & \begin{tabular}{|l|}
17.0 \\
\end{tabular} & \begin{tabular}{|l|}
78.4 \\
\end{tabular} & 0.17 & 5.9 & 1.0 & 254 & \begin{tabular}{|c|}
$<0.1$ \\
\end{tabular} & \begin{tabular}{|l|}
3.7 \\
\end{tabular} & \begin{tabular}{|l|}
0.4 \\
\end{tabular} & \begin{tabular}{|l|}
1.1 \\
\end{tabular} & 51 & \begin{tabular}{|l|}
0.4 \\
\end{tabular} & \begin{tabular}{|l|}
5.7 \\
\end{tabular} & 42 & \begin{tabular}{|c|}
$<0.2$ \\
\end{tabular} & 1.32 \\
\hline SP-04-1-2B & 2004 & .49495 & -92.88618 & low/medium & 288 & 0.33 & 4.5 & 22.1 & 790 & 18.2 & 78.2 & 0.18 & 5.6 & 0.8 & 269 & $<0.1$ & 3.5 & 0.4 & 1.0 & 42 & 0.4 & 5.4 & 46 & $<0.2$ & 1.73 \\
\hline SP-04-1-3B & 2004 & 48.49508 & -92.88588 & high & 314 & 0.78 & 5.4 & 35.3 & 230 & 23.2 & 75.0 & 0.26 & 5.9 & 1.3 & 265 & $<0.1$ & 3.4 & 0.4 & 1.0 & 55 & 0.5 & 5.7 & 46 & $<0.2$ & 2.05 \\
\hline SP-04-2-1B & 2004 & 48.49599 & -92.88988 & unburned & 653 & 0.39 & 6.5 & 18.9 & 310 & 17.8 & 85.6 & 0.23 & 7.4 & 1.2 & 275 & $<0.1$ & 4.8 & 0.4 & 1.3 & 51 & 0.5 & 6.9 & 39 & $<0.2$ & 2.72 \\
\hline SP-04-2-2B & 2004 & 48.49617 & -92.88976 & low/medium & 293 & 0.34 & 5.1 & 14.7 & 510 & 17.9 & 86.1 & 0.19 & 6.1 & 0.8 & 266 & $<0.1$ & 4.4 & 0.4 & 1.1 & 45 & 0.4 & 5.7 & 37 & $<0.2$ & 2.49 \\
\hline P-04-2-3B & 004 & .49626 & -92.88962 & high & 265 & 0.32 & 4.4 & 13.1 & 230 & 16.0 & 77.1 & 0.16 & 5.6 & 0.8 & 263 & $<0.1$ & 3.5 & 0.4 & 1.0 & 41 & 0.4 & 6.1 & 24 & $<0.2$ & 1.26 \\
\hline SP-04-3-1B & 2004 & 48.49990 & -92.88320 & high & 285 & 0.28 & 4.0 & 15.0 & 290 & 16.1 & 83.4 & 0.14 & 5.2 & 0.7 & 270 & $<0.1$ & 3.4 & 0.3 & 0.9 & 41 & 0.3 & 5.1 & 31 & $<0.2$ & 1.50 \\
\hline SP-04-3-2B & 2004 & 48.49950 & -92.88380 & low/medium & 240 & 1.14 & 6.9 & 21.0 & 180 & 20.8 & 61.6 & 0.34 & 6.5 & 1.5 & 213 & $<0.1$ & 5.8 & 0.3 & 4.5 & 76 & 0.6 & 7.4 & 47 & 0.4 & 2.60 \\
\hline SP-04-3-3B & 2004 & 48.49950 & -92.88430 & low/ub & 219 & 0.75 & 5.4 & 12.0 & 130 & 16.8 & 65.6 & 0.23 & 5.0 & 1.2 & 230 & $<0.1$ & 3.3 & 0.3 & 1.7 & 49 & 0.6 & 5.0 & 24 & $<0.2$ & 2.10 \\
\hline SP-04-4-1B & 2004 & 49866 & \begin{tabular}{|l|l} 
& -92.89069
\end{tabular} & unburned & 253 & 0.98 & 6.9 & 21.2 & 400 & 20.1 & 70.4 & 0.32 & 6.5 & 2.1 & 189 & $<0.1$ & 4.2 & 0.3 & 1.3 & 66 & 0.6 & 5.8 & 51 & $<0.2$ & 4.75 \\
\hline SP-04-4-1B dup & 2004 & 3.49866 & \begin{tabular}{|l|l} 
& -92.89069 \\
\end{tabular} & unburned & 251 & 0.95 & 7.0 & 23.4 & 400 & 21.2 & 68.2 & 0.31 & 6.3 & 1.4 & 187 & $<0.1$ & 4.3 & 0.3 & 1.3 & 65 & 0.6 & 6.0 & 65 & 0.3 & 4.93 \\
\hline SP-04-4-3B & 2004 & 48.49830 & -92.89090 & high & 245 & 0.45 & 5.0 & 17.7 & 180 & 17.0 & 82.8 & 0.18 & 5.6 & 0.9 & 252 & $<0.1$ & 4.5 & 0.3 & 1.0 & 46 & 0.4 & 5.0 & 33 & $<0.2$ & 1.60 \\
\hline SP-04-5-1B & 2004 & 48.50055 & \begin{tabular}{|l|l} 
& -92.88857 \\
\end{tabular} & unburned & 255 & 0.33 & 3.8 & 13.8 & 180 & 18.0 & 100.0 & 0.15 & 5.3 & 0.8 & 299 & $<0.1$ & 5.9 & 0.4 & 1.2 & 38 & 0.3 & 5.4 & 28 & $<0.2$ & 1.73 \\
\hline SP-04-5-2B & 2004 & 48.50060 & -92.88860 & low/medium & 288 & 0.25 & 3.6 & 11.4 & 110 & 14.4 & 86.9 & 0.12 & 5.4 & 0.6 & 277 & $<0.1$ & 3.7 & 0.3 & 0.8 & 35 & 0.2 & 5.4 & 21 & $<0.2$ & 0.87 \\
\hline SP-04-5-3B & 2004 & 3.50060 & -92.88840 & high & 289 & 0.35 & 4.1 & 13.1 & 90 & 15.4 & 75.2 & 0.17 & 5.5 & 0.7 & 287 & $<0.1$ & 3.9 & 0.3 & 1.0 & 41 & 0.3 & 5.6 & 25 & $<0.2$ & 1.32 \\
\hline SP-04-6-1B & 2004 & 48.50142 & -92.88990 & unburned & 739 & 0.59 & 7.0 & 21.7 & 1,830 & 19.8 & 74.0 & 1.56 & 6.9 & 66.1 & 187 & $<0.1$ & 5.1 & 0.3 & 2.3 & 66 & 0.7 & 6.0 & 82 & 0.4 & 5.75 \\
\hline SP-04-6-2B & 2004 & 48.50170 & $\mid-92.89010$ & low/medium & 254 & 1.12 & 7.2 & 16.7 & 510 & 20.7 & 72.2 & 0.28 & 5.8 & 1.4 & 208 & $<0.1$ & 4.4 & 0.4 & 2.0 & 69 & 0.7 & 6.2 & 38 & 0.4 & 3.24 \\
\hline SP-04-6-3B & 2004 & 48.50180 & $\mid-92.89100$ & high & 229 & 0.39 & 4.4 & 9.0 & 260 & 16.0 & 70.3 & 0.17 & 4.5 & 0.8 & 251 & $<0.1$ & 3.4 & 0.3 & 0.9 & 37 & 0.4 & 4.8 & 22 & $<0.2$ & 1.60 \\
\hline SP-04-6-3B dup & 2 & .50180 & $\mid-92.8$ & high & 245 & 0.42 & 4.5 & 8.6 & 270 & 16.7 & 70.0 & 0.18 & 4.6 & 1.0 & 247 & $<0.1$ & 3.1 & 0.4 & 1.1 & 36 & 0.4 & 5.0 & 23 & $<0.2$ & 1.80 \\
\hline SP-04-7-1B & 2004 & 48.50540 & -92.898 & unburned & 210 & 0.78 & 6.8 & 18.4 & 210 & 17.9 & 76.7 & 0.22 & 6.3 & 1.5 & 219 & $<0.1$ & 3.9 & 0.5 & 9.2 & 57 & 0.6 & 5.5 & 28 & 0.3 & 2.00 \\
\hline SP-04-7-2B & 2004 & 48.50501 & $\mid-92.8$ & low/medium & 494 & 1.25 & 7.5 & 32.7 & 950 & 19.7 & 77.9 & 0.34 & 7.0 & 1.5 & 159 & $<0.1$ & 6.0 & 0.4 & 8.2 & 81 & 0.9 & 6.7 & 93 & 0.6 & 4.79 \\
\hline SP-04-7-3B & 2004 & 48.50480 & -92.89560 & high & 257 & 0.26 & 3.3 & 13.0 & 210 & 16.0 & 83.5 & 0.18 & 4.9 & 0.8 & 273 & $<0.1$ & 3.3 & 0.3 & 0.8 & 32 & 0.3 & 5.1 & 29 & $<0.2$ & 1.29 \\
\hline SP-04-8-1B & 2004 & 48.50166 & -92.87214 & unburned & 1,340 & 0.61 & 5.0 & 19.4 & 500 & 22.0 & 65.3 & 0.31 & 6.1 & 1.1 & 255 & $<0.1$ & 3.8 & 0.3 & 1.0 & 51 & 0.4 & 6.0 & 64 & 0.2 & 3.28 \\
\hline SP-04-8-2B & 2004 & 48.50170 & -92.87230 & low/medium & 356 & 0.71 & 8.0 & 25.3 & 480 & 19.4 & 87.2 & 0.29 & 8.6 & 1.6 & 207 & $<0.1$ & 6.7 & 0.5 & 3.9 & 85 & 0.7 & 6.5 & 50 & 0.3 & 2.34 \\
\hline SP-04 & & & $\mid-92.8$ & low/me & 356 & 0.87 & 7.8 & 29.2 & 500 & 19.8 & 84.9 & 0.30 & 8.5 & 1.5 & 211 & $<0.1$ & 5.0 & 0.6 & 3.8 & 85 & 0.8 & 6.2 & 53 & 0.3 & 2.37 \\
\hline SP-04-8-3B & 200 & 48.50180 & -92.87250 & high & 257 & 1.23 & 6.1 & 28.7 & 500 & 18.1 & 64.5 & 0.30 & 7.1 & 1.0 & 235 & $<0.1$ & 5.1 & 0.4 & 2.7 & 69 & 0.6 & 5.5 & 44 & 0.7 & 3.54 \\
\hline SP-04-9-1B & 2004 & 48.50080 & -92.87210 & unburn & 330 & 0.32 & 4.6 & 11.3 & 300 & 18.6 & 82.7 & $0.1 \varepsilon$ & 5.2 & 0.8 & 258 & $<0.1$ & 3.4 & 0.3 & 0.9 & 35 & 0.4 & 5.3 & 30 & $<0.2$ & 1.57 \\
\hline SP-04-9- & 2004 & 48.50097 & \begin{tabular}{|l|l}
-92.87247 \\
\end{tabular} & low/medium & 424 & 0.47 & 6.6 & 19.2 & 430 & 18.2 & 94.8 & 0.23 & 7.3 & 1.1 & 252 & $<0.1$ & 5.0 & 0.4 & 1.2 & 58 & 0.6 & 6.3 & 37 & $<0.2$ & 1.94 \\
\hline SP-C & & 50130 & $\mid-92.8$ & high & 336 & 0.71 & 5.6 & 28.6 & 960 & 20.1 & 66.3 & 0.25 & 7.1 & 0.9 & 254 & $<0.1$ & 3.9 & 0.3 & 1.2 & 70 & 0.6 & 5.6 & 53 & 0.3 & 2.98 \\
\hline SP-04-10-1B & 2004 & 48.50210 & $\mid-92.88490$ & unburned & 3,400 & 0.87 & 6.0 & 23.8 & 1,110 & 20.1 & 87.7 & 0.27 & 6.7 & 1.1 & 253 & $<0.1$ & 4.5 & 0.5 & 1.3 & 61 & 0.6 & 6.7 & 141 & 0.3 & 2.82 \\
\hline SP-04-10-2B & 2004 & 48.50192 & -92.88459 & low/medium & 336 & 0.61 & 5.3 & 16.9 & 270 & 18.2 & 77.3 & 0.20 & 6.2 & 0.8 & 268 & $<0.1$ & 3.0 & 0.4 & 1.0 & 53 & 0.4 & 5.6 & 49 & 0.2 & 1.73 \\
\hline SP-04-10-3B & 2004 & 48.50180 & -92.88450 & high & 241 & 0.57 & 4.8 & 12.4 & 130 & 17.3 & 75.2 & 0.23 & 5.2 & 1.0 & 256 & $<0.1$ & 3.1 & 0.3 & 0.9 & 43 & 0.5 & 5.6 & 29 & 0.2 & 1.78 \\
\hline & & 3.49490 & $\mid-92.88660$ & unburn & 194 & 0.18 & 4.6 & 6.0 & 150 & 14.5 & 67.8 & 0.13 & 3.5 & 1.5 & 236 & $<0.1$ & 3.3 & 0.3 & 1.1 & 24 & 0.3 & 5.5 & 13 & 0.2 & 1.49 \\
\hline SP-05-1-2B & 2005 & 3.49495 & \begin{tabular}{|l|l} 
& -92.88618 \\
\end{tabular} & low $/ \mathrm{m}$ & 212 & 0.20 & 3.6 & 9.1 & 480 & 16.3 & 75.0 & 0.15 & 4.4 & 3.4 & 237 & $<0.1$ & 2.7 & 0.3 & 0.9 & 32 & 0.3 & 4.7 & 25 & $<0.2$ & 1.80 \\
\hline SP-05-1-3B & 2005 & 48.49508 & -92.88588 & high & 381 & 0.52 & 4.7 & 13.1 & 290 & 19.6 & 75.4 & 0.20 & 4.7 & 4.7 & 225 & $<0.1$ & 3.1 & 0.4 & 1.0 & 48 & 0.4 & 5.5 & 32 & $<0.2$ & 2.28 \\
\hline SP-05-2-1B & 2005 & 48.49599 & \begin{tabular}{|l|l} 
& -92.88988 \\
\end{tabular} & |unburned & 441 & 0.34 & 5.6 & 17.0 & 310 & 15.9 & 78.6 & 0.23 & 7.0 & 5.7 & 234 & $<0.1$ & 4.0 & 0.4 & 1.2 & 60 & 0.6 & 6.3 & 37 & $<0.2$ & 2.69 \\
\hline & 2 & 3.49617 & -92.88976 & low/medium & 245 & 0.20 & 3.9 & 8.1 & 220 & 16.6 & 71.4 & 0.17 & 4.7 & 2.3 & 245 & $<0.1$ & 6.3 & 0.4 & 1.0 & 34 & 0.5 & 5.4 & 22 & $<0.2$ & 2.85 \\
\hline SP-05-2-3B & & 49626 & & high & 250 & 0.32 & 4.5 & 13.4 & 310 & 16.3 & 72.2 & 0.16 & 5.2 & 2.2 & 238 & $<0.1$ & 3.2 & 0.4 & 1.0 & 47 & 0.4 & 5.8 & & 0.6 & 1.29 \\
\hline SP-05-2-3B dup & 2005 & 48.49626 & -92.88962 & high & 248 & 0.31 & 6.2 & 12.8 & 320 & 16.3 & 73.8 & 0.14 & 5.3 & 0.8 & 250 & $<0.1$ & 3.7 & 0.4 & 1.1 & 49 & 0.4 & 5.5 & 24 & $<0.2$ & 1.21 \\
\hline SP-05-3-1B & 2005 & 48.49990 & -92.88320 & high & 208 & 0.27 & 3.8 & 12.6 & 280 & 16.7 & 74.7 & 0.20 & 4.4 & 4.4 & 240 & $<0.1$ & 3.0 & 0.4 & 0.9 & 33 & 0.4 & 5.9 & 28 & 0.4 & 2.86 \\
\hline SP-05-3-2B & 2005 & 48.49950 & $\mid-92.88380$ & low/medium & 198 & 1.17 & 5.8 & 16.4 & 300 & 21.1 & 51.1 & 0.29 & 5.1 & 1.6 & 187 & $<0.1$ & 3.7 & 0.3 & 6.1 & 70 & 0.5 & 6.6 & 35 & 0.6 & 5.50 \\
\hline SP-05-3-3B & 2005 & 48.49950 & -92.88430 & unburned & 195 & 0.93 & 5.4 & 12.2 & 210 & 18.4 & 62.4 & 0.36 & 4.9 & 3.6 & 198 & $<0.1$ & 3.5 & 0.4 & 3.5 & 58 & 0.5 & 5.4 & 28 & 0.4 & 4.39 \\
\hline SP-05-4-1B & 2005 & 48.49866| & | -92.89069 & unburned & 197 & 1.07 & 6.3 & 13.4 & 440 & 18.7 & 64.5 & 0.27 & 5.1 & 2.3 & 159 & $<0.1$ & 4.3 & 0.4 & 1.5 & 59 & 0.6 & 5.8 & 43 & 0.3 & 4.68 \\
\hline
\end{tabular}




\begin{tabular}{|c|c|c|c|c|c|c|c|c|c|c|c|c|c|c|c|c|c|c|c|c|c|c|c|c|c|}
\hline Field no. & $\begin{array}{c}\text { Year } \\
\text { collected }\end{array}$ & Latitude & Longitude & Fire severity & $\begin{array}{c}\text { Mn } \\
(\mathrm{ppm})\end{array}$ & $\begin{array}{c}\text { Mo } \\
\text { (ppm) }\end{array}$ & $\begin{array}{c}\mathrm{Nb} \\
(\mathrm{ppm})\end{array}$ & $\begin{array}{c}\mathrm{Ni} \\
(\mathrm{ppm})\end{array}$ & $\begin{array}{c}P \\
(\mathrm{ppm})\end{array}$ & \begin{tabular}{|c|}
$\mathrm{Pb}$ \\
$(\mathrm{ppm})$
\end{tabular} & $\begin{array}{c}\mathrm{Rb} \\
\text { (ppm) }\end{array}$ & \begin{tabular}{|c|}
$\mathrm{Sb}$ \\
$(\mathrm{ppm})$
\end{tabular} \mid & $\begin{array}{c}\text { Sc } \\
\text { (ppm) }\end{array}$ & $\begin{array}{c}\mathrm{Sn} \\
(\mathrm{ppm})\end{array}$ & $\begin{array}{c}\mathrm{Sr} \\
(\mathrm{ppm})\end{array}$ & \begin{tabular}{|c|}
$\mathrm{Te}$ \\
$(\mathrm{ppm})$
\end{tabular} & \begin{tabular}{|c|} 
Th \\
$(\mathrm{ppm})$
\end{tabular} & \begin{tabular}{|c|}
$\mathrm{TI}$ \\
$(\mathrm{ppm})$
\end{tabular} & \begin{tabular}{|c|}
$U$ \\
$(\mathrm{ppm})$ \\
\end{tabular} & \begin{tabular}{|c|}
$\mathrm{V}$ \\
$(\mathrm{ppm})$
\end{tabular} & \begin{tabular}{|c|} 
W \\
$(\mathrm{ppm})$
\end{tabular} & \begin{tabular}{|c|}
$Y$ \\
$(\mathrm{ppm})$
\end{tabular} & \begin{tabular}{|c|}
$\mathrm{Zn}$ \\
(ppm)
\end{tabular} & \begin{tabular}{|c|} 
Se \\
$(\mathrm{ppm})$
\end{tabular} & $\begin{array}{l}\text { Total C } \\
\text { (wt. \%) }\end{array}$ \\
\hline SP-05-4-1B dup & 2005 & 48.49866 & -92.89069 & unburned & 204 & 1.02 & \begin{tabular}{|l|}
6.5 \\
\end{tabular} & 14.2 & 450 & \begin{tabular}{|l|}
19.6 \\
\end{tabular} & \begin{tabular}{|l|l}
63.7 \\
\end{tabular} & \begin{tabular}{|l|}
0.26 \\
\end{tabular} & \begin{tabular}{|l|}
5.2 \\
\end{tabular} & 1.3 & 166 & $\begin{array}{l}<0.1 \\
\end{array}$ & 4.4 & \begin{tabular}{|l|}
0.4 \\
\end{tabular} & 1.6 & \begin{tabular}{|l|}
62 \\
\end{tabular} & 0.6 & \begin{tabular}{|l|}
5.8 \\
\end{tabular} & 44 & \begin{tabular}{|l|}
0.3 \\
\end{tabular} & 4.52 \\
\hline SP-05-4-2B & 2005 & 48.49850 & -92.89060 & low/medium & 198 & 1.19 & 8.6 & 12.4 & 1,020 & 21.4 & 59.9 & 0.32 & 6.5 & 4.4 & 149 & $<0.1$ & 4.8 & 0.4 & 5.9 & 72 & 1.0 & 6.3 & 50 & 0.3 & 4.52 \\
\hline P-05-4-3B & 2005 & .49830 & -92.89090 & |high & 208 & 0.34 & 4.1 & 16.1 & 160 & 15.3 & 73.5 & 0.16 & 4.6 & 0.9 & 225 & $<0.1$ & 4.1 & 0.4 & 1.1 & 44 & 0.4 & 5.5 & 25 & 0.2 & 1.88 \\
\hline SP-05-5-1B & 2005 & 48.50055 & -92.88857 & unburned & 277 & 0.12 & 2.1 & 14.0 & 210 & 11.3 & 45.4 & 0.11 & 3.0 & 0.7 & 151 & $<0.1$ & 12.7 & 0.2 & 0.6 & 22 & 0.2 & 3.3 & 19 & $<0.2$ & 2.69 \\
\hline SP-05-5-2B & 2005 & 48.50060 & -92.88860 & low/medium & 220 & 0.37 & 3.4 & 14.8 & 160 & 14.2 & 77.6 & 0.12 & 4.6 & 1.0 & 241 & $<0.1$ & 5.3 & 0.4 & 0.9 & 36 & 0.3 & 5.0 & 21 & $<0.2$ & 2.02 \\
\hline SP-05-5-3B & 2005 & 48.50060 & $\mid-92.88840$ & |high & 206 & 0.19 & 3.1 & 8.5 & 120 & 13.7 & 67.0 & 0.14 & 4.2 & 1.5 & 254 & $<0.1$ & 2.8 & 0.3 & 0.8 & 30 & 0.4 & 4.7 & 16 & $<0.2$ & 1.62 \\
\hline ?-05-6-1B & 2005 & .50142 & -92.88990 & unburned & 487 & 0.60 & 6.9 & 17.4 & 1,690 & 18.9 & 67.9 & 0.27 & 5.8 & 3.1 & 167 & $<0.1$ & 4.9 & 0.4 & 2.3 & 62 & 0.7 & 5.8 & 74 & 0.4 & 5.01 \\
\hline SP-05-6-2B & 2005 & 48.50170 & \begin{tabular}{|l|l} 
& -92.89010
\end{tabular} & low/medium & 453 & 0.81 & 6.8 & 10.6 & 520 & 22.6 & 62.4 & 0.30 & 4.9 & 1.8 & 176 & $<0.1$ & 3.8 & 0.4 & 1.6 & 56 & 0.7 & 6.0 & 42 & 0.3 & 5.25 \\
\hline SP-05-6-3B & 2005 & 48.50180 & -92.89100 & high & 207 & 0.44 & 4.3 & 11.4 & 360 & 14.2 & 73.9 & 0.15 & 4.3 & 0.9 & 229 & $<0.1$ & 2.8 & 0.4 & 0.9 & 42 & 0.4 & 4.8 & 19 & $<0.2$ & 1.77 \\
\hline SP-05-7-1B & 2005 & 48.50540 & -92.89810 & |unburned & 172 & 0.70 & 5.3 & 13.7 & 230 & 16.3 & 72.1 & 0.17 & 5.3 & 1.3 & 205 & $<0.1$ & 3.3 & 0.4 & 8.1 & 49 & 0.5 & 4.9 & 22 & $<0.2$ & 2.93 \\
\hline SP-05-7-2B & 2005 & 48.50501 & \begin{tabular}{|l|l} 
& -92.89789
\end{tabular} & low/medium & 420 & 1.44 & 7.4 & 35.6 & 1,120 & 18.9 & 71.2 & 0.32 & 5.9 & 1.7 & 136 & $<0.1$ & 8.8 & 0.5 & 11.1 & 85 & 0.9 & 6.5 & 81 & 0.8 & 6.89 \\
\hline P-05-7-3B & 2005 & 48.50480 & -92.89560 & high & 233 & 0.38 & 3.6 & 21.0 & 310 & 15.9 & 77.5 & 0.15 & 4.8 & 1.6 & 263 & $<0.1$ & 2.8 & 0.4 & 0.9 & 37 & 0.4 & 4.8 & 24 & $<0.2$ & 1.84 \\
\hline SP-05-8-1B & 2005 & 48.50166 & $\mid-92.87214$ & unburned & 433 & 0.49 & 5.5 & 17.2 & 570 & 19.7 & 65.2 & 0.21 & 5.5 & 1.0 & 221 & $<0.1$ & 3.4 & 0.4 & 1.2 & 60 & 0.5 & 5.1 & 61 & 0.2 & 3.28 \\
\hline SP-05-8-2B & 2005 & 48.50170 & -92.87230 & low/medium & 250 & 0.83 & 8.7 & 22.9 & 680 & 21.5 & 74.6 & 0.26 & 7.9 & 1.7 & 164 & $<0.1$ & 5.1 & 0.5 & 4.5 & 91 & 0.7 & 6.2 & 57 & 0.3 & 2.96 \\
\hline SP-05-8-3B & 2005 & 48.50180 & -92.87250 & high & 222 & 1.12 & 6.2 & 21.4 & 560 & 16.9 & 57.4 & 0.30 & 6.5 & 1.1 & 208 & $<0.1$ & 5.0 & 0.4 & 2.1 & 76 & 0.6 & 5.3 & 46 & 0.5 & 4.69 \\
\hline SP-05-9-1B & 2005 & 48.50080 & $\mid-92.87210$ & unburned & 554 & 0.26 & 3.9 & 7.8 & 310 & 16.5 & 81.7 & 0.16 & 4.5 & 0.7 & 232 & $<0.1$ & 2.4 & 0.4 & 0.9 & 33 & 0.3 & 4.5 & 28 & $<0.2$ & 1.86 \\
\hline SP-05-9-1B dup & 2005 & 48.50080 & -92.87210 & |unburned & 462 & 0.23 & 3.7 & 7.5 & 300 & 15.5 & 82.5 & 0.14 & 4.2 & 0.7 & 228 & $<0.1$ & 2.4 & 0.4 & 0.8 & 32 & 0.3 & 4.6 & 27 & $<0.2$ & 1.72 \\
\hline SP-05-9-2B & 2005 & 48.50097 & -92.87247 & low/medium & 393 & 0.36 & 6.3 & 13.6 & 470 & 18.8 & 96.1 & 0.22 & 6.6 & 1.3 & 242 & $<0.1$ & 4.0 & 0.5 & 1.3 & 59 & 0.5 & 5.9 & 36 & $<0.2$ & 2.56 \\
\hline SP-05-9-3E & 200 & 48.50130 & -92.87270 & high & 325 & 0.36 & 5.0 & 8.9 & 440 & 18.4 & 72.1 & 0.20 & 4.7 & 1.0 & 227 & $<0.1$ & 2.9 & 0.4 & 1.1 & 41 & 0.8 & 4.8 & 42 & $<0.2$ & 2.56 \\
\hline SP-05-10-1B & 2005 & 48.50210 & -92.88490 & unburned & 1,530 & 0.60 & 6.0 & 19.5 & 890 & 17.3 & 82.7 & 0.23 & 7.3 & 1.2 & 244 & $<0.1$ & 4.4 & 0.4 & 1.4 & 69 & 0.6 & 6.8 & 89 & 0.2 & 2.13 \\
\hline SP-05-10-2B & 2005 & 48.50192 & -92.88459 & low/medium & 297 & 0.39 & 4.3 & 11.8 & 250 & 15.6 & 79.2 & 0.17 & 5.3 & 0.7 & 243 & $<0.1$ & 3.9 & 0.4 & 0.9 & 45 & 0.4 & 4.9 & 41 & $<0.2$ & 1.90 \\
\hline SP-05-10-3B & 2005 & 48.50180 & -92.88450 & |high & 182 & 0.28 & 3.8 & 5.8 & 90 & 13.8 & 72.9 & 0.15 & 4.1 & 0.7 & 244 & $<0.1$ & 2.7 & 0.5 & 1.0 & 31 & 0.3 & 4.7 & 18 & $<0.2$ & 2.14 \\
\hline SP-06 1-1B & 2006 & 48.49490 & -92.88660 & |unburned & 272 & 0.41 & 3.6 & 9.3 & 210 & 14.4 & 78.6 & 0.11 & 4.7 & 0.7 & 266 & $<0.1$ & 4.4 & 0.4 & 1.0 & 32 & 0.4 & 5.7 & 23 & $<0.2$ & 1.33 \\
\hline SP-06 1-2B & 2006 & 48.49495 & -92.88618 & low/medium & 299 & 0.32 & 3.3 & 16.6 & 720 & 15.3 & 75.2 & 0.0 & 5.2 & 0.7 & 264 & $<0.1$ & 3.4 & 0.4 & 1.0 & 38 & 0.3 & 5.6 & 27 & $<0.2$ & 1.60 \\
\hline SP-06 1-3B & 2006 & 48.49508 & -92.885 & high & 265 & 0.90 & 4.6 & 21.2 & 440 & 15.5 & 74.8 & 0.11 & 6.0 & 0.8 & 244 & $<0.1$ & 3.8 & 0.4 & 1.1 & 59 & 0.4 & 5.8 & 31 & 0.2 & 1.54 \\
\hline SP-06 2-1B & 2006 & 48.49599 & -92.889 & unburn & 615 & 0.55 & 5.7 & 21.2 & 380 & 16.1 & 86.3 & 0.15 & 7.9 & 2.0 & 254 & $<0.1$ & 5.0 & 0.5 & 1.3 & 63 & 0.5 & 7.2 & 56 & $<0.2$ & 2.22 \\
\hline SP-06 2-2B & 2006 & 48.49617 & -92.88976 & low/medium & 375 & 0.36 & 4.3 & 14.4 & 510 & 15.1 & 82.2 & 0.11 & 6.3 & 0.8 & 262 & $<0.1$ & 3.7 & 0.4 & 1.1 & 46 & 0.4 & 6.5 & 33 & $<0.2$ & 1.75 \\
\hline SP-06 2-3B & 2006 & 48.49626 & $\mid-92.88962$ & high & 288 & 0.60 & 4.1 & 21.8 & 570 & 14.6 & 74.5 & 0.12 & 6.5 & 0.8 & 259 & $<0.1$ & 4.2 & 0.4 & 1.0 & 55 & 0.4 & 6.1 & 31 & $<0.2$ & 1.38 \\
\hline SP-06 3-1B & 2006 & 48.49990 & $\mid-92.88320$ & high & 313 & 0.41 & 4.0 & 16.3 & 260 & 14.3 & 85.0 & 0.07 & 5.9 & 0.7 & 284 & $<0.1$ & 3.5 & 0.4 & 1.0 & 43 & 0.3 & 6.2 & 23 & $<0.2$ & 1.14 \\
\hline BB & & & $\mid-92.88380$ & low/me & 247 & 1.41 & 5.3 & 17.1 & 260 & 17.9 & & & 5.9 & 1.3 & 211 & $<0.1$ & 4.5 & 0.3 & 3.8 & 72 & 0.4 & 6.7 & 3 & 0.3 & 3.09 \\
\hline SP-06 3-3B & 2006 & 48.49950 & -92.88430 & unburned & 256 & 1.76 & 5.2 & 16.7 & 290 & 17.2 & 68.4 & 0.1 & 5.8 & 1.1 & 228 & $<0.1$ & 4.9 & 0.4 & 3.8 & 72 & 0.4 & 6.4 & 30 & 0.3 & 2.11 \\
\hline SP-06 4-1B & 2006 & 48.49866 & \begin{tabular}{|l|l} 
& -92.89069
\end{tabular} & unburned & 1,260 & 1.29 & 5.2 & 17.0 & 360 & 20.0 & 78.6 & 0.17 & 6.3 & 1.3 & 233 & $<0.1$ & 5.4 & 0.5 & 1.9 & 63 & 0.5 & 6.2 & 64 & 0.3 & 2.28 \\
\hline SP-06 4-1B dup & 2006 & 48.49866 & \begin{tabular}{|l|l} 
& -92.89069
\end{tabular} & unburned & 1,070 & 1.14 & 4.6 & 16.6 & 330 & 18.7 & 79.0 & 0.17 & 5.9 & 1.0 & 237 & $<0.1$ & 4.0 & 0.5 & 1.6 & 59 & 0.3 & 5.1 & 38 & 0.3 & 2.08 \\
\hline SP-06 4-2B & 2006 & 48.49850 & -92.89060 & low/medium & 206 & 1.02 & 5.7 & 8.4 & 650 & 23.9 & 60.5 & & 5.7 & 2.2 & 204 & $<0.1$ & 5.0 & 0.4 & 6.2 & 43 & 0.7 & 6.2 & 34 & 0.4 & 5.35 \\
\hline SP-06 4-3B & 2006 & 48.49830 & \begin{tabular}{|l|l} 
& -92.89090
\end{tabular} & |high & 242 & 0.40 & 3.6 & 14.6 & 170 & 14.6 & 78.5 & & 5.2 & 0.6 & 248 & $<0.1$ & 3.6 & 0.4 & 1.0 & 45 & 0.2 & 5.3 & 24 & $<0.2$ & 1.38 \\
\hline SP-06 5-1B & 2006 & 48.50055 & \begin{tabular}{|l|l} 
& -92.88857
\end{tabular} & unburned & 400 & 0.41 & 3.3 & 10.5 & 330 & 16.4 & 78.0 & 0.07 & 4.7 & 0.5 & 244 & $<0.1$ & 3.9 & 0.4 & 0.8 & 33 & 0.3 & 5.3 & 27 & $<0.2$ & 2.26 \\
\hline SP-06 5-2B & 2006 & 48.50060 & -92.88860 & low/medium & 259 & 0.27 & 2.9 & 8.5 & 150 & 13.1 & 85.2 & 0.05 & 4.6 & 0.6 & 258 & $<0.1$ & 2.8 & 0.4 & 0.8 & 33 & 0.2 & 5.1 & 17 & $<0.2$ & 0.74 \\
\hline SP-06 5-3B & 2006 & 48.50060 & \begin{tabular}{|l|l} 
& -92.88840
\end{tabular} & high & 287 & 0.41 & 3.1 & 12.3 & 120 & 14.1 & 77.1 & 0.06 & 5.5 & 0.6 & 278 & $<0.1$ & 3.0 & 0.4 & 0.9 & 38 & 0.2 & 5.6 & 19 & $<0.2$ & 1.68 \\
\hline SP-06 6-1B & 2006 & 48.50142 & -92.88990 & unburned & 332 & 0.65 & 3.7 & 20.0 & 360 & 16.2 & 75.8 & & 5.6 & 0.7 & 25 & $<0.1$ & 3.5 & 0.4 & 1.0 & 51 & 0.4 & 5.4 & & $<0.2$ & 1.68 \\
\hline SP-06 6-2B & 2006 & 48.50170 & -92.89010 & low/medium & 279 & 0.98 & 4.9 & 22.5 & 620 & 16.6 & 74.8 & 0.16 & 5.9 & 1.0 & 228 & $<0.1$ & 4.3 & 0.4 & 1.6 & 59 & 0.5 & 5.9 & 36 & 0.3 & 2.72 \\
\hline SP-06 6-2B dup & 2006 & 48.50170 & \begin{tabular}{|l|l} 
& -92.89010
\end{tabular} & low/medium & 284 & 0.75 & 4.8 & 15.2 & 590 & 19.8 & 71.6 & 0.19 & 5.6 & 1.1 & 214 & $<0.1$ & 4.5 & 0.4 & 1.4 & 58 & 0.4 & 5.2 & 35 & 0.2 & 2.92 \\
\hline SP-06 6-3B & 2006 & 48.50180 & \begin{tabular}{|l|l} 
& -92.89100
\end{tabular} & high & 238 & 0.81 & 5.0 & 12.1 & 340 & 18.3 & 67.9 & 0.20 & 4.4 & 0.9 & 224 & $<0.1$ & 3.3 & 0.5 & 1.1 & 46 & 0.5 & 5.6 & 26 & 0.2 & 2.20 \\
\hline SP-06 7-1B & 2006 & 48.50540 & | -92.89810 & unburned & 263 & 1.03 & 5.2 & 16.8 & 330 & 16.6 & 77.6 & 0.12 & 6.3 & 1.1 & 205 & $<0.1$ & 5.1 & 0.5 & 8.6 & 70 & 0.4 & 6.5 & 31 & 0.3 & 2.42 \\
\hline
\end{tabular}


Appendix 5-B Horizon Chemistry, Shoepack Lake, Voyageurs National Park, MN

\begin{tabular}{|c|c|c|c|c|c|c|c|c|c|c|c|c|c|c|c|c|c|c|c|c|c|c|c|c|c|c|}
\hline Field no. & \begin{tabular}{|c} 
Year \\
collected
\end{tabular} & Latitude & Longitude & Fire severity & $\begin{array}{c}\mathrm{Mn} \\
(\mathrm{ppm})\end{array}$ & \begin{tabular}{|c|} 
Mo \\
(ppm)
\end{tabular} & $\begin{array}{l}\mathrm{Nb} \\
\text { (ppm) }\end{array}$ & $\begin{array}{c}\mathrm{Ni} \\
\text { (ppm) }\end{array}$ & $\begin{array}{c}P \\
(\mathrm{ppm})\end{array}$ & \begin{tabular}{|c}
$\mathrm{Pb}$ \\
$(\mathrm{ppm})$
\end{tabular} & \begin{tabular}{|c|}
$\mathrm{Rb}$ \\
(ppm)
\end{tabular} & $\begin{array}{l}\text { Sb } \\
\text { (ppm) }\end{array}$ & $\begin{array}{c}\text { Sc } \\
(\mathrm{ppm})\end{array}$ & $\begin{array}{c}\mathrm{Sn} \\
(\mathrm{ppm}\end{array}$ & $\begin{array}{c}\mathrm{Sr} \\
(\mathrm{ppm}\end{array}$ & \begin{tabular}{|c}
$\mathrm{Te}$ \\
(ppm
\end{tabular} & $\begin{array}{l}\text { Th } \\
\text { (ppm) }\end{array}$ & $\begin{array}{c}\mathrm{Tl} \\
(\mathrm{ppm}\end{array}$ & $\underset{(\mathrm{ppm}}{\mathrm{U}}$ & $\begin{array}{c}\mathrm{V} \\
\text { (ppm }\end{array}$ & $\begin{array}{r}\text { W } \\
\text { (pp }\end{array}$ & & $\begin{array}{c}\mathrm{Y} \\
\mathrm{opm})\end{array}$ & $\begin{array}{c}\mathrm{Zn} \\
(\mathrm{ppm})\end{array}$ & \begin{tabular}{|c|} 
Se \\
(ppm)
\end{tabular} & $\begin{array}{l}\text { Total C } \\
\text { (wt. \%) }\end{array}$ \\
\hline $667-2 B$ & 2006 & 48.50501 & -92.89789 & low/medium & 390 & 1.26 & 5.4 & 26.9 & 710 & 17. & 76.0 & 0.22 & 6.9 & 1 & 18 & $<0$ & 5.1 & 0. & 5. & 7 & & & 6.1 & 81 & 0.5 & 3.29 \\
\hline 66 7-3B & 200 & & .89560 & high & 278 & 0.36 & 3.3 & 12.3 & 290 & 15.3 & 84.2 & 0.09 & 4.8 & 0. & 27 & $<0$. & 3.6 & 0. & 1. & 3 & & & 5.5 & 21 & $<0.2$ & 1.26 \\
\hline 66 8-1B & 2006 & 50166 & 14 & unb & 887 & 0.48 & 4.7 & 15.3 & 580 & 15.5 & 70.2 & 0.14 & 5.6 & $1 .($ & 24 & $<0$. & 3.6 & 0. & 1. & 5 & & & 6.2 & 56 & 0.2 & $2.0 \mathrm{~s}$ \\
\hline$-068-2 B$ & 2006 & & & low $/ \mathrm{m}$ & 366 & 0.88 & 6.3 & 19.3 & 460 & 18.6 & 74.8 & 0.18 & 7.6 & 1.2 & 19 & $<0$. & 5.1 & 0.5 & 3. & 7 & & & 6.7 & 49 & 0.2 & 1.85 \\
\hline-06 8-3B & 2006 & & & high & 268 & 1.06 & 5.0 & 15.2 & 720 & 18.8 & 72.9 & 0.21 & 6.6 & 1. & 20 & $<0$. & 5.2 & 0. & 2. & 6 & & & 6.0 & 55 & 0.3 & $2.7 \mathrm{~s}$ \\
\hline-06 8-3B d & 2006 & 50180 & -92.87250 & higl & 267 & 0.89 & 5.0 & 14.7 & 730 & 18.2 & 65.3 & 0.20 & 6.1 & 1.3 & 19 & $<0$. & 7. & 0. & 2. & 7 & & & 6.5 & 55 & 0.3 & $2.7 \mathrm{~s}$ \\
\hline -06 9-1B & 2006 & 50080 & -92.87210 & unburned & $27 \epsilon$ & 0.33 & 3.2 & 9.2 & 280 & 17.0 & 75.8 & 0.12 & 5.3 & 0. & 24 & $<0$. & 3.7 & 0. & 1. & 3 & & & 5.9 & 27 & $<0.2$ & 1.35 \\
\hline -06 9-2B & 2006 & 97 & -92.87247 & low/medium & 410 & 0.50 & 5.0 & 18.4 & 490 & 16.7 & 91.5 & 0.14 & 7.6 & 1.2 & 25 & $<0$. & 4.3 & 0. & 1. & 5 & & & 6.5 & 34 & $<0.2$ & 1.76 \\
\hline 069 & & & & high & 266 & 0.70 & 4.6 & 13.1 & 750 & 16.5 & 75.5 & 0.19 & 5.7 & 1. & 22 & $<0$. & 3.5 & 0.6 & 1. & 5 & & & 5.6 & 46 & $<0.2$ & 2.00 \\
\hline $6610-$ & 2006 & & & & 856 & 0.64 & 5.3 & 19.9 & 1,020 & 18.4 & 71.9 & 0.18 & 7. & $1 .(\mathrm{r}+\mathrm{r})$ & 228 & $<0$. & Ј. & $0 .{ }^{\circ}$ & 1. & & & & 6.3 & 90 & 0.2 & 1.68 \\
\hline 6 10-2B & 2006 & 50192 & -92.88459 & low/medium & 926 & 0.78 & 4.4 & 14.6 & 340 & 20.5 & 80.7 & 0.25 & 6.0 & 0. & 24 & $<0$. & 4. & 0. & 1. & & & & 5.8 & 45 & 0.2 & 2.06 \\
\hline$-0610-3 B$ & 2006 & 48.50180 & -92.88450 & high & 240 & 0.51 & 3.5 & 10.5 & 140 & 14.6 & 70.2 & 0.14 & 5.2 & 0.7 & 25 & $<0$. & 3.7 & 0. & 1. & 4 & 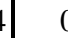 & & 5.2 & 23 & $<0.2$ & 0.97 \\
\hline
\end{tabular}


Click back to Contents

Appendix 6-O Horizon Chemistry, Shoepack Lake, Voyageurs National Park, MN

\begin{tabular}{|c|c|c|c|c|c|c|c|c|c|c|c|c|c|c|c|c|c|c|c|c|c|c|c|c|c|c|c|c|}
\hline Field no. & $\begin{array}{c}\text { Year } \\
\text { collected }\end{array}$ & Latitude & Longitude & Fire severity & $\begin{array}{c}\text { Ash } \\
\%\end{array}$ & $\begin{array}{c}\mathrm{Al} \\
\text { (wt. \%) }\end{array}$ & \begin{tabular}{|c|}
$\mathrm{Ca}$ \\
(wt. \%)
\end{tabular} & $\begin{array}{c}\mathrm{Fe} \\
\text { (wt. \%) }\end{array}$ & $\begin{array}{c}\mathrm{K} \\
\text { (wt. \%) }\end{array}$ & $\begin{array}{c}\mathrm{Mg} \\
\text { (wt. \%) }\end{array}$ & $\begin{array}{c}\mathrm{Na} \\
\text { (wt. \%) }\end{array}$ & $\begin{array}{c}\mathrm{S} \\
\text { (wt. \%) }\end{array}$ & $\begin{array}{c}\mathrm{Ti} \\
\text { (wt. \%) }\end{array}$ & \begin{tabular}{|c|}
$\mathrm{Ag}$ \\
$(\mathrm{ppm})$
\end{tabular} & $\begin{array}{c}\text { As } \\
(\mathrm{ppm})\end{array}$ & \begin{tabular}{|c|}
$\mathrm{Ba}$ \\
$(\mathrm{ppm})$ \\
\end{tabular} & $\begin{array}{c}\mathrm{Be} \\
(\mathrm{ppm})\end{array}$ & $\begin{array}{c}\mathrm{Bi} \\
(\mathrm{ppm})\end{array}$ & $\begin{array}{c}\mathrm{Cd} \\
(\mathrm{ppm})\end{array}$ & $\begin{array}{c}\mathrm{Ce} \\
(\mathrm{ppm})\end{array}$ & $\begin{array}{c}\text { Co } \\
(\mathrm{ppm})\end{array}$ & \begin{tabular}{|c|}
$\mathrm{Cr}$ \\
$(\mathrm{ppm})$
\end{tabular} & $\begin{array}{c}\begin{array}{c}\mathrm{CS}^{\star} \\
(\mathrm{ppm})\end{array} \\
\end{array}$ & \begin{tabular}{|c|}
$\mathrm{Cu}$ \\
$(\mathrm{ppm})$ \\
\end{tabular} & \begin{tabular}{|c|} 
Ga \\
(ppm) \\
\end{tabular} & \begin{tabular}{|c|}
$\mathrm{Hg}$ \\
$(\mathrm{ppm})$
\end{tabular} \mid & \begin{tabular}{|c|} 
In \\
$(\mathrm{ppm})$
\end{tabular} & $\begin{array}{c}\text { La } \\
\text { (ppm) }\end{array}$ \\
\hline SP-04-1-1 O & 2004 & 3.49490 & -92.88660 & unburned & 69.35 & 5.72 & 2.65 & 1.71 & 1.69 & 0.56 & 2.01 & 0.07 & 0.17 & $<1$ & 3 & 820 & \begin{tabular}{l|}
1.2 \\
\end{tabular} & 0.26 & 0.7 & 21.9 & 7.5 & \begin{tabular}{l|}
22 \\
\end{tabular} & \begin{tabular}{|l|}
3.07 \\
\end{tabular} & \begin{tabular}{|l|}
13.9 \\
\end{tabular} & \begin{tabular}{|l|}
14.3 \\
\end{tabular} & \begin{tabular}{|l|}
0.05 \\
\end{tabular} & \begin{tabular}{|l|} 
\\
\end{tabular} & 10.9 \\
\hline SP-04-1-2 O & 2004 & .49495 & -92.88618 & low/medium & 7.20 & 4.80 & 6.40 & 1.37 & 2.29 & 1.08 & 1.70 & 0.30 & 0.15 & $<1$ & 2 & 1,440 & 1.0 & 0.26 & 3.0 & 19.4 & 8.2 & 17 & 4.20 & 31.7 & 12.0 & 0.07 & 0.02 & 10.1 \\
\hline SP-04-2-1 O & 2004 & 48.49599 & -92.88988 & unburned & 1.65 & 4.81 & 7.84 & 2.17 & 2.18 & 0.89 & 1.16 & 0.67 & 0.19 & $<1$ & 8 & 2,220 & 1.3 & 0.68 & 4.3 & 39.1 & 21.0 & 35 & 9.57 & 37.6 & 12.3 & 0.22 & 0.06 & 19.7 \\
\hline SP-04-2-2 O & 2004 & 48.49617 & -92.88976 & low/medium & .90 & 5.47 & 4.58 & 2.24 & 1.67 & 0.63 & 1.46 & 0.31 & 0.21 & $<1$ & 6 & 2,020 & 1.2 & 0.70 & 4.3 & 35.9 & 11.6 & 28 & 8.97 & 29.2 & 14.3 & 0.23 & 0.06 & 17.4 \\
\hline P-04-3-2 O & 004 & .49950 & -92.88380 & low/medium & .95 & 6.08 & 3.38 & 2.58 & 65 & 60 & 1.39 & 0.19 & 26 & $<1$ & 6 & 1,260 & 1.6 & 0.55 & 2.1 & 45.1 & 19.9 & 57 & 6.85 & 34.1 & 16.5 & 0.05 & 0.05 & 24.5 \\
\hline SP-04-3-3 O & 2004 & .49950 & -92.88430 & unburned & 4.39 & 4.33 & 11.00 & 2.22 & 1.85 & 1.39 & 1.08 & 0.72 & 0.19 & $<1$ & 6 & 1,850 & 0.9 & 0.46 & 2.4 & 31.5 & 13.8 & 34 & 10.00 & 71.8 & 11.6 & 0.11 & 0.04 & 17.4 \\
\hline SP-04-4-1 O & 2004 & 48.49866 & -92.89069 & unburned & 2.65 & 4.72 & 6.91 & 3.05 & 2.06 & .90 & 0.86 & 0.95 & 0.20 & $<1$ & 10 & 1,810 & 1.3 & 0.73 & 3.6 & 43.1 & 20.5 & 34 & 7.96 & 56.2 & 12.5 & 0.32 & 0.07 & 22.9 \\
\hline SP-04-4-1 O d & 2004 & 48.49866 & -92.89069 & unburned & 4.30 & 6.15 & 2.43 & 2.6 & 1.49 & 0.59 & 1.58 & 0.20 & 0.25 & $<1$ & 6 & 755 & 1.5 & 0.5 & 1.8 & 29.2 & 14.3 & 40 & 5.61 & 26.3 & 16.5 & 0.16 & 0.05 & 16.4 \\
\hline P-04-5-1 O & 2004 & .50055 & $\mid-92.88+x-2$ & unbur & 4.84 & 5.18 & 4.46 & 1.41 & 1.94 & 50 & 1.91 & 0.20 & 0.14 & $<1$ & 2 & 1,000 & 1.1 & 0.21 & 1.1 & 17.4 & 5.6 & 16 & 3.55 & 15.0 & 12.8 & 0.12 & 0.02 & 8.7 \\
\hline SP-04-5-1 O dup. & 2004 & 48.50055 & -92.88857 & |unburned & 17 & 5.79 & 2.69 & 1.7 & 1.77 & 55 & 2.04 & 10 & 17 & $<1$ & 2 & 894 & 1.2 & 0.27 & 0.7 & 20.5 & 7.3 & 19 & 2.89 & 14.6 & 14.6 & .07 & 0.03 & 10.4 \\
\hline -04-5-2 O & 2004 & 48.50 & -92.8 & low/r & & 4.71 & $8 . \varepsilon$ & 1.98 & 75 & & 41 & 0.55 & 17 & $<1$ & 5 & 2,120 & 1.2 & 0.41 & 3.4 & 27.3 & 9.3 & 27 & 7.31 & 40.2 & 11.6 & 0.14 & 0.03 & 14.1 \\
\hline$-04-6-10$ & 2 & .50142 & -92.8 & unbur & & 3.87 & 10.30 & & & & & 0.10 & & $<1$ & 5 & 2,370 & 0.9 & 0.60 & 7. & 25. & 9.7 & 31 & 11.40 & 59. & 10.8 & 0.12 & .05 & 12.9 \\
\hline$-04-6-2$ O & 2 & 70 & -92.8 & & .84 & 4.66 & 6.20 & 45 & & & & 31 & & $<1$ & 10 & 2,150 & 1.3 & .76 & 2. & 37.2 & 10.8 & 29 & 6.91 & 45.0 & 13.5 & 08 & 0.07 & 19.9 \\
\hline -04-7-1 O & 2004 & 48.505 & & unburned & & 3.54 & 8.69 & 58 & 2.27 & & & 32 & 15 & $<1$ & 11 & $2,040 \mid$ & 1.3 & 0.67 & 3.3 & 40.5 & 13.7 & 26 & 11.40 & 64.8 & 8.9 & .27 & 0.06 & 20.8 \\
\hline -04- & 200 & 48 & -92. & low/n & 18 & 6.11 & 4.78 & & & & & 59 & 0 & $<1$ & 14 & 1,980 & 2.9 & 1.02 & 3.1 & 86.1 & 33.1 & 41 & 11.50 & 47.7 & 13.8 & 0.29 & 0.10 & 41.5 \\
\hline$-04-\varepsilon$ & 2 & .50166 & -92.8 & unbur & & 3.61 & 10.50 & & & & & 0.85 & 0.1 & $<1$ & 5 & 2,930 & 1.2 & 0.47 & 5. & 42. & 25.7 & 42 & 13.40 & 73. & 8.6 & 0.15 & 0.03 & 21.7 \\
\hline-04 & & & & & & 22 & & & & & & & & $<1$ & 6 & 1,670 & 1.9 & & 3. & 59.7 & 46.1 & 46 & 7.02 & 40.5 & 14.5 & .21 & .05 & 31.2 \\
\hline$-04-8$ & 2004 & 48 & & high & 43.60 & 6.34 & 62 & & 61 & & & 21 & & $<1$ & 6 & 835 & 1.5 & 0.45 & 1.8 & 29.1 & 14.3 & 40 & 5.72 & 27.7 & 6.0 & .16 & .05 & 6.3 \\
\hline-04 & 2 & 50 & & & 2 & 68 & & & & & & & & $<1$ & 6 & 2,480 & 1.1 & 0.45 & 6.0 & 27. & 11.1 & 31 & 6.39 & 56. & 12.5 & 0.11 & 0.0 & 13.8 \\
\hline $\mathrm{O}$ & & 48 & & & 41.6 & 83 & 4.30 & 22 & 61 & & & 21 & & $<1$ & 5 & $\mid 1,030$ & 1.3 & 0.46 & 1.5 & 28.3 & 8.4 & 25 & 4.97 & 24.8 & 14.8 & .12 & 0.05 & 14.7 \\
\hline & & & & & & .24 & & & & & & & & $<1$ & 3 & $\mid 1,200$ & 1.3 & 0.26 & 1.3 & 17 & 5.3 & 18 & 3.70 & 19 & 13.1 & 0.06 & $<0.02$ & 9.0 \\
\hline$-05-1-10 \mathrm{~d}$ & 2005 & 48.49490 & -92.8 & unbs & 2 & 5.34 & & & 1.85 & .65 & & 1 & & $<1$ & 2 & 1,030 & 1.3 & 0.19 & 1.4 & 16 & 6.6 & 21 & 2.79 & 17.7 & 13.4 & 0.11 & 0.0 & 7.7 \\
\hline SP-05- & 2 & .49495 & \begin{tabular}{|l|l}
-92.88618 \\
\end{tabular} & low/medium & 23.2 & 4.44 & 7.63 & 1.21 & 65 & & & .41 & 0.13 & $<1$ & 2 & 1,820 & 1.2 & 0.31 & 4.6 & 18.6 & 7.8 & 30 & 5.00 & 37.8 & 10.7 & 0.10 & 0.03 & 9.5 \\
\hline & & & & unburned & & 4.86 & 6.93 & & 00 & & & .51 & & $<1$ & 6 & 2,010 & 1.3 & 0.56 & 3.1 & 33.1 & 23.2 & 39 & 7.77 & 30.7 & 12.6 & 0.16 & 0.05 & 16.7 \\
\hline & & & & high & & 28 & & & & & & & & $<1$ & 2 & 675 & 1.4 & & 1.1 & 15.7 & 7.2 & 29 & 3.17 & 22.7 & 13.0 & 0.04 & $<0.02$ & 8.1 \\
\hline SP-05-3-2C & 200 & 3.49950 & -92.88380 & low/n & & 5.52 & & & 56 & & & 0.35 & 0.2 & $<1$ & 5 & 1,280 & 1.7 & 0.56 & 2.6 & 42.2 & 16.9 & 36 & 7.02 & 42. & 15.4 & 0.06 & 0.05 & 23.1 \\
\hline-30 & 2 & .49950 & -92.88430 & & & 4.97 & & & & & & & & $<1$ & 4 & 1,170 & 1.2 & 0.33 & 1.1 & 24.2 & 9.7 & 28 & 5.24 & 33.3 & 13.7 & 0.08 & 0.03 & 12.7 \\
\hline & 2005 & & & & & 4.16 & 10.80 & & 95 & 28 & & 09 & 18 & $<1$ & 7 & $\mid 1,970$ & 1.2 & 0.64 & 4.1 & 36.2 & 14.1 & 31 & 10.80 & 68.5 & 10.8 & .19 & 0.06 & 19.3 \\
\hline & & & & & & & & & & & & & & $<1$ & 7 & 1,080 & 1. & & 1.5 & 34 & 7.9 & 45 & 10.90 & 46 & 18.6 & .17 & 0.05 & 18.1 \\
\hline SI & 2 & .50055 & -92. & & & 69 & & & & & & & & $<1$ & 2 & 909 & 1.2 & & 1 & 15 & 6.5 & 25 & 2.69 & 14 & 13. & 0.08 & $<0.02$ & 7.4 \\
\hline SP-05-5-2 O & 2005 & 48.50060 & -92.88860 & low/medium & 22.10 & 4.73 & 10.30 & 2 & 1.74 & 80 & .37 & .57 & .16 & $<1$ & 5 & 2,290 & 1.3 & 0.38 & 3.2 & 26.8 & 9.1 & 28 & 7.88 & 42.3 & 11.7 & 0.12 & 0.04 & 13.7 \\
\hline & 2005 & 48.50060 & -92.88840 & high & 42.90 & 5.45 & 6.26 & 2.16 & 1.71 & 61 & 60 & 0.25 & 0.19 & $<1$ & 8 & 2,130 & 1.4 & 0.59 & 3.4 & 33.6 & 9.9 & 28 & 7.30 & 36.3 & 13.7 & 0.16 & 0.06 & 16.9 \\
\hline & & & & & 16. & 4.93 & & & & & & & & $<1$ & 5 & 2,180 & 1.3 & & 6.1 & 25.4 & 9.6 & 33 & 10.60 & 54.8 & 12.9 & .16 & .05 & 13.1 \\
\hline & & & & & & & & & & & & & & $<1$ & 6 & & 1.0 & & & 29.1 & 12.4 & 21 & \begin{tabular}{|l|}
9.98 \\
\end{tabular} & 95 & 0. & $2+$ & & 16.9 \\
\hline SP-05-7-2 O & 2005 & 48.50501 & $\begin{array}{l}-92.89789 \\
\end{array}$ & low/medium & 18.55 & 5.48 & 5.43 & 2.96 & 2.00 & 0.71 & 01 & 0.48 & 0.20 & $<1$ & 8 & 2,010 & 2.0 & 0.70 & 4.0 & 44.5 & 35.2 & 36 & 11.10 & 54.0 & 14.0 & 0.23 & 0.07 & 23.2 \\
\hline SP-05-8-1 O & 2005 & 48.50166 & -92.87214 & unburned & 13.65 & 4.26 & 8.0 & 2.2 & 1.74 & 1.15 & 0.89 & 0.73 & 0.16 & $<1$ & 6 & 2,350 & 1.4 & 0.56 & 5.5 & 32.9 & 29.1 & 31 & 11.10 & 54.4 & 10.8 & 0.20 & 0.05 & 17.4 \\
\hline & 2005 & & -92.87230 & low/medium & 17. & 5.25 & & & & & & 0.0 & 0.2 & $<1$ & 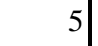 & 2,430 & 3.4 & 0.57 & 4.0 & 112.0 & 77.1 & 35 & 9.51 & 56.3 & 12.7 & 0.21 & 0.07 & 52.3 \\
\hline & & & & high & & 4.90 & & & & & & 0.00 & & $<1$ & 6 & 2,130 & 1.3 & 0.61 & 5.4 & 38.5 & 17.9 & 35 & 15.10 & 44.0 & 11.8 & 0.18 & 0.00 & 24.1 \\
\hline
\end{tabular}


Appendix 6-O Horizon Chemistry, Shoepack Lake, Voyageurs National Park, MN

\begin{tabular}{|c|c|c|c|c|c|c|c|c|c|c|c|c|c|c|c|c|c|c|c|c|c|c|c|c|c|c|c|c|c|}
\hline Field no. & \begin{tabular}{|c} 
Year \\
collected \\
\end{tabular} & Latitude & Longitude & Fire severity & $\begin{array}{c}\text { Ash } \\
\%\end{array}$ & $\begin{array}{c}\mathrm{Al} \\
\text { (wt. \%) }\end{array}$ & $\begin{array}{c}\mathrm{Ca} \\
\text { (wt. \%) }\end{array}$ & $\begin{array}{c}\mathrm{Fe} \\
\text { (wt. \%) }\end{array}$ & $\begin{array}{c}\mathrm{K} \\
\text { (wt. \%) }\end{array}$ & $\begin{array}{c}\mathrm{Mg} \\
\text { (wt. \%) }\end{array}$ & $\begin{array}{c}\mathrm{Na} \\
\text { (wt. \%) }\end{array}$ & $\begin{array}{c}\mathrm{S} \\
\text { (wt. \%) }\end{array}$ & $\begin{array}{c}\mathrm{Ti} \\
\text { (wt. \%) }\end{array}$ & $\begin{array}{c}\mathrm{Ag} \\
(\mathrm{ppm})\end{array}$ & $\begin{array}{c}\text { As } \\
\text { (ppm) }\end{array}$ & $\begin{array}{c}\mathrm{Ba} \\
(\mathrm{ppm})\end{array}$ & $\begin{array}{c}\mathrm{Be} \\
\text { (ppm) }\end{array}$ & $\begin{array}{c}\mathrm{Bi} \\
(\mathrm{ppm}\end{array}$ & $\begin{array}{c}\mathrm{Cd} \\
\text { (ppm) }\end{array}$ & $\begin{array}{c}\mathrm{Ce} \\
(\mathrm{ppm})\end{array}$ & $\begin{array}{c}\text { Co } \\
\text { (ppm) }\end{array}$ & \begin{tabular}{|c|}
$\mathrm{Cr}$ \\
$(\mathrm{ppm})$
\end{tabular} & $\begin{array}{c}\mathrm{Cs}^{\star} \\
\text { (ppm) }\end{array}$ & \begin{tabular}{|c}
$\mathrm{Cu}$ \\
$(\mathrm{ppm})$ \\
\end{tabular} & $\begin{array}{c}\mathrm{Ga} \\
(\mathrm{ppm} \\
\end{array}$ & & & $\begin{array}{c}\text { In } \\
(\mathrm{ppm})\end{array}$ & \begin{tabular}{|c|}
$\mathrm{La}$ \\
$(\mathrm{ppm})$ \\
\end{tabular} \\
\hline 05-9-2 O & 2005 & 48.50097 & -92.87247 & low/medium & 24.40 & 4.29 & 9.13 & 1.82 & 1.64 & 1.10 & 1.02 & 0.55 & 0.19 & $<1$ & & 2,760 & 1.5 & 0.4 & 5.6 & 36.6 & 42.5 & \begin{tabular}{|l|}
28 \\
\end{tabular} & 8.75 & \begin{tabular}{|l|l}
81.0 \\
\end{tabular} & 11. & & .09 & 0.05 & 196 \\
\hline $5-10-10$ & 2005 & 50210 & .88490 & & 28.35 & 5.33 & 04 & 2.26 & 1.64 & 0.95 & 1.44 & 0.30 & 0.23 & $<1$ & 6 & 1,860 & 1.3 & 0.5 & 4.5 & 30.5 & 10.4 & 36 & 6.36 & 37.2 & 14. & & 11 & .05 & 4.6 \\
\hline $5-10-2$ & 05 & & 9 & & & & & 1.83 & 1 & 57 & 1 & 17 & 0.20 & $<1$ & 5 & & 1.3 & 0.5 & 1.7 & 7.5 & & 27 & .56 & 20.2 & 4. & & & .05 & 4.0 \\
\hline $61-2$ & 2006 & & & & & & & 1.32 & & 0 & & & & $<1$ & 2 & & 1.2 & & 2.4 & 6. & & 26 & & 1.5 & 12 & & 05 & $<0.02$ & \\
\hline $062-10$ & 2006 & & & & .70 & 4.37 & 7.39 & 1.97 & 1.68 & 0.93 & 0.88 & 0.82 & 0.17 & $<1$ & & 1,840 & 1.2 & 0.4 & 3.3 & 27.5 & 20.2 & 33 & $<5$ & 42. & 11. & & 15 & 0.04 & \\
\hline $663-$ & 2006 & & & & 70 & 4.05 & 8.49 & 1.45 & 1.47 & 1.12 & 0.95 & 0.65 & 0.17 & $<1$ & 4 & 1,230 & 0.9 & 0.3 & 1.6 & 22.0 & 10.6 & 32 & $<5$ & 47.6 & 10. & & .07 & 0.03 & 1.8 \\
\hline $064-10$ & 2006 & & & & & 4.65 & & 1.60 & 1.60 & 0.95 & 1.09 & 0.49 & 0.20 & $<1$ & & & 1.1 & 0.4 & 2.0 & 34.5 & 21.8 & 41 & $<5$ & 35.6 & 3 & & .10 & .03 & 3.9 \\
\hline $065-10$ & 2006 & & & & & 5.49 & 5. & 2.01 & 1.77 & 0.70 & 1.5 & 0.49 & 0.15 & $<1$ & & 1,690 & 1.1 & 0.4 & 3. & 5.0 & 8.8 & ] & $<5$ & 33.2 & 2. & & .15 & .03 & 2.6 \\
\hline $065-10 d t$ & 06 & & & & 00 & 2.73 & 82 & 1.19 & 1.17 & 1.31 & 0.67 & 0.79 & 0.1 & $<1$ & 2 & 2,720 & 0.9 & 0.2 & 5.3 & 26.3 & 21.2 & 25 & $<5$ & 58.1 & 7.3 & & 1. & 0.02 & 0.3 \\
\hline 6 5-2 O & 06 & & & & 45.00 & 5.61 & 3.45 & 1.25 & 1.77 & 0.44 & 1.93 & 0.19 & 0.14 & 1 & & 773 & 1.1 & 0.1 & 0.9 & 14.9 & 5.6 & 18 & $<5$ & 12.1 & 12. & & .10 & $<0.02$ & \\
\hline 65 & & & & & & & & 65 & & & & & 0.20 & $<1$ & & & .4 & & 1.5 & & 11.7 & 9 & 6.00 & 0.1 & & & & 0.04 & \\
\hline 06 6- & 2006 & & & & 26.50 & 5.82 & 75 & 1.50 & 1.63 & 0.80 & 1.39 & 45 & 0.21 & 5 & & 1,280 & 1.2 & 0.5 & 2.7 & 8.5 & 12.1 & 34 & 6.00 & 37.6 & & & 13 & 0.04 & \\
\hline$-067-10$ & 2006 & 50540 & 2.89810 & & 9.00 & 5.15 & 63 & 3.02 & 2.45 & 1.21 & 0.76 & 1.35 & 0.14 & $<1$ & 8 & 1,690 & 2.3 & 0.5 & 4.7 & 79.4 & 28.5 & 29 & 11.00 & 76. & 10. & & .18 & 0.06 & 3.1 \\
\hline $068-$ & 2006 & & -92.87214 & & 14.30 & 4.18 & 9.19 & 1.83 & 1.53 & 1.45 & 0.87 & 0.96 & 0.14 & 4 & 3 & 2,680 & 1.1 & 0.3 & 6.1 & 26.1 & 24.3 & 30 & 9.00 & 62. & 10. & & .14 & 0.03 & 4.8 \\
\hline 69 & & & & & & 41 & 4 & 2.14 & 2.02 & 0.74 & 1.59 & 34 & 0.16 & $<1$ & & 1,790 & 1.1 & 0.3 & 3.0 & 5. & 8.8 & 29 & $<5$ & 4.8 & 3. & & .13 & 0.04 & 6. \\
\hline 06 10-1 O & 2006 & 0210 & & & 21.50 & 4.75 & 8.28 & 2.14 & 1.48 & 1.25 & 1.20 & 0.56 & 0.18 & $<1$ & 4 & 1,770 & 1.0 & 0.3 & & 3. & & & 6.00 & 3. & 2. & & .11 & 0.03 & \\
\hline
\end{tabular}




\begin{tabular}{|c|c|c|c|c|c|c|c|c|c|c|c|c|c|c|c|c|c|c|c|c|c|c|c|c|c|c|}
\hline Field no. & $\begin{array}{c}\text { Year } \\
\text { collected }\end{array}$ & Latitude & Longitude & Fire severity & $\begin{array}{c}\text { Ash } \\
\%\end{array}$ & $\begin{array}{c}\mathrm{Li} \\
(\mathrm{ppm})\end{array}$ & $\begin{array}{c}\mathrm{Mn} \\
(\mathrm{ppm}) \\
\end{array}$ & $\begin{array}{c}\text { Mo } \\
\text { (ppm) }\end{array}$ & $\begin{array}{c}\mathrm{Nb} \\
(\mathrm{ppm})\end{array}$ & \begin{tabular}{|c|}
$\mathrm{Ni}$ \\
$(\mathrm{ppm})$
\end{tabular} & $\begin{array}{c}\mathrm{P} \\
(\mathrm{ppm})\end{array}$ & $\begin{array}{c}\mathrm{Pb} \\
(\mathrm{ppm})\end{array}$ & $\begin{array}{c}\mathrm{Rb} \\
(\mathrm{ppm})\end{array}$ & $\begin{array}{c}\mathrm{Sb} \\
(\mathrm{ppm})\end{array}$ & $\begin{array}{c}\mathrm{Sc} \\
(\mathrm{ppm})\end{array}$ & $\begin{array}{c}\mathrm{Sn} \\
\text { (ppm) }\end{array}$ & \begin{tabular}{|c|}
$\mathrm{Sr}$ \\
$(\mathrm{ppm})$
\end{tabular} & $\begin{array}{c}\mathrm{Te} \\
(\mathrm{ppm})\end{array}$ & \begin{tabular}{|c|} 
Th \\
$(\mathrm{ppm})$
\end{tabular} & $\begin{array}{c}\mathrm{TI} \\
(\mathrm{ppm})\end{array}$ & $\begin{array}{c}U \\
(\mathrm{ppm})\end{array}$ & $\begin{array}{c}V \\
(\mathrm{ppm})\end{array}$ & $\begin{array}{c}W \\
(\mathrm{ppm})\end{array}$ & $\begin{array}{c}\mathrm{Y} \\
(\mathrm{ppm})\end{array}$ & $\begin{array}{c}\mathrm{Zn} \\
(\mathrm{ppm})\end{array}$ & $\begin{array}{c}\text { Se } \\
(\mathrm{ppm})\end{array}$ \\
\hline SP-04-1-1 O & 2004 & 48.49490 & -92.88660 & unburned & 69.35 & 16 & 1,620 & 0.87 & 5.0 & 21.2 & 1,390 & 41.0 & $\begin{array}{l}96.7 \\
\end{array}$ & 0.40 & 6.2 & 1.5 & 333 & $<0.1$ & \begin{tabular}{|l|}
4.3 \\
\end{tabular} & 0.5 & 1.1 & 47 & 0.5 & 7.4 & 96 & 0.3 \\
\hline SP-04-1-2 O & 2004 & 48.49495| & -92.88618 & low/medium & 27.20 & 13 & 4,730 & 1.45 & 4.3 & 22.4 & 4,250 & 46.2 & 123.0 & 0.56 & 5.1 & 2.2 & 514 & $<0.1$ & 3.0 & 0.3 & 0.9 & 39 & 0.5 & 6.3 & 341 & 0.3 \\
\hline P-04-2-1 O & 2004 & 48.49599 & -92.88988 & unburned & 21.65 & 16 & 12,661 & 2.26 & 6.5 & 32.3 & 6,990 & 133.0 & 156.0 & 1.86 & 7.1 & 3.1 & 632 & 0.1 & 5.3 & 1.1 & 1.6 & 64 & 0.9 & 11.0 & 376 & 0.6 \\
\hline$-04-2-2$ O & 04 & 49617 & -92.88976 & low/medium & .90 & 17 & 8,630 & 1.89 & 6.6 & 25.1 & 3,550 & 162.0 & 141.0 & 1.45 & 6.7 & 3.2 & 412 & 0.1 & 5.2 & 1.1 & 1.6 & 61 & 0.9 & 9.8 & 390 & 0.8 \\
\hline P-04-3-2 O & 2004 & 48.49950 & -92.88380 & low/medium & 50.95 & 19 & 2,170 & 1.70 & 8.0 & 42.0 & 3,200 & 77.9 & 90.9 & 1.12 & 7.8 & 2.7 & 312 & $<0.1$ & 5.4 & 0.6 & 11.0 & 78 & 1.0 & 12.1 & 323 & 0.9 \\
\hline SP-04-3-3 O & 2004 & 48.49950 & -92.88430 & unburned & 14.39 & 28 & 8,060 & 3.14 & 5.7 & 55.7 & 7,520 & 77.3 & 142.0 & 1.47 & 5.9 & 2.4 & 562 & $<0.1$ & 4.5 & 0.7 & 7.1 & 63 & 0.9 & 8.2 & 412 & 0.5 \\
\hline P-04-4-1 O & 2004 & 48.49866 & -92.89069 & unburned & 12.65 & 17 & 9,790 & 2.98 & 7.2 & 42.3 & 9,870 & 158.0 & 148.0 & 2.36 & 7.2 & 3.9 & 479 & 0.1 & 6.6 & 1.2 & 2.1 & 71 & 1.0 & 11.9 & 441 & 0.7 \\
\hline P-04-4-1 O dup. & 04 & 49866 & -92.89069 & unburned & .30 & 23 & 2,690 & 1.98 & 7.7 & 31.5 & 2,340 & 63 & 80 & 0.89 & 7.5 & 2.4 & 288 & $<0.1$ & 6 & 0.5 & 3.1 & 73 & 0.7 & 8.6 & 206 & 0.7 \\
\hline P-04-4-2 O & 2004 & 48.49850 & -92.89060 & low/medium & 11.11 & 15 & 5,420 & 3.33 & 7.3 & 40.4 & 11,274 & 166.0 & 140.0 & 3.65 & 7.3 & 5.3 & 368 & $<0.1$ & 5.8 & 1.2 & 6.5 & 69 & 1.2 & 12.7 & 602 & 0.5 \\
\hline SP-04-5-1 O & 2004 & 48.50055 & -92.88857 & unburned & 44.84 & 11 & 2,530 & 0.78 & 4.2 & 16.4 & 2,320 & 30.5 & 111.0 & 0.47 & 5.2 & 1.2 & 393 & $<0.1$ & 3.8 & 0.4 & 0.9 & 37 & 0.4 & 8.3 & 183 & 0.3 \\
\hline SP-04-5-1 O d & 2004 & 48.50055 & -92.88857 & unburned & 70.17 & 15 & 1,680 & 0.9 & 4.7 & 22.8 & 1,480 & 42 & 98.9 & 0.39 & 6 & 1.3 & 348 & $<0.1$ & 3.7 & 0.4 & 1 & 49 & 0.4 & 7 & 93 & 0.4 \\
\hline P-04-5-2 O & 2004 & .50060 & -92.88860 & low/medium & 29.60 & 15 & 7,620 & 1.65 & 5.3 & 30.1 & 6,240 & 60.2 & 133.0 & 1.03 & 5.6 & 1.9 & 609 & $<0.1$ & 4.8 & 1.0 & 1.3 & 49 & 0.6 & 9.0 & 556 & 0.5 \\
\hline SP-04-6-1 O & 2004 & 48.50142 & -92.88990 & unbu & 13.89 & 17 & 21,329 & 2.28 & 5.7 & 27.9 & 10,623 & 75.2 & 154.0 & 1.42 & 5.0 & 2.5 & 491 & $<0.1$ & 4.1 & 0.9 & 1.6 & 51 & 0.8 & 7.3 & 1,020 & 0.2 \\
\hline SP-04-6-2 O & 2004 & 48.50170 & -92.89010 & low/medium & 47.84 & 16 & 10,303 & 1.97 & 7.8 & 28.2 & 5,620 & 151.0 & 99.2 & 2.02 & 7.0 & 3.8 & 411 & $<0.1$ & 5.9 & 1.1 & 2.0 & 69 & 1.0 & 9.8 & 519 & 1.1 \\
\hline SP-04-7-1 O & 2004 & 48.50540 & -92.8 & unburned & 9.28 & 14 & 6,490 & 2.47 & 5.0 & 35.6 & 10,820 & 155.0 & 169.0 & 2.56 & 5.9 & 3.0 & 614 & $<0.1$ & 4.7 & 1.7 & 19.5 & 59 & 0.9 & 11.7 & 558 & 0.4 \\
\hline P-04-7-2 O & 2004 & 50501 & -92.89789 & low/medium & 8.80 & 21 & 6,060 & 3.11 & 8.4 & 59.0 & 8,240 & 206.0 & 129.0 & 3.2 & 9.9 & 5.3 & 391 & 0.1 & 7.5 & 1.7 & 12.6 & 80 & 1.3 & 21.3 & 383 & 0.8 \\
\hline SP-04-8-1 O & 04 & 50166 & & & 11.22 & 14 & 15,922 & 2.67 & 4.9 & 41.0 & 11,053 & 84.6 & 170.0 & 1.49 & 4.7 & 2.3 & 760 & $<0.1$ & 7.0 & 0.9 & 1.5 & 48 & 0.8 & 9.4 & 933 & 0.5 \\
\hline SP-04-8-2 O & 2004 & 48.50170 & -92.87230 & low/medium & 24.15 & 17 & 4,180 & 2.34 & 5.9 & 38.7 & 6,540 & 85.1 & 131.0 & 1.51 & 8.7 & 3.1 & 396 & $<0.1$ & 5.7 & 0.9 & 12.3 & 71 & 0.9 & 14.0 & 430 & 0.5 \\
\hline SP-04-8-3 O & 20 & 48.50 & -92. & high & 43.60 & 24 & 3,010 & 2.00 & 7.3 & 32.2 & 2,440 & 60.7 & 84.7 & 0.84 & 7.3 & 2.1 & 304 & $<0.1$ & 6.8 & 0.5 & 3.0 & 75 & 0.7 & 8.7 & 225 & 0.8 \\
\hline P-04-9-1 O & 2004 & 48.50080 & -92.87210 & unburn & 14.45 & 11 & 15,307 & 3.26 & 3.7 & 29.0 & 13,350 & 71.2 & 214.0 & 1.1 & 4.2 & 2.2 & 1140 & $<0.1$ & 3.0 & 0.6 & 1.1 & 36 & 0.6 & 7.3 & 1,290 & 0.5 \\
\hline SP-04-9-2 O & 2 & 48.50097 & $-92 . \varepsilon$ & low $/ \mathrm{m}$ & 34.45 & 15 & & & 6.7 & 32.5 & 6,200 & 112.0 & 170.0 & 1.4 & 7. & 3.5 & 598 & $<0.1$ & 5.8 & 0.8 & 1.6 & 58 & 0.8 & 9.7 & 539 & 0.8 \\
\hline SP-04-10-1 & & 48.50210 & & |unburned & 20.55 & 19 & 10,837 & 2.35 & .7 & 33.6 & 6,910 & 68.4 & 157.0 & 1 & 5.8 & 2.4 & 497 & $<0.1$ & 4.7 & 0.7 & 1.4 & 53 & 0.7 & 9.7 & 901 & 0.6 \\
\hline SP-04-1 & & & -92.8 & low/1 & 41.60 & 17 & 3,910 & & 8.0 & 27.1 & 2,250 & 71.1 & 103.0 & 0.9 & 6.5 & 2.1 & 360 & $<0.1$ & 5.9 & 0.6 & 1.5 & 58 & 0.8 & 8.5 & 134 & 0.6 \\
\hline SP-05- & 2005 & 48.49490 & -92.88660 & unburned & 38.40 & 9 & 2,330 & & 3.8 & 18.8 & 1,980 & 40.0 & 102.0 & 0.4 & 4.4 & 1.3 & 400 & $<0.1$ & 3.4 & 0.6 & 1.0 & 32 & 0.4 & 6.8 & 178 & 0.4 \\
\hline SP-05-1-1 O dup. & 2( & 48.49490 & -92.88660 & unburned & 22.80 & 15 & 3,060 & 0.82 & 3.6 & 22.6 & 3,380 & 24.2 & 85.9 & 0.31 & 4.6 & 1 & 392 & $<0.1$ & 3.6 & 0.4 & 0.9 & 36 & 0.4 & 5.6 & 310 & 0.3 \\
\hline SP-05-1-2 O & & & -92.88618 & low/medium & 23.25 & 10 & 6,750 & 1.65 & 3.6 & 25.1 & 4,870 & 48.5 & 95.1 & 0.71 & 4.1 & 1.5 & 580 & $<0.1$ & 2.9 & 0.5 & 1.0 & 33 & 0.4 & 5.9 & 521 & 0.4 \\
\hline SP- 05 & & & -92.8 & unbur & 20.50 & 14 & 11,720 & & 6.0 & 27.6 & 6,260 & 80.4 & 147.0 & & 6.7 & 2.7 & 535 & 0.1 & 5.0 & 1.2 & 1.5 & & 0.8 & 9.2 & 353 & 0.4 \\
\hline SP-05-3-1 O & 2005 & 48.49990 & -92.88320 & high & 25.05 & 12 & 3,790 & & 3.6 & 21.8 & 3,180 & 27.4 & 93.3 & 0.4 & 4.8 & 1.1 & 292 & $<0.1$ & 3.7 & 0.5 & 0.9 & 36 & 0.4 & 5.8 & 182 & 0.2 \\
\hline SP-05-3 & 2005 & 48.49950 & -92.88380 & low/medium & 19.85 & 17 & 2,880 & & 6.9 & 47.5 & 4,060 & 80.6 & 102.0 & 1.10 & 6.5 & 2.5 & 332 & $<0.1$ & 5.4 & 0.9 & 12.7 & 66 & 0.8 & 11.3 & 341 & 0.4 \\
\hline & & & & unburned & 22.10 & 19 & 3,270 & 2.05 & 6.1 & 394.0 & 3,490 & 40.7 & 99.3 & 0.59 & 5.5 & 1.9 & 403 & $<0.1$ & 4.3 & 0.6 & 6.5 & 54 & 0.7 & 7.5 & 220 & 0.4 \\
\hline & & & & & 6 & 5 & 12,760 & & 9 & 39.5 & 11,745 & 106.0 & 123.0 & & & 3.4 & 78 & $<0.1$ & & 1.1 & 2.2 & & 1.0 & 10.1 & 648 & 0.4 \\
\hline SP-05-4-2 O & 20 & 48.49850 & -92.89060 & low/1 & 23.1 & 26 & 4,210 & & 9.8 & 31.4 & 5,500 & 77.9 & 102.0 & & 8. & 3.4 & 266 & $<0.1$ & 6.3 & 0.9 & 7.4 & 79 & 1.3 & 10.1 & 390 & 0.6 \\
\hline SP-05-5-1 O & 2005 & 48.50055 & -92.88857 & unburned & 32.25 & 14 & 2,710 & 0.71 & 3.6 & 22.5 & 2,610 & 24.4 & 90.7 & 0.28 & 5.3 & 1.0 & 360 & $<0.1$ & 3.1 & 0.5 & 0.8 & 39 & 0.3 & 6.4 & 238 & $<0.2$ \\
\hline SP-05-5-2 O & & 48.50060 & -92.88860 & low/medium & 22.10 & 16 & 7,550 & 1.45 & 5.7 & 32.0 & 6,390 & 64.3 & 134.0 & 0.97 & 5.5 & 2.1 & 662 & $<0.1$ & 4.5 & 1.1 & 1.6 & 49 & 0.6 & 8.9 & 558 & 0.4 \\
\hline & & & & high & & 15 & & & 6.1 & 28.8 & 3,590 & 112.0 & 139.0 & & 6.4 & 2.8 & 543 & 0.1 & 6.3 & 0.9 & 1.5 & 56 & 0.7 & 10.0 & 413 & 0.6 \\
\hline & & 48 & -92.8 & & 16. & 19 & & & & 28.9 & 8,800 & 82.7 & & & & & 430 & $<0.1$ & & & 1.9 & & 0.8 & 9.0 & 30 & \\
\hline SP-05-7-1 O & 2005 & 48.50540 & -92.89810 & unburned & 7.89 & 10 & 11,409 & 2.28 & 3.6 & 34.9 & 14,987 & 71.1 & 188.0 & 1.61 & 3.9 & 2.0 & 951 & $<0.1$ & 3.6 & 1.5 & 11.7 & 39 & 0.7 & 9.0 & 911 & 0.4 \\
\hline SP-05-7-2 O & 2005 & 48.50501 & -92.89789 & low/medium & 18.55 & 21 & 9,940 & 2.48 & 7.3 & 43.6 & 6,720 & 127.0 & 135.0 & 2.05 & 7.3 & 3.8 & 374 & $<0.1$ & 6.3 & 1.7 & 8.5 & 68 & 1.1 & 12.4 & 523 & 0.6 \\
\hline & & & -92.87214 & & 5 & 15 & 14,593 & 2.63 & 5.5 & 35.9 & 8,820 & 122.0 & 160.0 & 1.5 & 5.9 & 3.0 & 594 & 0.1 & 4.6 & 0.8 & 1.9 & 56 & 0.8 & 9.7 & 796 & 0.7 \\
\hline -05-8-2 O & & & -92.87230 & low/medium & 17.80 & 18 & & 3.06 & 7.1 & 57.2 & 8,900 & 132.0 & 131.0 & 2.00 & 10.3 & 3.7 & 466 & $<0.1$ & 6.1 & 1.1 & 23.5 & 00 & 0.9 & 25.5 & 601 & 0.1 \\
\hline SP-05-8-3 O & 2005 & 48.50180 & -92.87250 & high & 23.35 & 17 & 9,670 & 2.54 & 6.0 & 31.4 & 5,240 & 105.0 & 111.0 & 1.52 & 6.1 & 2.9 & 576 & $<0.1$ & 5.4 & 0.7 & 2.3 & 63 & 0.8 & 8.4 & 629 & 0.7 \\
\hline SP-05-8-3 O du & 2005 & 48.50180 & -92.87250 & high & 10.45 & 14 & 13,906 & 2.86 & 5.5 & 39.2 & 13,207 & 95.2 & 143 & 2.02 & 6 & 3.3 & 717 & $<0.1$ & 5 & 1.2 & 2.1 & 57 & 0.9 & 10.4 & 713 & 0.4 \\
\hline SP-05-9-1 O & 2005 & 48.50080 & -92.87210 & unburned & 13.90 & 10 & 13,844 & 2.64 & 2.8 & 27.3 & 11,814 & 40.5 & 102.0 & 0.69 & 3.1 & 1.4 & 1140 & $<0.1$ & 2.6 & 0.5 & 1.1 & 29 & 0.4 & 6.1 & 1,310 & 0.3 \\
\hline
\end{tabular}


Appendix 6-O Horizon Chemistry, Shoepack Lake, Voyageurs National Park, MN

\begin{tabular}{|c|c|c|c|c|c|c|c|c|c|c|c|c|c|c|c|c|c|c|c|c|c|c|c|c|c|c|c|}
\hline Field no. & $\begin{array}{c}\text { Year } \\
\text { collected }\end{array}$ & titude & ongitude & Fire severity & $\begin{array}{c}\text { Ash } \\
\%\end{array}$ & $\begin{array}{c}\mathrm{Li} \\
(\mathrm{ppm})\end{array}$ & $\begin{array}{c}\mathrm{Mn} \\
(\mathrm{ppm})\end{array}$ & $\begin{array}{c}\text { Mo } \\
\text { (ppm) }\end{array}$ & $\begin{array}{c}\mathrm{Nb} \\
(\mathrm{ppm}) \\
\end{array}$ & $\begin{array}{c}\mathrm{Ni} \\
(\mathrm{ppm}) \\
\end{array}$ & $\begin{array}{c}\mathrm{P} \\
(\mathrm{ppm})\end{array}$ & $\begin{array}{c}\mathrm{Pb} \\
(\mathrm{ppm})\end{array}$ & $\begin{array}{c}\mathrm{Rb} \\
(\mathrm{ppm})\end{array}$ & $\begin{array}{c}\mathrm{Sb} \\
\text { (ppm) }\end{array}$ & $\begin{array}{c}\text { Sc } \\
\text { (ppm) }\end{array}$ & $\begin{array}{c}\begin{array}{c}\mathrm{Sn} \\
(\mathrm{ppm})\end{array} \\
\end{array}$ & $\begin{array}{c}\mathrm{Sr} \\
(\mathrm{ppm})\end{array}$ & $\begin{array}{c}\mathrm{Te} \\
(\mathrm{ppm}\end{array}$ & $\begin{array}{c}\text { Th } \\
(\mathrm{ppm} \\
\end{array}$ & $\begin{array}{r}\mathrm{Tl} \\
\text { (pp } \\
\end{array}$ & & & $\begin{array}{c}\mathrm{V} \\
(\mathrm{ppm})\end{array}$ & \begin{tabular}{|c|}
$\begin{array}{c}\text { W } \\
(\mathrm{ppm})\end{array}$ \\
\end{tabular} & $\begin{array}{c}\mathrm{Y} \\
\text { (ppm) }\end{array}$ & $\begin{array}{c}\begin{array}{c}\mathrm{Zn} \\
(\mathrm{ppm}\end{array} \\
\end{array}$ & $\begin{array}{c}\text { Se } \\
(\mathrm{ppm})\end{array}$ \\
\hline$-05-9-2$ O & 2005 & 50097 & 2.87247 & low/me & 24.40 & 15 & 9,810 & 1.68 & 6.2 & 36.8 & 7,150 & 77.9 & 139.0 & 1.10 & 6.2 & 2.6 & 777 & $<0$. & 5. & & & 2.3 & 56 & \begin{tabular}{|l|}
0.7 \\
\end{tabular} & 9.3 & 1,06 & 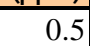 \\
\hline-05 & & & & & & & & & 4 & .6 & 650 & 70.3 & 7.0 & & & 2.5 & 39 & $<0$. & & & & 1.7 & & & & & 0.5 \\
\hline-05 & & & & & & 1 & & & 5.9 & 25.1 & 840 & 81.7 & 102.0 & 1.00 & & 2.6 & 30 & $<0$. & & & & 1.4 & & & & & \\
\hline P-06 & 26 & & & & 3270 & 14 & 3,250 & 1.80 & 4.1 & 22.1 & 3,800 & 24.0 & 83.6 & 0.24 & 4.9 & 1.0 & 425 & $<0$ & 2. & & & 0.8 & 37 & 0.6 & 5.4 & 31 & $<0.2$ \\
\hline-06 & & & & & 13.70 & 14 & 10,029 & 2.19 & 6.1 & 26.0 & 9,900 & 74.2 & 89.9 & 1.23 & 6.5 & 2.9 & $50 €$ & $<0$ & 4. & & & 1.3 & 48 & 0.7 & 9.2 & 39 & 0.2 \\
\hline-0 & & & & & & 14 & & & 5.2 & 33.9 & 6,680 & 40.3 & 97.9 & 0.61 & 5.3 & 2.0 & 472 & $<0$. & 3. & & & 5.5 & 44 & 0.8 & 6.7 & 31 & $<0.2$ \\
\hline P-06 & & & & & 9.3 & 15 & 1 & 2.1 & 6.7 & 19.3 & 4,460 & 56.4 & 96.0 & 0.85 & 6. & 2.5 & 408 & $<0$. & & & & 2.4 & & U. & & 28 & $<0.2$ \\
\hline P-06 & & & & & 2.30 & 15 & & 1.54 & 4.7 & 27.7 & 5,670 & 62.0 & 111.0 & 0.81 & 5.8 & 2.2 & 453 & $<0$. & 3. & & & 1.2 & +2 & 0.6 & 7.9 & 41 & 0.3 \\
\hline-06 & & & & & 3.00 & 9 & 10,755 & 2.24 & 2.9 & 19.9 & 7,920 & 42.9 & 71.8 & 0.57 & 4.3 & 1.6 & 785 & $<0$. & 2. & & & 0.8 & 27 & 2.7 & 5.9 & 75 & 0.3 \\
\hline-0 & & & & & & 10 & & & 4.1 & 3.7 & 1,840 & 25.5 & 91.5 & 0.21 & .9 & 1.2 & 318 & $<0$. & & & & 0.7 & 1 & 0.8 & & 2 & $<0.2$ \\
\hline P-06 & & & & hi & 367 & 20 & & & 6.2 & 27.7 & 2,540 & 67.6 & 104.0 & 0.95 & 74 & 2.4 & 39 & $<0$. & & & & 1.2 & & & & 22 & \\
\hline P-06 & & & & & 26.50 & 17 & 10,307 & 1.88 & 7.1 & 19.5 & 5,230 & 88.9 & 97.0 & 1.20 & 5.4 & 5.3 & 378 & $<0$. & 4. & & & 1.5 & & .9 & 8.2 & 36 & 0.3 \\
\hline -06? ? & 2006 & & & & 9.00 & 14 & 4,660 & 2.92 & 5.2 & 42.5 & 14,356 & 121.0 & 168.0 & 2.02 & 8.0 & 3.5 & 523 & $<0$. & 4. & & & 76.6 & 54 & 0.9 & 25.0 & 39 & 0.3 \\
\hline-06 & & & & & & 14 & $14,4 \mathrm{c}$ & 2.3 & 4.6 & 30.4 & 10,214 & 55.8 & 109.0 & 0.96 & 4.7 & 2.2 & 72 & $<0$. & & & & 1.4 & 41 & .1 & & 76 & J. \\
\hline$-0 t$ & & & & & 22.00 & 15 & 6,1 & 1.4 & 5.1 & 23.8 & 6,230 & 60.2 & 118.0 & 0.97 & 0.1 & 2.1 & 49 & $<0$. & & & & 1.2 & & 1.2 & 8.1 & & 0.2 \\
\hline P-06 10-1 O & 006 & .50210 & 92.88490 & unburned & 1.50 & 19 & 7,790 & 3.14 & 5.9 & 28.3 & 8,140 & 48.9 & 110.0 & 0.70 & 6.3 & 1.9 & 435 & $<0$. & 3. & & & 1.1 & 49 & 0.7 & 8.0 & 70 & 0.3 \\
\hline
\end{tabular}




\section{Click back to Contents}

Appendix 7-C Horizon Chemistry, Shoepack Lake, Voyageurs National Park, MN

\begin{tabular}{|c|c|c|c|c|c|c|c|c|c|c|c|c|c|c|c|c|c|c|c|c|c|c|c|c|c|c|c|c|c|}
\hline Field no. & $\begin{array}{c}\text { Year } \\
\text { collected }\end{array}$ & Latitude & Longitude & Fire severity & \begin{tabular}{|c|} 
Depth \\
$(\mathrm{cm})$
\end{tabular} & $\begin{array}{c}\begin{array}{c}\mathrm{Al} \\
\text { (wt. \%) }\end{array} \\
\end{array}$ & $\begin{array}{c}\mathrm{Ca} \\
\text { (wt. \%) }\end{array}$ & \begin{tabular}{|c|}
$\mathrm{Fe}$ \\
(wt. \%)
\end{tabular} & \begin{tabular}{|c|}
$\mathrm{K}$ \\
(wt. \%)
\end{tabular} & \begin{tabular}{|c|}
$\mathrm{Mg}$ \\
(wt. \%)
\end{tabular} & \begin{tabular}{|c|}
$\mathrm{Na}$ \\
(wt. \%)
\end{tabular} & \begin{tabular}{|c|c} 
S \\
(wt. \%)
\end{tabular} & $\begin{array}{c}\mathrm{Ti} \\
\text { (wt. \%) }\end{array}$ & $\begin{array}{c}\mathrm{Ag} \\
(\mathrm{ppm})\end{array}$ & \begin{tabular}{|c|} 
As \\
(ppm)
\end{tabular} & $\begin{array}{c}\mathrm{Ba} \\
\text { (ppm) }\end{array}$ & \begin{tabular}{|c|}
$\mathrm{Be}$ \\
(ppm)
\end{tabular} & $\begin{array}{c}\mathrm{Bi} \\
(\mathrm{ppm})\end{array}$ & \begin{tabular}{|c|}
$\mathrm{Cd}$ \\
$(\mathrm{ppm})$
\end{tabular} & \begin{tabular}{|c|}
$\mathrm{Ce}$ \\
$(\mathrm{ppm})$
\end{tabular} & \begin{tabular}{c|} 
Co \\
$(\mathrm{ppm})$
\end{tabular} & $\begin{array}{c}\mathrm{Cr} \\
(\mathrm{ppm})\end{array}$ & \begin{tabular}{|c|} 
Cs \\
(ppm)
\end{tabular} & \begin{tabular}{|c|}
$\begin{array}{c}\mathrm{Cu} \\
(\mathrm{ppm})\end{array}$ \\
\end{tabular} & \begin{tabular}{|c|}
$\mathrm{Ga}$ \\
(ppm)
\end{tabular} & \begin{tabular}{|c|}
$\mathrm{Hg}$ \\
$(\mathrm{ppm})$
\end{tabular} & \begin{tabular}{|c|} 
In \\
$(\mathrm{ppm})$
\end{tabular} & \begin{tabular}{|c|} 
La \\
$($ ppm $)$
\end{tabular} & $\begin{array}{c}\mathrm{Li} \\
\text { (ppm) }\end{array}$ \\
\hline SP-1-1 C & 2004 & 48.49490 & -92.88660 & unburned & 61 & 6.61 & 1.36 & 1.71 & 1.74 & $\begin{array}{l}0.50 \\
\end{array}$ & 2.28 & $<0.01$ & 0.14 & $<1$ & 2 & 423 & 1.5 & 0.12 & $<0.1$ & \begin{tabular}{|l|}
17.7 \\
\end{tabular} & 6.5 & 34 & 1.80 & \begin{tabular}{|l|}
10.5 \\
\end{tabular} & 14.0 & $<0.02$ & $<0.02$ & 9.6 & 15 \\
\hline SP-2-2 C & 2004 & & & low/1 & 46 & 6.90 & 1.40 & 1.94 & 1.87 & 0.64 & 2.41 & $<0.01$ & 0.14 & $<1$ & 2 & 447 & 1.5 & 0.16 & $<0.1$ & 21.8 & 7.9 & 41 & 1.56 & 12.0 & 14.5 & $<0.02$ & $<0.02$ & 11.2 & 18 \\
\hline SP-2-2 C dup & 2004 & 48.49617 & $-92.88 c$ & low/me & 46 & $6.7 \mathrm{c}$ & 1.39 & 1.94 & 1.7 & 0.6 & 2.36 & $<0.01$ & 0.14 & $<1$ & 2 & 436 & 1.6 & 0.16 & $<0.1$ & 22.4 & 7.8 & 35 & 1.51 & 11.2 & 14.2 & $<0.02$ & 0.02 & 11.4 & 17 \\
\hline SP-4-3 C & 2004 & 48.49830 & -92.89090 & high & 40 & 6.68 & 1.28 & 1.99 & 1.68 & 0.51 & 2.11 & 0.01 & 0.16 & $<1$ & 2 & 429 & 1.3 & 0.13 & $<0.1$ & 19.0 & 8.7 & 37 & 2.03 & 8.8 & 14.3 & 0.03 & 0.02 & 9.4 & 16 \\
\hline SP-5-3 C & 2004 & 48.50060 & -92.88840 & high & 50 & 6.87 & 1.43 & 2.05 & 1.79 & 0.58 & 2.35 & 0.01 & 0.17 & $<1$ & 2 & 434 & 1.5 & 0.11 & $<0.1$ & 14.4 & 7.2 & 45 & 1.88 & 7.7 & 14.4 & $<0.02$ & 0.02 & 7.9 & 19 \\
\hline & 2004 & 48.50480 & -92.89560 & high & 45 & 6.37 & 1.32 & 1.58 & 1.68 & 0.42 & 2.32 & 0.01 & 0.15 & $<1$ & 1 & 428 & 1.4 & 0.13 & $<0.1$ & 15.8 & 5.8 & 53 & 1.65 & 5.4 & 14.0 & $<0.02$ & $<0.02$ & 8.4 & 16 \\
\hline & 2004 & & & & 61 & 73 & 1.36 & 3.17 & 1.29 & 0.88 & 813 & 0.02 & 0.22 & & & 346 & 1. & 0.2 & & & & & & 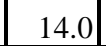 & & & & & \\
\hline
\end{tabular}


Appendix 7-C Horizon Chemistry, Shoepack Lake, Voyageurs National Park, MN

\begin{tabular}{|c|c|c|c|c|c|c|c|c|c|c|c|c|c|c|c|c|c|c|c|c|c|c|c|c|c|c|}
\hline Field no. & $\begin{array}{c}\text { Year } \\
\text { collected }\end{array}$ & Latitude & Longitude & Fire severity & \begin{tabular}{|c|} 
Depth \\
(cm)
\end{tabular} & $\begin{array}{c}\mathrm{Mn} \\
(\mathrm{ppm}) \\
\end{array}$ & $\begin{array}{c}\text { Mo } \\
\text { (ppm) } \\
\end{array}$ & $\begin{array}{c}\mathrm{Nb} \\
(\mathrm{ppm})\end{array}$ & $\begin{array}{c}\mathrm{Ni} \\
(\mathrm{ppm})\end{array}$ & $\begin{array}{c}\mathbf{P} \\
\text { (ppm) }\end{array}$ & $\begin{array}{c}\mathrm{Pb} \\
(\mathrm{ppm})\end{array}$ & $\begin{array}{c}\mathbf{R b} \\
(\mathrm{ppm})\end{array}$ & $\begin{array}{c}\mathrm{Sb} \\
(\mathrm{ppm})\end{array}$ & \begin{tabular}{|c|} 
Sc \\
(ppm)
\end{tabular} & $\begin{array}{c}\text { Sn } \\
\text { (ppm) }\end{array}$ & $\begin{array}{c}\mathrm{Sr} \\
(\mathrm{ppm})\end{array}$ & \begin{tabular}{|c|}
$\mathrm{Te}$ \\
$(\mathrm{ppm})$ \\
\end{tabular} & \begin{tabular}{|l|} 
Th \\
(ppm)
\end{tabular} & $\begin{array}{c}\mathrm{TI} \\
(\mathrm{ppm})\end{array}$ & $\begin{array}{c} \\
(\mathrm{ppm}) \\
\end{array}$ & $\begin{array}{c}\mathrm{V} \\
(\mathrm{ppm})\end{array}$ & \begin{tabular}{|c|}
$\begin{array}{c}\text { W } \\
\text { (ppm) }\end{array}$ \\
\end{tabular} & $\begin{array}{c}\mathrm{Y} \\
\text { (ppm) }\end{array}$ & \begin{tabular}{|c|}
$\mathrm{Zn}$ \\
$(\mathrm{ppm})$
\end{tabular} & $\begin{array}{c}\mathrm{Se} \\
(\mathrm{ppm})\end{array}$ & \begin{tabular}{|l} 
Total C \\
(wt. \%) \\
\end{tabular} \\
\hline $1-1 \mathrm{C}$ & 2004 & 48.49490 & -92.8866 & unburned & 61 & 241 & 0.21 & 3.7 & 19.5 & 260 & 15.5 & \begin{tabular}{r|}
68.5 \\
\end{tabular} & 0.10 & 5.6 & 0.9 & 275 & $<0.1$ & 3.1 & 0.4 & 1.0 & 44 & 0.3 & 5.4 & \begin{tabular}{|l|}
30 \\
\end{tabular} & $<0.2$ & 0.41 \\
\hline$-2-2$ & & & & & 46 & 303 & 0.26 & 3.9 & 24.4 & 360 & 16.5 & 82.3 & 0.09 & 6.1 & 2.2 & 271 & $<0.1$ & 3.8 & 0.5 & 1.1 & 50 & 0.4 & 5.7 & 29 & $<0.2$ & 0.45 \\
\hline P-2-2 C du & 2004 & 48 & & low & 46 & 301 & 0.2 & 4.0 & 23.0 & 340 & 16.3 & 75.4 & 0.09 & 6.1 & 1.9 & 269 & $<0.1$ & 4.1 & 0.4 & 1.2 & 51 & 0.3 & 5.6 & 30 & $<0.2$ & 0.42 \\
\hline SP-4-3 C & 2004 & 48.49830 & -92.89090 & high & 40 & 273 & 0.48 & 4.4 & 26.3 & 340 & 16.1 & 73.3 & 0.15 & 5.7 & 0.8 & 253 & $<0.1$ & 4.4 & 0.4 & 1.4 & 52 & 0.5 & 5.6 & 42 & 0.4 & 0.92 \\
\hline P-5-3 C & 2004 & 50060 & -92.88840 & high & 50 & 325 & 0.46 & 4.4 & 18.9 & 180 & 16.4 & 76.9 & 0.16 & 5.8 & 2.2 & 278 & $<0.1$ & 3.0 & 0.4 & 0.9 & 51 & 0.3 & 5.0 & 35 & $<0.2$ & 1.08 \\
\hline & & & & high & 45 & 276 & 0.52 & 4.3 & 17.1 & 360 & 16.1 & 82.1 & 0.15 & 5.3 & 0.8 & 265 & $<0.1$ & 3.3 & 0.4 & 0.9 & 40 & 0.3 & 5.8 & 28 & $<0.2$ & 1.18 \\
\hline P-8-3 C & 2004 & 48.50180 & -92.87250 & high & 61 & 344 & 0.76 & 5.7 & 28.9 & 1,010 & 16.9 & 64.7 & 0.20 & 7.8 & 2.2 & 275 & $<0.1$ & 5.1 & 0.4 & 2.7 & 78 & 0.7 & 6.1 & 54 & 0.4 & 1.65 \\
\hline
\end{tabular}


Click back to Contents

Appendix 8-Water Quality Data, Selected Lakes at Voyageurs National Park, MN

\begin{tabular}{|c|c|c|c|c|c|c|c|c|c|c|c|c|c|c|c|c|c|c|c|c|c|}
\hline \multirow[b]{2}{*}{ Station no. } & \multirow[b]{2}{*}{ Lake } & \multirow{2}{*}{\multicolumn{3}{|c|}{ Collection Date }} & \multirow[b]{2}{*}{$\begin{array}{c}\text { Sample } \\
\text { no. }\end{array}$} & \multirow{2}{*}{$\begin{array}{c}\text { Sampling } \\
\text { depth } \\
\text { (m) }\end{array}$} & \multirow{2}{*}{$\begin{array}{c}\text { Secchi disc } \\
\text { transparency } \\
\text { (m) }\end{array}$} & \multirow{2}{*}{$\begin{array}{c}\text { Dissolved } \\
\text { oxygen } \\
\text { (mg/L) }\end{array}$} & \multirow[b]{2}{*}{ pH [field] } & \multirow[b]{2}{*}{ pH [lab] } & \multicolumn{2}{|c|}{$\begin{array}{c}\text { Specific } \\
\text { conductance }\end{array}$} & \multirow{2}{*}{\begin{tabular}{|c} 
Air \\
temperature \\
$\left({ }^{\circ} \mathrm{C}\right)$
\end{tabular}} & \multirow{2}{*}{$\begin{array}{c}\text { Water } \\
\text { temperature } \\
\left({ }^{\circ} \mathrm{C}\right)\end{array}$} & \multirow[b]{2}{*}{$\begin{array}{c}\text { Calcium } \\
(\mathrm{mg} / \mathrm{L})\end{array}$} & \multirow[b]{2}{*}{$\begin{array}{c}\text { Magnesium } \\
(\mathrm{mg} / \mathrm{L})\end{array}$} & \multirow[b]{2}{*}{$\begin{array}{c}\text { Potassium } \\
\text { (mg/L) }\end{array}$} & \multirow[b]{2}{*}{\begin{tabular}{|c|} 
\\
Sodium \\
$(\mathrm{mg} / \mathrm{L})$
\end{tabular}} & \multirow[b]{2}{*}{$\begin{array}{c}\text { Alkalinity } \\
\text { (mg/L) }\end{array}$} & \multirow[b]{2}{*}{$\begin{array}{c}\text { Bicarbonate } \\
\text { (mg/L) }\end{array}$} & \multirow[b]{2}{*}{$\begin{array}{c}\text { Carbonate } \\
(\mathrm{mg} / \mathrm{L})\end{array}$} \\
\hline & & & & & & & & & & & \begin{tabular}{|c} 
Field \\
$(\mu \mathrm{S} / \mathrm{cm})$
\end{tabular} & $\begin{array}{c}\text { Lab } \\
(\mu \mathrm{S} / \mathrm{cm})\end{array}$ & & & & & & & & & \\
\hline 483129092462001 & Peary & 5 & 8 & 2003 & 300338 & 0.05 & & & & & & & & & & & & & & & \\
\hline 483109092422601 & Ryan & 5 & 8 & 2003 & 300339 & 0.05 & & & & & & & & & & & & & & & \\
\hline 483059092474501 & Brown & 5 & 9 & 2003 & 300340 & 0.05 & & & & & & & & & & & & & & & \\
\hline 483059092474501 & Brown & 5 & 19 & 2004 & 400181 & 0.05 & 3.0 & 11.4 & 6.9 & 7.0 & 22 & 26 & & 12.9 & 2.10 & 0.852 & 0.60 & 1.08 & 6 & 8 & 0 \\
\hline 483129092462001 & Peary & 5 & 19 & 2004 & 400171 & 0.05 & 2.3 & 11.8 & & 7.1 & 26 & 30 & 18.5 & 13.1 & 2.48 & 1.060 & 0.71 & 1.14 & 10 & 8 & 0 \\
\hline 483109092422601 & Ryan & 5 & 19 & 2004 & 400173 & 0.05 & 3.5 & 11.1 & 6.8 & 7.2 & 27 & 31 & 16.5 & 13.4 & 2.65 & 0.953 & 0.56 & 1.10 & 6 & 7 & 0 \\
\hline 483059092474501 & Brown & 8 & 5 & 2004 & 400358 & 0.05 & 3.0 & 8.3 & 7.4 & 6.6 & 26 & 26 & 22.5 & 24.2 & 2.10 & 0.884 & 0.65 & 1.10 & 5 & 6 & \\
\hline 483129092462001 & Peary & 8 & 5 & 2004 & $\mid 400357$ & 0.05 & 2.5 & 8.5 & 7.3 & 6.4 & 32 & 31 & 24.0 & 26.7 & 2.65 & 1.160 & 0.61 & 1.17 & 10 & 12 & 0 \\
\hline 483109092422601 & Ryan & 8 & 5 & 2004 & 400356 & 0.05 & 2.5 & 8.4 & 7.0 & 6.5 & 30 & 29 & & 22.8 & 2.49 & 0.995 & 0.53 & 1.12 & 6 & 7 & 0 \\
\hline 482951092531601 & Shoepack & 8 & 25 & 2004 & 400624 & 0.05 & & & & & & & & & & & & & & & \\
\hline 482951092531601 & Shoepack & 9 & 22 & 2004 & 400613 & 0.05 & 1.5 & 8.6 & 6.4 & 7.1 & 29 & 35 & 13.5 & 16.8 & 2.68 & 1.150 & 0.85 & 1.23 & 4 & 5 & 0 \\
\hline 483059092474501 & Brown & 9 & 23 & 2004 & 400588 & 0.05 & 2.5 & 9.2 & 7.0 & 7.0 & 23 & 27 & 21.5 & 17.2 & 2.08 & 0.853 & 0.62 & 1.06 & 6 & 8 & 0 \\
\hline 483129092462001 & Peary & 9 & 23 & 2004 & 400607 & 0.05 & 2.7 & 8.9 & 6.9 & 6.9 & 28 & 32 & 18.0 & 16.9 & 2.56 & 1.150 & 0.57 & 1.17 & 10 & 12 & 0 \\
\hline 483109092422601 & Ryan & 9 & 23 & 2004 & 400594 & 0.05 & 2.0 & 8.8 & 6.8 & 7.0 & 27 & 32 & 15.0 & 16.8 & 2.48 & 0.954 & 0.55 & 1.12 & 6 & 7 & 0 \\
\hline 482951092531601 & Shoepack & 5 & 12 & 2005 & 500325 & 0.05 & & 9.9 & 6.2 & 6.7 & 26 & 31 & & 11.7 & 2.40 & 1.040 & 0.71 & 1.06 & 4 & 4 & 0 \\
\hline 482951092531601 & Shoepack & 5 & 12 & 2005 & 500611 & 0.05 & & & & & & & & & & & & & & & \\
\hline 483059092474501 & Brown & 5 & 13 & 2005 & 500322 & 0.05 & 2.4 & 10.1 & 6.6 & 6.8 & 23 & 25 & & 10.9 & 1.96 & 0.877 & 0.60 & 0.98 & 6 & 7 & 0 \\
\hline 483129092462001 & Peary & 5 & 13 & 2005 & 500323 & 0.05 & 2.0 & 10.4 & 6.8 & 7.0 & 27 & 28 & & 11.1 & 2.50 & 1.110 & 0.64 & 1.07 & 8 & 10 & 0 \\
\hline 483109092422601 & Ryan & 5 & 13 & 2005 & 500324 & 0.05 & 3.2 & 10.1 & 6.5 & 6.8 & 27 & 28 & & 11.1 & 2.46 & 0.985 & 0.48 & 1.01 & 4 & 5 & 0 \\
\hline 483059092474501 & Brown & 7 & 13 & 2005 & 500388 & 0.05 & 2.4 & 7.2 & 7.1 & & 25 & & & 29.0 & & & & & & & \\
\hline 483129092462001 & Peary & 7 & 13 & 2005 & 500389 & 0.05 & 2.1 & 7.2 & 7.3 & & 29 & & & 28.7 & & & & & & & \\
\hline 482951092531601 & Shoepack & 7 & 14 & 2005 & 500390 & 0.05 & 1.2 & 8.8 & 7.2 & & 30 & & & 27.3 & & & & & & & \\
\hline 483109092422601 & Ryan & 7 & 15 & 2005 & 500612 & 0.05 & & & & & & & & & & & & & & & \\
\hline 483059092474501 & Brown & 9 & 14 & 2005 & 500528 & 0.05 & 2.0 & 8.5 & 6.9 & & 27 & & 15.0 & 19.1 & & & & & & & \\
\hline 483129092462001 & Peary & 9 & 14 & 2005 & 500529 & 0.05 & 2.5 & 8.2 & 6.9 & & 29 & & 14.0 & 19.2 & & & & & & & \\
\hline 483109092422601 & Ryan & 9 & 14 & 2005 & 500527 & 0.05 & 1.5 & 8.3 & 6.5 & & 31 & & 13.5 & 19.2 & & & & & & & \\
\hline 483059092474501 & Brown & 5 & 16 & 2006 & 600190 & 0.05 & 3.0 & 10.1 & 6.5 & & 19 & & & 13.8 & & & & & & & \\
\hline 483129092462001 & Peary & 5 & 16 & 2006 & 600189 & 0.05 & 2.6 & 10.1 & 6.8 & & 22 & & & 14.0 & & & & & & & \\
\hline 483109092422601 & Ryan & 5 & 16 & 2006 & 600192 & 0.05 & 3.5 & 8.9 & 6.5 & & 24 & & & 15.4 & & & & & & & \\
\hline 482951092531601 & Shoepack & 5 & 17 & 2006 & 600191 & 0.05 & 1.2 & 9.9 & 6.0 & & 20 & & & 13.9 & & & & & & & \\
\hline 482951092531601 & Shoepack & 7 & 27 & 2006 & 600710 & 0.05 & & & & & & & & & & & & & & & \\
\hline 483059092474501 & Brown & 7 & 28 & 2006 & 600712 & 0.05 & & & & & & & & & & & & & & & \\
\hline 483129092462001 & Peary & 7 & 28 & 2006 & 600711 & 0.05 & & & & & & & & & & & & & & & \\
\hline 483109092422601 & Ryan & 7 & 28 & 2006 & 600708 & 0.05 & & & & & & & & & & & & & & & \\
\hline 482805092484101 & Agnes & 8 & 2 & 2006 & 600709 & 0.05 & & & & & & & & & & & & & & & \\
\hline 482951092531601 & Shoepack & 9 & 15 & 2006 & 500526 & 0.05 & 1.5 & 7.9 & 6.1 & & 27 & & 18.0 & 18.5 & & & & & & & \\
\hline
\end{tabular}




\begin{tabular}{|c|c|c|c|c|c|c|c|c|c|c|c|c|c|c|c|c|c|c|c|c|c|c|}
\hline \multirow[b]{2}{*}{ Station no. } & \multirow[b]{2}{*}{ Lake } & \multirow{2}{*}{\multicolumn{3}{|c|}{ Collection Date }} & \multirow[b]{2}{*}{$\begin{array}{c}\text { Sample } \\
\text { no. }\end{array}$} & \multirow{2}{*}{$\begin{array}{c}\text { Sampling } \\
\text { depth } \\
\text { (m) }\end{array}$} & \multirow[b]{2}{*}{$\begin{array}{c}\text { Chloride } \\
(\mathrm{mg} / \mathrm{L})\end{array}$} & \multirow[b]{2}{*}{$\begin{array}{c}\text { Fluoride } \\
\text { (mg/L) }\end{array}$} & \multirow[b]{2}{*}{$\begin{array}{c}\text { Silica } \\
(\mathrm{mg} / \mathrm{L})\end{array}$} & \multirow[b]{2}{*}{$\begin{array}{l}\text { Sulfate } \\
(\mathrm{mg} / \mathrm{L})\end{array}$} & \multicolumn{2}{|c|}{$\begin{array}{c}\text { Ammonia + organic } \\
\text { nitrogen }\end{array}$} & \multirow{2}{*}{$\begin{array}{c}\text { Ammonia } \\
\text { (mg/L) }\end{array}$} & \multirow[b]{2}{*}{$\begin{array}{c}\text { Nitrite + nitrate } \\
(\mathrm{mg} / \mathrm{L} \text { as N)}\end{array}$} & \multirow[b]{2}{*}{\begin{tabular}{|c|} 
Nitrite \\
(mg/L as N)
\end{tabular}} & \multirow[b]{2}{*}{$\begin{array}{c}\text { Orthophosphate } \\
\text { (mg/L as P) }\end{array}$} & \multicolumn{2}{|c|}{ Phosphorus } & \multirow{2}{*}{$\begin{array}{l}\text { Organic } \\
\text { carbon } \\
\text { (mg/L) }\end{array}$} & \multirow[b]{2}{*}{$\begin{array}{c}\text { Iron } \\
(\mu \mathrm{g} / \mathrm{L})\end{array}$} & \multirow[b]{2}{*}{$\begin{array}{c}\text { Manganese } \\
(\mu \mathrm{g} / \mathrm{L})\end{array}$} & \multirow[b]{2}{*}{\begin{tabular}{|c} 
Mercury \\
(ng/L)
\end{tabular}} \\
\hline & & & & & & & & & & & \begin{tabular}{|c|} 
Filtered \\
$(\mathrm{mg} / \mathrm{L})$
\end{tabular} & \begin{tabular}{|c|} 
Unfiltered \\
(mg/L)
\end{tabular} & & & & & $\begin{array}{c}\text { Filtered } \\
(\mathrm{mg} / \mathrm{L})\end{array}$ & \begin{tabular}{|c|} 
Unfiltered \\
(mg/L)
\end{tabular} & & & & \\
\hline 483129092462001 & Peary & 5 & 8 & 2003 & 300338 & 0.05 & & & & & & & & & & & & & & & & 0.96 \\
\hline 483109092422601 & Ryan & 5 & 8 & 2003 & 300339 & 0.05 & & & & & & & & & & & & & & & & 2.38 \\
\hline 483059092474501 & Brown & 5 & 9 & 2003 & 300340 & 0.05 & & & & & & & & & & & & & & & & 1.34 \\
\hline 483059092474501 & Brown & 5 & 19 & 2004 & 400181 & 0.05 & 0.24 & $<0.17$ & 2.41 & 1.50 & 0.35 & 0.43 & E0.02 & 0.09 & $<0.008$ & $<0.02$ & E0.004 & $<0.004$ & 7.6 & 141 & 3.4 & 1.97 \\
\hline 483129092462001 & Peary & 5 & 19 & 2004 & 400171 & 0.05 & 0.28 & $<0.17$ & 1.56 & 1.86 & 0.37 & 0.44 & $<0.04$ & $<0.06$ & $<0.008$ & $<0.02$ & 0.006 & E0.004 & 7.7 & 81 & 0.9 & 1.72 \\
\hline 483109092422601 & Ryan & 5 & 19 & 2004 & 400173 & 0.05 & 0.26 & $<0.17$ & 2.61 & 3.33 & 0.50 & 0.50 & 0.10 & $<0.06$ & $<0.008$ & $<0.02$ & 0.005 & 0.005 & 10.3 & 190 & 29.5 & 3.43 \\
\hline 483059092474501 & Brown & 8 & 5 & 2004 & 400358 & 0.05 & 0.23 & $<0.17$ & 1.06 & 1.95 & 0.38 & 0.46 & $<0.04$ & $<0.06$ & $<0.008$ & $<0.02$ & $<0.004$ & 0.005 & 8.5 & 27 & E0.4 & 1.21 \\
\hline 483129092462001 & Peary & 8 & 5 & 2004 & 400357 & 0.05 & 0.25 & $<0.17$ & 0.55 & 1.87 & 0.41 & 0.55 & $<0.04$ & $<0.06$ & $<0.008$ & $<0.02$ & E0.004 & 0.015 & 8.6 & 42 & 0.9 & 1.11 \\
\hline 483109092422601 & Ryan & 8 & 5 & 2004 & 400356 & 0.05 & 0.24 & $<0.17$ & 1.69 & 3.52 & 0.40 & 0.48 & $<0.04$ & $<0.06$ & $<0.008$ & $<0.02$ & E0.003 & 0.007 & 9.8 & 35 & E0.6 & 2.40 \\
\hline 482951092531601 & Shoepack & 8 & 25 & 2004 & 400624 & 0.05 & & & & & & & & & & & & & 15.0 & & & 2.46 \\
\hline 482951092531601 & Shoepack & 9 & 22 & 2004 & 400613 & 0.05 & 0.28 & 0.17 & 1.35 & 2.94 & 0.59 & 0.71 & $<0.04$ & $<0.06$ & $<0.008$ & $<0.02$ & 0.008 & 0.026 & 14.8 & 291 & 10.9 & 2.78 \\
\hline 483059092474501 & Brown & 9 & 23 & 2004 & 400588 & 0.05 & 0.20 & $<0.17$ & 1.06 & 1.32 & 0.36 & 0.46 & $<0.04$ & $<0.06$ & $<0.008$ & $<0.02$ & E0.002 & 0.010 & 8.3 & 47 & 1.5 & 1.59 \\
\hline 483129092462001 & Peary & 9 & 23 & 2004 & 400607 & 0.05 & 0.22 & $<0.17$ & 0.75 & 1.17 & 0.44 & 0.53 & $<0.04$ & $<0.06$ & $<0.008$ & $<0.02$ & 0.005 & 0.017 & 8.5 & 47 & 2.5 & 1.33 \\
\hline 483109092422601 & Ryan & 9 & 23 & 2004 & 400594 & 0.05 & 0.23 & $<0.17$ & 1.71 & 2.75 & 0.42 & 0.53 & $<0.04$ & $<0.06$ & $<0.008$ & $<0.02$ & E0.003 & 0.012 & 10.4 & 26 & 1.7 & 2.49 \\
\hline 482951092531601 & Shoepack & 5 & 12 & 2005 & 500325 & 0.05 & 0.34 & 0.10 & 3.31 & 3.46 & 0.52 & 0.66 & $<0.04$ & 0.08 & $<0.008$ & $<0.02$ & 0.008 & 0.023 & 15.3 & 286 & 47.2 & 3.94 \\
\hline 482951092531601 & Shoepack & 5 & 12 & 2005 & 500611 & 0.05 & & & & & & & & & & & & & 15.1 & & & 3.74 \\
\hline 483059092474501 & Brown & 5 & 13 & 2005 & 500322 & 0.05 & 0.22 & $<0.10$ & 2.98 & 1.66 & 0.38 & 0.50 & $<0.04$ & 0.07 & $<0.008$ & $<0.02$ & 0.006 & 0.011 & 9.6 & 173 & 13.8 & 2.56 \\
\hline 483129092462001 & Peary & 5 & 13 & 2005 & 500323 & 0.05 & 0.23 & $<0.10$ & 2.17 & 1.58 & 0.37 & 0.49 & $<0.04$ & $<0.06$ & $<0.008$ & $<0.02$ & 0.006 & 0.024 & 9.3 & 145 & 0.7 & 2.01 \\
\hline 483109092422601 & Ryan & 5 & 13 & 2005 & 500324 & 0.05 & 0.24 & $<0.10$ & 3.58 & 3.28 & 0.48 & 0.57 & 0.08 & E0.05 & $<0.008$ & $<0.02$ & 0.006 & 0.011 & 11.2 & 178 & 29.1 & 3.07 \\
\hline 483059092474501 & Brown & 7 & 13 & 2005 & 500388 & 0.05 & & & & & & & & & & & & & 10.1 & & & 2.24 \\
\hline 483129092462001 & Peary & 7 & 13 & 2005 & 500389 & 0.05 & & & & & & & & & & & & & 9.8 & & & 1.77 \\
\hline 482951092531601 & Shoepack & 7 & 14 & 2005 & 500390 & 0.05 & & & & & & & & & & & & & 15.3 & & & 3.69 \\
\hline 483109092422601 & Ryan & 7 & 15 & 2005 & 500612 & 0.05 & & & & & & & & & & & & & 12.0 & & & 2.35 \\
\hline 483059092474501 & Brown & 9 & 14 & 2005 & 500528 & 0.05 & & & & & & & & & & & & & & & & 2.04 \\
\hline 483129092462001 & Peary & 9 & 14 & 2005 & 500529 & 0.05 & & & & & & & & & & & & & & & & 1.09 \\
\hline 483109092422601 & Ryan & 9 & 14 & 2005 & 500527 & 0.05 & & & & & & & & & & & & & & & & 2.55 \\
\hline 483059092474501 & Brown & 5 & 16 & 2006 & 600190 & 0.05 & & & & & & & & & & & & & 8.6 & & & 1.96 \\
\hline 483129092462001 & Peary & 5 & 16 & 2006 & 600189 & 0.05 & & & & & & & & & & & & & & & & 1.53 \\
\hline 483109092422601 & Ryan & 5 & 16 & 2006 & |600192 & 0.05 & & & & & & & & & & & & & 6.5 & & & 3.02 \\
\hline 482951092531601 & Shoepack & 5 & 17 & 2006 & 600191 & 0.05 & & & & & & & & & & & & & 14.7 & & & 3.58 \\
\hline 482951092531601 & Shoepack & 7 & $27 \mid$ & 2006 & 600710 & 0.05 & & & & & & & & & & & & & & & & 2.07 \\
\hline 483059092474501 & Brown & 7 & 28 & 2006 & 600712 & 0.05 & & & & & & & & & & & & & & & & 1.58 \\
\hline 483129092462001 & Peary & 7 & 28 & 2006 & 600711 & 0.05 & & & & & & & & & & & & & & & & 0.86 \\
\hline 483109092422601 & Ryan & 7 & 28 & 2006 & 600708 & 0.05 & & & & & & & & & & & & & & & & 2.85 \\
\hline 482805092484101 & Agnes & 8 & 2 & 2006 & 600709 & 0.05 & & & & & & & & & & & & & & & & 3.05 \\
\hline 482951092531601 & Shoepack & 9 & 15 & 2006 & 500526 & 0.05 & & & & & & & & & & & & & & & & 2.24 \\
\hline
\end{tabular}




\begin{tabular}{|c|c|c|c|c|c|c|c|}
\hline \multirow[b]{2}{*}{ Station no. } & \multirow[b]{2}{*}{ Lake } & \multicolumn{3}{|c|}{ Collection Date } & \multirow{2}{*}{$\begin{array}{c}\text { Sample } \\
\text { no. }\end{array}$} & \multirow{2}{*}{$\begin{array}{c}\text { Sampling } \\
\text { depth } \\
\text { (m) }\end{array}$} & \multirow{2}{*}{$\begin{array}{l}\text { Methylmercury } \\
\text { (ng/L) }\end{array}$} \\
\hline & & Month & Day & Year & & & \\
\hline 483129092462001 & Peary & 5 & 8 & 2003 & 300338 & 0.05 & 0.07 \\
\hline 483109092422601 & Ryan & 5 & 8 & 2003 & 300339 & 0.05 & 0.12 \\
\hline 483059092474501 & Brown & 5 & 9 & 2003 & 300340 & 0.05 & 0.06 \\
\hline 483059092474501 & Brown & 5 & 19 & 2004 & 400181 & 0.05 & $<0.04$ \\
\hline 483129092462001 & Peary & 5 & 19 & 2004 & 400171 & 0.05 & $<0.04$ \\
\hline 483109092422601 & Ryan & 5 & 19 & 2004 & 400173 & 0.05 & 0.16 \\
\hline 483059092474501 & Brown & 8 & 5 & 2004 & 400358 & 0.05 & $<0.04$ \\
\hline 483129092462001 & Peary & 8 & 5 & 2004 & 400357 & 0.05 & 0.07 \\
\hline 483109092422601 & Ryan & 8 & 5 & 2004 & 400356 & 0.05 & 0.11 \\
\hline 482951092531601 & Shoepack & 8 & 25 & 2004 & 400624 & 0.05 & 0.20 \\
\hline 482951092531601 & Shoepack & 9 & 22 & 2004 & 400613 & 0.05 & 0.11 \\
\hline 483059092474501 & Brown & 9 & 23 & 2004 & 400588 & 0.05 & 0.06 \\
\hline 483129092462001 & Peary & 9 & 23 & 2004 & 400607 & 0.05 & $<0.04$ \\
\hline 483109092422601 & Ryan & 9 & 23 & 2004 & 400594 & 0.05 & 0.08 \\
\hline 482951092531601 & Shoepack & 5 & 12 & 2005 & 500325 & 0.05 & 0.24 \\
\hline 482951092531601 & Shoepack & 5 & 12 & 2005 & 500611 & 0.05 & 0.26 \\
\hline 483059092474501 & Brown & 5 & 13 & 2005 & 500322 & 0.05 & 0.16 \\
\hline 483129092462001 & Peary & 5 & 13 & 2005 & 500323 & 0.05 & 0.10 \\
\hline 483109092422601 & Ryan & 5 & 13 & 2005 & 500324 & 0.05 & 0.19 \\
\hline 483059092474501 & Brown & 7 & 13 & 2005 & 500388 & 0.05 & 0.17 \\
\hline 483129092462001 & Peary & 7 & 13 & 2005 & 500389 & 0.05 & 0.07 \\
\hline 482951092531601 & Shoepack & 7 & 14 & 2005 & 500390 & 0.05 & 0.20 \\
\hline 483109092422601 & Ryan & 7 & 15 & 2005 & 500612 & 0.05 & 0.10 \\
\hline 483059092474501 & Brown & 9 & 14 & 2005 & 500528 & 0.05 & 0.10 \\
\hline 483129092462001 & Peary & 9 & 14 & 2005 & 500529 & 0.05 & $<0.04$ \\
\hline 483109092422601 & Ryan & 9 & 14 & 2005 & 500527 & 0.05 & 0.15 \\
\hline 483059092474501 & Brown & 5 & 16 & 2006 & 600190 & 0.05 & 0.11 \\
\hline 483129092462001 & Peary & 5 & 16 & 2006 & 600189 & 0.05 & 0.06 \\
\hline 483109092422601 & Ryan & 5 & 16 & 2006 & 600192 & 0.05 & 0.11 \\
\hline 482951092531601 & Shoepack & 5 & 17 & 2006 & 600191 & 0.05 & 0.18 \\
\hline 482951092531601 & Shoepack & 7 & 27 & 2006 & 600710 & 0.05 & 0.26 \\
\hline 483059092474501 & Brown & 7 & 28 & 2006 & 600712 & 0.05 & 0.11 \\
\hline 483129092462001 & Peary & 7 & 28 & 2006 & 600711 & 0.05 & 0.06 \\
\hline 483109092422601 & Ryan & 7 & 28 & 2006 & 600708 & 0.05 & 0.10 \\
\hline 482805092484101 & Agnes & 8 & 2 & 2006 & 600709 & 0.05 & 0.26 \\
\hline 482951092531601 & Shoepack & 9 & 15 & 2006 & 500526 & 0.05 & 0.16 \\
\hline
\end{tabular}


Click back to Contents

Appendix 9-Mercury in Age-1 Yellow Perch in Selected Lakes at Voyageurs National Park, MN

\begin{tabular}{|c|c|c|c|c|c|c|c|c|c|c|}
\hline \multirow[b]{2}{*}{ Sample ID } & \multirow[b]{2}{*}{ Lake } & \multicolumn{3}{|c|}{ Collection date } & \multirow{2}{*}{$\begin{array}{l}\text { Total length } \\
(\mathrm{mm})\end{array}$} & \multirow{2}{*}{$\begin{array}{c}\text { Fish } \\
\text { wet weight } \\
\text { (g) }\end{array}$} & $\begin{array}{c}\text { Total mercury } \\
\text { wet weight }\end{array}$ & \begin{tabular}{c|} 
Fish \\
dry weight
\end{tabular} & $\begin{array}{l}\text { Total mercury } \\
\text { dry weight }\end{array}$ & Age \\
\hline & & Month & Day & Year & & & & & & (years) \\
\hline br03yp019 & Brown & 5 & 12 & 2003 & 54 & 1.42 & 71 & 0.299 & 338 & 1 \\
\hline br03yp026 & Brown & 5 & 12 & 2003 & 58 & 1.56 & 77 & 0.331 & 361 & 1 \\
\hline br03yp011 & Brown & 5 & 12 & 2003 & 56 & 1.58 & 76 & 0.331 & 364 & 1 \\
\hline br03yp041 & Brown & 5 & 12 & 2003 & 50 & 1.13 & 72 & 0.221 & 367 & 1 \\
\hline br03yp027 & Brown & 5 & 12 & 2003 & 54 & 1.31 & 74 & 0.262 & 368 & 1 \\
\hline br03yp034 & Brown & 5 & 12 & 2003 & 54 & 1.36 & 80 & 0.293 & 369 & 1 \\
\hline br03yp021 & Brown & 5 & 12 & 2003 & 53 & 1.50 & 78 & 0.312 & 373 & 1 \\
\hline br03yp018 & Brown & 5 & 12 & 2003 & 55 & 1.46 & 76 & 0.296 & 375 & 1 \\
\hline br03yp013 & Brown & 5 & 12 & 2003 & 63 & 2.12 & 81 & 0.456 & 377 & 1 \\
\hline br03yp017 & Brown & 5 & 12 & 2003 & 57 & 1.58 & 78 & 0.320 & 385 & 1 \\
\hline br03yp015 & Brown & 5 & 12 & 2003 & 55 & 1.42 & 82 & 0.294 & 396 & 1 \\
\hline br03yp043 & Brown & 5 & 12 & 2003 & 49 & 1.15 & 81 & 0.236 & 397 & 1 \\
\hline br03yp012 & Brown & 5 & 12 & 2003 & 56 & 1.46 & 80 & 0.292 & 399 & 1 \\
\hline br03yp006 & Brown & 5 & 12 & 2003 & 59 & 1.95 & 88 & 0.430 & 401 & 1 \\
\hline br03yp044 & Brown & 5 & 12 & 2003 & 58 & 1.57 & 84 & 0.325 & 404 & 1 \\
\hline br03yp030 & Brown & 5 & 12 & 2003 & 57 & 1.69 & 89 & 0.371 & 406 & 1 \\
\hline br03yp025 & Brown & 5 & 12 & 2003 & 54 & 1.21 & 83 & 0.247 & 407 & 1 \\
\hline br03yp005 & Brown & 5 & 12 & 2003 & 53 & 1.27 & 81 & 0.249 & 411 & 1 \\
\hline br03yp004 & Brown & 5 & 12 & 2003 & 59 & 1.84 & 89 & 0.395 & 415 & 1 \\
\hline br03yp020 & Brown & 5 & 12 & 2003 & 58 & 1.85 & 77 & 0.342 & 417 & 1 \\
\hline br03yp022 & Brown & 5 & 12 & 2003 & 59 & 1.74 & 86 & 0.355 & 419 & 1 \\
\hline br03yp023 & Brown & 5 & 12 & 2003 & 53 & 1.32 & 83 & 0.257 & 425 & 1 \\
\hline br03yp039 & Brown & 5 & 12 & 2003 & 53 & 1.40 & 86 & 0.280 & 430 & 1 \\
\hline br03yp046 & Brown & 5 & 12 & 2003 & 58 & 1.85 & 90 & 0.381 & 436 & 1 \\
\hline br03yp042 & Brown & 5 & 12 & 2003 & 53 & 1.31 & 87 & 0.261 & 437 & 1 \\
\hline br03yp024 & Brown & 5 & 12 & 2003 & 52 & 1.24 & 82 & 0.231 & 438 & 1 \\
\hline br03yp014 & Brown & 5 & 12 & 2003 & 55 & 1.49 & 89 & 0.304 & 438 & 1 \\
\hline br03yp008 & Brown & 5 & 12 & 2003 & 54 & 1.33 & 90 & 0.270 & 444 & 1 \\
\hline br03yp016 & Brown & 5 & 12 & 2003 & 53 & 1.40 & 86 & 0.270 & 445 & 1 \\
\hline br03yp029 & Brown & 5 & 12 & 2003 & 57 & 1.79 & 96 & 0.385 & 445 & 1 \\
\hline br03yp007 & Brown & 5 & 12 & 2003 & 52 & 1.32 & 91 & 0.268 & 448 & 1 \\
\hline br03yp050 & Brown & 5 & 12 & 2003 & 49 & 1.16 & 94 & 0.242 & 452 & 1 \\
\hline br03yp035 & Brown & 5 & 12 & 2003 & 54 & 1.27 & 90 & 0.252 & 453 & 1 \\
\hline br03yp037 & Brown & 5 & 12 & 2003 & 52 & 1.14 & 83 & 0.207 & 456 & 1 \\
\hline br03yp009 & Brown & 5 & 12 & 2003 & 59 & 1.68 & 91 & 0.332 & 463 & 1 \\
\hline br03yp010 & Brown & 5 & 12 & 2003 & 50 & 1.09 & 89 & 0.205 & 473 & 1 \\
\hline br03yp051 & Brown & 5 & 12 & 2003 & 55 & 1.32 & 96 & 0.267 & 473 & 1 \\
\hline br03yp028 & Brown & 5 & 12 & 2003 & 54 & 1.37 & 104 & 0.289 & 493 & 1 \\
\hline br03yp045 & Brown & 5 & 12 & 2003 & 57 & 1.51 & 103 & 0.309 & 505 & 1 \\
\hline br03yp038 & Brown & 5 & 12 & 2003 & 54 & 1.48 & 104 & 0.298 & 515 & 1 \\
\hline br03yp036 & Brown & 5 & 12 & 2003 & 55 & 1.47 & 109 & 0.310 & 516 & 1 \\
\hline br03yp040 & Brown & 5 & 12 & 2003 & 54 & 1.17 & 101 & 0.226 & 525 & 1 \\
\hline br03yp031 & Brown & 5 & 12 & 2003 & 57 & 1.58 & 111 & 0.334 & 526 & 1 \\
\hline br03yp032 & Brown & 5 & 12 & 2003 & 49 & 1.17 & 111 & 0.244 & 530 & 1 \\
\hline br03yp047 & Brown & 5 & 12 & 2003 & 58 & 1.62 & 106 & 0.319 & 539 & 1 \\
\hline br03yp033 & Brown & 5 & 12 & 2003 & 62 & 2.02 & 115 & 0.427 & 543 & 1 \\
\hline br03yp049 & Brown & 5 & 12 & 2003 & 53 & 1.36 & 107 & 0.262 & 553 & 1 \\
\hline ре03ур029 & Peary & 5 & 8 & 2003 & 61 & 2.11 & 47 & 0.456 & 216 & 1 \\
\hline ре03ур023 & Peary & 5 & 8 & 2003 & 58 & 1.71 & 50 & 0.349 & 247 & 1 \\
\hline ре03yp016 & Peary & 5 & 8 & 2003 & 52 & 1.28 & 50 & 0.248 & 257 & 1 \\
\hline pe03yp026 & Peary & 5 & 8 & 2003 & 56 & 1.55 & 53 & 0.312 & 261 & 1 \\
\hline ре0Зур019 & Peary & 5 & 8 & 2003 & 52 & 1.31 & 52 & 0.256 & 266 & 1 \\
\hline pe03yp004 & Peary & 5 & 8 & 2003 & 55 & 1.65 & 55 & 0.341 & 268 & 1 \\
\hline ре03ур021 & Peary & 5 & 8 & 2003 & 64 & 2.38 & 58 & 0.515 & 270 & 1 \\
\hline ре03ур006 & Peary & 5 & 8 & 2003 & 47 & 0.95 & 53 & 0.181 & 279 & 1 \\
\hline ре0Зур001 & Peary & 5 & 8 & 2003 & 53 & 1.52 & 60 & 0.312 & 294 & 1 \\
\hline ре03ур002 & Peary & 5 & 8 & 2003 & 57 & 1.67 & 58 & 0.326 & 295 & 1 \\
\hline pe03yp020 & Peary & 5 & 8 & 2003 & 47 & 1.03 & 57 & 0.197 & 300 & 1 \\
\hline ре0Зур025 & Peary & 5 & 8 & 2003 & 47 & 1.06 & 60 & 0.206 & 306 & 1 \\
\hline pe03yp007 & Peary & 5 & 8 & 2003 & 54 & 1.35 & 62 & 0.265 & 318 & 1 \\
\hline ре0Зур010 & Peary & 5 & 8 & 2003 & 46 & 0.91 & 66 & 0.179 & 335 & 1 \\
\hline ре03ур011 & Peary & 5 & 8 & 2003 & 51 & 1.24 & 67 & 0.247 & 336 & 1 \\
\hline ре03ур030 & Peary & 5 & 8 & 2003 & 50 & 1.13 & 70 & 0.228 & 345 & 1 \\
\hline ре03yp022 & Peary & 5 & 8 & 2003 & 53 & 1.34 & 72 & 0.275 & 349 & 1 \\
\hline pe03yp009 & Peary & 5 & 8 & 2003 & 54 & 1.18 & 70 & 0.235 & 352 & 1 \\
\hline ре0Зур013 & Peary & 5 & 8 & 2003 & 52 & 1.39 & 70 & 0.273 & 354 & 1 \\
\hline ре03ур003 & Peary & 5 & 8 & 2003 & 52 & 1.25 & 70 & 0.246 & 357 & 1 \\
\hline ре03ур024 & Peary & 5 & 8 & 2003 & 48 & 0.98 & 69 & 0.187 & 361 & 1 \\
\hline ре03ур014 & Peary & 5 & 8 & 2003 & 50 & 1.14 & 72 & 0.224 & 364 & 1 \\
\hline ре0Зур005 & Peary & 5 & 8 & 2003 & 49 & 1.12 & 72 & 0.215 & 377 & 1 \\
\hline ре03ур008 & Peary & 5 & 8 & 2003 & 47 & 0.96 & 74 & 0.188 & 377 & 1 \\
\hline ре03ур015 & Peary & 5 & 8 & 2003 & 48 & 0.95 & 68 & 0.168 & 384 & 1 \\
\hline ре03ур018 & Peary & 5 & 8 & 2003 & 48 & 1.02 & 72 & 0.189 & 389 & 1 \\
\hline ре03yp012 & Peary & 5 & 8 & 2003 & 45 & 0.77 & 73 & 0.144 & 391 & 1 \\
\hline ре0Зур027 & Peary & 5 & 8 & 2003 & 46 & 0.81 & 79 & 0.160 & 398 & 1 \\
\hline pe03yp017 & Peary & 5 & 8 & 2003 & 49 & 0.90 & 74 & 0.161 & 415 & \\
\hline
\end{tabular}




\begin{tabular}{|c|c|c|c|c|c|c|c|c|c|c|}
\hline \multirow[b]{2}{*}{ Sample ID } & \multirow[b]{2}{*}{ Lake } & \multicolumn{3}{|c|}{ Collection date } & \multirow{2}{*}{$\begin{array}{c}\text { Total length } \\
(\mathrm{mm})\end{array}$} & \multirow{2}{*}{$\begin{array}{c}\text { Fish } \\
\text { wet weight } \\
\text { (g) }\end{array}$} & $\begin{array}{c}\text { Total mercury } \\
\text { wet weight }\end{array}$ & $\begin{array}{c}\text { Fish } \\
\text { dry weight }\end{array}$ & $\begin{array}{l}\text { Total mercury } \\
\text { dry weight }\end{array}$ & Age \\
\hline & & Month & Day & Year & & & & & & (years) \\
\hline ре03ур028 & Peary & 5 & 8 & 2003 & 49 & 1.07 & 79 & 0.203 & 417 & 1 \\
\hline ry03yp023 & Ryan & 5 & 12 & 2003 & 57 & 1.36 & 59 & 0.266 & 301 & 1 \\
\hline ry03yp020 & Ryan & 5 & 12 & 2003 & 58 & 1.61 & 87 & 0.319 & 440 & 1 \\
\hline ry03yp005 & Ryan & 5 & 12 & 2003 & 54 & 1.37 & 100 & 0.247 & 555 & 1 \\
\hline ry03yp025 & Ryan & 5 & 12 & 2003 & 58 & 1.68 & 109 & 0.320 & 570 & 1 \\
\hline ry03yp017 & Ryan & 5 & 12 & 2003 & 53 & 1.20 & 105 & 0.220 & 572 & 1 \\
\hline ry03yp030 & Ryan & 5 & 12 & 2003 & 67 & 2.30 & 111 & 0.415 & 614 & 1 \\
\hline ry03yp010 & Ryan & 5 & 12 & 2003 & 63 & 2.04 & 126 & 0.409 & 627 & 1 \\
\hline ry03yp027 & Ryan & 5 & 12 & 2003 & 56 & 1.09 & 110 & 0.188 & 639 & 1 \\
\hline ry03yp029 & Ryan & 5 & 12 & 2003 & 60 & 1.87 & 126 & 0.351 & 669 & 1 \\
\hline ry03yp012 & Ryan & 5 & 12 & 2003 & 53 & 1.23 & 125 & 0.226 & 680 & 1 \\
\hline ry03yp001 & Ryan & 5 & 12 & 2003 & 74 & 3.81 & 144 & 0.773 & 709 & 1 \\
\hline ry03yp008 & Ryan & 5 & 12 & 2003 & 54 & 1.32 & 145 & 0.256 & 746 & 1 \\
\hline ry03yp003 & Ryan & 5 & 12 & 2003 & 53 & 1.09 & 143 & 0.208 & 748 & 1 \\
\hline ry03yp019 & Ryan & 5 & 12 & 2003 & 50 & 1.02 & 146 & 0.199 & 749 & 1 \\
\hline rу03ур016 & Ryan & 5 & 12 & 2003 & 50 & 1.04 & 189 & 0.191 & 1,028 & 1 \\
\hline ry03yp015 & Ryan & 5 & 12 & 2003 & 50 & 1.04 & 189 & 0.190 & 1,034 & 1 \\
\hline ry03yp021 & Ryan & 5 & 12 & 2003 & 52 & 1.27 & 227 & 0.253 & 1,138 & 1 \\
\hline ry03yp007 & Ryan & 5 & 12 & 2003 & 49 & 0.95 & 218 & 0.180 & 1,149 & 1 \\
\hline ry03yp018 & Ryan & 5 & 12 & 2003 & 50 & 1.03 & 217 & 0.194 & 1,151 & 1 \\
\hline ry03yp011 & Ryan & 5 & 12 & 2003 & 49 & 1.03 & 222 & 0.186 & 1,230 & 1 \\
\hline ry03yp013 & Ryan & 5 & 12 & 2003 & 50 & 0.96 & 165 & 0.127 & 1,245 & 1 \\
\hline ry03yp006 & Ryan & 5 & 12 & 2003 & 47 & 0.78 & 232 & 0.142 & 1,277 & 1 \\
\hline ry03yp002 & Ryan & 5 & 12 & 2003 & 63 & 2.08 & 266 & 0.411 & 1,347 & 1 \\
\hline ry03yp004 & Ryan & 5 & 12 & 2003 & 47 & 0.81 & 262 & 0.157 & 1,354 & 1 \\
\hline ry03yp022 & Ryan & 5 & 12 & 2003 & 48 & 0.87 & 253 & 0.153 & 1,440 & 1 \\
\hline ry03yp009 & Ryan & 5 & 12 & 2003 & 51 & 1.01 & 276 & 0.191 & 1,460 & 1 \\
\hline ry03yp026 & Ryan & 5 & 12 & 2003 & 47 & 0.73 & 285 & 0.142 & 1,468 & 1 \\
\hline ry03yp024 & Ryan & 5 & 12 & 2003 & 57 & 1.52 & 274 & 0.278 & 1,498 & 1 \\
\hline ry03yp014 & Ryan & 5 & 12 & 2003 & 57 & 1.50 & 297 & 0.282 & 1,579 & 1 \\
\hline br04yp021 & Brown & 5 & 18 & 2004 & 54 & 1.19 & 42 & 0.234 & 211 & 1 \\
\hline br04yp018 & Brown & 5 & 18 & 2004 & 48 & 1.00 & 45 & 0.205 & 221 & 1 \\
\hline br04yp020 & Brown & 5 & 18 & 2004 & 49 & 1.08 & 46 & 0.216 & 231 & 1 \\
\hline br04yp026 & Brown & 5 & 18 & 2004 & 51 & 1.16 & 48 & 0.238 & 233 & 1 \\
\hline br04yp004 & Brown & 5 & 18 & 2004 & 49 & 1.07 & 47 & 0.215 & 235 & 1 \\
\hline br04yp012 & Brown & 5 & 18 & 2004 & 50 & 1.26 & 53 & 0.275 & 245 & 1 \\
\hline br04yp008 & Brown & 5 & 18 & 2004 & 49 & 1.10 & 52 & 0.232 & 248 & 1 \\
\hline br04yp028 & Brown & 5 & 18 & 2004 & 53 & 1.19 & 49 & 0.233 & 253 & 1 \\
\hline br04yp017 & Brown & 5 & 18 & 2004 & 53 & 1.12 & 52 & 0.231 & 253 & 1 \\
\hline br04yp003 & Brown & 5 & 18 & 2004 & 48 & 1.00 & 52 & 0.204 & 256 & 1 \\
\hline br04yp027 & Brown & 5 & 18 & 2004 & 51 & 1.06 & 55 & 0.225 & 261 & 1 \\
\hline br04yp010 & Brown & 5 & 18 & 2004 & 50 & 1.13 & 54 & 0.227 & 267 & 1 \\
\hline br04yp016 & Brown & 5 & 18 & 2004 & 47 & 0.94 & 55 & 0.192 & 271 & 1 \\
\hline br04yp011 & Brown & 5 & 18 & 2004 & 47 & 0.93 & 54 & 0.183 & 274 & 1 \\
\hline br04yp005 & Brown & 5 & 18 & 2004 & 49 & 1.14 & 57 & 0.233 & 277 & 1 \\
\hline br04yp019 & Brown & 5 & 18 & 2004 & 47 & 0.91 & 56 & 0.180 & 281 & 1 \\
\hline br04yp002 & Brown & 5 & 18 & 2004 & 47 & 0.98 & 55 & 0.190 & 286 & 1 \\
\hline br04yp030 & Brown & 5 & 18 & 2004 & 50 & 1.27 & 58 & 0.254 & 288 & 1 \\
\hline br04yp006 & Brown & 5 & 18 & 2004 & 47 & 0.93 & 57 & 0.185 & 289 & 1 \\
\hline br04yp014 & Brown & 5 & 18 & 2004 & 47 & 0.94 & 61 & 0.193 & 296 & 1 \\
\hline br04yp013 & Brown & 5 & 18 & 2004 & 49 & 1.07 & 60 & 0.216 & 296 & 1 \\
\hline br04yp015 & Brown & 5 & 18 & 2004 & 49 & 1.03 & 60 & 0.207 & 297 & 1 \\
\hline br04yp024 & Brown & 5 & 18 & 2004 & 54 & 1.29 & 61 & 0.261 & 300 & 1 \\
\hline br04yp029 & Brown & 5 & 18 & 2004 & 52 & 1.23 & 60 & 0.244 & 301 & 1 \\
\hline br04yp023 & Brown & 5 & 18 & 2004 & 52 & 1.24 & 60 & 0.243 & 307 & 1 \\
\hline br04yp007 & Brown & 5 & 18 & 2004 & 43 & 0.75 & 65 & 0.155 & 315 & 1 \\
\hline br04yp009 & Brown & 5 & 18 & 2004 & 51 & 0.96 & 70 & 0.181 & 370 & 1 \\
\hline br04yp022 & Brown & 5 & 18 & 2004 & 50 & 1.07 & 81 & 0.225 & 385 & 1 \\
\hline br04yp025 & Brown & 5 & 18 & 2004 & 55 & 1.41 & 79 & 0.281 & 394 & 1 \\
\hline br04yp001 & Brown & 5 & 18 & 2004 & 49 & 0.82 & 69 & 0.141 & 401 & 1 \\
\hline ре04ур011 & Peary & 5 & 19 & 2004 & 59 & 1.93 & 26 & 0.419 & 121 & 1 \\
\hline pe04yp002 & Peary & 5 & 19 & 2004 & 60 & 2.06 & 25 & 0.428 & 122 & 1 \\
\hline ре04ур022 & Peary & 5 & 19 & 2004 & 59 & 1.96 & 25 & 0.397 & 123 & 1 \\
\hline ре04yp010 & Peary & 5 & 19 & 2004 & 58 & 1.70 & 29 & 0.366 & 137 & 1 \\
\hline ре04ур017 & Peary & 5 & 19 & 2004 & 59 & 1.83 & 28 & 0.369 & 141 & 1 \\
\hline ре04yp019 & Peary & 5 & 19 & 2004 & 60 & 2.09 & 31 & 0.454 & 144 & 1 \\
\hline ре04yp023 & Peary & 5 & 19 & 2004 & 60 & 2.02 & 31 & 0.432 & 145 & 1 \\
\hline ре04yp008 & Peary & 5 & 19 & 2004 & 60 & 2.00 & 31 & 0.422 & 146 & 1 \\
\hline ре04yp001 & Peary & 5 & 19 & 2004 & 56 & 1.49 & 28 & 0.283 & 146 & 1 \\
\hline ре04ур013 & Peary & 5 & 19 & 2004 & 57 & 1.87 & 32 & 0.404 & 149 & 1 \\
\hline ре04yp016 & Peary & 5 & 19 & 2004 & 57 & 1.67 & 30 & 0.339 & 149 & 1 \\
\hline рe04yp021 & Peary & 5 & 19 & 2004 & 58 & 1.89 & 33 & 0.408 & 154 & 1 \\
\hline ре04yp009 & Peary & 5 & 19 & 2004 & 60 & 2.03 & 34 & 0.440 & 156 & 1 \\
\hline ре04ур015 & Peary & 5 & 19 & 2004 & 60 & 2.22 & 34 & 0.473 & 158 & 1 \\
\hline ре04ур014 & Peary & 5 & 19 & 2004 & 57 & 1.73 & 35 & 0.379 & 159 & 1 \\
\hline pe04yp003 & Peary & 5 & 19 & 2004 & 57 & 1.56 & 32 & 0.306 & 161 & \\
\hline
\end{tabular}




\begin{tabular}{|c|c|c|c|c|c|c|c|c|c|c|}
\hline \multirow[b]{2}{*}{ Sample ID } & \multirow[b]{2}{*}{ Lake } & \multicolumn{3}{|c|}{ Collection date } & \multirow{2}{*}{$\begin{array}{l}\text { Total length } \\
\text { (mm) }\end{array}$} & \multirow{2}{*}{\begin{tabular}{|c|} 
Fish \\
wet weight \\
(g)
\end{tabular}} & $\begin{array}{c}\text { Total mercury } \\
\text { wet weight }\end{array}$ & $\begin{array}{c}\text { Fish } \\
\text { dry weight }\end{array}$ & $\begin{array}{l}\text { Total mercury } \\
\text { dry weight }\end{array}$ & Age \\
\hline & & Month & Day & \begin{tabular}{|l|} 
Year \\
\end{tabular} & & & & & & (years) \\
\hline pe04yp020 & Peary & 5 & 19 & 2004 & 58 & 1.68 & 33 & 0.343 & 162 & 1 \\
\hline ре04yp004 & Peary & 5 & 19 & 2004 & 56 & 1.72 & 33 & 0.345 & 165 & 1 \\
\hline ре04ур006 & Peary & 5 & 19 & 2004 & 58 & 1.89 & 34 & 0.389 & 165 & 1 \\
\hline pe04yp005 & Peary & 5 & 19 & 2004 & 57 & 1.64 & 34 & 0.333 & 165 & 1 \\
\hline pe04yp007 & Peary & 5 & 19 & 2004 & 59 & 1.84 & 35 & 0.387 & 168 & 1 \\
\hline ре04yp018 & Peary & 5 & 19 & 2004 & 57 & 1.54 & 34 & 0.304 & 172 & 1 \\
\hline ре04yp012 & Peary & 5 & 19 & 2004 & 58 & 1.71 & 35 & 0.349 & 173 & 1 \\
\hline ry04yp021 & Ryan & 5 & 18 & 2004 & 65 & 2.41 & 141 & 0.503 & 675 & 1 \\
\hline ry04yp026 & Ryan & 5 & 18 & 2004 & 68 & 2.49 & 140 & 0.509 & 683 & 1 \\
\hline ry04yp019 & Ryan & 5 & 18 & 2004 & 65 & 2.37 & 142 & 0.486 & 694 & 1 \\
\hline ry04yp028 & Ryan & 5 & 18 & 2004 & 65 & 2.40 & 155 & 0.519 & 716 & 1 \\
\hline ry04yp012 & Ryan & 5 & 18 & 2004 & 64 & 2.45 & 154 & 0.528 & 716 & 1 \\
\hline ry04yp006 & Ryan & 5 & 18 & 2004 & 64 & 2.14 & 159 & 0.456 & 745 & 1 \\
\hline ry04yp011 & Ryan & 5 & 18 & 2004 & 65 & 2.34 & 167 & 0.511 & 766 & 1 \\
\hline ry04yp018 & Ryan & 5 & 18 & 2004 & 65 & 2.34 & 175 & 0.521 & 784 & 1 \\
\hline ry04yp017 & Ryan & 5 & 18 & 2004 & 67 & 2.52 & 167 & 0.537 & 786 & 1 \\
\hline ry04yp027 & Ryan & 5 & 18 & 2004 & 68 & 2.55 & 166 & 0.537 & 788 & 1 \\
\hline ry04yp022 & Ryan & 5 & 18 & 2004 & 66 & 2.41 & 159 & 0.487 & 788 & 1 \\
\hline ry04yp016 & Ryan & 5 & 18 & 2004 & 63 & 2.21 & 169 & 0.474 & 789 & 1 \\
\hline ry04yp009 & Ryan & 5 & 18 & 2004 & 65 & 2.57 & 169 & 0.547 & 795 & 1 \\
\hline ry04yp007 & Ryan & 5 & 18 & 2004 & 67 & 2.49 & 167 & 0.518 & 803 & 1 \\
\hline ry04yp015 & Ryan & 5 & 18 & 2004 & 63 & 2.05 & 180 & 0.445 & 828 & 1 \\
\hline ry04yp023 & Ryan & 5 & 18 & 2004 & 65 & 2.21 & 189 & 0.467 & 893 & 1 \\
\hline ry04yp029 & Ryan & 5 & 18 & 2004 & 66 & 2.37 & 188 & 0.498 & 895 & 1 \\
\hline ry04yp010 & Ryan & 5 & 18 & 2004 & 63 & 2.09 & 184 & 0.429 & 899 & 1 \\
\hline ry04yp008 & Ryan & 5 & 18 & 2004 & 67 & 2.02 & 180 & 0.401 & 906 & 1 \\
\hline ry04yp003 & Ryan & 5 & 18 & 2004 & 63 & 2.09 & 191 & 0.441 & 906 & 1 \\
\hline ry04yp024 & Ryan & 5 & 18 & 2004 & 68 & 2.83 & 199 & 0.614 & 917 & 1 \\
\hline ry04yp025 & Ryan & 5 & 18 & 2004 & 65 & 2.36 & 202 & 0.516 & 926 & 1 \\
\hline ry04yp004 & Ryan & 5 & 18 & 2004 & 64 & 2.15 & 194 & 0.448 & 929 & 1 \\
\hline ry04yp020 & Ryan & 5 & 18 & 2004 & 67 & 2.62 & 198 & 0.549 & 944 & 1 \\
\hline ry04yp002 & Ryan & 5 & 18 & 2004 & 77 & 3.48 & 195 & 0.719 & 946 & 1 \\
\hline ry04yp005 & Ryan & 5 & 18 & 2004 & 65 & 2.45 & 206 & 0.507 & 993 & 1 \\
\hline ry04yp014 & Ryan & 5 & 18 & 2004 & 60 & 1.71 & 208 & 0.349 & 1,018 & 1 \\
\hline ry04yp013 & Ryan & 5 & 18 & 2004 & 63 & 2.06 & 211 & 0.416 & 1,045 & 1 \\
\hline ry04yp030 & Ryan & 5 & 18 & 2004 & 79 & 2.60 & 258 & 0.590 & 1,137 & 1 \\
\hline br05yp016 & Brown & 5 & 16 & 2005 & 60 & 1.81 & 57 & 0.388 & 264 & 1 \\
\hline br05yp010 & Brown & 5 & 16 & 2005 & 60 & 1.99 & 57 & 0.411 & 274 & 1 \\
\hline br05yp008 & Brown & 5 & 16 & 2005 & 50 & 1.13 & 59 & 0.244 & 275 & 1 \\
\hline br05yp035 & Brown & 5 & 16 & 2005 & 55 & 1.55 & 58 & 0.320 & 282 & 1 \\
\hline br05yp031 & Brown & 5 & 16 & 2005 & 46 & 0.91 & 57 & 0.169 & 305 & 1 \\
\hline br05yp009 & Brown & 5 & 16 & 2005 & 53 & 1.33 & 64 & 0.274 & 313 & 1 \\
\hline br05yp003 & Brown & 5 & 16 & 2005 & 49 & 1.01 & 65 & 0.209 & 313 & 1 \\
\hline br05yp018 & Brown & 5 & 16 & 2005 & 53 & 1.30 & 63 & 0.261 & 313 & 1 \\
\hline br05yp007 & Brown & 5 & 16 & 2005 & 55 & 1.40 & 65 & 0.288 & 317 & 1 \\
\hline br05yp015 & Brown & 5 & 16 & 2005 & 54 & 1.34 & 64 & 0.267 & 320 & 1 \\
\hline br05yp020 & Brown & 5 & 16 & 2005 & 60 & 2.04 & 70 & 0.444 & 321 & 1 \\
\hline br05yp030 & Brown & 5 & 16 & 2005 & 48 & 1.09 & 65 & 0.222 & 322 & 1 \\
\hline br05yp026 & Brown & 5 & 16 & 2005 & 52 & 1.23 & 64 & 0.245 & 324 & 1 \\
\hline br05yp032 & Brown & 5 & 16 & 2005 & 50 & 1.04 & 65 & 0.207 & 325 & 1 \\
\hline br05yp011 & Brown & 5 & 16 & 2005 & 50 & 1.04 & 61 & 0.194 & 326 & 1 \\
\hline br05yp033 & Brown & 5 & 16 & 2005 & 48 & 0.92 & 67 & 0.185 & 336 & 1 \\
\hline br05yp024 & Brown & 5 & 16 & 2005 & 52 & 1.12 & 66 & 0.215 & 345 & 1 \\
\hline br05yp040 & Brown & 5 & 16 & 2005 & 54 & 1.45 & 67 & 0.279 & 351 & 1 \\
\hline br05yp019 & Brown & 5 & 16 & 2005 & 55 & 1.28 & 74 & 0.267 & 356 & 1 \\
\hline br05yp014 & Brown & 5 & 16 & 2005 & 59 & 1.64 & 73 & 0.330 & 362 & 1 \\
\hline br05yp034 & Brown & 5 & 16 & 2005 & 48 & 1.00 & 67 & 0.184 & 363 & 1 \\
\hline br05yp005 & Brown & 5 & 16 & 2005 & 49 & 0.97 & 78 & 0.207 & 367 & 1 \\
\hline br05yp021 & Brown & 5 & 16 & 2005 & 58 & 1.69 & 77 & 0.354 & 370 & 1 \\
\hline br05yp036 & Brown & 5 & 16 & 2005 & 57 & 1.67 & 73 & 0.328 & 373 & 1 \\
\hline br05yp027 & Brown & 5 & 16 & 2005 & 58 & 1.66 & 76 & 0.338 & 373 & 1 \\
\hline br05yp022 & Brown & 5 & 16 & 2005 & 53 & 1.24 & 74 & 0.245 & 375 & 1 \\
\hline br05yp004 & Brown & 5 & 16 & 2005 & 49 & 1.02 & 79 & 0.212 & 379 & 1 \\
\hline br05yp013 & Brown & 5 & 16 & 2005 & 57 & 1.55 & 81 & 0.330 & 382 & 1 \\
\hline br05yp012 & Brown & 5 & 16 & 2005 & 56 & 1.45 & 84 & 0.316 & 388 & 1 \\
\hline br05yp029 & Brown & 5 & 16 & 2005 & 48 & 0.93 & 78 & 0.186 & 392 & 1 \\
\hline br05yp001 & Brown & 5 & 16 & 2005 & 60 & 1.83 & 74 & 0.342 & 394 & 1 \\
\hline br05yp037 & Brown & 5 & 16 & 2005 & 52 & 1.40 & 80 & 0.284 & 395 & 1 \\
\hline br05yp017 & Brown & 5 & 16 & 2005 & 57 & 1.58 & 91 & 0.353 & 409 & 1 \\
\hline br05yp039 & Brown & 5 & 16 & 2005 & 52 & 1.30 & 84 & 0.264 & 415 & 1 \\
\hline br05yp038 & Brown & 5 & 16 & 2005 & 49 & 0.97 & 83 & 0.189 & 427 & 1 \\
\hline br05yp002 & Brown & 5 & 16 & 2005 & 51 & 1.22 & 82 & 0.233 & 432 & 1 \\
\hline br05yp023 & Brown & 5 & 16 & 2005 & 48 & 0.94 & 88 & 0.179 & 464 & 1 \\
\hline br05yp028 & Brown & 5 & 16 & 2005 & 59 & 1.75 & 93 & 0.338 & 480 & 1 \\
\hline br05yp025 & Brown & 5 & 16 & 2005 & 56 & 1.47 & 105 & 0.315 & 491 & 1 \\
\hline ре05ур004 & Peary & 5 & 15 & 2005 & 51 & 1.16 & 35 & 0.248 & 166 & \\
\hline
\end{tabular}




\begin{tabular}{|c|c|c|c|c|c|c|c|c|c|c|}
\hline \multirow[b]{2}{*}{ Sample ID } & \multirow[b]{2}{*}{ Lake } & \multicolumn{3}{|c|}{ Collection date } & \multirow{2}{*}{$\begin{array}{c}\text { Total length } \\
(\mathrm{mm})\end{array}$} & \multirow{2}{*}{\begin{tabular}{|c|} 
Fish \\
wet weight \\
(g)
\end{tabular}} & $\begin{array}{c}\text { Total mercury } \\
\text { wet weight }\end{array}$ & $\begin{array}{c}\text { Fish } \\
\text { dry weight }\end{array}$ & $\begin{array}{c}\text { Total mercury } \\
\text { dry weight }\end{array}$ & Age \\
\hline & & Month & Day & Year & & & & & & (years) \\
\hline ре05yp002 & Peary & 5 & 15 & 2005 & 57 & 1.61 & 37 & 0.356 & 170 & 1 \\
\hline ре05yp001 & Peary & 5 & 15 & 2005 & 55 & 1.47 & 36 & 0.312 & 170 & 1 \\
\hline ре05ур007 & Peary & 5 & 15 & 2005 & 58 & 1.65 & 42 & 0.364 & 191 & 1 \\
\hline pe05yp003 & Peary & 5 & 15 & 2005 & 52 & 1.14 & 44 & 0.240 & 210 & 1 \\
\hline pe05yp005 & Peary & 5 & 15 & 2005 & 57 & 1.48 & 46 & 0.313 & 217 & 1 \\
\hline ре05yp006 & Peary & 5 & 15 & 2005 & 52 & 1.08 & 46 & 0.227 & 220 & 1 \\
\hline ре05ур009 & Peary & 5 & 15 & 2005 & 54 & 1.26 & 127 & 0.266 & 599 & 1 \\
\hline ре05yp011 & Peary & 5 & 15 & 2005 & 53 & 1.15 & 120 & 0.228 & 606 & 1 \\
\hline ре05yp013 & Peary & 5 & 15 & 2005 & 58 & 1.58 & 132 & 0.326 & 642 & 1 \\
\hline ре05yp010 & Peary & 5 & 15 & 2005 & 56 & 1.48 & 140 & 0.317 & 654 & 1 \\
\hline ре05yp014 & Peary & 5 & 15 & 2005 & 55 & 1.29 & 150 & 0.256 & 753 & 1 \\
\hline ре05yp008 & Peary & 5 & 15 & 2005 & 56 & 1.37 & 168 & 0.296 & 775 & 1 \\
\hline ре05yp012 & Peary & 5 & 15 & 2005 & 54 & 1.22 & 162 & 0.246 & 803 & 1 \\
\hline ry05yp008 & Ryan & 5 & 16 & 2005 & 52 & 1.19 & 35 & 0.258 & 160 & 1 \\
\hline ry05yp009 & Ryan & 5 & 16 & 2005 & 51 & 1.14 & 36 & 0.232 & 175 & 1 \\
\hline ry05yp013 & Ryan & 5 & 16 & 2005 & 48 & 0.92 & 37 & 0.182 & 185 & 1 \\
\hline ry05yp010 & Ryan & 5 & 16 & 2005 & 50 & 0.99 & 37 & 0.188 & 197 & 1 \\
\hline ry05yp012 & Ryan & 5 & 16 & 2005 & 55 & 1.40 & 47 & 0.300 & 219 & 1 \\
\hline ry05yp014 & Ryan & 5 & 16 & 2005 & 43 & 0.73 & 42 & 0.134 & 229 & 1 \\
\hline ry05yp011 & Ryan & 5 & 16 & 2005 & 47 & 0.91 & 48 & 0.190 & 230 & 1 \\
\hline ry05yp001 & Ryan & 5 & 15 & 2005 & 64 & 2.06 & 96 & 0.441 & 446 & 1 \\
\hline ry05yp003 & Ryan & 5 & 16 & 2005 & 51 & 1.09 & 144 & 0.238 & 662 & 1 \\
\hline ry05yp002 & Ryan & 5 & 15 & 2005 & 43 & 0.77 & 112 & 0.127 & 678 & 1 \\
\hline ry05yp007 & Ryan & 5 & 16 & 2005 & 47 & 1.08 & 127 & 0.193 & 712 & 1 \\
\hline ry05yp005 & Ryan & 5 & 16 & 2005 & 49 & 0.95 & 150 & 0.198 & 720 & 1 \\
\hline ry05yp006 & Ryan & 5 & 16 & 2005 & 52 & 1.18 & 157 & 0.253 & 730 & 1 \\
\hline ry05yp004 & Ryan & 5 & 16 & 2005 & 48 & 0.89 & 174 & 0.185 & 836 & 1 \\
\hline sh05yp010 & Shoepack & 5 & 13 & 2005 & 60 & 1.88 & 50 & 0.350 & 269 & 1 \\
\hline sh05yp029 & Shoepack & 5 & 13 & 2005 & 55 & 1.30 & 50 & 0.239 & 269 & 1 \\
\hline sh05yp006 & Shoepack & 5 & 13 & 2005 & 55 & 1.24 & 49 & 0.196 & 308 & 1 \\
\hline sh05yp019 & Shoepack & 5 & 13 & 2005 & 57 & 1.43 & 47 & 0.218 & 311 & 1 \\
\hline sh05yp004 & Shoepack & 5 & 13 & 2005 & 66 & 2.49 & 60 & 0.458 & 325 & 2 \\
\hline sh05yp013 & Shoepack & 5 & 13 & 2005 & 57 & 1.45 & 65 & 0.246 & 382 & 1 \\
\hline sh05yp022 & Shoepack & 5 & 13 & 2005 & 62 & 1.87 & 69 & 0.326 & 399 & 1 \\
\hline sh05yp002 & Shoepack & 5 & 13 & 2005 & 75 & 3.34 & 84 & 0.631 & 442 & 2 \\
\hline sh05yp025 & Shoepack & 5 & 13 & 2005 & 61 & 2.09 & 88 & 0.375 & 492 & 1 \\
\hline sh05yp030 & Shoepack & 5 & 13 & 2005 & 59 & 1.69 & 95 & 0.279 & 573 & 1 \\
\hline sh05yp008 & Shoepack & 5 & 13 & 2005 & 61 & 2.03 & 112 & 0.371 & 614 & 1 \\
\hline sh05yp007 & Shoepack & 5 & 13 & 2005 & 59 & 1.31 & 97 & 0.205 & 617 & 1 \\
\hline sh05yp012 & Shoepack & 5 & 13 & 2005 & 57 & 1.53 & 111 & 0.268 & 631 & 1 \\
\hline sh05yp023 & Shoepack & 5 & 13 & 2005 & 58 & 1.46 & 103 & 0.237 & 636 & 1 \\
\hline sh05yp011 & Shoepack & 5 & 13 & 2005 & 62 & 1.73 & 113 & 0.308 & 637 & 1 \\
\hline sh05yp021 & Shoepack & 5 & 13 & 2005 & 61 & 1.73 & 116 & 0.313 & 641 & 1 \\
\hline sh05yp032 & Shoepack & 5 & 13 & 2005 & 56 & 1.16 & 121 & 0.218 & 642 & 1 \\
\hline sh05yp015 & Shoepack & 5 & 13 & 2005 & 65 & 2.00 & 116 & 0.338 & 685 & 2 \\
\hline sh05yp003 & Shoepack & 5 & 13 & 2005 & 62 & 1.89 & 139 & 0.359 & 729 & 1 \\
\hline sh05yp001 & Shoepack & 5 & 13 & 2005 & 64 & 1.96 & 135 & 0.362 & 730 & 1 \\
\hline sh05yp024 & Shoepack & 5 & 13 & 2005 & 61 & 1.80 & 121 & 0.290 & 748 & 1 \\
\hline sh05yp005 & Shoepack & 5 & 13 & 2005 & 62 & 1.69 & 130 & 0.288 & 760 & 1 \\
\hline sh05yp027 & Shoepack & 5 & 13 & 2005 & 60 & 1.74 & 135 & 0.303 & 774 & 1 \\
\hline sh05yp016 & Shoepack & 5 & 13 & 2005 & 60 & 1.64 & 132 & 0.280 & 775 & 1 \\
\hline sh05yp031 & Shoepack & 5 & 13 & 2005 & 64 & 2.04 & 140 & 0.358 & 801 & 1 \\
\hline sh05yp018 & Shoepack & 5 & 13 & 2005 & 63 & 2.00 & 152 & 0.357 & 853 & 1 \\
\hline sh05yp017 & Shoepack & 5 & 13 & 2005 & 60 & 1.71 & 150 & 0.288 & 891 & 1 \\
\hline sh05yp026 & Shoepack & 5 & 13 & 2005 & 63 & 1.98 & 151 & 0.335 & 893 & 1 \\
\hline sh05yp009 & Shoepack & 5 & 13 & 2005 & 63 & 1.79 & 149 & 0.294 & 904 & 1 \\
\hline sh05yp014 & Shoepack & 5 & 13 & 2005 & 60 & 1.22 & 145 & 0.193 & 918 & 1 \\
\hline sh05yp020 & Shoepack & 5 & 13 & 2005 & 61 & 1.69 & 159 & 0.283 & 950 & 1 \\
\hline br06yp017 & Brown & 5 & 15 & 2006 & 53 & 1.38 & 98 & 0.294 & 460 & 1 \\
\hline br06yp013 & Brown & 5 & 15 & 2006 & 54 & 1.32 & 103 & 0.249 & 544 & 1 \\
\hline br06yp018 & Brown & 5 & 15 & 2006 & 50 & 1.08 & 119 & 0.235 & 549 & 1 \\
\hline br06yp003 & Brown & 5 & 15 & 2006 & 60 & 1.88 & 124 & 0.413 & 564 & 1 \\
\hline br06yp020 & Brown & 5 & 15 & 2006 & 51 & 1.11 & 123 & 0.237 & 576 & 1 \\
\hline br06yp016 & Brown & 5 & 15 & 2006 & 53 & 1.24 & 123 & 0.260 & 589 & 1 \\
\hline br06yp012 & Brown & 5 & 15 & 2006 & 54 & 1.33 & 118 & 0.261 & 600 & 1 \\
\hline br06yp008 & Brown & 5 & 15 & 2006 & 53 & 1.20 & 132 & 0.260 & 609 & 1 \\
\hline br06yp014 & Brown & 5 & 15 & 2006 & 52 & 1.20 & 129 & 0.251 & 616 & 1 \\
\hline br06yp019 & Brown & 5 & 15 & 2006 & 49 & 1.05 & 136 & 0.230 & 619 & \\
\hline br06yp010 & Brown & 5 & 15 & 2006 & 53 & 1.17 & 132 & 0.249 & 621 & 1 \\
\hline br06yp007 & Brown & 5 & 15 & 2006 & 50 & 1.12 & 123 & 0.222 & 623 & 1 \\
\hline br06yp001 & Brown & 5 & 15 & 2006 & 54 & 1.41 & 133 & 0.297 & 633 & 1 \\
\hline br06yp009 & Brown & 5 & 15 & 2006 & 54 & 1.30 & 122 & 0.245 & 648 & 1 \\
\hline br06yp006 & Brown & 5 & 15 & 2006 & 55 & 1.32 & 134 & 0.268 & 660 & \\
\hline br06yp026 & Brown & 5 & 15 & 2006 & 51 & 1.09 & 144 & 0.236 & 663 & \\
\hline br06yp021 & Brown & 5 & 15 & 2006 & 51 & 1.05 & 137 & 0.215 & 668 & \\
\hline br06yp024 & Brown & 5 & 15 & 2006 & 55 & 1.30 & 149 & 0.289 & 670 & \\
\hline
\end{tabular}




\begin{tabular}{|c|c|c|c|c|c|c|c|c|c|c|}
\hline \multirow[b]{2}{*}{ Sample ID } & \multirow[b]{2}{*}{ Lake } & \multicolumn{3}{|c|}{ Collection date } & \multirow{2}{*}{$\begin{array}{l}\text { Total length } \\
(\mathrm{mm})\end{array}$} & \multirow{2}{*}{$\begin{array}{c}\text { Fish } \\
\text { wet weight } \\
\text { (g) }\end{array}$} & $\begin{array}{c}\text { Total mercury } \\
\text { wet weight }\end{array}$ & $\begin{array}{c}\text { Fish } \\
\text { dry weight }\end{array}$ & $\begin{array}{l}\text { Total mercury } \\
\text { dry weight }\end{array}$ & Age \\
\hline & & Month & Day & Year & & & & & & (years) \\
\hline br06yp027 & Brown & 5 & 15 & 2006 & 58 & 1.56 & 148 & 0.345 & 671 & 1 \\
\hline br06yp011 & Brown & 5 & 15 & 2006 & 57 & 1.56 & 134 & 0.309 & 674 & 1 \\
\hline br06yp030 & Brown & 5 & 15 & 2006 & 51 & 1.07 & 149 & 0.231 & 691 & 1 \\
\hline br06yp004 & Brown & 5 & 15 & 2006 & 51 & 1.05 & 135 & 0.205 & 693 & 1 \\
\hline br06yp015 & Brown & 5 & 15 & 2006 & 53 & 1.22 & 142 & 0.248 & 699 & 1 \\
\hline br06yp022 & Brown & 5 & 15 & 2006 & 52 & 1.13 & 150 & 0.240 & 707 & 1 \\
\hline br06yp025 & Brown & 5 & 15 & 2006 & 56 & 1.35 & 158 & 0.290 & 737 & 1 \\
\hline br06yp029 & Brown & 5 & 15 & 2006 & 55 & 1.19 & 165 & 0.253 & 776 & 1 \\
\hline br06yp023 & Brown & 5 & 15 & 2006 & 51 & 1.15 & 164 & 0.240 & 786 & 1 \\
\hline br06yp002 & Brown & 5 & 15 & 2006 & 57 & 1.42 & 158 & 0.285 & 787 & 1 \\
\hline br06yp005 & Brown & 5 & 15 & 2006 & 51 & 1.20 & 172 & 0.254 & 811 & 1 \\
\hline br06yp028 & Brown & 5 & 15 & 2006 & 50 & 0.91 & 175 & 0.196 & 812 & 1 \\
\hline ре06yp017 & Peary & 5 & 15 & 2006 & 53 & 1.38 & 101 & 0.304 & 460 & 1 \\
\hline ре06yp013 & Peary & 5 & 15 & 2006 & 59 & 1.72 & 115 & 0.362 & 544 & 1 \\
\hline рe06yp018 & Peary & 5 & 15 & 2006 & 57 & 1.62 & 120 & 0.354 & 549 & 1 \\
\hline ре06yр003 & Peary & 5 & 15 & 2006 & 73 & 3.31 & 113 & 0.665 & 564 & 1 \\
\hline ре06yp020 & Peary & 5 & 15 & 2006 & 55 & 1.65 & 126 & 0.361 & 576 & 1 \\
\hline ре06ур016 & Peary & 5 & 15 & 2006 & 57 & 0.99 & 105 & 0.177 & 589 & 1 \\
\hline ре06yp012 & Peary & 5 & 15 & 2006 & 57 & 1.65 & 128 & 0.353 & 600 & 1 \\
\hline ре06yр008 & Peary & 5 & 15 & 2006 & 56 & 1.71 & 139 & 0.391 & 609 & 1 \\
\hline ре06yp014 & Peary & 5 & 15 & 2006 & 64 & 2.28 & 130 & 0.481 & 616 & 1 \\
\hline ре06yp019 & Peary & 5 & 15 & 2006 & 52 & 1.37 & 121 & 0.267 & 619 & 1 \\
\hline ре06yp010 & Peary & 5 & 15 & 2006 & 53 & 1.29 & 141 & 0.292 & 621 & 1 \\
\hline ре06yp007 & Peary & 5 & 15 & 2006 & 51 & 1.19 & 142 & 0.271 & 623 & 1 \\
\hline ре06yр001 & Peary & 5 & 15 & 2006 & 60 & 1.80 & 132 & 0.375 & 633 & 1 \\
\hline ре06yp009 & Peary & 5 & 15 & 2006 & 60 & 1.86 & 128 & 0.368 & 648 & 1 \\
\hline ре06yр006 & Peary & 5 & 15 & 2006 & 51 & 1.05 & 116 & 0.184 & 660 & 1 \\
\hline ре06yp026 & Peary & 5 & 15 & 2006 & 54 & 1.36 & 120 & 0.245 & 663 & 1 \\
\hline ре06ур021 & Peary & 5 & 15 & 2006 & 58 & 1.78 & 148 & 0.394 & 668 & 1 \\
\hline ре06ур024 & Peary & 5 & 15 & 2006 & 58 & 1.80 & 142 & 0.381 & 670 & 1 \\
\hline ре06yp027 & Peary & 5 & 15 & 2006 & 55 & 1.51 & 143 & 0.322 & 671 & 1 \\
\hline ре06yp011 & Peary & 5 & 15 & 2006 & 55 & 1.52 & 149 & 0.337 & 674 & 1 \\
\hline ре06yp030 & Peary & 5 & 15 & 2006 & 58 & 1.90 & 151 & 0.414 & 691 & 1 \\
\hline ре06ур004 & Peary & 5 & 15 & 2006 & 63 & 2.49 & 136 & 0.488 & 693 & 1 \\
\hline ре06yp015 & Peary & 5 & 15 & 2006 & 64 & 2.18 & 142 & 0.443 & 699 & 1 \\
\hline ре06ур022 & Peary & 5 & 15 & 2006 & 59 & 1.81 & 156 & 0.398 & 707 & 1 \\
\hline ре06yp025 & Peary & 5 & 15 & 2006 & 60 & 1.97 & 157 & 0.420 & 737 & 1 \\
\hline ре06ур029 & Peary & 5 & 15 & 2006 & 50 & 1.29 & 159 & 0.264 & 776 & 1 \\
\hline ре06yp023 & Peary & 5 & 15 & 2006 & 62 & 2.44 & 162 & 0.504 & 786 & 1 \\
\hline ре06yp002 & Peary & 5 & 15 & 2006 & 59 & 1.97 & 161 & 0.404 & 787 & 1 \\
\hline ре06yp005 & Peary & 5 & 15 & 2006 & 55 & 1.48 & 177 & 0.324 & 811 & 1 \\
\hline ре06yp028 & Peary & 5 & 15 & 2006 & 49 & 1.03 & 165 & 0.209 & 812 & 1 \\
\hline ry06yp002 & Ryan & 5 & 16 & 2006 & 58 & 1.73 & 98 & 0.360 & 469 & 1 \\
\hline ry06yp016 & Ryan & 5 & 16 & 2006 & 61 & 2.33 & 107 & 0.485 & 516 & 1 \\
\hline ry06yp004 & Ryan & 5 & 16 & 2006 & 52 & 1.29 & 110 & 0.267 & 532 & 1 \\
\hline ry06yp026 & Ryan & 5 & 16 & 2006 & 57 & 1.72 & 128 & 0.380 & 580 & 1 \\
\hline ry06yp013 & Ryan & 5 & 16 & 2006 & 54 & 1.85 & 123 & 0.363 & 629 & 1 \\
\hline ry06yp003 & Ryan & 5 & 16 & 2006 & 54 & 1.46 & 138 & 0.307 & 657 & 1 \\
\hline ry06yp019 & Ryan & 5 & 16 & 2006 & 53 & 1.35 & 147 & 0.294 & 676 & 1 \\
\hline ry06yp009 & Ryan & 5 & 16 & 2006 & 56 & 1.58 & 150 & 0.348 & 680 & 1 \\
\hline ry06yp015 & Ryan & 5 & 16 & 2006 & 60 & 1.89 & 156 & 0.420 & 701 & 1 \\
\hline ry06yp011 & Ryan & 5 & 16 & 2006 & 63 & 2.13 & 153 & 0.455 & 718 & 1 \\
\hline ry06yp005 & Ryan & 5 & 16 & 2006 & 53 & 1.45 & 155 & 0.307 & 733 & 1 \\
\hline ry06yp022 & Ryan & 5 & 16 & 2006 & 59 & 2.12 & 156 & 0.440 & 751 & 1 \\
\hline ry06yp025 & Ryan & 5 & 16 & 2006 & 56 & 1.52 & 158 & 0.320 & 751 & 1 \\
\hline ry06yp001 & Ryan & 5 & 16 & 2006 & 59 & 2.13 & 159 & 0.448 & 757 & 1 \\
\hline ry06yp006 & Ryan & 5 & 16 & 2006 & 56 & 1.74 & 150 & 0.342 & 764 & 1 \\
\hline ry06yp017 & Ryan & 5 & 16 & 2006 & 54 & 1.51 & 152 & 0.299 & 769 & 1 \\
\hline ry06yp020 & Ryan & 5 & 16 & 2006 & 52 & 1.48 & 171 & 0.324 & 780 & 1 \\
\hline ry06yp027 & Ryan & 5 & 16 & 2006 & 56 & 1.47 & 171 & 0.313 & 805 & 1 \\
\hline ry06yp018 & Ryan & 5 & 16 & 2006 & 51 & 1.25 & 168 & 0.259 & 813 & 1 \\
\hline ry06yp024 & Ryan & 5 & 16 & 2006 & 58 & 1.89 & 180 & 0.408 & 834 & 1 \\
\hline ry06yp028 & Ryan & 5 & 16 & 2006 & 56 & 1.70 & 172 & 0.348 & 839 & 1 \\
\hline ry06yp014 & Ryan & 5 & 16 & 2006 & 59 & 1.89 & 181 & 0.405 & 843 & 1 \\
\hline ry06yp008 & Ryan & 5 & 16 & 2006 & 56 & 1.83 & 166 & 0.360 & 846 & 1 \\
\hline ry06yp012 & Ryan & 5 & 16 & 2006 & 59 & 1.94 & 184 & 0.420 & 851 & 1 \\
\hline ry06yp029 & Ryan & 5 & 16 & 2006 & 54 & 1.62 & 180 & 0.338 & 860 & 1 \\
\hline ry06yp021 & Ryan & 5 & 16 & 2006 & 55 & 1.50 & 186 & 0.320 & 871 & 1 \\
\hline ry06yp030 & Ryan & 5 & 16 & 2006 & 56 & 1.79 & 183 & 0.368 & 891 & 1 \\
\hline ry06yp007 & Ryan & 5 & 16 & 2006 & 63 & 2.07 & 204 & 0.439 & 962 & 1 \\
\hline ry06yp023 & Ryan & 5 & 16 & 2006 & 59 & 1.66 & 186 & 0.310 & 994 & 1 \\
\hline ry06yp010 & Ryan & 5 & 16 & 2006 & 56 & 1.72 & 221 & 0.318 & 1,196 & 1 \\
\hline sh06yp037 & Shoepack & 5 & 17 & 2006 & 85 & 6.33 & 59 & 1.367 & 275 & 3 \\
\hline sh06yp033 & Shoepack & 5 & 17 & 2006 & 89 & 6.26 & 79 & 1.418 & 347 & 3 \\
\hline sh06yp035 & Shoepack & 5 & 17 & 2006 & 87 & 6.29 & 82 & 1.366 & 378 & 3 \\
\hline sh06yp020 & Shoepack & 5 & 17 & 2006 & 71 & 3.10 & 82 & 0.642 & 396 & \\
\hline
\end{tabular}




\begin{tabular}{|c|c|c|c|c|c|c|c|c|c|c|}
\hline \multirow[b]{2}{*}{ Sample ID } & \multirow[b]{2}{*}{ Lake } & \multicolumn{3}{|c|}{ Collection date } & \multirow{2}{*}{$\begin{array}{c}\text { Total length } \\
(\mathrm{mm})\end{array}$} & \multirow{2}{*}{$\begin{array}{c}\text { Fish } \\
\text { wet weight } \\
(\mathrm{g})\end{array}$} & \multirow{2}{*}{$\begin{array}{c}\text { Total mercury } \\
\text { wet weight } \\
\text { (ng/g) }\end{array}$} & \multirow{2}{*}{$\begin{array}{c}\text { Fish } \\
\text { dry weight } \\
\text { (g) }\end{array}$} & \multirow{2}{*}{$\begin{array}{l}\text { Total mercury } \\
\text { dry weight } \\
\text { (ng/g) }\end{array}$} & \multirow{2}{*}{$\begin{array}{c}\text { Age } \\
\text { (years) }\end{array}$} \\
\hline & & Month & Day & Year & & & & & & \\
\hline sh06yp055 & Shoepack & 5 & 17 & 2006 & 67 & 2.36 & 89 & 0.457 & 459 & 2 \\
\hline sh06yp013 & Shoepack & 5 & 17 & 2006 & 71 & 3.33 & 95 & 0.645 & 488 & 2 \\
\hline sh06yp010 & Shoepack & 5 & 17 & 2006 & 70 & 2.83 & 101 & 0.575 & 498 & 1 \\
\hline sh06yp043 & Shoepack & 5 & 17 & 2006 & 89 & 6.45 & 108 & 1.397 & 499 & 3 \\
\hline sh06yp047 & Shoepack & 5 & 17 & 2006 & 83 & 4.52 & 103 & 0.909 & 511 & 3 \\
\hline sh06yp016 & Shoepack & 5 & 17 & 2006 & 71 & 3.53 & 112 & 0.767 & 516 & 2 \\
\hline sh06yp012 & Shoepack & 5 & 17 & 2006 & 65 & 2.02 & 97 & 0.378 & 519 & 1 \\
\hline sh06yp008 & Shoepack & 5 & 17 & 2006 & 75 & 3.91 & 104 & 0.758 & 536 & 2 \\
\hline sh06yp060 & Shoepack & 5 & 17 & 2006 & 76 & 3.87 & 116 & 0.816 & 550 & 2 \\
\hline sh06yp024 & Shoepack & 5 & 17 & 2006 & 70 & 2.88 & 104 & 0.540 & 554 & 2 \\
\hline sh06yp059 & Shoepack & 5 & 17 & 2006 & 74 & 3.45 & 131 & 0.753 & 601 & 2 \\
\hline sh06yp058 & Shoepack & 5 & 17 & 2006 & 75 & 3.74 & 133 & 0.812 & 614 & scales missing \\
\hline sh06yp040 & Shoepack & 5 & 17 & 2006 & 83 & 5.04 & 132 & 1.078 & 617 & 3 \\
\hline sh06yp011 & Shoepack & 5 & 17 & 2006 & 73 & 3.44 & 125 & 0.692 & 624 & 2 \\
\hline sh06yp004 & Shoepack & 5 & 17 & 2006 & 68 & 2.39 & 128 & 0.482 & 633 & 1 \\
\hline sh06yp017 & Shoepack & 5 & 17 & 2006 & 67 & 2.13 & 119 & 0.400 & 633 & 1 \\
\hline sh06yp030 & Shoepack & 5 & 17 & 2006 & 66 & 2.47 & 118 & 0.461 & 635 & 1 \\
\hline sh06yp025 & Shoepack & 5 & 17 & 2006 & 74 & 3.49 & 131 & 0.717 & 639 & 2 \\
\hline sh06yp056 & Shoepack & 5 & 17 & 2006 & 71 & 3.32 & 135 & 0.698 & 643 & 2 \\
\hline sh06yp052 & Shoepack & 5 & 17 & 2006 & 80 & 4.50 & 142 & 0.994 & 645 & 3 \\
\hline sh06yp029 & Shoepack & 5 & 17 & 2006 & 70 & 2.73 & 124 & 0.522 & 648 & 1 \\
\hline sh06yp021 & Shoepack & 5 & 17 & 2006 & 67 & 2.64 & 133 & 0.532 & 660 & 1 \\
\hline sh06yp032 & Shoepack & 5 & 17 & 2006 & 84 & 4.61 & 130 & 0.902 & 662 & 3 \\
\hline sh06yp002 & Shoepack & 5 & 17 & 2006 & 70 & 2.88 & 132 & 0.561 & 675 & 2 \\
\hline sh06yp019 & Shoepack & 5 & 17 & 2006 & 75 & 3.65 & 136 & 0.731 & 678 & 2 \\
\hline sh06yp027 & Shoepack & 5 & 17 & 2006 & 68 & 2.66 & 135 & 0.530 & 679 & 1 \\
\hline sh06yp001 & Shoepack & 5 & 17 & 2006 & 73 & 2.86 & 143 & 0.572 & 713 & 2 \\
\hline sh06yp057 & Shoepack & 5 & 17 & 2006 & 75 & 3.46 & 136 & 0.651 & 722 & 3 \\
\hline sh06yp038 & Shoepack & 5 & 17 & 2006 & 84 & 5.59 & 150 & 1.144 & 733 & 3 \\
\hline sh06yp006 & Shoepack & 5 & 17 & 2006 & 72 & 2.79 & 125 & 0.468 & 743 & 2 \\
\hline sh06yp007 & Shoepack & 5 & 17 & 2006 & 60 & 1.52 & 126 & 0.255 & 754 & 1 \\
\hline sh06yp009 & Shoepack & 5 & 17 & 2006 & 65 & 2.05 & 142 & 0.384 & 756 & 1 \\
\hline sh06yp054 & Shoepack & 5 & 17 & 2006 & 81 & 4.25 & 154 & 0.860 & 762 & 3 \\
\hline sh06yp018 & Shoepack & 5 & 17 & 2006 & 70 & 2.92 & 145 & 0.544 & 776 & 2 \\
\hline sh06yp023 & Shoepack & 5 & 17 & 2006 & 72 & 3.30 & 160 & 0.672 & 784 & 2 \\
\hline sh06yp005 & Shoepack & 5 & 17 & 2006 & 73 & 3.30 & 151 & 0.634 & 787 & 2 \\
\hline sh06yp049 & Shoepack & 5 & 17 & 2006 & 88 & 6.14 & 174 & 1.305 & 820 & 3 \\
\hline sh06yp003 & Shoepack & 5 & 17 & 2006 & 70 & 2.97 & 169 & 0.588 & 851 & 1 \\
\hline sh06yp026 & Shoepack & 5 & 17 & 2006 & 78 & 3.67 & 173 & 0.744 & 854 & 2 \\
\hline sh06yp041 & Shoepack & 5 & 17 & 2006 & 83 & 4.98 & 183 & 1.048 & 868 & 3 \\
\hline sh06yp036 & Shoepack & 5 & 17 & 2006 & 80 & 4.02 & 170 & 0.780 & 876 & 3 \\
\hline sh06yp028 & Shoepack & 5 & 17 & 2006 & 75 & 3.38 & 171 & 0.653 & 887 & 2 \\
\hline sh06yp014 & Shoepack & 5 & 17 & 2006 & 74 & 3.12 & 164 & 0.574 & 893 & 2 \\
\hline sh06yp053 & Shoepack & 5 & 17 & 2006 & 81 & 4.27 & 192 & 0.863 & 950 & 3 \\
\hline sh06yp022 & Shoepack & 5 & 17 & 2006 & 70 & 2.84 & 179 & 0.523 & 971 & 2 \\
\hline sh06yp051 & Shoepack & 5 & 17 & 2006 & 80 & 3.93 & 181 & 0.733 & 971 & 3 \\
\hline sh06yp031 & Shoepack & 5 & 17 & 2006 & 83 & 4.64 & 204 & 0.936 & 1,013 & 3 \\
\hline sh06yp039 & Shoepack & 5 & 17 & 2006 & 90 & 6.38 & 196 & 1.220 & 1,027 & 4 \\
\hline sh06yp045 & Shoepack & 5 & 17 & 2006 & 89 & 5.67 & 205 & 1.109 & 1,048 & 4 \\
\hline sh06yp046 & Shoepack & 5 & 17 & 2006 & 84 & 4.78 & 214 & 0.937 & 1,090 & 3 \\
\hline sh06yp042 & Shoepack & 5 & 17 & 2006 & 80 & 4.50 & 235 & 0.927 & 1,138 & 2 \\
\hline sh06yp034 & Shoepack & 5 & 17 & 2006 & 88 & 5.27 & 232 & 0.988 & 1,238 & 3 \\
\hline sh06yp050 & Shoepack & 5 & 17 & 2006 & 82 & 3.64 & 238 & 0.650 & 1,331 & 3 \\
\hline sh06yp048 & Shoepack & 5 & 17 & 2006 & 85 & 4.29 & 280 & 0.806 & 1,490 & 3 \\
\hline
\end{tabular}


